\title{
Yang Liu
}

\section{Adolescents' Alcohol Use and Smoking in Beijing, China}

Implementing the HBSC Study and Comparing Socioeconomic Inequalities with Finland

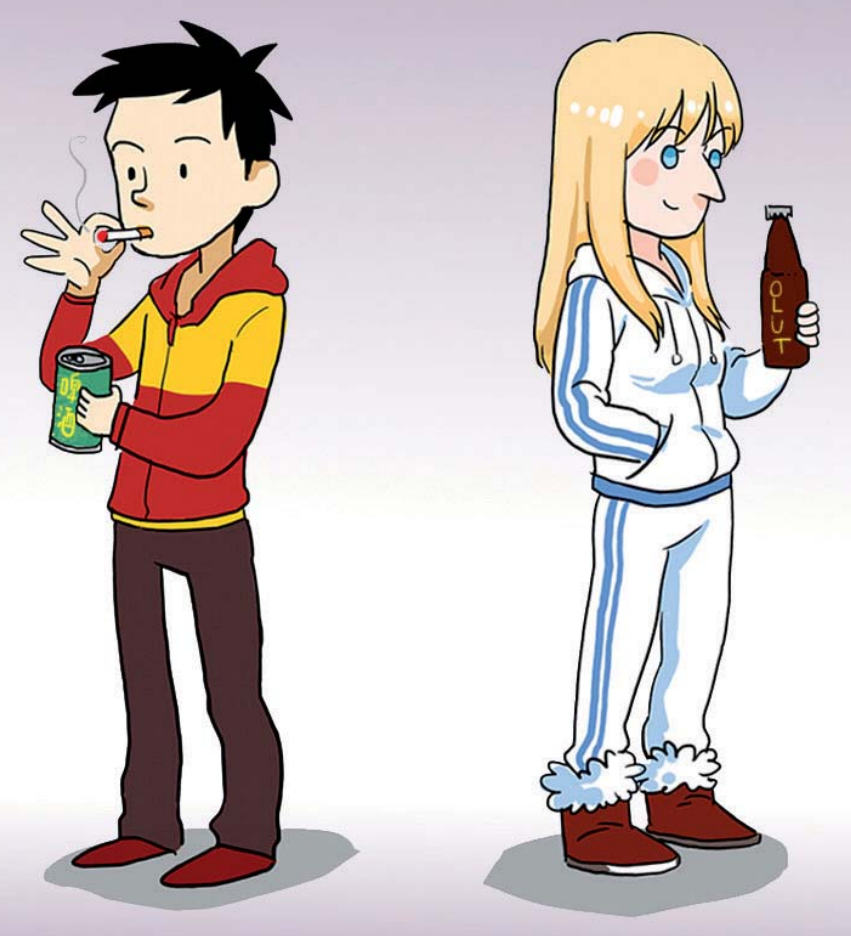




\section{Yang Liu}

\section{Adolescents' Alcohol Use and Smoking in Beijing, China}

\section{Implementing the HBSC Study and Comparing Socioeconomic Inequalities with Finland}
Esitetään Jyväskylän yliopiston liikuntatieteellisen tiedekunnan suostumuksella julkisesti tarkastettavaksi yliopiston Liikunnan salissa L303 joulukuun 18. päivänä 2013 kello 12.

Academic dissertation to be publicly discussed, by permission of the Faculty of Sport and Health Sciences of the University of Jyväskylä, in the Liikunta building, auditorium L303, on December 18, 2013 at 12 o'clock noon.

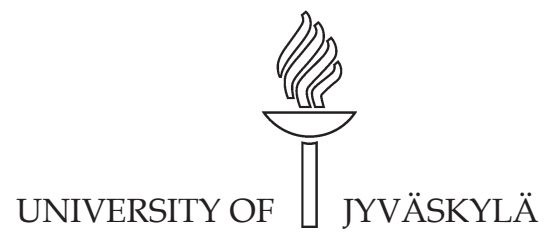

JYVÄSKYLÄ 2013 


\section{Adolescents' Alcohol Use and Smoking in Beijing, China}

Implementing the HBSC Study and Comparing Socioeconomic Inequalities with Finland 


\section{Yang Liu}

\section{Adolescents' Alcohol Use and Smoking in Beijing, China}

Implementing the HBSC Study and Comparing Socioeconomic Inequalities with Finland

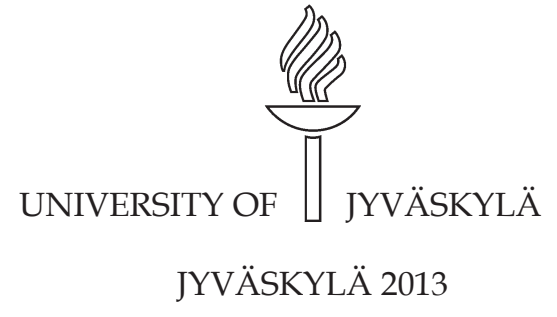


Editors

Ina Tarkka

Department of Health Sciences, University of Jyväskylä Pekka Olsbo, Sini Tuikka

Publishing Unit, University Library of Jyväskylä

Cover picture by Delan Wen and Yifei Li

URN:ISBN:978-951-39-5461-1

ISBN 978-951-39-5461-1 (PDF)

ISBN 978-951-39-5460-4 (nid.)

ISSN 0356-1070

Copyright (C 2013, by University of Jyväskylä

Jyväskylä University Printing House, Jyväskylä 2013 
If the youth are wise, society will be wise; if the youth are rich, society will be rich; if the youth are strong, society will be strong; if the youth are independent, society will be independent; if the youth are free, society will be free; if the youth progress, society will progress.

The Young China by Liang Qichao, 1900

少年智则国智; 少年富则国富; 少年强则国强; 少年独立则国独立; 少年自由则国自由; 少年进步则国进步。

《少年中国说》梁启超, 1900

To My Family 献给我的家人 


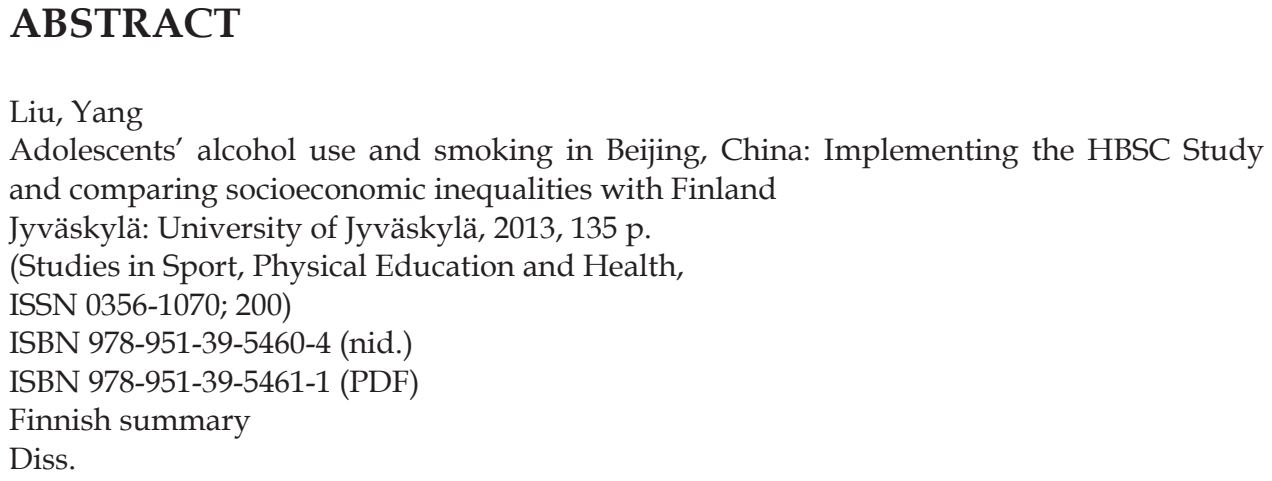

This study comprised two stages. The aim of the first stage was to examine the feasibility of implementing the Health Behaviour in School-aged Children (HBSC) survey, as well as the reliability of selected survey items in a sample of students in Beijing, China. The reliability and validity of the HBSC Family Affluence Scale (FAS), as the indicator of socioeconomic status (SES), was also investigated in a Chinese context. The aim of the second stage was to explore the associations between SES and adolescents' alcohol use and smoking in China and Finland using the HBSC survey results and to examine the similarities and differences of socioeconomic inequalities in substance use of adolescents in these two countries.

The analyses of this study are based on data from three studies: 1) test-retest study in China 2008 ( $\mathrm{n}=95)$; 2) Chinese HBSC linked project survey $2008(\mathrm{n}=5876)$; and 3) Finnish HBSC survey $2006(n=5255)$. All studies followed the HBSC study protocol. In the first stage of this study, the test-retest reliability of selected survey items related to health behaviour was assessed by the Intraclass Correlation Coefficients. Reliability and validity of the FAS were examined by the Cronbach's alpha coefficient, Spearman correlations, confirmatory factor analysis, ordinal logistic regression and the Graphic Log Linear Rasch Model. In the second stage, the sample included 2005 Chinese and 1685 Finnish 15-year-old adolescents. The associations between the FAS and alcohol use and smoking were evaluated separately in the two countries through binary logistic regression.

The results of this study showed that implementing the HBSC survey in Beijing, China is feasible, and the selected HBSC survey indicators have satisfactory test-retest reliability. Meanwhile, this study indicated that the reliability and validity of FAS, as a SES measure for adolescent, are acceptable in a Chinese context. Different patterns of socioeconomic inequalities in alcohol use and smoking between Chinese and Finnish adolescents were observed. In China, students from more affluent families were more likely to report early onset of drunkenness and daily smoking for boys, and drinking monthly and early onset of drinking for girls, while the socioeconomic inequalities in alcohol use and smoking were absent among Finnish pupils, except for girls from less affluent families being more likely to be daily smokers.

This study is the first study using the HBSC research protocol in mainland China. The findings of this study provided comprehensive information on the feasibility and reliability of the HBSC survey items in a Chinese context, which will contribute to the future Chinese HBSC study. In addition, the comparison of the socioeconomic inequalities in substance use of adolescents revealed that when planning and implementing interventions/programmes aimed at reducing young people's drinking and smoking in China, the target should be focused on those students from more affluent families, whereas in Finland, efforts should be made on the reduction of the high rates of drinking and smoking of adolescents in both genders and all socioeconomic groups.

Keywords: adolescent, alcohol use, family affluence scale, health behaviour, smoking, socioeconomic inequalities 
Yang Liu, MSc, MEd

Research Centre for Health Promotion

Department of Health Sciences

P.O. Box 35 (L)

FI-40014 University of Jyväskylä

Jyväskylä, Finland

yang.liu@jyu.fi, docliuyang@hotmail.com

Supervisors

Professor Lasse Kannas, PhD

Research Centre for Health Promotion

Department of Health Sciences

University of Jyväskylä

Jyväskylä, Finland

Lecturer Jorma Tynjälä, PhD

Research Centre for Health Promotion

Department of Health Sciences

University of Jyväskylä

Jyväskylä, Finland

Reviewers

Research Professor Sakari Karvonen, PhD

Department of Social and Health Policy and Economics

National Institute for Health and Welfare

Helsinki, Finland

Adjunct Professor Tomi Lintonen, $\mathrm{PhD}$

The Finnish Foundation for Alcohol Studies

Helsinki, Finland

Opponent

Professor Bjørn E. Holstein

National Institute of Public Health

University of Southern Denmark

Copenhagen, Denmark 


\section{ACKNOWLEDGEMENTS}

This study was carried out at the Research Centre for Health Promotion at the Department of Health Sciences in the Faculty of Sport and Health Sciences of the University of Jyväskylä, Finland, from 2008 to 2013. The present study also appertained to the international collaborative research project on comparison of schoolchildren's health behaviour between China and Finland, which initiated in 2007 by the Research Centre for Health Promotion and the China Institute of Sport Science (CISS). I was privileged to be able to work with the research teams from both countries for expanding the Health Behaviour in Schoolchildren (HBSC) study in China in which the world's most schoolchildren live. There is a multitude of people who have given a contribution to this scientific work and my doctoral study that should be acknowledged.

I wish to present my sincere gratitude towards my supervisors, Professor Lasse Kannas and Jorma Tynjälä, PhD. Six years ago, when I nearly finished my Master's study and thought future possible research field for my doctoral study, I found, by chance, the information of the HBSC study which intrigued me without knowing that actually Lasse is one of the co-founder of that study. I really appreciate Lasse for accepting me as his doctoral student and thus I had an opportunity to be involved in the later research projects. Despite the fact that he has been busy with his work, he always gives me full support for both academic and daily issues whenever I need. My profound thanks also go to Jorma, the other supervisor of mine. As the principal investigator of the Finnish HBSC research team, his professional expertise and insightful opinions helped me in overcoming all kinds of research problems related to my study. Lasse and Jorma, you have given me invaluable support and encouragement from the countless meetings and thoughtful discussions we had. This study would never have been completed without your guidance.

I would like to cordially thank Professor Tomi Lintonen and Professor Sakari Karvonen for their kind reviewing my thesis. Their inspiring criticism and profound knowledge provided valuable suggestions and comments on improving the quality of this thesis. It was my honour to have them as the preliminary examiners of my doctoral thesis. I am most grateful that Tomi kindly invited me to his home and had a four-hour fruitful discussion, as well as the thoughtful meeting I had with Sakari via Skype.

I wish to extend my warmest gratitude towards the Chinese research partners. Professor Mei Wang, as the principal investigator of Chinese HBSC study, has offered enormous support for organising and conducting the field survey in Beijing. Without your continuous support and guidance, this project would never have happened. I would like also to acknowledge Professor Ye Tian, the president of CISS, and Professor Lianshi Feng, the vice president of CISS, for their support for this collaborative research project and the future Chinese HBSC linked project. Special thanks also go to Yan Lv, MEd, who has done a lot of practical work for the Chinese HBSC study. In addition, I especially wish to express my appreciation to those over ten thousand schoolchildren from China and Finland who took part in this study, as well as all researchers and teachers involved in the surveys. The study would have been impossible without your participation. Furthermore, I would like to acknowledge the Health Behaviour in School-aged Children: a World Health Organisation Collaborative Crossnational Study.

Two co-authors of this study should be complimented. Jari Villberg, MSc, the statistician of the Research Centre for Health Promotion, provided valuable statistical help for all four articles of this thesis. His professional skills and plentiful experience 
are indeed important for this study. It is my pleasure to learn advanced statistical technique from him during my doctoral study. In addition, I owe my gratitude to Professor Torbjørn Torsheim. The idea of writing the second article of this thesis was inspired by our discussion in his office in Bergen. And he has given constructive suggestions and critical advice on it.

I gratefully acknowledge Michael Ormshaw, MSc, for proofreading all my articles and this thesis summary. You did not only check my English language and grammar, but also gave many helpful suggestions and comments. Moreover, as the only two foreigners in our research centre and two-year office roommates, I really enjoy the time when we are chatting and playing squash. I wish to thank Anne Räsänen, PhLic, as well for translating the Finnish summary of this thesis.

I would like to thank Leena Paakkari, PhD, Raili Välimaa, PhD, Kristiina Ojala, $\mathrm{PhD}$, and Pirjo Koikkalainen, MA, for your generous help during my study. I wish to thank all the other colleagues from the Research Centre for Health Promotion. It was my privilege to have been worked with so many talent researchers in such a comfortable and nice environment. Besides, I want to thank Junjie Feng, PhD, Anna-Katriina Salmikangas, $\mathrm{PhD}$, and Professor Kimmo Suomi for their guidance and help during my Master's study at the Department of Sport Sciences.

I wish to express my deepest gratitude to Professor Shulin Cheng, who has always given me guidance and encouragement in academic work and practical life since I was in Jyväskylä. Her enthusiasms in scientific research and high efficient working spirit have always spurred me on to further progress. Definitely, I will also never forget those enjoyable times spent in her home when celebrating Chinese traditional festivals.

I would like to thank my dearest friends met in Finland, including Fengyu Cong, PhD, Yikun Lu, MSc, Shumei Cheng, Mingdong Li, MSc, Lin Li, MSc, Jie Han, MSc, Meng Wang, MSc, Dayu Yang, MSc, Professor Min Hu, Gang Tong, Cong Li, Paulus Konttinen, Juha Tuominen, MSc, Pirkko Luoma, PhD, and many other friends whose names are not possible to be all listed here. Moreover, I would like to thank a group of elderly Finns with whom I have played soccer every Sunday morning in the past eight years. I was so lucky to meet all of you and I indeed appreciate that we have experienced those wonderful times together.

This study was financially supported by grants from the University of Jyväskylä, Juho Vainio Foundation, and The Finnish Foundation for Alcohol Studies. I am grateful for their generosity in funding my thesis and my life in Finland during my study.

Finally, I would like to dedicate this thesis to my beloved family. I am greatly indebted to my mother, Shuyin Yang, for her endless care and great love which have always been the support of my study and work. I wish to express my heartfelt thanks to my sister, Hong Liu, my brother-in-law, Guisheng Zhao, and my niece, Liubaihe Zhao, for their solid support. Last but not least, my darling wife, Haibin Que deserves my earnest appreciation for understanding my long time absence from her due to this study. Her love and support have been and will always be the most precious treasure of my life.

To all, THANK YOU VERY MUCH! KIITOS PALJON! 十分感谢！

Jyväskylä, 1 October, 2013

Yang Liu 刘阳 


\section{FIGURES}

FIGURE 1 Frequencies of test-retest shifts on all selected items in testretest reliability study in Beijing, China 2008, sorted according to the frequencies of no resp- onse shift, descending order $(\mathrm{n}=95)$.

FIGURE 2 The generalization of the FAS II to GLLRM (numbers in boxes are partial gamma coefficients, $\gamma^{p}$, indicate a significant correlation beyond the latent trait) in Chinese HBSC linked project survey $2008(\mathrm{~N}=5876)$.

TABLES

TABLE 1 Demographic characteristics of respondents of test-retest reliability study in Beijing, China 2008.

TABLE 2 Numbers and response rate (\%) of sample schools by age group in Chinese HBSC linked project survey 2008 and Finnish HBSC survey 2006. 56

TABLE 3 Numbers and response rate (\%) of sampled students in Chinese HBSC linked project survey 2008 and Finnish HBSC survey 2006.

TABLE $4 \quad$ Valid sample size and mean age (years and decimals) with standard deviation (SD) by gender and age group in Chinese HBSC linked project survey 2008 and Finnish HBSC survey 2006.

TABLE 5 The selected items related to health behaviours and their response alternatives in test-retest reliability study in Beijing, China 2008.

TABLE 6 ICC values of selected HBSC survey items about physical activity, sedentary behaviour and sleep by gender and age in test-retest reliability study in Beijing, China 2008

TABLE 7 ICC values of selected HBSC survey items about alcohol use and smoking of adolescents aged 15 years by gender in testretest reliability study in Beijing, China 2008

TABLE 8 ICC value of FAS II items, FAS score and FAS category by gender and age in test-retest reliability study in Beijing, China 2008 .

TABLE 9 Cronbach's alpha coefficient for FAS II items by gender and age in Chinese HBSC linked project survey $2008(\mathrm{~N}=5876) \ldots .72$

TABLE 10 Spearman correlations of FAS II items in Chinese HBSC linked project survey $2008(\mathrm{~N}=5876)$. 
TABLE 11 The distribution of independent variables by FAS category and ordinal regression results in Chinese HBSC linked project survey 2008, FAS low as the referent $(\mathrm{N}=5876)$.

TABLE 12 Percentages of adolescents' alcohol use by country, gender and FAS groups in Chinese HBSC linked project survey $2008(n=2005)$ and Finnish HBSC survey 2006(n = 1685) ........ 77

TABLE 13 The associations between alcohol use and Family Affluence Scale by gender and country, odds ratios with $95 \%$ confidence intervals in Chinese HBSC linked project survey $2008(n=2005)$ and Finnish HBSC survey $2006(n=1685)$

TABLE 14 Percentages of adolescents' smoking by country, gender and FAS groups in Chinese HBSC linked project survey 2008 $(\mathrm{n}=2005)$ and Finnish HBSC survey $2006(\mathrm{n}=1685)$. 79

TABLE 15 The associations between smoking and Family Affluence Scale by gender and country, odds ratios with $95 \%$ confidence intervals in Chinese HBSC linked project survey 2008 $(n=2005)$ and Finnish HBSC survey $2006(n=1685)$. .80 


\section{LIST OF ORIGINAL PUBLICATIONS}

The thesis is based on the following original publications which will be referred to in the text by their Roman numerals I-IV:

I Liu, Y., Wang, M., Tynjälä, J., Lv, Y., Villberg, J., Zhang, Z. \& Kannas, L. 2010. Test-retest reliability of selected items of Health Behaviour in Schoolaged Children (HBSC) survey questionnaire in Beijing, China. BMC Medical Research Methodology 10, 73.

II Liu, Y., Wang, M., Villberg, J., Torsheim, T., Tynjälä, J., Lv, Y. \& Kannas, L. 2012. Reliability and validity of family affluence scale (FAS II) among adolescents in Beijing, China. Child Indicators Research 5 (2), 235-251.

III Liu, Y., Wang, M., Tynjälä, J., Villberg, J., Lv, Y. \& Kannas, L. 2013. Socioeconomic inequalities in alcohol use of adolescents: the differences between China and Finland. International Journal of Public Health 58 (2), 177-185.

IV Liu, Y., Wang, M., Tynjälä, J., Villberg, J., Lv, Y. \& Kannas, L. 2013. Socioeconomic differences in smoking of adolescents: a comparison between China and Finland. (submitted for publication). 


\title{
LIST OF ABBREVIATIONS
}

\author{
AHLS Adolescent Health and Lifestyle Survey \\ CABRFS China Adolescent Behavioural Risk Factor Survey \\ CDC U.S. Centres for Disease Control and Prevention \\ CFA Confirmatory Factor Analysis \\ CFI Comparative Fit Index \\ CI Confidence Interval \\ CISS China Institute of Sport Science \\ CSCS China Seven Cities Study \\ DIF Differential Item Function \\ EMCDDA European Monitoring Centre for Drugs and Drug Addiction \\ ERP Event-Related Potential \\ ESPAD European School Survey Project on Alcohol and Other Drugs \\ FAS Family Affluence Scale \\ FAS II Family Affluence Scale Version II \\ FCTC Framework Convention on Tobacco Control \\ FNBE Finnish National Board of Education \\ GLLRM Graphical Log Linear Rasch Model \\ GSHS Global School Health Survey \\ GYTS Global Youth Tobacco Survey \\ HBSC Health Behaviour in School-aged Children, a WHO collaborative \\ cross-national study \\ ICC Intraclass Correlation Coefficients \\ LD Local Dependence \\ MVPA Moderate to Vigorous Physical Activity \\ OECD Organisation for Economic Co-operation and Development \\ OR Odds Ratio \\ PFW Perceived Family Wealth \\ PES Parental Employment Status \\ PPS Probability Proportional to Size \\ SCN Saliva Thiocyanate \\ SD Standard Deviation \\ SES Socioeconomic Status \\ SEP Socioeconomic Position \\ UNICEF United Nations Children's Fund \\ VPA Vigorous Physical Activity \\ WHO World Health Organization \\ WLSMV Mean- and Variance-adjusted Weighted Least Square \\ WRMR Weighted Root Mean Square Residual \\ YRBSS Youth Risk Behaviour Surveillance System
}




\title{
CONTENTS
}

\author{
ABSTRACT \\ ACKNOWLEDGEMENTS \\ FIGURES AND TABLES \\ LIST OF ORIGINAL PUBLICATIONS \\ LIST OF ABBREVIATIONS
}

1 INTRODUCTION

2 A PUBLIC HEALTH PERSPECTIVE ON ADOLESCENT ALCOHOL

USE AND SMOKING

2.1 Health and social problems................................................................... 20

2.2 Factors related to alcohol use and smoking............................................ 21

2.2.1 Biological and physiological factors .............................................. 22

2.2.2 Psychosocial factors ................................................................... 22

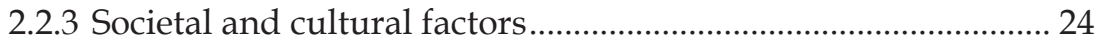

2.3 Measurements of alcohol use and smoking.......................................... 25

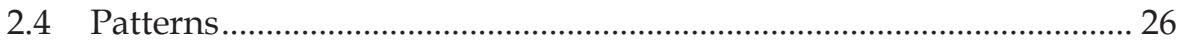

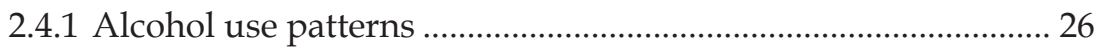

2.4.2 Smoking patterns............................................................................ 27

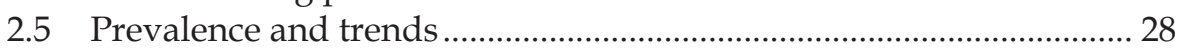

2.5.1 Prevalence and trends of alcohol use ......................................... 28

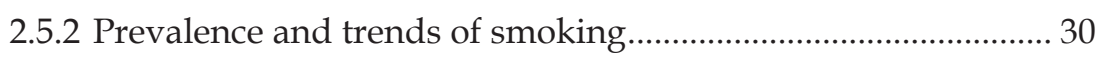

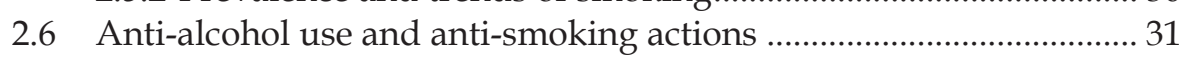

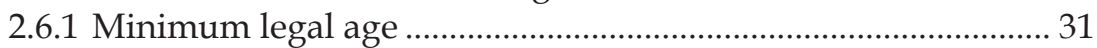

2.6.2 Controlling the affordability ........................................................... 32

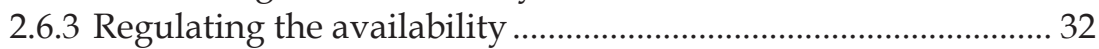

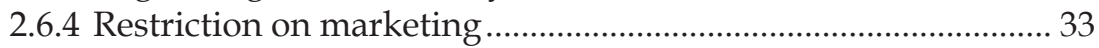

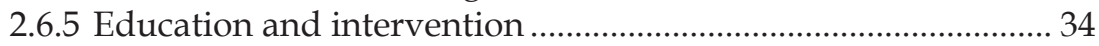

3 SOCIOECONOMIC INEQUALITIES IN HEALTH AND HEALTH

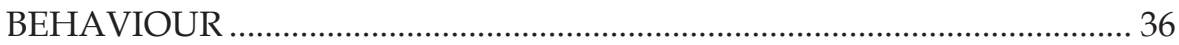

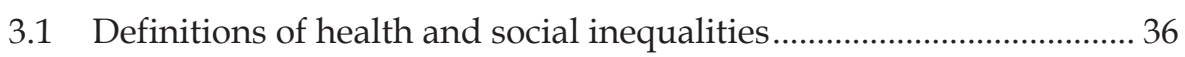

3.2 Explanations of socioeconomic differences in health ...........................37

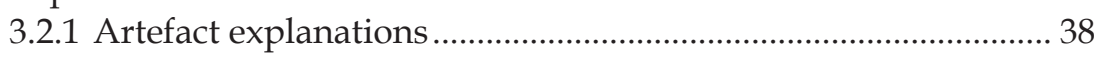

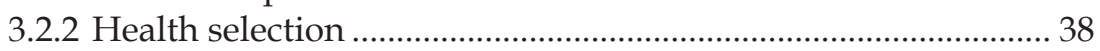

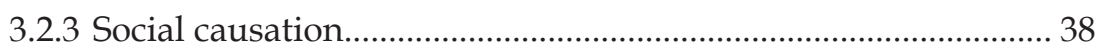

3.2.4 Different exposure and different vulnerability........................... 39

3.3 Measurement of socioeconomic status ................................................. 39

3.3.1 Measurement of adolescents' SES.................................................... 40

3.3.2 Family Affluence Scale (FAS) ..................................................... 41

3.4 Studies of socioeconomic inequalities in adolescent with focus

on alcohol use and smoking..................................................................... 42

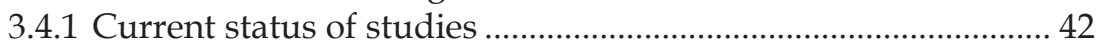


3.4.2 Socioeconomic inequalities in adolescent alcohol use

4 CROSS-NATIONAL COMPARATIVE RESEARCH ON

ADOLESCENT HEALTH BEHAVIOUR....................................................... 45

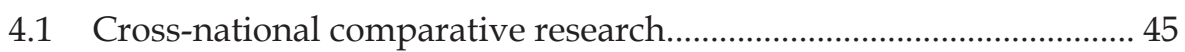

4.1.1 Purpose and significance................................................................ 45

4.1.2 Limitations and challenges ............................................................ 46

4.2 The Health Behaviour in School-aged Children (HBSC) study ......... 47

4.2.1 Research methods ........................................................................ 47

4.2.2 HBSC in non-member countries .................................................. 48

4.3 The application of the HBSC study in China........................................ 49

4.3.1 Current status of health behaviour of adolescents in China..... 49

4.3.2 Rationale and significance of HBSC study in China ................... 50

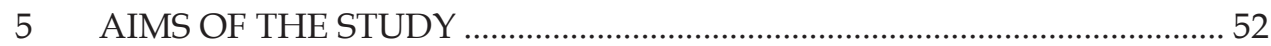

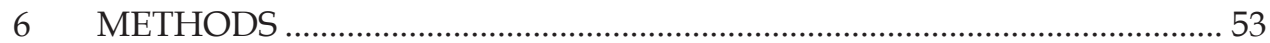

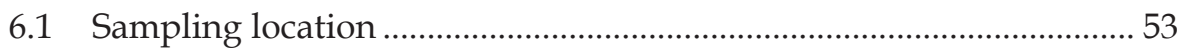

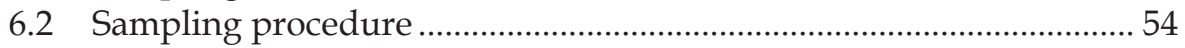

6.2.1 Test-retest reliability study in Beijing, China 2008 ….................. 54

6.2.2 Chinese HBSC linked project survey 2008 .................................. 55

6.2.3 Finnish HBSC survey 2006 ………………................................. 58

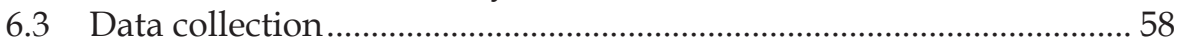

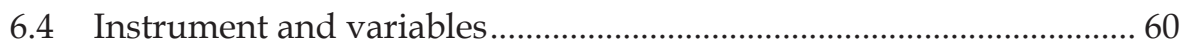

6.4.1 Selected items in test-retest reliability study..................................60 60

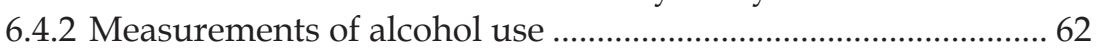

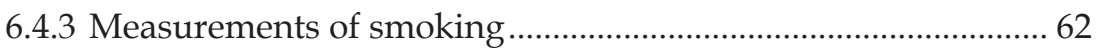

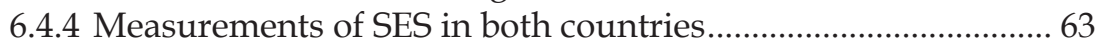

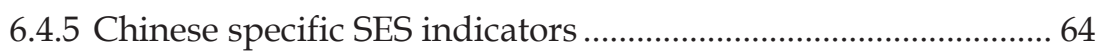

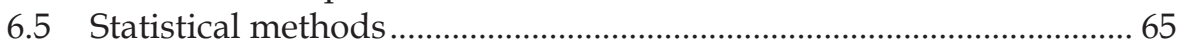

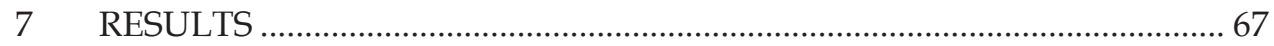

7.1 The test-retest reliability of selected survey items (Study I) ............... 67

7.2 The reliability and validity of the Family Affluence Scale

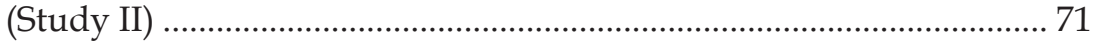

7.2.1 Test-retest reliability ...................................................................... 71

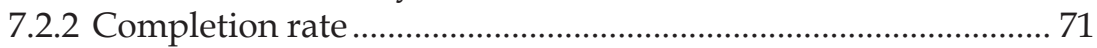

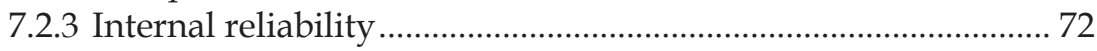

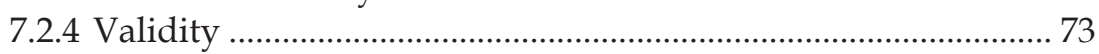

7.3 Alcohol use and socioeconomic differences (Study III) ........................ 76

7.4 Smoking and socioeconomic differences (Study IV) ............................ 78

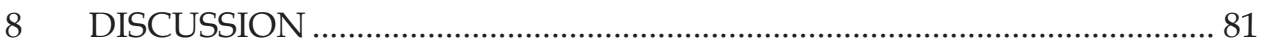

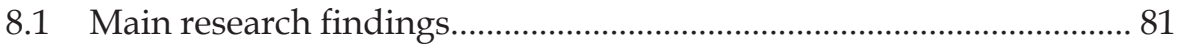

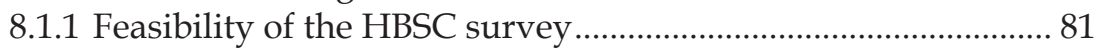


8.1.2 Test-retest reliability of selected HBSC survey items ..... 83

8.1.3 Reliability and validity of FAS II ............................................. 84

8.1.4 Socioeconomic differences in alcohol use............................... 87

8.1.5 Socioeconomic differences in smoking ..................................... 88

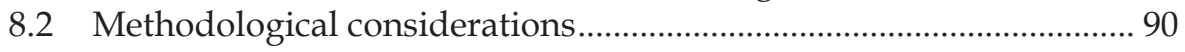

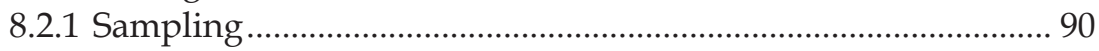

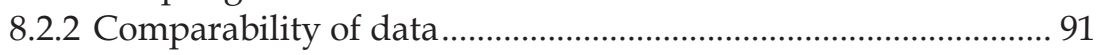

8.2 .3 Reliability and validity ............................................................ 93

8.2.4 Data analyses and interpretation ............................................ 94

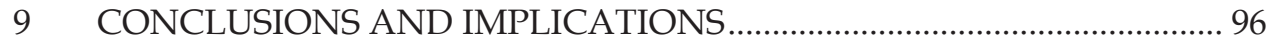

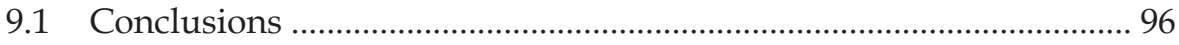

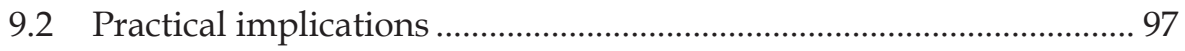

9.2.1 Contribution to the HBSC linked project in China................... 97

9.2.2 Reducing alcohol use and smoking of adolescents .................. 98

9.2.3 Future research challenges...................................................... 99

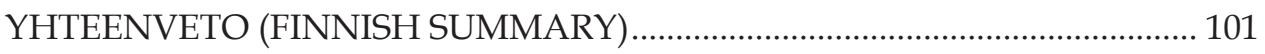

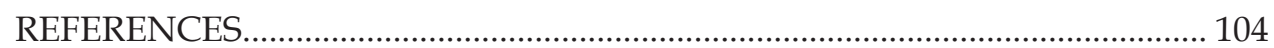

APPENDIX

ORIGINAL PAPERS 


\section{INTRODUCTION}

Adolescence is of great importance in the health of individuals, since during this phase major physical and psychological changes take place, in parallel with changes in social interactions and relationships. From a wider perspective, the health of adolescents is crucial in the sense that young people are central not only to the present but also to the future wealth and well-being of our societies. Social policies have been based on the need to invest in health and to improve trends in health and health behaviour. Thus, strategic information is needed to monitor young people's health, and from the data obtained, to implement and evaluate interventions aimed at promoting the health of adolescents. Occupying an important place among studies monitoring young people's health and health behaviour has been the project called Health Behaviour in School-Aged Children. This World Health Organization (WHO) collaborative cross-national study is one of the very first large-scale international surveys. It has been conducted over thirty years and has constructed an international research protocol for surveys in many different countries during that time.

From the global point of view, the situation regarding the health and health behaviour of young people in China cannot be ignored. Almost 400 million young people, aged less than 18, live in China, which is one of the world's most populated and most rapidly developing countries. Chinese economic development has continued over the last three decades, with accompanying improvements in Chinese standards of living and in the health care system. However, with regard to the health and health behaviour of Chinese young people, China, like other countries, still faces many problems. Numerous international and national studies have examined adolescent health behaviour in China (e.g. Anderson Johnson et al. 2006; Du et al. 2002; Liu et al. 2005; Xing et al. 2002, 2006). Nevertheless, very few of the studies have given a comprehensive profile of the health behaviour of Chinese young people or addressed the health behaviour of Chinese adolescents in a manner that would give a proper basis for comparison.

In 2007, the China Institute of Sport Science (CISS) started to conduct a comparative study on adolescent health behaviour applying the HBSC research 
protocol. In this it collaborated with the Finnish HBSC research team, thus making it possible to expand the HBSC study to China, and at the same time, to develop the HBSC further as a research instrument. The HBSC study is based on students' self-report measures (a procedure never previously applied on the mainland of China); hence, in order to ensure the feasibility, reliability, and validity of the HBSC in China and to prepare for the future Chinese HBSC linked project, the first aim of the study reported here was to examine the possibilities for implementing the HBSC survey in China, and to calculate the reliability of selected survey items in the Chinese context.

Among the health behaviours measured in the HBSC survey, health-risk behaviours, such as alcohol use and smoking, have been a global concern. Alcohol consumption in China has increased rapidly among the general population (WHO 2004a), including among young people, and this has been seen as a serious potential problem (Hao et al. 2005). A large population-based study, carried out in eighteen provincial capital cities in 2004, indicated that more than half of the Chinese students (aged 13 to 18) surveyed had used alcohol, while almost one third of them had initiated alcohol use before 13 years of age (Xing et al. 2006). Meanwhile, the prevalence of tobacco smoking in China is responsible for an enormous disease burden, posing a significant challenge to the Chinese public health system (Gu et al. 2009). According to statistics, there are currently about 15 million smokers, and 40 million people trying out smoking behaviour, among the 130 million adolescents aged 13 to 18 in China (Wang et al. 2011). Here it should be noted that in terms of the recent HBSC survey report (Currie et al. 2012), the prevalence of alcohol use and smoking among Finnish young people is even higher than among Chinese adolescents of the same age group. Regardless of the differences in culture, or in the economic, geographical, and political context, it appears that drinking and smoking are common among adolescents in both China and Finland, making the need to decrease adolescent alcohol use and smoking a public health priority.

Various studies have demonstrated the associations between socioeconomic status and health and health behaviour among adolescents. It has been argued that even if there are no consistent socioeconomic differences in health among adolescents, there may be socioeconomic differences in health-risk behaviours during this period of life (Tuinstra et al. 1998). The various adolescent health-risk behaviours in question have been shown to have an adverse effect on health in the long term (Pietilä et al. 1995), implying that socioeconomic differences in health-risk behaviours in adolescence constitute one of the predictors of socioeconomic health differences in adulthood. However, the research findings concerning socioeconomic inequalities in alcohol use and smoking are inconsistent, and most of the relevant studies have been conducted in North America and Europe.

Reducing the alcohol use and smoking of adolescents is a priority in the public health sector globally. A challenge for policy makers and health promoters is how to allocate limited resources to the design and implementation of effective intervention programmes. If greater efforts are to be made, it will be 
important to design effective policies targeted at vulnerable populations. There is clearly a need for research addressing socioeconomic inequalities in alcohol use and smoking among adolescents. Such research may not merely contribute to science but also provide evidence regarding the practical implications of reducing adolescents' alcohol use and smoking. Thus, the second aim of the study reported here was to investigate and compare socioeconomic inequalities in alcohol use and smoking among adolescents in China and Finland.

In the present comparative study, Finland represents a developed European country with a well-developed social welfare system and few socioeconomic inequalities, while China represents a developing Asian country with an undeveloped social welfare system and greater socioeconomic inequalities. Research comparing these two countries with respect to socioeconomic inequalities in the alcohol use and smoking of adolescents can provide evidence on whether socioeconomic status influences adolescents' alcohol use and smoking, independently of the surrounding cultural and political context. The outcomes of this comparative study have major potential significance. In the first place, from a theoretical point of view, the findings from this study can broaden our understanding of socioeconomic inequalities with respect to alcohol use and smoking in adolescence. In addition, this study can provide valuable information on the methodological development of the HBSC instrument beyond Europe and North America. In practical terms, from the health promotion point of view, the evidence-based findings from this study can provide valuable information relevant to the planning and implementation of programmes aimed at different target populations, thus aiding policy makers and health practitioners in each of the countries concerned. 


\section{A PUBLIC HEALTH PERSPECTIVE ON ADOLESCENT ALCOHOL USE AND SMOKING}

Adolescents, usually defined as young people from ages 10 to 19 , are in the critical period of human growth and development in many aspects, such as biological, cognitive, psychological, and social development (Choudhury et al. 2006; Lerner \& Steinberg 2004; Marshal 1978; Steinberg 2008). The World Health Organization identified that adolescence represented one of the key transitions in the life span (WHO 2013). During this phase, the health behaviour and future patterns of adult health are established (Sawyer et al. 2012). Of special concern is the fact that many adolescents face pressure to engage in health-risk behaviours such as using alcohol and smoking (WHO 2013). From a public health perspective, therefore, understanding and then preventing those risk behaviours among adolescents are of vital importance.

In this chapter, the health and social problems caused by adolescents' alcohol use and smoking will be introduced and discussed. The factors and measurements that relate to alcohol use and smoking of young people, as well as their patterns, will then be reviewed respectively. In the final part of this chapter, prevalence and trends of alcohol use and smoking of adolescents, and policies, strategies and interventions to reduce them will be described, with special focus on the situations in Chinese and Finnish contexts.

\subsection{Health and social problems}

Alcohol use and smoking are global problems which have many negative consequences. On an individual level, their related effects are harmful to the person's health and consequently contribute to morbidity and mortality. According to the WHO statistics, globally, 2.5 million deaths are the consequence of harmful use of alcohol (WHO 2011a) and approximately one person dies every six seconds due to tobacco (WHO 2012a). On a societal level, for instance, the use of alcohol generates obstacles for social development, e.g. violence, property dam- 
age and social cost (Klingemann \& Gmel 2001), and raises the financial burden on the society from associated and related illness.

The effects caused by alcohol use and smoking during adolescence are even worse, compared to later adulthood. The use of alcohol in adolescence can be a major problem resulting in significant disability and premature death (Rehm et al. 2001; WHO 2004a). It has also been documented that adolescent alcohol use predicts alcohol-related problems in young adulthood and later life (Duncan et al. 1997). In addition, alcohol use is a significant contributor to injury in adolescence (Zeigler et al. 2005). Particularly, abuse of, or dependence on alcohol, in adolescents is associated with alcohol poisoning (Perkins 2002), motor vehicle crashes (National Highway Traffic Safety Administration 2000), risky sexual behaviours (Tapert et al. 2001), drowning (Cummings \& Quan 1999), other drug use (Arria et al. 1995), suicidal ideation and attempts (Schiling et al. 2009) and completed suicide (Pirkola et al. 1999). Additionally to those negative effects on physical health caused by alcohol use in adolescence, research has also shown that adolescent alcohol abuse is associated with various psychosocial problems, such as family dysfunction (Clark et al. 1998) and academic difficulties (Clark \& Kirisci 1996).

According to the new edition of The Tobacco Atlas (Eriksen et al. 2012), ‘50 million people have been killed as a result of using tobacco in the past decade'. It has been stated that smoking harms nearly every organ of the body, causing many diseases and reducing the health of smokers in general (U.S. Department of Health and Human Services 2004). In spite of the fact that most regular smokers are adults, similar to the alcohol use, smoking among adolescents has also been a major concern because it poses many health hazards. Sufficient evidence has demonstrated that cigarette smoking during adolescence produces significant negative somatic health outcomes among young people, including cough and phlegm production, respiratory illness, low back pain (Mikkonen et al. 2008), decreased physical fitness, and increased risk of causing many chronic diseases (U.S. Department of Health and Human Services 2012). Meanwhile, evidence is emerging that smoking is related with various developmental and mental health disorders that effect young people. For instance, a systematic review of longitudinal studies suggested that adolescents' smoking can predict depression (Chaiton et al. 2009). In addition, Riala and her colleagues (2007) found that regular smoking in adolescence increases the subsequent risk of suicide. Except for those short and long term effects on health, tobacco smoking is often considered as a 'gateway drug', and is associated with subsequent alcohol and illicit drug use among adolescents (Duncan et al. 1998).

\subsection{Factors related to alcohol use and smoking}

In addition to the studies on the effects on health and society caused by adolescents' drinking and smoking, researchers have been investigating the factors related to these behaviours in order to understand how various risk and protec- 
tive factors are associated with alcohol use and smoking among young people. Those factors can be summarized from previous empirical literatures as biological and physiological factors and psychosocial factors from individual level, and as social and cultural factors from the macro-level (Donovan 2004; Rhee et al. 2003; Saffer \& Dave 2006).

\subsubsection{Biological and physiological factors}

The first biological and physiological factor we should consider when examining alcohol use is genetic risk. Most research addressing genetic risk of alcohol use was investigating an adult sample and the findings may not therefore be transferred directly to adolescents (Schulte et al. 2009). Studies focused on adolescence demonstrated that the initiation of alcohol use and social drinking were only minimally related to genetic risk, however, problematic use, was demonstrated to be highly heritable (Pagan et al. 2006). Furthermore, it has been reported that the genetic risk for alcohol use is highly correlated with friends' alcohol use, which highlighted the sophisticated interaction of genetic and environmental variables on adolescents' drinking behaviour (Fowler et al. 2007). Neurobiological factors are another physiological vulnerability for the development of alcohol use disorders (Tapert \& Schweinsburg 2005). The P300 eventrelated potential (ERP), reflecting attention and cognitive processing, were found to have reduced amplitudes among children with a family history of alcoholism (Hill \& Steinhauer 1993). Based on the results of Finnish Twins Study, it had been documented that both genetic and environmental factors contribute to the development of patterns of alcohol use during adolescence, and once drinking has been initiated, genetic influences assume increasing importance across development (Rose et al. 2001).

Cigarette smoking is the most common use of tobacco among every population group, and nicotine, as one of the indispensable components of cigarettes, is highly addictive. Researches concentrating on the biological influence of smoking therefore focused on genetic factors that influence initiation, dependence and cessation of nicotine intake (Lerman et al. 2007). Several studies with twin samples have shown that approximately $60 \%-70 \%$ of the variability in nicotine dependence and smoking persistence is due to genetic influences (Broms et al. 2006; Li et al. 2003; McGue et al. 2000) and over 50\% of the variance in the ability to quit smoking was attributed by genetic factors (Xian et al. 2003; Xian et al. 2005). Meanwhile, a twin study also provided the evidence for moderate to substantial genetic influences and modest to moderate shared environmental influences on initiation, use, and problem use of tobacco (Rhee et al. 2003).

\subsubsection{Psychosocial factors}

In addition to biological/physiological factors, previous studies have identified many psychosocial factors associated with substance use of adolescents. Those psychosocial factors can be summarized as four categories (Tyas \& Pederson 1998): sociodemographic factors (age, gender, ethnicity/race, family structure, 
socioeconomic status, country/region, and urban/rural residence), environmental factors (family domain, peer domain, school domain and community domain), behavioural factors, and personal factors.

Numerous international and national studies have shown the sociodemographic difference in substance use of adolescents (e.g. Currie et al. 2012; Eriksen et al. 2012; Hibell et al. 2012; WHO 2011b, c) and some facts have been well documented. For instance, substance use, in general, is more common among boys than among girls (Currie et al. 2012; Hibell et al. 2012) - although, this gap narrowed recently - (Johnston et al. 2011), the percentage of drinking and smoking increases with age among schoolchildren (Currie et al. 2012), and the prevalence varies in different countries or regions (Eriksen et al. 2012; WHO $2011 b, c)$. Socioeconomic status influences on alcohol use and smoking were also investigated by many researchers although the results showed inconsistent relationships (Gecková et al. 2005; Liu et al. 2013; Melotti et al. 2011; Richter et al. 2006; Simetin et al. 2010).

Environmental factors play an important role in contributing to the influence on substance use of adolescents. In the family domain, family history of substance use has been found as a strong risk factor predicting alcohol use and smoking among adolescents (Biglan et al. 1995; Murphey et al. 2012; Peterson et al. 1994). Furthermore, many aspects of family culture, like poor family management practice (Peterson et al. 1994), the absence of bonding to family (Brook et al. 1990), parental attitude (Botvin et al. 1994), family environment (Farrell et al. 1992), and sibling's behaviour and influence (McGue et al. 2000) are additional family factors (Ryan et al. 2010) related to adolescents' substance use.

In the period of adolescence, social relations become more wide and important to the individual, and therefore the peer group becomes more important for the adolescent than the family, especially with regards to substance use, which is mainly initiated, maintained and shaped in the peer group (La Greca et al. 2001). Previous studies suggest that peer influence is a crucially important factor since studies have consistently found a positive relationship between substance use of peers and substance use of young people (Björkqvist et al. 2004; Hawkins et al. 1992; O'Loughlin et al. 2009). A systematic review on psychosocial factors of adolescent alcohol initiation found that the most consistent antecedent risk factors were parental and peer approval and models for drinking (Donovan 2004). In addition to family and peer domains, evidences have been provided regarding factors within school and community domains can influence students' substance use (Bisset et al. 2007; Wray-Lake et al. 2012).

Three major categories of behavioural factors can contribute to smoking and alcohol use of adolescents. First were those factors related to school, for instance, lower level of academic performance was consistently associated with the status of smoking (Bewley \& Bland 1977; Audrain-McGovern et al. 2004) and alcohol use (McGue et al. 2001). The second category comprised other risk behaviours, that show both pairwise associated and accumulative effects on alcohol use and smoking among adolescents. For instance, early onset of drinking was found significantly related to participating in many of the risk behav- 
iours, like smoking, among adolescents (Calvert et al. 2010). The third and last category of behavioural factors is lifestyle. Longitudinal research has implicated those behavioural risk factors for the initiation of adolescent alcohol use and/or smoking, to include greater levels of watching TV (Robinson et al. 1998), and frequent dieting (Krahn et al. 1996). In addition, prior involvement in delinquent behaviour was found as one of the most consistent behavioural risk factors for initiation of alcohol use of adolescents (Stice et al. 1998).

Apart from those above-mentioned psychosocial factors, previous studies have also identified the relationship between personal factors and substance use of adolescent. These factors can be summarized as personality characteristics (Schulte et al. 2009; Wijatkowski et al. 1990); motivational factors, such as stress (Finkelstein et al. 2006; Patton et al. 1996) and depression (Diego et al. 2003; Otsuki 2003), and personal resources, such as coping skills (Wills et al. 2001), self-esteem (Donnelly et al. 2008; Jimenez et al. 2008), attitudes to substance use (Barkin et al. 2002), knowledge of health effects of substance use (Yeretzian \& Afifi 2009), and personal health concerns (Jensen \& Overgaard 1993).

\subsubsection{Societal and cultural factors}

Beyond individual level factors are the broader societal and cultural factors that play important roles in influencing substance use of adolescents. Although the use of substances is very common across most societies, the patterns of adolescents' alcohol use and smoking vary in different countries or regions due to the societal differences which impact on people's norms, beliefs, values and expectations on substance use, as well as the availability of substances for people to access.

Substance use serves a symbolic and/or functional role in certain cultures (Durrant \& Thakker 2003), and hence it is affected by the cultural factors. There has been a long history of alcohol consumption in most countries, and it is embedded in many cultures. From the earliest recorded use of alcohol, drinking has been a social activity and served a role in various ways in different cultures (The Social Issues Research Centre 1998). For instance, traditional Chinese cultural norms encourage social drinking, especially with family/relatives and friends (Cochrane et al. 2003). Drinking alcohol and being drunk has been part of male initiation to adulthood in many civilisations (Godeau 2000).

Cigarette smoking has also been accepted into culture although it did not begin to become widespread until the late 19th century (Randall 1999), therefore making the popular history much shorter than alcohol use. People view cigarette smoking as a source of relaxation in times of trouble and an effective means of building association between individuals who indulge in the habit. As a cultural attribute, smoking is often portrayed in literature and movies with an image of people who have a sense of glamour and romance, or by heroes with a cigarette in their hand. One recent piece of research found that exposure to smoking in movies is linked to adolescent smoking (Morgensten et al. 2011). 


\subsection{Measurements of alcohol use and smoking}

Studies on alcohol use and smoking may focus on different issues and perspectives due to the various aims of the studies. They do although have one thing in common, that is the assessment of alcohol use and smoking. Various methods of assessing and measuring substance use behaviours have been employed in previous studies, which can be generally divided into subjective measures like self-report questionnaires, and objective measures like biological tests.

The standard approach of measuring adolescent substance use is through self-report questionnaires. All large scale international and national studies aiming on investigating the prevalence and trends of substance use collect information through self-report questionnaires, including the Global Youth Tobacco Survey (GYTS), the Global School Health Survey (GSHS), the Youth Risk Behaviour Surveillance System (YRBSS), the European School Survey Project on Alcohol and Other Drugs (ESPAD), as well as the HBSC study. In general, items regarding frequency, amount, time, onset of alcohol use and smoking were included in self-reported questionnaires, as well as other items regarding some other concerns in light of the aim of the study.

Richter and Johnson (2001) reviewed various means of assessing substance use behaviour and stated the following advantages of self-report measurement: 1) relatively easy to administer to large samples; 2) can be administered simultaneously in several different locations; 3) responses are easily quantifiable and analysable; 4) possibility to ask on many different areas of interest; 5) relatively inexpensive to conduct; 6) can be administered in different ways, such as in person, via mail, or via the Internet; 7) the respondent can choose which items he or she wants or does not want to answer to ensure the data collection is ethically acceptable. Despite of these advantages, Richter and Johnson (2001) also pointed out the disadvantages of self-report measures in light of their reliability and validity due to their subjective nature, which included demand characteristics, social desirability, research bias, and the wording of self-report items. Furthermore, Brener and colleagues (2003) reviewed factors affecting the validity of self-report health-risk behaviour, including alcohol use and smoking, among adolescents and found that although self-report measures are affected by both cognitive and situational factors, the validity of self-reports of behaviours are not threatened.

However, one important issue concerning self-reported measurement of substance use behaviour should be noted. Through self-report assessment, it is usually easy to underestimate the sensitive or risk behaviours, including adolescent substance use (Tourangeau \& Smith 1996; Turner et al. 1998), which may be due to errors in reporting and conscious attempts to provide inaccurate information for embarrassing or socially undesirable behaviours (Bradburn 1983). This issue is complicated and even contradictionary regarding alcohol use and smoking, especially when the study population is adolescents. On one hand, since drinking alcohol and smoking at young age are usually not encouraged or 
even forbidden, which can be seen as social undesirable behaviour and therefore it might be underreported. On the other hand, in many countries, drinking alcohol and smoking cigarettes can be seen as social desirable behaviour among adolescents themselves and therefore they might have been over reported.

Apart from the most commonly used subjective measures of substance use, biological tests can also be used in assessment. These tests include the most commonly used electrochemical sensor devices, breathalysers and transdermal (sweat) sensors, to detect ethanol (alcohol), and saliva thiocyanate (SCN) analysis (Leffingwell et al. 2013; Luepker et al. 1981), or Mouthpiece-based computerized devices (Blank et al. 2009) to document smoking. Since the objective measures of substance use are more valid, researchers usually use them as an external validation for the objective measures and the discordance between selfreport and biological measures is generally assumed to reflect biases in selfreport due to social desirability (Dolcini et al. 1996). A meta-analysis compared the validity of self-reported tobacco use to biochemical measures of tobacco use in 30 smoking studies, and the results revealed that self-reported measures possessed generally high levels of sensitivity and specificity, though student selfreports had lower sensitivity than general population (Patrick et al. 1994). However, it is not possible to measure long term habits of health behaviour using the objective measures due to their nature.

\subsection{Patterns}

\subsubsection{Alcohol use patterns}

The term drinking patterns discussed here refers to the frequency and amount of alcohol consumption, the type of alcoholic beverages, the frequency of drunkenness, the onset of alcohol use, and the onset of drunkenness.

The amount of alcohol absorbed and diffused into the bloodstream, and the extent of its effects on the human body depends on how much one has drunk. Thus, the frequency and amount of alcohol consumption are associated with alcohol-related risk behaviours (Munro \& Learmonth 2004). Frequency of alcohol use has been extensively employed as an indicator of measuring drinking behaviour and has been proved to be an empirically supported brief screen for use with adolescents to identify alcohol-related problems (Chung et al. 2012; Clark et al. 2006).

Another important issue is what kind of alcohol adolescents drink. There are many varieties of alcoholic beverages, with different levels of alcohol content, such as beer, wine, and spirits. In accordance with previous studies, the most commonly reported beverage among young people in 36 European countries was beer, followed by wine and spirits (Hibell et al. 2012), which were all found to have negative effects on health (Hennekens et al. 1979). In addition, new alcoholic beverages, like alcopops and ciders which especially targeting the 
teenage market, are also becoming more and more popular among adolescents (Martin 2012).

An earlier onset of alcohol use is associated with various alcohol-related problems. For instance, early onset of adolescent alcohol use is often associated with problematic alcohol consumption (Andersen et al. 2003; Tucker et al. 2003), alcohol dependency (Andersen et al. 2003), frequent drinking, heavy episodic drinking and earlier onset of drunkenness (Chassin et al. 2002; Hill et al. 2000). The early onset of alcohol use usually refers to adolescents who have drunk any alcoholic beverage at age of 13 or younger (Currie et al. 2012; Hibell et al. 2012). It has been shown that in three-quarters of the ESPAD countries, at least half of the students are early onset drinkers (Hibell et al. 2012).

Drunkenness is defined as the state of being intoxicated by consumption of alcohol, to a degree that mental and physical facilities are noticeably impaired (Science Daily 2013). It is a risk factor in many injuries, and severe drunkenness can even turn into acute alcohol poisoning (Tator 2008). In addition, a drunkenness oriented drinking pattern in adolescence is a sign of current and future problems with alcohol, and a predictor of mental problems (Wechsler et al. 1998). The trend of adolescent drunkenness has increased, for example, in Finland (Lintonen et al. 2000), although the prevalence of alcohol use may remain stable. The recent ESPAD report showed that on average, nearly half of the students $(47 \%)$ reported that they had been drunk at least once during their lifetime (Hibell et al. 2012).

Compared with the early onset of drinking, early onset of drunkenness is more likely to cause negative effects on adolescents' health. For instance, a recent study showed that early onset of drunkenness, not early onset of drinking, was a strong predictor for all five measured problem behaviours (tobacco use, cannabis use, injuries, fights, and low academic performance) in 15-year-old schoolchildren in 38 European and North American countries (Kuntsche et al. 2013). Furthermore, delayed onset of drunkenness was found to be a protective factor for adolescent alcohol misuse (Thomas et al. 2000).

\subsubsection{Smoking patterns}

The term smoking patterns refers to the frequency of smoking, type of tobacco use, and the onset of smoking. It has been suggested that even a single cigarette smoked, or exposure to second hand smoke can have health consequences, since even small amounts of the chemical in cigarettes can cause rapid inflammation in the endothelium, or lining, of blood vessels and in the lungs (U.S. Department of Health and Human Services 2010). In adolescents, nicotinedependence was found to be associated with smoking frequency $\left(\mathrm{O}^{\prime}\right.$ Loughlin et al. 2003). Daily or weekly smoking is usually considered as regular smoking, which is a big threat for one's health. According to the HBSC study, on average, nearly one-fifth of students aged 15 years in 38 participating countries are weekly smokers (Currie et al. 2012).

The second issue of adolescent smoking patterns regards what they smoke. People use tobacco in various ways, but the global predominance is the use of 
manufactured cigarettes (Eriksen et al. 2012). It has been reported that more than half of the adolescents in the ESPAD countries had smoked at least one cigarette, and the mean number of smokers among boys and girls were very close (Hibell et al. 2012). Most research on adolescent tobacco use focuses on cigarette smoking. It should be noted that although cigarette smoking is the most popular way of using tobacco among adolescents, smokeless tobacco use, such as the use of chewing tobacco, moist stuff, water pipes etc., is also a big problem among young people (Tomar 2003). Smokeless tobacco use is still a harmful alternative to smoking cigarette and has little effect on quitting smoking (Tomar 2003).

Onset of smoking occurs largely during adolescence, and compared to adult smoker, the young smoker is more vulnerable to becoming addicted to nicotine. It is therefore harder for the adolescent to subsequently quit smoking (U.S. Department of Health and Human Services 2012). The smoking initiation of the adolescent can be influenced by many individual, social and cultural factors, such as parents (Gilman et al. 2009), and peers (Moffitt 2006), both of which have been discussed in the previous section (see factors influencing alcohol use and smoking). Studies usually define young people who have smoked a cigarette at the age of 13 or younger as an early onset smoker (Currie et al. 2012; Hibell et al. 2012). Using this definition, nearly one-third of students in ESPAD countries, (Hibell et al. 2012) and nearly one-quarter of students in HBSC countries are early onset smokers (Currie et al. 2012). Thus, delaying the initiation of smoking among young people is important when reducing the prevalence of smoking in both adolescent and adult populations.

\subsection{Prevalence and trends}

Since there is not a single country in the world where young people do not drink or smoke, adolescents' alcohol use and smoking is a global issue, and recognized as a major public health problem in many countries (Ahlström \& Österberg 2004; Lando et al. 2010). However, the prevalence and trends of alcohol use and smoking of adolescents vary enormously not only among regions and countries, but also over different population and gender groups.

\subsubsection{Prevalence and trends of alcohol use}

According to the WHO fact sheet (2011a), 14\% of adolescent girls and 18\% of boys aged 13-15 years in low- and middle-income countries are reported to use alcohol. The prevalence of alcohol use of adolescents is even more common in high income countries. For instance, a recent YRBSS report showed that 38.7\% of high school students in the U.S. had drunk alcohol during last 30 days (Eaton et al. 2012). The recent ESPAD survey report on substance use among 15-16year-old students in 36 European countries revealed that the corresponding av- 
erage figures for alcohol use of adolescents in the past 12 months and the past 30 days are $79 \%$ and $57 \%$ respectively (Hibell et al. 2012).

The prevalence of adolescent drinking behaviour has changed over time. The WHO Global Survey on Alcohol and Health (2011b) investigated the fiveyear trend of under-age drinking and found that out of 73 surveyed countries, $71 \%$ indicated an increase, $4 \%$ a decrease, $8 \%$ were stable and $16 \%$ showed inconclusive trends. In general, alcohol use is on the rise among adolescents and young adults in many countries (Lancet 2008; WHO 2007), except that there were small decreases from 2003 through 2007 to 2011 in some European countries (Hibell et al. 2012). With the emergence of alcoholic carbonated drinks, frequent drinking, early onset of alcohol use and drunkenness increased among young people (Kraus et al. 2010). In addition, although drinking alcohol is more prevalent among boys than among girls (Currie et al. 2012; Hibell et al. 2012), the gap narrowed recently (Johnston et al. 2011), and no gender difference in the frequency of drunkenness were even found, whereas heavy episodic drinking was more prevalent among boys (Hibell et al. 2009).

It has been stated that Europe has the highest alcohol consumption rates in the world (Anderson \& Baumberg 2006), and the use of alcohol among young people in European countries is very common and more popular than the use of any illegal drugs (Hibell et al. 2000). Like in other European countries, alcohol drinking of school-aged children is also very common in Finland. Findings from The Adolescent Health and Lifestyle Survey (AHLS) indicated that at the end of last century alcohol drinking was common among fourteen-year-olds with about two-thirds drinking at least occasionally (Lintonen 2001) but alcohol abstinence has increased since the beginning of the 2000s and the increases continued in 2013 among all age and gender group (Kinnunen et al. 2013; Lintonen et al. 2013). Based on the international ESPAD survey results from 1995 to 2007, although heavy episodic drinking among Finnish adolescents has significantly decreased during the 2000s (Metso et al. 2009), there were still nearly half (48\%) of Finnish 15-year-olds who had drunk in the past 30 days (Hibell et al. 2012). In Finland, adolescents' drinking habits are drunkenness-orientated. The most recent results from the HBSC study revealed that although the weekly alcohol consumption of Finnish school-aged children is below the HBSC average, the proportion of 15-year-olds who reported they have been drunken at least twice ranked 12th among 43 countries (boys 37\% and girls 44\%) (Currie et al. 2012). In addition, the early onset of alcohol use or drunkenness is typical among Finnish adolescents. For instance, nearly one fifth of 15 year old Finnish students reported that their first experience of getting drunk was at age 13 or younger (Currie et al. 2012).

Since the 1980s, the alcohol consumption in China has increased rapidly among the general population (WHO 1999, 2004a) as well as among young people (Hao et al. 2005). The China Adolescent Behavioural Risk Factor Survey (CABRFS), a large population-based study which was carried out in 18 provincial capital cities indicated that more than half of students aged 13 to $18 \mathrm{had}$ ever used alcohol (boys: 58.6\%, girls: 44.3\%), and almost one third of Chinese 
students initiated alcohol use before 13 years of age (Xing et al. 2006). Moreover, $22.1 \%$ of 15 -year-old boys and $12.7 \%$ of 15 -year-old girls reported that they had been drunk in past 12 months (Ji 2007).

\subsubsection{Prevalence and trends of smoking}

Globally, it is estimated that 150 million young people currently use tobacco (WHO 2011c, d). The prevalence of smoking in adolescence varies widely around the world. Nearly three decades ago, Lopez et al. (1994) proposed a four-stage model of cigarette smoking in a country: stage 1-low smoking prevalence in men and very low in women; stage 2-smoking prevalence in men rapidly increases while it increases slowly in women; stage 3-smoking prevalence in men decline and in women peaks and then begins to decline; stage 4smoking prevalence in both men and women continue to decline. In most highincome countries, the rate of smoking is keeping steady or decreasing. For instance, in U.S., $18.1 \%$ of high school students had smoked cigarettes during the last 30 days (Eaton et al. 2012), and in Europe, on average, 54\% of the 15 -yearold students reported that they had smoked cigarettes at least once and $28 \%$ of participants had used cigarettes during last 30 days (Hibell et al. 2012).

However, no matter at which stage, reducing adolescent smoking is always one of the priorities of public health concern in each and every country. At present, Finland, as other Northern European countries, has reached the final stage at which the overall prevalence of smoking is decreasing. For instance, according to the AHLS, experiments of smoking and daily smoking have decreased among Finnish adolescents during the study period 1977-2011 in all age groups and both genders (Raisamo et al. 2011) and the decreasing trend continued also over the past two years (Kinnunen et al. 2013). The past two Finnish HBSC surveys (2006 and 2010) indicated that one fifth of 15-year-old Finnish schoolchildren reported smoking weekly, and one third of them reported first smoking at the age of 13 or younger (Currie et al. 2008a, 2012).

Contrary to high-income countries, with westernization and economic development, adolescents' smoking is increasing and becoming more popular in developing countries (Eriksen et al. 2012). China, with a total 320 million smokers, is the world's largest tobacco producer and tobacco consumer (Zhang \& Cai 2003). The prevalence of adolescents' smoking has increased dramatically in China since the 1990s (Ji 2007). The China Seven Cities Study (CSCS) in 2001 showed that in the past-month smoking was reported by $9 \%$ among middle school students, $8 \%$ among academic high school students, and $26 \%$ among vocational high school students (Anderson Johnson et al. 2006). In a Youth Risk Behaviour Survey in Guangzhou, nearly one fifth of students aged from 12 to 19 years reported they had tried smoking (Wang et al. 2009). Though the absolute percentage of young smokers in China is still less than in many developed countries, and varies across age, gender and area, the increasing trend of cigarette smoking in China should not be neglected since young smokers are more likely to become habitual smokers in adulthood (Mistry et al. 2011), and the smoking prevalence in China has not reached the stable stage yet. For instance, 
it was reported that 80,000 Chinese young people become new smokers everyday (Zhang \& Cai 2003).

\subsection{Anti-alcohol use and anti-smoking actions}

Alcohol use and smoking of adolescents are influenced by wide-ranging factors. In general, to reduce the harm caused by substance use, actions include national or regional policies on minimum legal age, controlling the affordability (tax and price), regulating the availability, restrictions on marketing, as well as education and intervention (Babor et al. 2010). Numerous studies have consistently shown that price, taxation, regulations and policies on alcohol and tobacco have a substantial influence on substance use of adolescents (e.g. Chaloupka 2003; Emery et al. 2001; Muller et al. 2010; Saffer \& Dave 2006). Though there is still evidence reported that alcohol policy changes seem not to have had noticeable influence on drinking alcohol or drunkenness among Finnish adolescents (Lintonen et al. 2013), anti-substance use actions still need to be addressed to prevent the increasing trend emerging.

Most countries globally have taken various actions aimed at reducing substance use. Internationally, the WHO launched the Framework Convention on Tobacco Control (FCTC) in 2003 (WHO 2005) and the global strategy to reduce harmful use of alcohol use in 2010 (WHO 2010). The premise of this section is to summarize the policies, strategies and interventions on reducing alcohol use and smoking among young people, and to describe the situation in both China and Finland.

\subsubsection{Minimum legal age}

Minimum legal age concerning adolescent substance use usually means the minimum legal age at which people can purchase alcoholic beverages and tobacco, or in other words, the minimum legal age of people to whom the retailers can sell alcoholic and tobacco products. Increasing the minimum age for the legal purchase of alcohol and tobacco was associated with reduced regular substance use among youth (Coate \& Grossman 1988; Millett et al. 2011). Therefore, WHO FCTC (WHO 2005) clearly stated that the sales of tobacco products to persons under the age set by law or eighteen is prohibited.

Most countries (almost all European and North American countries) currently have legal age limits on sales of alcoholic beverages and tobacco products ranging from 16 to 21 years (Ahlström \& Österberg 2004; WHO 2011b). In China, the merchants are not allowed to sell alcoholic beverages to people aged less than 18 years (Ministry of Commerce of China 2005). However, there is still not any legislation on minimum age for purchasing cigarettes at the present time. In Finland, the minimum legal age is 18 years for purchasing or selling tobacco products (Ministry of Social Affairs and Health of Finland, 2010), 18 years for purchasing light alcoholic beverages $(<22 \%$ Alcohol by volume $(\mathrm{ABV}))$, and 20 
years for purchasing mild alcoholic beverages $(>=22 \%$ ABV) (Ministry of Social Affairs and Health of Finland 1997).

\subsubsection{Controlling the affordability}

There are extensive evidences to support the association between affordability of alcohol and tobacco products and the consumption of alcohol and smoking and their related harm (WHO 2011a, 2012a). Taxes and prices of alcohol and cigarettes are the most commonly used means to control the affordability of substances, and therefore to reduce the prevalence of drinking and smoking, especially among young people. A meta-analysis of 1003 estimates of the tax/price-consumption relationship from 112 studies (Wangenaar et al. 2009) established that taxes and prices of alcoholic beverages are related inversely to drinking prevalence. In addition, the WHO fact sheet on tobacco (2012a) stated that 'a tax increase that increases tobacco prices by $10 \%$ decreases tobacco consumption by about $4 \%$ in high-income countries and by up to $8 \%$ in low-and middle-income countries'.

Many countries have already levied special taxes and high prices on alcoholic beverages and cigarettes. In Finland, the alcohol tax rates have been increased four times in the last ten years, and the beer tax, which accounts for 60$80 \%$ of the per-litre retail price of beer, is the highest in the EU (Finnish Federation of the Brewing and Soft Drinks Industry 2012). According to Mäkelä \& Österberg (2009), the high price of alcoholic beverages is one of the key means in alcohol consumption control in Finland. Alcohol and cigarette manufacturing are important industries in China and the taxes from them, based on the newest statistics, represents more than one tenth of the total national annual revenue (National Bureau of Statistics of China 2013). The tax rates of alcohol and cigarettes have also been increased in China in last decade, while it has been argued that the increases of taxes is aimed at increasing the national revenue rather than reducing the substance use of Chinese people (Zhou 2009). Although the prices of alcoholic beverages and cigarettes in China ranges a lot depending the brand and quality, the average price is affordable for most of the population and much less than in many other countries (National Bureau of Statistics of China 2013). Moreover, studies suggest that people in China are virtually insensitive to the price of alcoholic beverages (Tian \& Liu 2011) and cigarettes (Lance et al. 2004), due to the fact that many alcoholic beverages and cigarettes are consumed at public expense and absence of accompanying control policies with tax or pricing increasing, and therefore raising prices might not reduce substance use as much as previously suggested.

\subsubsection{Regulating the availability}

Adolescents usually get alcohol and cigarettes from two types of sources: commercial sources (direct retail purchase), and social source (buying or being given from friends, relatives and acquaintances) (Ahmad \& Billimek 2007). Thus, another important way to restrict the substance use of adolescents is regulating 
the availability of alcoholic beverages and cigarettes since it can increase the economic and opportunity costs associated with obtaining alcohol and cigarette (Chaloupka et al. 2002). Approaches aimed at limiting the availability of alcohol and cigarettes include enacting partial or total bans, restricting hours and days of sale, and controlling the location, type, and number of retail outlets (Babor et al. 2010), as well as other means to decrease the physical availability of the substance.

Many countries have limited the availability of alcoholic beverages and cigarettes. For instance, strict control of the physical availability of alcohol and a comprehensive state monopoly on alcohol sales have been enacted and become more widespread and stringent in last two decades (Mäkela \& Österberg 2009). Meanwhile, limiting the availability of tobacco is one of the main activities enacted to reduce smoking in Finland. Since June 2007 smoking in restaurants has been banned and since April 2009 retail trade in tobacco products became subject to a licence at the outlet (Ministry of Social Affairs and Health of Finland 2010). Additionally, according to the new objectives of the Finnish Tobacco Act (Ministry of Social Affairs and Health of Finland 2010), in the future, there will be a total ban on the sale of snuff and tobacco products, or their trademarks may not be displayed in retail sale facilities. Contrarily, the Chinese government has taken much less action on regulating the availability of alcoholic beverages and cigarettes. Even through the tobacco products in China are manufactured by the national monopoly company, there is no strict limitation in place to restrict the time of sale for retail outlets, and no limitation of minimum age for legal purchase, which makes it easier for teenagers to obtain cigarettes. Similarly, despite the existence of a minimum legal age for the purchase alcohol, action to limit the availability of alcoholic beverages, on a national level, is absent in China. Alcohol is very common in China and it is available to the public in grocery stores and supermarkets.

\subsubsection{Restriction on marketing}

Previous studies have revealed consistent results confirming that exposure to the advertising of alcohol and cigarettes is strongly associated with young people's early onset, and the amount of alcohol use (Anderson et al. 2009; Ellickson et al. 2005; Jones \& Magee 2011), and smoking (Lovato et al. 2011; Tercyak et al. 2002). Hence, the restriction on advertising and other marketing methods of alcoholic beverages and cigarettes is crucial to protect young people from engaging in drinking and smoking.

Policies on marketing restriction on alcohol and tobacco are different among countries. The 1994 Finnish Alcohol Act legalised the advertising of alcoholic beverages with alcohol content from 1.2 to 22 per cent alcohol by volume. However, the law prohibits the advertising of such beverages if it is aimed at young people (Ministry of Social Affairs and Health of Finland 1997). Even at present, advertising alcoholic beverages is generally legal in China except for certain restrictions in light of the Chinese Advertisement Law 1996, for instance, advertisements are not allowed to feature children and/or adolescents. The 
WHO FCTC (WHO 2005) pointed out that every participating country shall undertake a comprehensive ban of all tobacco advertising, promotion and sponsorship. As one party of the WHO FCTC, sales promotion, and advertising of tobacco products are totally prohibited in Finland (Ministry of Social Affairs and Health of Finland 2010). However, in spite of the fact that China also joined the WHO FCTC in 2005 and published the Advertisement Law to ban direct tobacco advertising on movies, radio, television, newspaper and periodicals in 1994, tobacco advertising, indirectly, remains widespread (Li et al. 2009).

\subsubsection{Education and intervention}

Education and intervention strategies are the most common approaches to prevent and reduce alcohol- and tobacco-related problems. As Babor and colleagues (2010) discussed, education on alcohol use, on both individual- and population-level, usually aims to change: 1) knowledge about alcohol and risks related to drinking; 2) intentions to drink; 3) drinking behaviour; 4) frequency or seriousness of problems related to drinking; 5) public attitude to increase support for alcohol policies. These aims are also relevant for smoking.

For adolescents, the most comprehensive forms of education and interventions comprise school, family, and community programmes since they have positive effects on knowledge and attitude (Bangert-Drowns 1988). A considerable number of studies have shown that education programmes and interventions on substance use in school reduce the prevalence of alcohol use and smoking of schoolchildren (e.g. Botvin et al. 2003; Marsiglia et al. 2012). In spite of the fact that most of these studies have shown short-term decreases in substance use, systematic reviews of long-term follow-up school-based prevention revealed that there was little to no evidence of long-term effectiveness (Wiehe et al. 2005); even so, the implementation of national school-based education and interventions should still be addressed to prevent students' substance use, or at least to delay the onset of using. Additionally, school-level policies on restrictions of substance use among teachers are important since, in accordance with Social Cognitive Theory (Bandura 1986), students are more likely to use substances if they observe significant other's using those substances. Furthermore, family-based intervention on adolescent alcohol use and smoking was proved effective as a universal prevention programme for decreasing substance use of young people (Jackson \& Dickinson 2006; Loveland-Cherry et al. 1999). Individual-level education and intervention is also important for minimizing the risk of adolescent substance use. For instance, Tripodi and colleagues (2010) reviewed studies assessing the effectiveness of interventions reducing adolescent alcohol use, and found that individual-only interventions had a larger effect size than family-based interventions, and effect size decreased as length of follow-up increased.

Reducing alcohol use and smoking prevalence of adolescents are both priorities in public health for China and Finland, as well as other countries in the world. Various education and intervention programmes regarding decreasing adolescent substance use have been conducted in both countries (Chou et al. 
2006; Heikkinen et al. 2009; Pennanen 2012; Sarkola et al. 2011). In Finland, it should be noted that health education became a new independent school subject in basic and upper secondary schools in 2001, and was later adopted by The National Core Curriculum, which consists systematic education on alcohol use and smoking (Välimaa et al. 2008). In China, unlike Finland, although differential short- or long-term health education programmes have been carried out in schools, there is still an absence of a continuous and independent education subject on alcohol use and smoking, on a national scale. 


\section{SOCIOECONOMIC INEQUALITIES IN HEALTH AND HEALTH BEHAVIOUR}

The evidence of health inequalities has been accumulated from both social and medical research (Dalstra et al. 2006; Marmot 2005). Recently, burgeoning research on socioeconomic inequalities in adolescent health has received more and more attention. In the beginning of this chapter, background information of health inequalities and socioeconomic inequalities in health are described respectively. Explanations of the presence and the absence of socioeconomic difference in health are then discussed. Measurements of socioeconomic status in general and in adolescent populations are presented, followed by a detailed description of the Family Affluence Scale. Finally, the current situation of socioeconomic inequalities in adolescent health is reviewed with special focus on alcohol use and smoking.

\subsection{Definitions of health and social inequalities}

Health inequality is a term used to designate differences, variation, and disparities in the health achievements of individuals and groups (Kawachi et al. 2002). For instance, if there are higher incidence of disease(s) or health outcome(s) in group A as compared with group B of population C, then there is a presence of health inequality in that population. Woodward \& Kawachi (2000) documented that there were four arguments for reducing health inequalities: 1) Inequalities are unfair; 2) Inequalities affect everyone; 3) Inequalities are avoidable; and 4) Interventions to reduce health inequalities are cost effective.

In the UK, the Black Report of 1980 is noted as the definitive report that put health inequalities on the academic and policy agenda of industrialized countries (Department of Health and Social Security 1980). One year after the Black Report was published, the director General of the WHO at the time, Mahler (1981), defined and advocated 'Health for All', which means that health is to be brought within reach of everyone in a given country. Sadly, however, 
growth in health inequalities has been reported in recent years by various studies globally (Marmot 2005).

There are many dimensions along which health inequalities can be illustrated, such as gender, race, socioeconomic status, political power, culture assets, social assets, and human resources (Kawachi et al. 2002). Among those dimensions, inequalities in health among groups of different socioeconomic status or position are most frequently evaluated, since it constitutes one of the main challenges for public health (Marmot 2005). In light of the definition of health inequality mentioned earlier, if a group is divided in terms of socioeconomic status and if their health status varies accordingly, we can conclude that socioeconomic inequality in health exists in that population.

'Socioeconomic status (SES)', 'socioeconomic position (SEP)', and 'social class' are frequently used terms as the socioeconomic indicator in health inequality research. SES is a measure of one's access to collectively desired resources and is a fundamental construct in the social and health sciences (Oakes \& Rossi 2003). Since the last century, numerous studies have shown that socioeconomic inequalities in health were observed within and between countries (e.g. Mackenbach et al. 2008; Murray et al. 1998; WHO 2004b).

In general, the majority of research on SES and health revealed graded associations with lower SES usually related to poorer health or greater health-risk behaviour. For instance, studies have shown that age-adjusted risk of mortality and morbidity for those in the lowest socioeconomic group is higher than that for those in the highest group (Kunst et al. 1998; National Centre for Health Statistics 2008). However, research on socioeconomic inequalities in health indicates that poor health and health-risk behaviour are not simply confined to those at the bottom of the socioeconomic hierarchy. Thus, health varies according to all levels of SES, not only between those in poverty and those in well-off (Adler \& Ostrove 1999).

\subsection{Explanations of socioeconomic differences in health}

There are several explanations of socioeconomic differences in health. In 1982, four types of explanation were posed in Black Report (Townsend \& Davidson 1982), including artefact explanations, theories of natural or social selection, materialist explanations, and cultural/behavioural explanations. After the Black report, the causal models and selectional models have been developed as the two main explanations for socioeconomic differences in health. For instance, Adler and Ostrove (1999) stated that two alternative explanations can be attributed to the association of SES and health, of which one is that SES affects health (social causation) (Mackenbach et al. 1994), and the other being that health influences socioeconomic status (social drift or health selection) (Stronks 1997). Though the influence of SES and health is mutual; research findings are more compelling for social causation than for social drift (Adler \& Ostrove 1999). These explanations of socioeconomic inequalities in health and the differ- 
ent pathways by which SES influences health related to adolescent are briefly described as follows.

\subsubsection{Artefact explanations}

In the first explanation of the relationship between SES and health, both variables are considered as artefacts of the measurement process and therefore their observed relationship may itself be an artefact of little casual significance (Department of Health and Social Security 1980). In other words, it means that the size of the observed SES difference in health depends on the measurement of both class and health (Macintyre \& Hunt 1997). This explanation is crucially important concerning research on SES inequalities in adolescent health, since their SES is mostly derived from their parent's SES. Therefore, if the measurement of the parents' SES is not appropriate, the adolescent's SES is not valid either, and therefore the association of the SES and health will be artefact. Gecková (2002) pointed out that it is better to examine socioeconomic difference in adolescent health through different measures of SES and health.

\subsubsection{Health selection}

In the health selection model, SES is relegated as a dependent variable while health has the greater degree of causal significance. This explanation addresses the influence of health on socioeconomic mobility. Health selection is considered as a process of sorting or allocation of individuals with different health potential through the educational and occupational systems into an achieved social class (West 1991). The simple version of this model can be described as that health can influence the achieved class position (Macintyre 1997), or that healthier youngsters experience upwards social mobility and less healthy ones achieve less mobility (West 1990). Stronks (1997) also suggested that the health selection may proceed through occupation during adulthood (intragenerational mobility) and educational achievement during adolescence (intergenerational mobility). Although the effects of selection should be particularly marked in the period between entry into labour market and the achievement of a stable social class, it is not only limited to that period. For instance, it has been found that selection by health-related lifestyle into educational tracks in adolescents is an important mechanism creating socioeconomic differences in health in adulthood (Koivusilta 2000).

\subsubsection{Social causation}

The other explanation of the socioeconomic difference in health is social causation, which emphasises the role of SES in the distribution of health and wellbeing (Department of Health and Social Security 1980). Evidence has indicated that, in general, health risk behaviour or ill-health is more common among people in lower social class. There are three mainly used point of views in health inequality research in terms of this model (Macintyre 1987): life circumstance; 
life style; and knowledge, attitude and values. Firstly, life circumstances referring to the material conditions may influence health status and is not equally distributed among social groups (Macintyre 1986). People in different occupational and social positions are likely to differ in the extent to which they experience life events and circumstances of living and working, which may influence their health. To reduce the socioeconomic inequalities in health, the life circumstances should be improved according to this explanation (Stronks 1997). Secondly, the personal health behaviours and health-related lifestyles vary by SES due to variability in accessibility, affordability, and custom of culture, which ultimately results in differences in their health (Smith et al. 1990). In terms of this explanation, interventions with concerns on individuals' behaviour should be encouraged in order to change their life styles and therefore reduce health inequalities. Finally, it has been suggested that knowledge, attitudes and values may contribute to socioeconomic differences in health directly by impacting on health behaviour. For example, coping strategies, locus of control, learned helplessness may influence how young people live and respond to their lives and environments. The effect can be also indirectly, like health knowledge, by shaping behaviours which are more indirect causes of health (Macintyre 1986).

\subsubsection{Different exposure and different vulnerability}

Based on the social causation theory, there are two hypotheses, one is the different exposure and the other is the different vulnerability (Gecková 2002). In terms of the hypothesis of different exposure, socioeconomic inequalities in health may be interpreted by the different occurrence of health determinants. In comparison with people in a higher SES group, the determinants of negative health effects, like alcohol use and smoking, occur more frequently, and determinants of positive health effects, like physical activity and nutrition, occur less frequently among people in a lower SES group. Alternatively, according to the hypothesis of the different vulnerability, people from the higher SES groups have more ability to cope with determinants of health while the people from the lower SES groups are more vulnerable to these health determinants. However, it is questioned whether these two hypotheses can interpret the unequal distribution of health in the population (Stronks 1997; Tuinstra et al. 1998).

\subsection{Measurement of socioeconomic status}

Kawachi and colleagues (2002) have summarized that there are two different approaches to evaluating health inequalities: measuring social group differences in health which is most commonly used, and measuring the distribution of health status across individuals in a population. No matter what approach is used, to understanding the health disparities associated with SES, it is essential to have reliable and valid measures in order to get accurate information on SES. For an adult population, SES is traditionally assessed by either univariate 
measures, such as education attainment, occupation status, income and family income, wealth, poverty, neighbourhood and area/contextual level measures (Galobardes et al. 2006a; Ostrove \& Adler 1998; Shrewsbury \& Wardle 2008), or composite measures, including measures of material and social deprivation, like Mayer-Jencks' Material Hardship measure and Townsend Index, and measures of social standing and prestige, like the Duncan Socioeconomic Index and the Hollingshead Index of Social Prestige or Position (Galabardes et al. 2006b; Shavers 2007). Although these measures have been validated for assessing SES, the fact that there are various measures as well as a variety of overlapping concepts, indicate the complexity of the construct of SES. It has been pointed out that there is no single best indicator of SES suitable for all study aims and applicable at all-time points in all settings (Galobardes et al. 2006b). Moreover, Braveman and colleagues (2005) have argued that in health research, regardless of the measure(s) used, most studies use SES variables without justifying why they have been selected over others.

\subsubsection{Measurement of adolescents' SES}

With regard to the measurement of SES among adolescents, the situation becomes more complex. It is problematic if SES indicators for an adult population are used to measure adolescents' SES. First of all, adolescence, normally is in the period spent mostly studying in school and not legally allowed to work, so they do not have any, or have very little economic resources themselves, resulting in the absence of information on income. Secondly, the majority of adolescents are in school and usually have not finished their studies yet. Therefore, it is not surprising that when investigating adolescents in school, the inquiry of education attainment may also lead to exactly the same results since they are in the same educational level.

Accordingly, adolescents' SES is usually measured by using the information regarding their parents' SES, such as parents' education and occupation, and household income. However, one should be aware of the difficulties of measuring adolescents' SES when using their parental SES as a proxy. Currie and her colleagues (2008b) argued that it is still uncertain whether parents' SES should be used as a proxy. For instance, the adolescents' SES are not always reflecting their parental SES, and even if it does so, which is the most relevant aspect of SES? Another issue related to adolescents' SES measuring is the association between objective SEP and subjective SES. Karvonen and Rahkonen (2011) found that the subjective assessment of SES contributes to health inequalities in young people largely independent of objective SEP. Furthermore, methodologically there are also difficulties in obtaining accurate information regarding parental SES from adolescents due to the lack of knowledge or unwillingness to reveal the relevant information (Currie et al. 2008b), which results in the low completion or high non-response rates when collecting data of adolescents self-reported parental SES (Molcho et al. 2007; Wardle et al. 2002). It has also been reported that there is a higher non-response rate for those adolescents in the low SES group (Wardle et al. 2004). Therefore, when measuring the SES of 
adolescents, except for indicators reflecting parents' SES, it is crucially important to formulate accurate and effective indicators, which are easy for adolescents to answer and minimize the potential bias in a certain subgroup.

\subsubsection{Family Affluence Scale (FAS)}

Among the subjective indicators used for measuring adolescents' SES, the HBSC Family Affluence Scale (FAS) is a measure which has recently been developed and widely used to address the association of SES and health outcomes in both domestically and internationally comparative studies (Currie et al. 2008b; Maes et al. 2006; Pickett et al. 2005; Torsheim et al. 2004, 2006).

Magklara and colleagues (2010) argued that socioeconomic inequalities in adolescent health are more easily demonstrated with more subjective socioeconomic indicators. The FAS used in the HBSC survey contains several items which assess the SES of the adolescent based on the material condition of their household, which was originally based on the work of Carstairs and Morris (1991) and Townsend (1987). In the 1997/1998 HBSC survey, three FAS items were employed, which asked about family car ownership, the child's use of their own bedroom, and the number of family holidays; this is referred to as the first version of FAS (FAS I) (Currie et al. 2004). Later, in the 2001/2002 HBSC survey, an item on number of family computers was added and these four FAS items were collectively named as the second version of FAS (FAS II) (Currie et al. 2004).

The FAS II has been used extensively in the HBSC study in the past decade to examine and describe socioeconomic inequalities in relation to adolescent health outcomes. Compared to the traditional SES indicators, one advantage of the FAS II items is that they can greatly reduce the non-response rate. It has been reported that the FAS II items showed a higher completion rate than items on parental education and occupation (Boudreau \& Poulin 2009; Lin 2011; Molcho et al. 2007). Validation studies of the FAS II have been done in many HBSC member countries as well as some non-HBSC member countries, from which the results showed that the FAS II items have a moderate internal reliability (Lin 2011; Molcho et al. 2007; Wardle et al. 2002), a significant association with parental education and occupation (Currie et al. 1997; Molcho et al. 2007), a high agreement rate between adolescents and parents (Andersen et al. 2008), and a satisfactory correlation with the national wealth indicator, Gross Domestic Product (GDP) (Boyce et al. 2006). Although the test-retest reliability of FAS II items has not been examined, the rather higher agreement can be predicted due to the objectivity and stability of the indicators (Currie et al. 2008b). Moreover, Schnohr and her colleagues (2008) have analysed the differential item functioning (DIF) of FAS II and demonstrated that it can be used as an interval scale and a measure of wealth within a country and between countries.

The FAS II has also recently been used as a SES indicator outside the context of the HBSC study (e.g. West \& Sweeting 2004) or in countries which are not a member of the HBSC network (e.g. Cho \& Khang 2010). Because every country has its own culture and economic status, the FAS II should be used af- 
ter examining the applicability and validity in a certain context. However, since the HBSC study is limited at the moment to countries in Europe and North America, very few studies assessing the use of the FAS II have been done elsewhere in the world. There are only two studies which have been done recently to assess the use of FAS II in South Korea (Cho \& Khang 2010) and Taiwan (Lin 2011), and no similar study has been done in mainland China.

\subsection{Studies of socioeconomic inequalities in adolescent with focus on alcohol use and smoking}

\subsubsection{Current status of studies}

Most existing studies concerning social inequalities in health and health behaviour pay attention to the adult population. Studies focussing on socioeconomic inequalities in adolescent health and health behaviour are less common (DiLiberti 2000; Marmot 2005). It has been well documented that low socioeconomic status has been consistently related to poor health outcomes among young children and adults (Walker et al. 2007; West et al. 2004). However, research on associations between socioeconomic status (SES) and adolescents' health outcomes revealed inconsistent results in many essential aspects of health (Starfield et al. 2002; Torsheim et al. 2004; West et al. 2004). A review done by Starfield et al. (2002) revealed the existence of socioeconomic difference in several aspects of health among adolescents, but not in all.

Similar to the health outcomes, studies conducted among adults and children have shown that unhealthy behaviours are more prevalent in lower socioeconomic groups, and contribute to socioeconomic inequalities in health (Laaksonen et al. 2008; van Oort et al. 2005; Woodward et al. 2003). However, there has been debate regarding adolescents, since in contrast to adults and children, most studies have found little or inconsistent associations between SES and health behaviours among adolescents. A review by Hanson \& Chen (2007) demonstrated that different associations can be found between SES and unhealthy behaviours in adolescence, such as more traditional associations (low SES is related to greater negative behaviours), reversed associations (high SES is related to greater negative health behaviours), and null/weak associations (non-significant relation between SES and health behaviours).

The three possible explanations for the divergent findings concerning the relationship between SES and health among adolescent can be summarized as: 1) the different indicators of SES used in research; 2) not including youthspecific indicators of social class: and 3) most studies being done in high-income countries (Ritterman et al. 2009). The hypothesis of latent differences and the hypothesis of the buffer effect might be the two possible explanations for the absence of socioeconomic inequalities in health (Gecková 2002). The hypothesis of latent differences considers that although the socioeconomic health differ- 
ences in adolescents emerge, they still cannot be measured by the current indicators of health outcomes. The hypothesis of buffer effect is that social circumstances young people live in, such as family support, peer culture, and school setting, can comprise a buffer for adolescents against the health-risk behaviour and unhealthy lifestyle.

These above-mentioned facts suggest that the socioeconomic inequality in health and health behaviour is age-specific (West et al. 2004). Gecková (2002) has described five reasons for studying socioeconomic inequalities in adolescent health and health behaviour: 1) health from childhood and adolescence can extend in adulthood and contribute to health inequalities in later life; 2) many patterns of health behaviour are formulated in adolescence and therefore they can influence the future adult health; 3 ) Socioeconomic inequalities in health are age specific; 4) adolescence is an important period with regard to the final adult stratification; and 5) adolescence provides the opportunities for reducing health inequalities since interventions are more effective if they are implemented earlier in life.

\subsubsection{Socioeconomic inequalities in adolescent alcohol use}

Several studies have investigated the presence of social inequalities of alcohol use (Bloomfield et al. 2006; Kunst et al. 1996), and the clear and persistent social gradient in alcohol-related mortality among adults has been documented by former studies (Harrison \& Gardiner 1999; Mäkelä et al. 2003). Most of studies, however, focused only on the adult population and there is not enough research targeting alcohol use of adolescents. In addition, the evidence based on previous studies about the relationship between socioeconomic status and adolescent drinking behaviour is inconsistent and even contradictory. For instance, some studies revealed more frequent adolescent drinking behaviour among lower socioeconomic groups (Melotti et al. 2011), while others reported that there is no, only limited, or even an inverse social gradient in adolescent alcohol use (Richter et al. 2006; Vereecken et al. 2003a).

For Finnish adolescents, socioeconomic background was found to be a strong determinant of weekly alcohol use that young people from upper whiter-collar employees' families were more likely drinking than those from the lower SES groups from AHLS (Karvonen \& Rimpelä 1996), whereas no association was found between family affluence and weekly drinking from Finnish HBSC study (Currie et al. 2008a). Lintonen and his colleagues (2000) found that monthly drinking among 14-year-old girls was associated with their guardian's education that the proportion of monthly drinkers among daughters of parents with no more than a comprehensive or secondary education was one and a half time greater than those girls of parents with a higher education and there were no difference among boys. However, data from the ESPAD survey suggested that guardian's education also has an effect on drinking among boys (Ahlström et al. 2004). For Chinese adolescents, the studies on socioeconomic differences in adolescents' alcohol use were reported rather less. A Youth Risk Behaviour Survey done in Guangzhou showed that higher parental education was posi- 
tively associated with adolescent current and lifetime alcohol use (Wang et al. 2009).

\subsubsection{Socioeconomic inequalities in adolescent smoking}

Similar to the alcohol research, numerous studies regarding socioeconomic inequalities and smoking have been done in adult populations and the results indicate that people from lower SES groups were more likely to smoke (Adams et al. 2009; Jha et al. 2006; Machenbach et al. 2008; Mistry et al. 2011; Siahpush et al. 2006). In contrast to studies on adult populations, the literature addressing the socioeconomic inequalities in smoking among adolescents is limited. The literature revealed inconsistent and even contradictory results. Several studies showed that there is an association between smoking and SES during adolescence. For instance, Gecková and her colleagues (2005) found that Slovakian adolescents' smoking was indirectly influenced by SES via the influence of parents' and peers' smoking behaviour. Social inequalities associated with daily smoking among adolescents were also found in several European countries (Schnohr et al. 2009), while research conducted in Netherlands (Tuinstra et al. 1998) and Croatia (Simetin et al. 2010) indicated that there was no relationship between SES and adolescents' smoking. Moreover, a study analysing trends in socioeconomic differences and smoking among German schoolchildren, found that family affluence only had a weak effect on regular smoking (Richter \& Leppin 2007).

These inconsistent results were also observed in studies concerning Chinese and Finnish adolescents. For instance, based on the HBSC data, Schnoher and colleagues (2009) found that Finnish adolescents from the low affluent families were more likely to be daily smokers and the results from ESPAD survey showed a minor but persistent difference in smoking among adolescents by parent education that more smokers among young people whose parents are with no more than comprehensive schooling than among young people whose parents have an academic education (Ahlström et al. 2004), while Paavola et al. (2004) demonstrated that there was no relationship between SES and adolescents' smoking. In studies concerning Chinese adolescents, positive associations between daily smoking and SES were not found in Guangzhou Youth Risk Behaviour Survey (Wang et al. 2009), but were found in the China Adolescent Behavioural Risk Survey (Ji 2007).

To summarize, studies on socioeconomic differences in alcohol use and smoking among adolescents provided inconsistent evidence in different countries/regions, or even within the same country/region. Moreover, such studies have been mainly contacted in North American and European countries and there is still a lack in examining the patterns of socioeconomic position and adolescents' alcohol use and smoking in Chinese context. 


\section{CROSS-NATIONAL COMPARATIVE RESEARCH ON ADOLESCENT HEALTH BEHAVIOUR}

As an indicator of globalization, more and more cross-national comparative studies on adolescent health behaviour have been and are being undertaken. In this chapter, a brief review of the general information on cross-national comparative research will be given followed by a short illustration of the HBSC study, as one example of those cross-national studies on adolescent health behaviour, which is used in present study. The rationale and significance of applying the HBSC study in China are then described.

\subsection{Cross-national comparative research}

Hantrais \& Mangen (1996) mentioned that a study can be simply defined as a cross-national comparative research if one or more units in two or more countries are compared regarding the same concepts and systematic analysis, usually with the intention of explaining them and generalising from them. A broad definition of comparative health research, described by Øvretveit (1998), is research that creates empirical or explanatory knowledge about all aspects of health, by making comparisons using scientific methods that are appropriate for the subject studied and for the purpose of the research. Thus, it can be concluded that cross-national comparative research on adolescent health behaviour is that using the right approach and appropriate research methods to compare adolescent health behaviour in two or more countries.

\subsubsection{Purpose and significance}

Øvretveit (1998) has summarised the aim of comparative research on health is one or more of the following: 1) to discover whether an event, phenomena, or entity that occurs in one place also occurs in another; 2 ) to discover significant similarities or differences between comparable items in different areas; 3 ) to 
measure the size difference or gather data about the meaning of the items or phenomena in different areas; 4) to understand and explain the similarities or differences between items in different places; 5) to contribute to decisions about actions to improve health; 6 ) to contribute to the adoption of improved practices or procedures by comparing a new more effective procedure to one customarily used elsewhere. The purpose of cross-national comparative research on adolescent health behaviour is covered by the afore-mentioned aims, and the majority of them are to explore or explain the similarities and/or differences of adolescent health behaviour in different countries.

Why should we do cross-national comparative research and what are the benefits of this type research? Those two questions concerning the rationale and significance of undertaking cross-national comparative research should always be considered beforehand. First of all, cross-national comparative research can lead to fresh insights and a deeper understanding of issues in different countries (Hantrais \& Mangen 1996). Regarding the studies on health behaviour of adolescents, comparative study between countries, for example, Finland and Switzerland (Karvonen et al. 2000), or cities in different countries, for example, Helsinki and Glasgow (Karvonen et al. 2001), can provide information on whether there were cross-cultural patterns of health behaviours among young people. Secondly, it may force the researchers to understand the thought process of another culture, while also reconsidering their own country from the perspective of a skilled observer from outside, which in itself might be seen as a sufficient justification for conducting cross-national research (Lisle 1985). Moreover, the benefits are considerable not only in the scientific findings from the research, but also in establishing valuable collaboration between countries. When researchers with different backgrounds from different countries are brought together on collaborative cross-national projects, their experience and knowledge of different intellectual traditions will be mutually beneficial.

\subsubsection{Limitations and challenges}

There are some limitations and challenges concerning cross-national comparative research due to the nature of this type of research. Among those limitations and challenges, especially for research through self-reported measures, question design and translation are two of the most important issues since questions are perceived in context and the different contexts in which translated questions are processed (different countries and cultures) may change the perceived meaning of the question (Harkness 2003). Rosen (1987) also pointed out that there are unreliable data-gathering methods and difficulties standardising the datagathering methods between countries. Despite of those methodological limitations, practical challenges regarding undertaking cross-national comparative research may not be ignored, such as securing finance and resources for the research, organising and managing a project, and different priority given to the research by different host countries (Øvretveit 1998). 


\subsection{The Health Behaviour in School-aged Children (HBSC) study}

There are many cross-sectional or longitudinal surveys in the world with their foci on adolescents' health and health behaviour on both national and international levels. These studies either investigate the health and health behaviour comprehensively, such as the Health Behaviour in School-aged Children (HBSC), a WHO collaborative cross-national survey (Currie et al. 2009), or just target a specific health behaviour/outcome or a group of health behaviours/outcomes, such as the WHO Global Youth Tobacco Survey (GYTS) (Warren et al. 2000), the Youth Risk Behaviour Surveillance System (YRBSS) (Brener et al. 2004), the European School Survey Project on Alcohol and Other Drugs (ESPAD) (Plant \& Miller 2001).

Among those abovementioned cross-national studies on adolescent health behaviour, the Health Behaviour in School-aged Children (HBSC) study is one of the very first large-scale international surveys on adolescent health and health behaviour (Roberts et al. 2007). McQueen (2009) summarized the three salient characteristics that have defined the HBSC study compared to other studies: 1) it has sustained over a long period of time (nearly 30 years); 2) it has built international research protocol for surveys in many and varied countries over that time; 3 ) it is driven by an underlying theoretical approach that is the leading issues of the time.

\subsubsection{Research methods}

The research methods of the HBSC study is one of the most challenging components of the HBSC study due to the complex nature of exploring young people's health and health behaviour in many countries, with different structural and practical backgrounds and situations, such as school systems and language (Aarø et al. 1986; Roberts et al. 2009; Smith et al. 1992). In order to collect reliable, valid and comparable data among the HBSC survey countries, a standardised international research protocol followed by all members is indispensable. The research protocol is revised and updated for the forthcoming survey every four years, and the current one is for the 2009/10 study (Griebler et al. 2010). In the international research protocol, conceptual framework, scientific rationales, questionnaire content, survey methods and instructions of the HBSC study are defined as well as the rules regarding the international data application and publishing (Roberts et al. 2009).

According to the conceptual framework of the HBSC study, the survey is conducted every four years based on a self-reported questionnaire comprising a range of health indicators (e.g. self-rated health, life satisfaction, health complaints, body image, injuries, obesity), and health behaviours (e.g. physical activity, sedentary behaviour, eating and dieting, oral health, substance use, sexual behaviour, fighting and bullying) of adolescents and their life circumstances (e.g. family, peer, and school). Studies addressing the validation of the survey items have been done at national and international level, and as a result scien- 
tific articles or reports were published along with the development of the HBSC study (Booth et al. 2001; Boyce et al. 2006; Elgar et al. 2005; Haugland \& Wold 2001; Ojala et al. 2005; Rangul et al. 2008; Ravens-Sieberer et al. 2008; Torsheim et al. 1997, 2000; Vereecken et al. 2003b; Tynjälä 1999; Vuori et al. 2005). Another challenging issue concerning the survey questionnaire used in a number of countries is language, both for culturally relevant and comprehensible reasons (Sperber et al. 1994). The international research protocol is written in English, on which all core and optional survey items are listed. To avoid any possible inappropriate or inaccurate translation of the questionnaire, prior to the survey, the HBSC research team of each member country, in which English is not used as the official language, translate the questions into its own mother tongue and the questions are then translated back into English by a third party so that they can be compared against the original at the International Coordinating Centre for the HBSC. This process contributes not only to minimize the errors of language translation (Roberts et al. 2007), but also to ensure are the students understand the questions (Roberts et al. 2009).

The target population of the HBSC study is schoolchildren aged 11, 13 and 15 , which represents the onset of adolescence, the challenge of physical and emotional changes, and the middle of age when important life and career decisions are beginning to be made respectively. The recommendation on the sample size is about 1500 students for each group, and each country may decide the sampling procedure and strategy to ensure the maximal representation of the data.

\subsubsection{HBSC in non-member countries}

Under the coordinating of the WHO Regional Office for Europe, the current 43 HBSC member countries are restricted within Europe (41 countries) and North America (Unite States and Canada). However, for the purpose of understanding young people's health in a wider spectrum, several studies have been carried out previously using the HBSC survey method in non-member countries with the cooperation between the HBSC research team from a member country and researchers from a local institute of the non-member country. For example, the HBSC study was carried out in Semarang, Indonesia (Smet et al. 1999), the Pacific Islands (Phongsavan et al. 2005), Cabo Verde (Gonçalves et al. 2005) and also more recently in Taiwan (Lin et al. 2011).

In order to make a clear criterion of the status of membership, the HBSC has recently redefined its membership into three categories: full member, satellite member and linked project collaboration member. For applying for full membership, countries should be in the WHO European Region which is currently not part of the HBSC study. The satellite membership refers to those countries/regions which are geographically within Europe but not recognised by the WHO or EU as official member states. The Linked Project Status is created by the HBSC Network in order gain further understanding of those countries who use or are interested in using HBSC instruments outside of Europe and North America. Any eligible research team from a non-HBSC member 
country/region can apply to be a linked project to the HBSC International Coordinating Centre through submitting the registration form. After approval by the HBSC International Coordinating Centre, the linked project country/region can conduct a national/regional study using the HBSC research protocol, as well as be invited to any open non-member HBSC conferences in terms of the agreed regulation. Currently, the HBSC Linked Project officially comprises research teams from China Institute of Sport Science (CISS), Kosovo, Kuwait University, Lebanese University, and Taipei University. Each linked project research team has contacts from the research team of a HBSC member country. The HBSC Linked Project provides a valuable opportunity for countries all over the world to explore young people's health and health behaviour using the same HBSC instrument and therefore to make cross-national comparison.

\subsection{The application of the HBSC study in China}

\subsubsection{Current status of health behaviour of adolescents in China}

The health behaviour of the youth is related to their future health and life style. From the global point of view, the situation of health and health behaviour of young people in China cannot be ignored since, as the most populous and the biggest developing country in the world, there are nearly 400 million young people living there. Chinese economic development has been witnessed in the preceding three decades and the standards of living and health care systems for the Chinese people have been improved in many aspects (Le Deu et al. 2012; Zhang et al. 2010). As a result, the life expectancy has been increasing and the child mortality has been decreasing in China (WHO 2012b).

However, with regard to the health and health behaviour of Chinese young people, the Chinese society is still facing many problems as many other countries in the world. Rapid economic development in China has brought not only the benefit of improved young people's health and living conditions, but also certain negative effects. For instance, recent studies indicate that with changes in lifestyle such as increased energy intake and decreased physical activity among 367 million young people in China age from 6 to 17 years old, there is a lack of physical activity (Du et al. 2002) and therefore physical fitness tends to drop year on year as well as overweight and obesity being an increasing trend (General Sport Administration of China 2006, 2011). In the meantime, substance use, like tobacco smoking, alcohol and use of psychoactive substances has been becoming more and more frequent among Chinese adolescents (Liu et al. 2001; Page et al. 2011; Wang et al. 2009; Xing et al. 2002; Zhang \& Cai 2003). These facts concerning the health behaviour of Chinese adolescents are not optimistic and actions should be taken to promote health enhancing behaviours and reduce health risk behaviours. 


\subsubsection{Rationale and significance of HBSC study in China}

It is important to understand the prevalence and trends of health behaviour among young people and the factors influencing those health behaviours in order to design more effective interventions promoting their health. The first step toward understanding young people's health is to obtain abundant and accurate data which represent the prevalence of health behaviours of young people. To achieve this goal, a number of surveys have been conducted in China, with or without foreign partners, on an international or national level.

In 1999, the Global Youth Tobacco Survey (GYTS), an international surveillance developed by the World Health Organization (WHO) and the U.S. Centres for Disease Control and Prevention (CDC), with the specific aim of targeting smoking of adolescents aged 13-15 years, was conducted in four cities in China (Chongqing, Guangzhou, Shanghai, Tianjin) (Warren et al. 2000). In addition, the WHO has initiated, in collaboration with the U.S. CDC, the Global School-based Health Surveillance System (GSHS) in a number of developing countries, of which the aim is to assess and measure a range of health behaviours and protective factors (e.g. alcohol and tobacco use, dietary behaviours, drug use, hygiene, mental health, physical activity, protective factors, sexual behaviours, violence and unintentional injury) (Brown et al. 2009) among students aged 13-15 years. In 2003, as one of the participation countries of GSHS, surveys were carried out in four cities in China (Beijing, Hangzhou, Wuhan, and Urumqi). Meanwhile, another study, the Youth Risk Behaviour Surveillance System (YRBSS), which was developed by U.S. CDC, with its focuses on risk behaviours, has been conducted similarly in Beijing (Cheng et al. 2003) and Shanghai (Luo et al. 2003; Peng et al. 2003). The international collaborative studies provide a platform to investigate the health behaviour of Chinese adolescents and make it feasible to compare the results with other nations. However, those studies usually focus only on a certain type of behaviour or do not pay much attention to the social context in which young people live.

Additionally to those aforementioned international collaborative studies, various surveys on the health behaviour of Chinese adolescents have also been conducted independently by Chinese researchers. However the common characteristics of most of those studies can be summarized as they are crosssectional studies conducted in one or several cities/regions with regard to a particular, or a cluster of health behaviours of adolescents from rather small sample sizes. Furthermore, these results cannot be utilized in the cross-national comparison with studies in other countries, or even cannot be compared with other studies in China due to the different survey methods and procedures employed. Nevertheless, there are very few studies which can give a comprehensive and comparable portfolio of health behaviours of Chinese young people and therefore address the importance of health behaviour of adolescents. Therefore, more research, especially international collaborative studies, should be encouraged. 
Under such circumstances as described above, the application of the HBSC study in China is significant and worthwhile. The HBSC study comprehensively explores young people's health and health behaviour in their social context, which has been lacking in previous Chinese studies and crucial for understanding Chinese adolescents' health behaviour and to therefore design and implement health policies and interventions. The HBSC international research protocol allows the findings from China to be compared to other member countries which gives a clearer indication of what the situation of the Chinese schoolaged children's behaviour is compared with other countries. The results of the study also provide a chance to examine the health policy and young people's health as influenced by different circumstances in western and eastern countries. The study is also an opportunity to share ideas and experience of health education and health promotion both in academic and practical ways with China.

Additionally, the HBSC study in China can contribute to the HBSC study itself. The current participating countries of the HBSC are only within Europe and North America, and since the HBSC study is a tool to examine health behaviour of young people, it is important to seek more international support to examine whether the survey instrument is useful in different continents and cultures. Therefore, for the development of the application of the HBSC study, it is significant and meaningful to expand its boarders in the future to include China, which has the largest population of school-aged children in the world. 


\section{AIMS OF THE STUDY}

The purpose of this study was two-fold. First, the study examined the feasibility of implementing the HBSC survey, and the reliability of selected items related to health behaviour in the survey questionnaire in a sample of schoolchildren in Beijing, China. The first part of the study also investigated the completion rate of the traditional indicators measuring SES, compared with the FAS II, using the indicators of SES as measures in the HBSC study as well as in the later socioeconomic inequality analyses in the present study, and then examined the reliability and validity of the FAS II items in a Chinese adolescent population. Second, the study explored the associations between the socioeconomic status of adolescents and adolescents' alcohol use and smoking in China and Finland, and then compared the similarities and differences in these two countries. The specific research questions for the study were as follows:

1. What is the feasibility of implementing the HBSC survey, and what is the test-retest reliability of selected survey items related to health behaviour in Beijing, China? (Study I)

2. What are the reliability and validity of the FAS II items in a Chinese adolescent population? (Study II)

3. What are the associations between socioeconomic status and alcohol use of adolescents in China and Finland and does it differ in two countries? (Study III)

4. What are the associations between socioeconomic status and smoking of adolescents in China and Finland and does it differ in two countries? (Study IV) 


\section{METHODS}

The data used in this study were based on the Health Behaviour in School-aged Children (HBSC), a World Health Organization collaborative cross-national survey. The main aim of the HBSC study is to understand adolescent health related behaviours in their social context, where they live, at school, with family and friends (Currie et al. 2009). The HBSC survey is conducted among school children every four years and follows a standard international research protocol (Robert et al. 2007). As one of the initial countries of the HBSC study, Finland has carried out all surveys since 1984. In spite of the HBSC member countries being restricted to within Europe and North America, the Chinese HBSC linked project made it possible to conduct the HBSC survey following by the identical research protocol in China.

This study comprised three parts of data which were obtained from the pilot study for the Chinese HBSC linked project (test-retest reliability study in Beijing, China 2008) (I \& part of II), the Chinese HBSC linked project survey in 2008 (part of II, III, \& IV) and the Finnish HBSC survey 2006 (III \& IV). The detailed information of these surveys is described in the remainder of this chapter.

\subsection{Sampling location}

The data used in this study were collected from Beijing, China, and Finland. Beijing is the capital of China, consisting of 16 districts and 2 counties, with the total population 16.95 million by the end of 2009. Beijing has 653,255 students in 1104 primary schools (students aged from 6 to 12 years) and 508,327 students in 634 secondary and upper secondary schools (students aged from 13 to 18 years) (Beijing Municipal Bureau of Statistics 2010). In 2010, the average annual income in Beijing was 29073 Chinese Yuan (around 3,274€) per person, which is approximately 1.2 times the average annual income for China $(2,692 €)$ (Beijing Municipal Bureau of Statistics 2010). Due to the limited funding and resources, the China HBSC linked project was only conducted in the Beijing area at the 
current stage. Although schoolchildren from Beijing cannot represent the whole of China, the data can be still used to illustrate the phenomenon that the present study focuses on as one regional reflection of China.

Finland is a Nordic country with the total pupation 5.37 million by the end of 2010 (Statistics Finland, 2011). According to Statistics Finland (2012), there were 2789 comprehensive schools in Finland (students aged from 7 to 16 years), with 539,545 pupils in 2012. In 2011, the average annual income in Finland was 26,555€ per person (Statistics Finland 2013). In light of The World Bank's (2010) classifications of countries regarding income, China belongs to the uppermiddle-income economies, representing a developing country, and Finland belongs to the high high-income economies, representing as a developed country.

\subsection{Sampling procedure}

The target population for the HBSC survey is young people attending school aged 11, 13 and 15, representing the onset of adolescence, the challenge of physical and emotional changes, and the middle years when important life and carrier decisions are beginning to be made (Roberts et al. 2009). The desired mean age for the three age groups is 11.5, 13.5 and 15.5 years and according to the recommendation by the HBSC research protocol, sample size for each of the three groups is about 1500 students to meet the requirement of a $95 \%$ confidence interval of $+/-3 \%$ around a proportion of $50 \%$ and a design factor of 1.2 (Currie et al. 2001). Countries participating in the HBSC survey may choose to stratify their samples to make sure of the proper representation by geography, ethnic group, school type and so on. Cluster sampling is used and the basic sampling unit is school or school class (Roberts et al. 2009). The detailed information regarding the sampling procedure of this study is described as follows.

\subsubsection{Test-retest reliability study in Beijing, China 2008}

For the analyses of test-retest reliability of selected HBSC survey questionnaire items in Beijing, China (I \& part of II), two classes in grade 6 (students aged around 11 years old) and two classes in grade 10 (students aged around 15 years old) were randomly drawn from the two conventional sample schools. All the students $(\mathrm{N}=139)$ in these four classes participated in the test. Of those respondents, all the students from one class in grade 6 and two classes in grade 10 completed the questionnaire in the retest. Students from one class in grade 6 did not participate in the retest due to the overlap of the school schedule and the retest. No significant difference of the characteristics was found in the test between the class in grade 6, of which students participated in both the test and retest $(n=44)$, and the drop out class $(n=44)$. The response rates of the test and retest for the students in the participating classes were $100 \%$. The final sample for the test-retest study therefore consisted of 95 students aged 11 and 15 years (Table 1). The proportion of boys and girls was almost equal in the younger age 
group, but among the older age group, there were more boys than girls. The mean age of respondents did not differ between boys and girls in either age group.

TABLE 1 Demographic characteristics of respondents of test-retest reliability study in Beijing, China 2008.

\begin{tabular}{|c|c|c|c|c|c|c|c|c|c|c|c|c|}
\hline & \multicolumn{4}{|c|}{ Test $(n=139)$} & \multicolumn{4}{|c|}{ Retest $(\mathrm{n}=95)$} & \multicolumn{4}{|c|}{$\begin{array}{l}\text { Age of respondents in both } \\
\text { tests }(\mathrm{n}=95)\end{array}$} \\
\hline & \multicolumn{2}{|l|}{11} & \multicolumn{2}{|l|}{15} & \multicolumn{2}{|l|}{11} & \multicolumn{2}{|l|}{15} & \multicolumn{2}{|l|}{11} & \multicolumn{2}{|l|}{15} \\
\hline & $\mathrm{n}$ & $\%$ & $\mathrm{n}$ & $\%$ & $\mathrm{n}$ & $\%$ & $\mathrm{n}$ & $\%$ & Mean & SD & Mean & SD \\
\hline Boys & 41 & 46.6 & 31 & 60.8 & 20 & 45.5 & 31 & 60.8 & 11.79 & 0.30 & 15.81 & 0.34 \\
\hline Girls & 47 & 53.4 & 20 & 39.2 & 24 & 54.5 & 20 & 39.2 & 11.63 & 0.37 & 15.78 & 0.30 \\
\hline Total & 88 & 100 & 51 & 100 & 44 & 100 & 51 & 100 & 11.70 & 0.35 & 15.80 & 0.32 \\
\hline
\end{tabular}

\subsubsection{Chinese HBSC linked project survey 2008}

The Chinese HBSC linked project survey in 2008 sampled from state schools all over the Beijing metropolitan area. Multi-stage stratified and random cluster sampling method was designed and carried out by the China Institute of Sport Science (CISS) in order to effectively choose the most representative sample.

Selection of the sample districts/counties. The stratified random sample method was used in this stage. The first step of this stage was to stratify all the 16 districts and 2 counties of Beijing area according to geographical location. Two strata, urban area ( 8 districts) and rural area ( 8 districts plus 2 counties) were stratified in this step. The second step was to stratify both the urban and rural areas in terms of social and economic development level. Three levels, good, fair, and poor were used in this step (Beijing Municipal Bureau of Statistics 2007). The last step of this stage was to choose one district (county) randomly from each level divided in the second step in both urban and rural areas. Finally, three districts from urban area and three districts/counties from rural area were selected respectively as the sample district.

Selection of the sample schools. According to the current education policy and regulation in China, children begin the first grade of school at the age of 6 and the timeframe used to determine when a child is eligible to begin school is from September $1^{\text {st }}$ to August $31^{\text {st }}$. For the purposes of this survey therefore, the target population of 11 years old children are mainly in $6^{\text {th }}$ grade (primary school), the 13 years old children are mainly in $8^{\text {th }}$ grade (secondary school), and the 15 years old children are mainly in $10^{\text {th }}$ grade (upper secondary school). The stratified random sample method was used in this stage. All schools in each sample district (county), firstly were divided into three categories on the basis of school conditions (good, fair and poor in terms of school rank and teaching quality) (Beijing Municipal Commission of Education 2007). Then, one primary school, one secondary school and one upper secondary school were selected randomly from each category in each sample district (county). In order to ensure the convenience of the survey, both the secondary school and the upper 
secondary school could be drawn from the same high school which includes both. For those schools which refused or did not answer the invitation to participate in the survey, a substitute school was selected randomly from the same district and of the same school condition. In total, 18 schools were selected for each age group respectively as the sample schools. The response rate of sample schools was $91.5 \%$ and the detailed information of the selection of the sample schools can be seen in Table 2. It should be noted that there were differences in school numbers between China and Finland because the differences of sample class numbers from each school: in China there were three, whilst in Finland there was one, and usually the numbers of students in one class in China is much larger than in Finland.

Selection of the sample classes. The class is the basic sample unit in the HBSC survey. At least three classes were chosen (the average amount of students in one class is about 45 in Beijing) randomly to ensure there were more than 100 students in each sampling age group of each sampling school so that the sample size met the HBSC survey requirement. The cluster sampling method was used for the students in the sampling class. All students in the sample class were invited to attend the school survey.

TABLE 2 Numbers and response rate (\%) of sample schools by age group in Chinese HBSC linked project survey 2008 and Finnish HBSC survey 2006.

\begin{tabular}{|c|c|c|c|c|c|c|c|c|}
\hline & \multicolumn{4}{|c|}{ China 2008} & \multicolumn{4}{|c|}{ Finland 2006} \\
\hline & Total & 11 & 13 & 15 & Total & 11 & 13 & 15 \\
\hline Sample & 54 & 18 & 18 & 18 & 280 & 90 & 90 & 100 \\
\hline Refused or no answer & 5 & 0 & 3 & 2 & 35 & 17 & 13 & 5 \\
\hline Additional schools & 5 & 0 & 3 & 2 & 47 & 23 & 20 & 4 \\
\hline Refused or no answer & 0 & 0 & 0 & 0 & 8 & 5 & 3 & 0 \\
\hline Total & 54 & 18 & 18 & 18 & 284 & 91 & 94 & 99 \\
\hline Response rate (\%) & 91.5 & 100 & 85.7 & 90.0 & 86.9 & 80.5 & 85.5 & 95.2 \\
\hline
\end{tabular}

Finally, a total of 6099 schoolchildren were sampled following the above mentioned three stages. The response rate of sampled students was $98.1 \%$ (Table 3) since 114 students were absent during the survey time for sick leave or other matters (An erratum in article III that the response rate for students was 100\% should be corrected due to absence of information at that time). No student participating in this survey refused to answer the questionnaire. Samples were cleaned in line with the cleaning rule of the HBSC survey protocol if their gender or birth date were missing, or their age was beyond the target range (Currie et al. 2001). The final Chinese data used for analyses in article II contained a total of 5876 schoolchildren aged 11, 13 and 15 years old and in article III and IV contained a total of 2005 schoolchildren aged 15 years old. The characteristics of the valid samples can be seen from Table 4 . 
TABLE 3 Numbers and response rate (\%) of sampled students in Chinese HBSC linked project survey 2008 and Finnish HBSC survey 2006.

\begin{tabular}{|c|c|c|c|c|c|c|c|c|}
\hline & \multicolumn{4}{|c|}{ China 2008} & \multicolumn{4}{|c|}{ Finland 2006} \\
\hline & Total & 11 & 13 & 15 & Total & 11 & 13 & 15 \\
\hline \multicolumn{9}{|l|}{ Total } \\
\hline Sample & 6099 & 2028 & 2022 & 2049 & 6046 & 2064 & 1958 & 2024 \\
\hline Respondents & 5985 & 1987 & 1963 & 2035 & 5334 & 1867 & 1757 & 1710 \\
\hline Response rate $(\%)$ & 98.1 & 98.0 & 97.1 & 99.3 & 88.2 & 90.5 & 89.7 & 84.5 \\
\hline Cleaned data & 5876 & 1941 & 1930 & 2005 & 5255 & 1839 & 1731 & 1685 \\
\hline Valid rate $(\%)$ & 98.2 & 97.7 & 98.3 & 98.5 & 98.5 & 98.5 & 98.5 & 98.5 \\
\hline \multicolumn{9}{|l|}{ Boys } \\
\hline Sample & 2921 & 1018 & 987 & 916 & 2949 & 1004 & 975 & 970 \\
\hline Respondents & 2871 & 999 & 962 & 910 & 2575 & 904 & 862 & 809 \\
\hline Response rate $(\%)$ & 98.3 & 98.1 & 97.4 & 99.3 & 87.3 & 90.0 & 88.4 & 83.4 \\
\hline Cleaned data & 2816 & 975 & 949 & 892 & 2515 & 882 & 843 & 790 \\
\hline Valid rate $(\%)$ & 98.1 & 97.6 & 98.6 & 98.0 & 97.7 & 97.6 & 97.8 & 97.7 \\
\hline \multicolumn{9}{|l|}{ Girls } \\
\hline Sample & 3175 & 1007 & 1035 & 1133 & 3097 & 1060 & 983 & 1054 \\
\hline Respondents & 3111 & 985 & 1001 & 1125 & 2759 & 963 & 895 & 901 \\
\hline Response rate (\%) & 98.0 & 97.8 & 96.7 & 99.3 & 89.1 & 90.8 & 91.4 & 85.5 \\
\hline Cleaned data & 3060 & 966 & 981 & 1113 & 2740 & 957 & 888 & 895 \\
\hline Valid rate $(\%)$ & 98.4 & 98.1 & 98.0 & 98.9 & 99.3 & 99.4 & 99.2 & 99.3 \\
\hline
\end{tabular}

TABLE 4 Valid sample size and mean age (years and decimals) with standard deviation (SD) by gender and age group in Chinese HBSC linked project survey 2008 and Finnish HBSC survey 2006.

\begin{tabular}{|c|c|c|c|c|c|c|c|c|}
\hline \multirow{2}{*}{ Age group } & \multicolumn{4}{|c|}{ China $2008(n=5876)$} & \multicolumn{4}{|c|}{ Finland $2006(n=5255)$} \\
\hline & $\mathrm{n}$ & $\%$ & $\begin{array}{l}\text { Mean } \\
\text { Age }\end{array}$ & SD & $\mathrm{n}$ & $\%$ & $\begin{array}{l}\text { Mean } \\
\text { Age }\end{array}$ & SD \\
\hline \multicolumn{9}{|l|}{11} \\
\hline Boys & 975 & 50.2 & 11.76 & 0.33 & 850 & 47.7 & 11.80 & 0.30 \\
\hline Girls & 966 & 49.8 & 11.72 & 0.32 & 933 & 52.3 & 11.79 & 0.30 \\
\hline Total & 1941 & 100 & 11.74 & 0.32 & 1783 & 100 & 11.80 & 0.30 \\
\hline \multicolumn{9}{|l|}{13} \\
\hline Boys & 949 & 49.2 & 13.75 & 0.33 & 834 & 48.3 & 13.79 & 0.31 \\
\hline Girls & 981 & 50.8 & 13.72 & 0.34 & 891 & 51.7 & 13.77 & 0.32 \\
\hline Total & 1930 & 100 & 13.73 & 0.34 & 1725 & 100 & 13.78 & 0.31 \\
\hline \multicolumn{9}{|l|}{15} \\
\hline Boys & 892 & 44.5 & 15.82 & 0.32 & 790 & 46.9 & 15.79 & 0.33 \\
\hline Girls & 1113 & 55.5 & 15.75 & 0.33 & 895 & 53.1 & 15.76 & 0.31 \\
\hline Total & 2005 & 100 & 15.78 & 0.32 & 1685 & 100 & 15.78 & 0.32 \\
\hline
\end{tabular}




\subsubsection{Finnish HBSC survey 2006}

The data of the Finnish part of the survey used in article III and IV for comparison purposes were obtained from the HBSC survey in Finland in 2006. During the sampling process, the strata were decided based on the province division of Finland. The strata included the northern part of Finland (the provinces of Lappi and Oulu), the central part of Finland (the provinces of Vaasa, KeskiSuomi, Kuopio, Mikkeli, and Pohjois-Karjala), the southern part of Finland (the provinces of Uusimaa, Turku and Pori, Häme and Kymi), and the capital city area (cities of Helsinki, Espoo, Vantaa and Kauniainen). Furthermore, the second level stratum divided those areas by urban and rural communities with the exception of the capital city area due to there being no rural communities. Hence, the final number of the strata used in the sampling procedure was seven (Tynjälä 1999).

In accordance with the target age groups 11,13 , and 15, the nationally representative Finnish samples consisted students in the $5^{\text {th }}, 7^{\text {th }}$ and $9^{\text {th }}$ grades who attend normal education from the state Finnish-speaking compulsory comprehensive schools. A special computer programme was used to choose the sample schools from the Finnish school register using cluster sampling with Probability Proportional to Size (PPS) of schools method (the size of schools was taken into account). Additional samples were recruited to ensure the optimal sample size as per the requirement. In case some selected schools refused to take part in the survey or did not return the questionnaire in the requested time period. The substitute school was chosen as the first school from the extra list within the same province and of the same community category as the none-responding school (Tynjälä 1999). The response rate of sampled schools was $86.9 \%$ and the detailed information on Finnish sampled schools is presented in Table 2.

One class was randomly selected within each sample school, except for only a few cases where more than one class were chosen from the same school in the sample (Tynjälä 1999). Finally, a total of 6046 students were selected to take part in the survey. The response rate of sampled students was $88.2 \%$ (Table 3). Samples were cleaned in line with the cleaning rule of the HBSC survey protocol if their gender or birth date were missing, or their age was beyond the target range (Currie et al. 2001). The final Finnish samples included a total of 5255 pupils aged 11, 13 and 15 years old, among which 1685 15-year-old pupils (boys $46.9 \%$ ) were used for analyses in article III and IV. The characteristics of the valid samples can be seen from Table 4 .

\subsection{Data collection}

Test-retest reliability study in Beijing, China 2008. The first part of test-retest study was administered by one researcher from the CISS and one class teacher from the school during an ordinary class hour. The students were instructed how to fill in the questionnaire by the researcher and they were not informed about the 
forthcoming retest. Three weeks later the second part of test-retest study was conducted through an identical procedure. All students participating in the test and retest were asked to write their student identity number on the questionnaire to permit matching the test and retest questionnaires. Student's participation in the test and retest was totally voluntary and the questionnaire, as well as the student identity number, can only be accessed by the researcher. Students were also informed that only the researcher will read their answers. Verbal consent was sought from all the participants, the head teachers of the classes, and the principle of the school. The test and retest were done at the end of October and at the middle of November in 2008.

Chinese HBSC linked project survey 2008. The Chinese HBSC linked project was carried out by CISS with the collaboration with the Research Centre for Health Promotion at the University of Jyväskylä and Beijing Academy of Educational Science in December 2008. All students in the selected sample classes were asked to complete a self-report questionnaire during a normal school class (45 minutes) with a teacher and/or researcher administering. The students were first instructed how to fill in the questionnaire. Student's participation in the survey was voluntary and anonymous, and the questionnaire could only be accessed by researcher. Students were also informed that only the researcher will read their answers. After completion of the survey questionnaire, they were collected and sent to CISS with additional information on the name of sample school and the amount of sample classes and students. Both the test-retest reliability study and the Chinese HBSC linked project survey were approved by the ethics committee of CISS and the Research Centre for Health Promotion at the University of Jyväskylä. All data from the above mentioned two studies were entered into Epidata 3.1 with double entry by an independent professional data service company and validated by the researcher from CISS.

Finnish HBSC survey 2006. The Finnish HBSC study was conducted by the Research Centre for Health Promotion at the Department of Health Sciences of the University of Jyväskylä. Despite the similarities in the data collection procedure of the Chinese survey mentioned above, there were a few discrepancies in the Finnish survey. Firstly, before the survey in the classroom, the survey questionnaires were mailed to each sample school with instructions on how to implement the data collection in classrooms. Secondly, students were asked to answer the questionnaire with the administration of the teacher and put their questionnaire into an envelope, seal it and hand it in to the teacher. After the class period, the teacher put those envelopes into a bigger envelope (with the address and prepaid postage cost) and sent the package to the Research Centre for Health Promotion at the University of Jyvaskyla. Finally, those packages were opened by the researchers in the University of Jyväskylä and registration information were added, for instance, the arrival data of the package, number of respondents and students absent from the class. Furthermore, students' questionnaires were numbered for later checking. Following that procedure, the questionnaires were sent to the Computer Centre of the University of Jyväskylä for coding through the photoelectric read method and preliminary data file 
formation. The Finnish HBSC study was approved by the Finnish National Board of Education (FNBE) and the Trade Union of Education in Finland.

\subsection{Instrument and variables}

In terms of the research protocol, the international standard questionnaire contains mandatory questions which are used in all HBSC member countries and several packages of optional questions on specific topics which can be added in to the questionnaire by each participating country according to their purpose. Furthermore, country-specific items regarding the national importance or interests may also be included in the questionnaire.

The HBSC survey questionnaire covers a range of indicators measuring health behaviours, such as eating and dieting, physical activity, sedentary behaviour, oral health and health outcomes, such as self-rated health, injuries, life satisfaction etc., risk behaviour, such as smoking, alcohol use, bullying, sexually behaviour etc., as well as demographic factors, social background (e.g., family structure and socioeconomic status), and social context (e.g., family and peer culture, and school environment) (Roberts et al. 2009).

The questionnaires used in the Chinese surveys were based on the English version of the questionnaire used in the Finnish HBSC Survey in 2006. The questionnaire was firstly translated from English to Chinese by two researchers independently and re-translated from Chinese to English by other professional translators to check for any discrepancies. All mandatory questions based on the international HBSC research protocol (Currie et al. 2001) were included and kept identical as they were in the Finnish survey questionnaires. Meanwhile, a few additional items with country-specific topics were also added. The detailed information of survey items used in the present study can be found from appendix 1.

\subsubsection{Selected items in test-retest reliability study}

The questionnaire used in the test-retest study in Beijing 2008 contained 102 questions (items) and the same questionnaire was used in both the test and the retest. Of those items, a total of 23 items concerning physical activity (4 items), sedentary behaviour (8 items), sleep ( 4 items) and substance use (7 items) were examined in the test-retest reliability study. The detailed information of items and their response alternatives can be found in Table 5 . 
TABLE 5 The selected items related to health behaviours and their response alternatives in test-retest reliability study in Beijing, China 2008.

\begin{tabular}{|c|c|}
\hline Items & Response Alternatives \\
\hline \multicolumn{2}{|l|}{ Physical Activity } \\
\hline $\begin{array}{l}\text { 1. Over the past } 7 \text { days, on how many days were you physically } \\
\text { active for a total of at least } 60 \text { minutes per day? }\end{array}$ & 0 day; $1 ; 2 ; 3 ; 4 ; 5 ; 6 ; 7$ days. \\
\hline $\begin{array}{l}\text { 2. Over a typical or usual week, on how many days are you physi- } \\
\text { cally active for a total of at least } 60 \text { minutes per day? }\end{array}$ & 0 day; $1 ; 2 ; 3 ; 4 ; 5 ; 6 ; 7$ days. \\
\hline $\begin{array}{l}\text { 3. OUTSIDE SCHOOL CLASS: How OFTEN do you usually take } \\
\text { physical exercise in your free time so that you lose your breath or } \\
\text { sweat? }\end{array}$ & $\begin{array}{l}\text { Daily; 4-6 times a week; } 2-3 \text { times a } \\
\text { week; Once a week; Once a month; Less } \\
\text { than once a month; Never. }\end{array}$ \\
\hline $\begin{array}{l}\text { 4. OUTSIDE SCHOOL CLASS: How many HOURS a week do you } \\
\text { usually take physical exercise in your free time so that you lose } \\
\text { your breath or sweat? }\end{array}$ & $\begin{array}{l}\text { None; Approx. half an hour; Approx. an } \\
\text { hour; Approx. 2-3 hours; Approx. 4-6 } \\
\text { hours; Seven hours or more. }\end{array}$ \\
\hline \multicolumn{2}{|l|}{ Sedentary Behaviour } \\
\hline $\begin{array}{l}\text { How many hours a day do you usually do the following things in your } \\
\text { free time? }\end{array}$ & None; \\
\hline 5. Watch TV or videos or DVDs on school days & Approx. $h$ \\
\hline 6. Watch TV or videos or DVDs at weekends & Approx. an hour; \\
\hline $\begin{array}{l}\text { 7. Use a computer for playing games or use console games on } \\
\text { school days }\end{array}$ & Approx. 2 hours; \\
\hline $\begin{array}{l}\text { 8. Use a computer for playing games or use console games at } \\
\text { weekends }\end{array}$ & Approx. 3 hours; \\
\hline $\begin{array}{l}\text { 9. Use a computer for: chatting online, internet, emailing, home- } \\
\text { work etc. on school days }\end{array}$ & Approx. 4 hours; \\
\hline $\begin{array}{l}\text { 10. Use a computer for: chatting online, internet, emailing, home- } \\
\text { work etc. at weekends }\end{array}$ & Approx. 5 hours; \\
\hline $\begin{array}{l}\text { 11. Spend doing your school homework out of school hours on } \\
\text { school days }\end{array}$ & Approx. 6 hours; \\
\hline $\begin{array}{l}\text { 12. Spend doing your school homework out of school hours at } \\
\text { weekends } \\
\text { Sleep }\end{array}$ & Approx. 7 hours or more. \\
\hline $\begin{array}{l}\text { 13. When do you usually go to bed if you have to go to school in } \\
\text { the next morning? }\end{array}$ & $\begin{array}{l}\text { No later than } 21: 00 ; 21: 30 ; 22: 00 ; 22: 30 ; \\
\text { 23:00; } 23: 30 ; 24: 00 ; 00: 30 ; 01: 00 ; 01: 30 ; \\
02: 00 \text { or later. }\end{array}$ \\
\hline $\begin{array}{l}\text { 14. When do you usually go to bed at weekends or during holi- } \\
\text { days? }\end{array}$ & $\begin{array}{l}\text { No later than } 21: 00 ; 21: 30 ; 22: 00 ; 22: 30 ; \\
\text { 23:00; } 23: 30 ; 24: 00 ; 00: 30 ; 01: 00 ; 01: 30 ; \\
02: 00 ; 02: 30 ; 03: 00 ; 03: 30 ; 04: 00 \text { or later. }\end{array}$ \\
\hline 15. When do you usually wake up on school mornings? & $\begin{array}{l}\text { No later than 05:00; 05:30; 06:00; 06:30; } \\
\text { 07:00; 07:30; 08:00 or later. }\end{array}$ \\
\hline 16. When do you usually wake up at weekends? & $\begin{array}{l}\text { No later than 07:00; 07:30; 08:00; 08:30; } \\
\text { 09:00; 09:30; 10:00; 10:30; 11:00; 11:30; } \\
\text { 12:00; 12:30; 13:00; 13:30; 14:00 or later }\end{array}$ \\
\hline \multicolumn{2}{|l|}{ Risk Behaviour: substance use } \\
\hline 17. Have you ever smoked? & Yes; No. \\
\hline 18. How often do you smoke at present? & $\begin{array}{l}\text { Every day; Every week, but not daily; } \\
\text { Less than once a week; I do not smoke. }\end{array}$ \\
\hline $\begin{array}{l}\text { 19. How many cigarettes, pipefuls or cigars have you smoked until } \\
\text { now? } \\
\text { At present, how often do you drink..? }\end{array}$ & None; One; Approx. 2-50; More than 50. \\
\hline 20. Beer & Daily; At least once a week; \\
\hline 21. Wine & At least once a month; Rarely; \\
\hline 22. Strong liquors & Never. \\
\hline $\begin{array}{l}\text { 23. Have you ever had so much alcohol that you have been really } \\
\text { drunk? }\end{array}$ & $\begin{array}{l}\text { Never; Yes, once; Yes, } 2-3 \text { times; Yes, } 4- \\
10 \text { times; Yes, more than } 10 \text { times. }\end{array}$ \\
\hline
\end{tabular}




\subsubsection{Measurements of alcohol use}

Alcohol consumption

Alcohol consumption was assessed by the item 'At present, how often do you have the following alcoholic drinks? Also include those times when you only drink a very small amount'. For each type of beverage: beer, wine, strong liquor, mild alcohol drinks, and any other drink that contains alcohol, the possible answers were: 'daily', 'at least once a week', 'at least once a month', 'less', and 'never'. Monthly alcohol use was defined as drinking any alcohol at least once a month.

\section{Onset of alcohol use}

The initiation of alcohol use was examined by asking the questions 'At what age did you drink alcohol (more than a small amount) for the first time?' The answers were: 'never', '11 years old or less', '12 years old', '13 years old', '14 years old', '15 years old or older'. Early onset of alcohol use was defined as first drinking alcohol at age 13 or younger.

\section{Drunkenness}

Frequency of drunkenness was assessed by asking the respondents whether they had ever had so much alcohol that they had been really drunk. The response alternatives were: 'never', 'yes, once', 'yes, 2-3 times', 'yes, 4-10 times' and 'yes, more than 10 times'. Drunkenness was defined as 2-3 or more times.

\section{Onset of drunkenness}

The initiation of drunkenness was examined by asking the questions 'At what age you were drunk for the first time?' The answers were: 'never', '11 years old or less', '12 years old', '13 years old', '14 years old', '15 years old or older'. Early onset of drunkenness was defined as those respondents who reported they were first drunk at age 13 or younger.

\subsubsection{Measurements of smoking}

\section{Ever smoked}

The issue of whether students had ever smoked was examined by asking the question 'have you ever smoked (at least one cigarette, cigar or a pipeful)?' The answers were: 'yes', or 'no'. Respondents who answered yes were defined as ever smoked.

\section{Present status of smoking}

The present status of smoking was assessed by asking the students 'how often do you smoke at present?' The response alternatives were: 'every day', 'every week, but not daily', 'less than once a week', 'I do not smoke'. A daily smoker was defined as those students reporting that they smoke every day. All respondents of this item were defined as a current smoker except for those who answered that they do not smoke, or respondents with missing answers. 
Onset of smoking

The initiation of smoking was examined by the item asking 'at what age did you smoke a cigarette (more than a puff) for the first time?' The answers were: 'never', '11 years old or less', '12 years old', '13 years old', '14 years old', '15 years old or older'. Early onset of smoking was defined as those respondents who reported first smoking at age 13 years or younger.

\subsubsection{Measurements of SES in both countries}

\section{Family Affluence Scale (FAS)}

The 'Family Affluence Scale' (FAS) has been used to examine and explain socioeconomic inequalities in the HBSC study for more than 10 years and the FAS II has been shown as a reliable and valid SES measure for adolescents (Currie et al. 2008b). Although the reliability and validity of the FAS II have not been examined in Finland, validation studies have been done extensively in many European countries in which the situation is similar to Finland (Andersen et al. 2008; Boyce et al. 2006; Molcho et al. 2007; Schnohr et al. 2008; Wardel et al. 2002). The FAS II was used as a measure of SES in this study. The items, response categories, codes and analyses strategy of FAS II used in the present study are as follows:

"Does your family own a car, van or truck?"

Response categories were: No $(=0)$; Yes, one $(=1)$; Yes, two or more $(=2)$.

"Do you have your own bedroom for yourself?"

Response categories were: No $(=0)$; Yes $(=1)$.

"During the past 12 months, how many times did you travel away on holiday with your family?"

Response categories were: Not at all $(=0)$; Once $(=1)$; Twice $(=2)$; More than twice $(=3)$.

"How many computers does your family own?"

Response categories were: None $(=0)$; One $(=1)$; Two $(=2)$; More than two $(=3)$.

According to Currie and her colleagues (2008b), a composite FAS score was calculated for each respondent based on his or her answers to these four items. Following previous HBSC surveys, the two highest response categories (' 2 ' and ' 3 or more') of the last two items (holidays and computers) were combined. Three groups were categorized in terms of the composite FAS score, in which FAS low (score $=0-3$ ) indicated low affluence, FAS medium (score $=4,5)$ indicated middle affluence, and FAS high (score $=6,7)$ indicated high affluence.

Perceived family wealth (PFW)

Perceived socioeconomic status of the household was enquired by asking the students' own perception of their family financial situation. The responses were: 'very well off financially', 'quite well off financially', 'average', 'not very well off financially', and 'not at all well off financially'. The indicator of perceived family wealth was divided into three categories, perceived low family wealth 
(not very well off, not well off at all), perceived medium family wealth (average), and perceived high family wealth (very well off, quite well off).

\section{Parental employment status (PES)}

There were two questions assessing parental employment status (PES). First, students were asked to describe whether their father and mother go to work. If not, the reason why the respondent's father and/or mother do not go to work was asked. During the analyses, the two questions were combined for grouping. The students were grouped in the high employment category (both father and mother work, and father or mother works and the other one takes care of family member), the low employment category (neither parent works), and the others in the middle employment category.

After calculating the distribution of different measures of SES by country, we found that there are very few cases of low PES group in both China $(1.9 \%, \mathrm{n}=$ 38 ) and Finland $(4.2 \%, \mathrm{n}=70)$, and low PFW group in Finland $(4.3 \%, \mathrm{n}=72)$. Thus we decided to use FAS as the SES measures for analyses in this study (III and IV).

\subsubsection{Chinese specific SES indicators}

Apart from the above mentioned FAS II, PFW and POS used in article II, III, and IV, several other indicators were employed for analyses in article II for the purpose of validation of the FAS II in China, which were not used in the Finnish surveys but in the Chinese surveys. These additional indicators were:

Urban and rural

According to the sampling procedure, the information of selected schools in the survey can be retrieved, and the respondents were divided into urban and rural according to the school position.

Parents' educational level

Students were asked to choose their father's and mother's highest level of education from the 7 alternatives: under primary school, primary school, secondary school, upper secondary school, junior college, university (bachelor level), and graduate or more. During the analyses, the parents' education were divided into three categories, low education level (under the primary school, primary school, secondary school), medium education level (upper second school, junior college), and high education level (university, graduate or more), for father and mother respectively.

School location

The respondents were categorized into three SES groups: good, fair and poor, according to their school's location in districts with different socioeconomic development (Beijing Municipal Bureau of Statistics 2007). 


\subsection{Statistical methods}

Except for articles I and II, in which only Chinese data were used, all analyses were performed separately for China and Finland and most of these analyses were also separated for boys and girls. Descriptive statistics were used to show the characteristics of the study samples and the distribution of the variables. The association between nominally scaled variables was analysed using crosstabulations, and its statistical significance was tested by the Pearson's Chisquare test. Further details regarding statistical methods are provided below.

For article I, overall stability rate of items were given by the proportion of subjects showing no response shift on the item between test and retest. The frequency of response shifts of 1, 2 and 3 or more categories were also computed. The test-retest reliability of all selected items was estimated using the single measure of Intraclass Correlation Coefficients (ICC), which were computed as devised by Shrout \& Fleiss (1979), through case 2 (using a two-way random model with an absolute agreement type), with $95 \%$ confidence interval (CI), for all respondents, and stratified by gender and age. These values were considered significantly different if their $95 \%$ confidence intervals (CIs) did not overlap. According to Landis \& Koch (1977), the strength of test-retest agreement for ICC is classified as follows: below 0.20 is poor; 0.21 to 0.40 shows a fair agreement; 0.41 to 0.60 indicates a moderate degree of agreement; 0.61 to 0.80 means substantial agreement; and 0.81 to 1 indicates almost perfect agreement. These classifications were used to interpret the results. The items about substance use were evaluated only for the adolescents aged 15 years old due to the absence in this behaviour among 11 year-old respondents.

For article II, item completion rate was computed for FAS II items and total FAS scores as well as the other two SES indicators (parental educational level and perceived family wealth). Pearson's Chi-square test was used to compare the completion rate among those SES measures. The internal consistency of the FAS II items and item-rest coefficient were examined with the Cronbach's alpha coefficient. Test-retest reliability was evaluated by ICC as mentioned before. Spearman correlations were used to assess the association between individual FAS II item, the total FAS scores and FAS category. In order to examine whether the four FAS II items are to reflect a general underlying dimension of affluence, the fit of a one-factor solution was tested with confirmatory factor analysis (CFA) for categorical data using the Mean- and Variance-adjusted Weighted Least Square (WLSMV) estimator. The primary fit indices included the Comparative Fit Index (CFI) and the weighted root mean square residual (WRMR), as recommended in earlier publications (Finney \& DiStefano 2006). A CFI $\geq 0.95$ and WRMR $<1$ indicates a good fit to the data (Yu \& Muthén 2002). The external validity of FAS II was evaluated by using Spearman rank correlations to assess the association between the total FAS scores with parental educational level and perceived family wealth. In addition, two ordinal logistic regression models were used for analysing the association between FAS category and oth- 
er SES indicators. The unadjusted odds ratio (OR) from model 1 (univariate ordinal regression model) and the adjusted OR from model 2 (included all independent variables) were computed respectively. Furthermore, construct validity of FAS II was investigated with the Graphical Log Linear Rasch Model (GLLRM) with Differential Item Function (DIF) and Local Dependence (LD).

In articles III and IV, subjects with missing values on alcohol use and smoking were treated as none presence of drinking or smoking to avoid overestimating the prevalence of adolescents' substance use. Binary logistic regression models were used to study the association between family affluence scale and alcohol use (III) (including monthly alcohol use, early onset of alcohol use, drunkenness, and early onset of drunkenness) and smoking (IV) (including ever smoked, daily smoker, current smoker and early onset of smoking) of adolescents. Odds ratios (OR) with their corresponding 95\% confidence intervals (CI) were calculated for the regression model. In each model, the associations were evaluated by gender and country, and either the low or the high FAS group served as the reference category with odds ratios and 95\% CIs being computed for the other two FAS groups for comparison.

For all the above mentioned analyses, a $p$ value of $<0.05$ or $95 \%$ CIs do not overlap was considered statistically significant. The CFA was analysed by MPlus 6.1 (Muthén \& Muthén 2010). The GLLRM was performed by DIGRAM 2.0 (Kreiner 2009). All the other analyses were done by using Predictive Analytics Software (PASW, formerly SPSS), version 18.0 (I, II and III) or version 20.0 (IV) (SPSS, Inc., Chicago, IL, US). 


\section{RESULTS}

\subsection{The test-retest reliability of selected survey items (Study I)}

The first article was based on the pilot study for the HBSC linked project in China of which the objectives were to examine the feasibility of the HBSC survey and the test-retest reliability of selected items from the HBSC survey questionnaire in a sample of adolescents in Beijing, China.

Overall, none of students refused to fill in the questionnaire and all respondents could complete the whole questionnaire within one school hour (45 minutes) in both test and retest. During the survey process, participating students did not ask any questions or interpretation concerning the items used in the questionnaire during the data collection. Furthermore, the school administrators and teachers gave their consent to the content of questionnaire and the procedure of the survey after reading the survey protocol and the questionnaire.

The proportions of no response shift between test and retest varied from $32 \%$ for the item measuring computer use at weekends, to $92 \%$ for the three items on smoking behaviour. At least $68 \%$ of the respondents gave an answer in the same or an adjacent category for all selected indicators (Figure 1).

The values of ICC for all respondents were stratified by gender and age (Tables 6 and 7). Overall, the values of ICC of the selected items ranged from 0.33 to 0.85 , with the lowest value for the item regarding using a computer on school days, and the highest value for items on how many cigarettes ever smoked and pertaining to the question 'have you ever been drunk?' Of all the 23 items evaluated in this study, according to Landis and Koch divisions of agreement (1977), 6 items (26\%) showed a moderate reliability, 12 items (52\%) displayed a substantial reliability and 4 items $(17 \%)$ indicated almost perfect reliability. By gender, the values of ICC varied from 0.19 to 0.96 for girls and 0.42 to 0.85 for boys. The items of the highest and lowest ICC for girls are not consistent with the items for boys. By age groups, ICC ranged from 0.38 to 0.86 for 11 year-old respondents and 0.16 to 0.85 for 15 year-old respondents. 
Item (numbers of response alternatives) $\square$ no shift 曾 one category $\mathbf{Q}$ two categories $\square$ three categories or more

*How many cigarettes ever smoked (4)

*How oftern smoke at present (4)

*Have ever smoked (2)

*How often drink strong liquors (5)

*Have ever been drunk (5)

When wake up on school mornings (7)

*How often drink beer $(5)$

*How often drink wine (5)

Playing PC or console games on school days (9)

Watching TV on school days (9)

Doing homework on school days (9)

MVPA $^{\mathrm{a}}$ in the last 7 days (8)

When go to bed on school days (11)

MVPA in the usual week (8)

Playing PC or console games at weekends (9)

Doing homework at weekends (9)

When go to bed at weekends (15)

Using PC on school days (9)

VPA ${ }^{\mathrm{b}}$ per week (6)

When wake up at weekends (15)

$\mathrm{VPA}^{\mathrm{b}}$ frequency (7)

Watching TV at weekends (9)

Using PC at weekends (9)
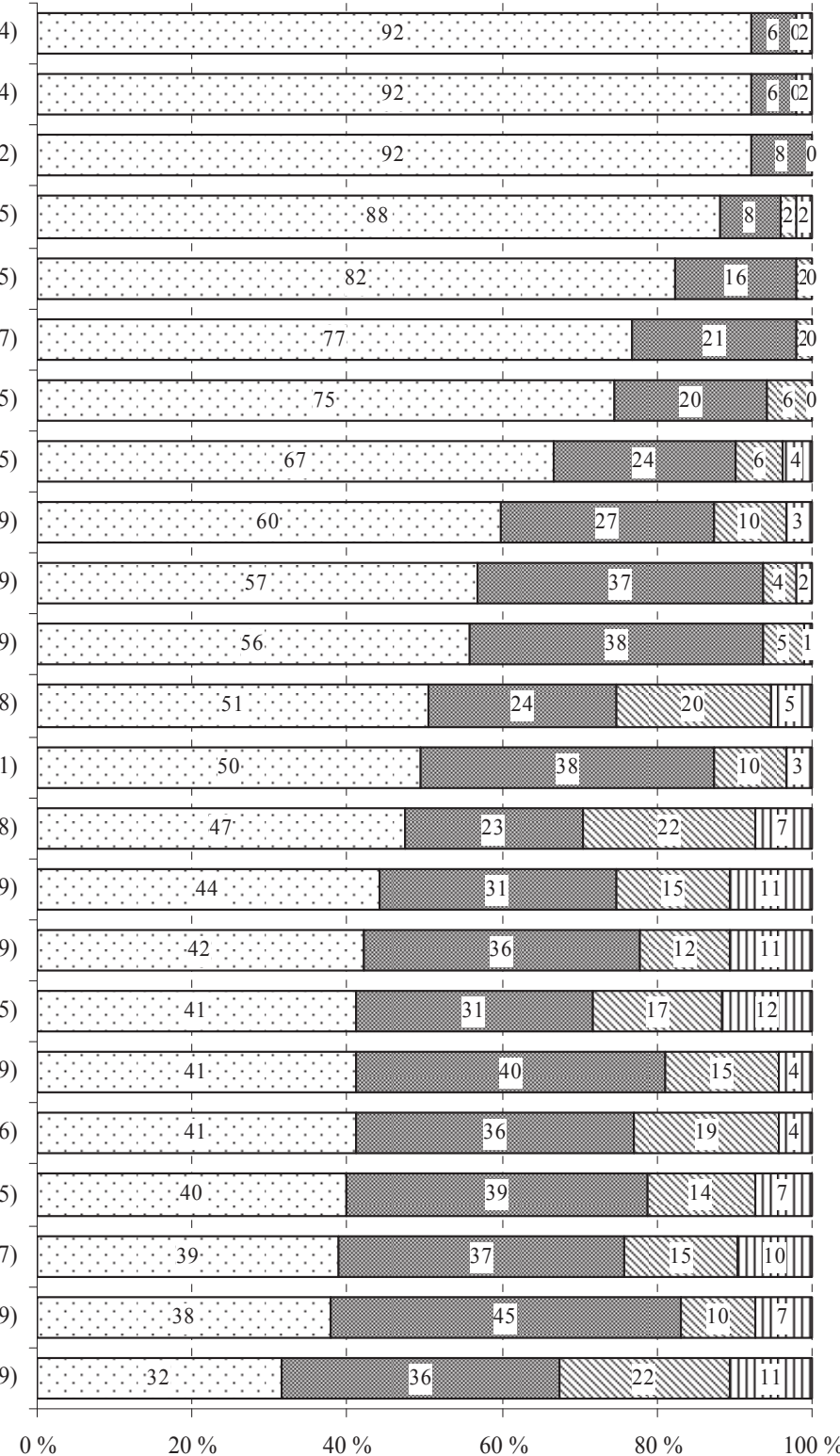

*Items were only computed for respondents aged 15 years old $(\mathrm{n}=51)$ aModerate to Vigorous Physical Activity, bVigorous Physical Activity

FIGURE 1 Frequencies of test-retest shifts on all selected items in test-retest reliability study in Beijing, China 2008, sorted according to the frequencies of no response shift, descending order $(n=95)$. 


\begin{tabular}{|c|c|c|c|c|c|c|c|c|c|c|}
\hline \multirow[b]{2}{*}{ Items } & \multicolumn{2}{|c|}{ All $(n=95)$} & \multicolumn{2}{|c|}{ Girls $(n=44)$} & \multicolumn{2}{|c|}{ Boys $(n=51)$} & \multicolumn{2}{|c|}{11 years $(n=44)$} & \multicolumn{2}{|c|}{15 years $(n=51)$} \\
\hline & ICC & $95 \% \mathrm{CI}$ & ICC & $95 \% \mathrm{CI}$ & ICC & $95 \% \mathrm{CI}$ & ICC & $95 \% \mathrm{CI}$ & ICC & $95 \% \mathrm{CI}$ \\
\hline \multicolumn{11}{|l|}{ Physical Activity } \\
\hline MVPAa last 7 days $^{2}$ & 0.82 & $0.74-0.88$ & 0.87 & $0.77-0.93$ & 0.79 & $0.66-0.88$ & 0.81 & $0.67-0.89$ & 0.79 & $0.66-0.88$ \\
\hline MVPAa usual week & 0.74 & $0.64-0.82$ & 0.76 & $0.60-0.86$ & 0.73 & $0.57-0.83$ & 0.77 & $0.61-0.87$ & 0.71 & $0.54-0.82$ \\
\hline $\mathrm{VPA}^{\mathrm{b}}$ frequency & 0.68 & $0.55-0.77$ & 0.73 & $0.56-0.85$ & 0.61 & $0.41-0.76$ & 0.72 & $0.54-0.84$ & 0.62 & $0.42-0.76$ \\
\hline VPA $^{b}$ time per week & 0.57 & $0.42-0.66$ & 0.66 & $0.45-0.80$ & 0.50 & $0.27-0.68$ & 0.58 & $0.35-0.75$ & 0.56 & $0.34-0.72$ \\
\hline \multicolumn{11}{|l|}{ Sedentary Behaviour } \\
\hline Watching TV on school days & 0.72 & $0.61-0.81$ & 0.91 & 0.83-0.95 & 0.51 & $0.28-0.69$ & 0.86 & $0.76-0.92$ & 0.57 & $0.36-0.73$ \\
\hline Watching TV at weekends & 0.74 & $0.63-0.83$ & 0.77 & $0.62-0.87$ & 0.70 & $0.53-0.82$ & 0.80 & $0.66-0.89$ & 0.68 & $0.49-0.80$ \\
\hline $\begin{array}{l}\text { Playing PC or console games on school } \\
\text { days }\end{array}$ & 0.54 & $0.38-0.67$ & 0.56 & $0.32-0.73$ & 0.53 & $0.30-0.70$ & 0.56 & $0.32-0.73$ & 0.52 & $0.29-0.70$ \\
\hline $\begin{array}{l}\text { Playing PC or console games at week- } \\
\text { ends }\end{array}$ & 0.69 & $0.57-0.78$ & 0.47 & $0.20-0.67$ & 0.83 & $0.72-0.90$ & 0.79 & $0.64-0.88$ & 0.57 & $0.35-0.73$ \\
\hline Using PC on school days & 0.33 & $0.14-0.50$ & 0.19 & $-0.11-0.46$ & 0.45 & $0.20-0.64$ & 0.38 & $0.10-0.60$ & 0.28 & $0.01-0.51$ \\
\hline Using PC at weekends & 0.50 & $0.33-0.64$ & 0.37 & $0.09-0.60$ & 0.58 & $0.36-0.73$ & 0.83 & $0.71-0.90$ & 0.16 & $-0.12-0.41$ \\
\hline Doing homework on school days & 0.78 & $0.68-0.85$ & 0.81 & $0.68-0.89$ & 0.74 & $0.59-0.85$ & 0.75 & $0.59-0.86$ & 0.73 & $0.57-0.84$ \\
\hline Doing homework at weekends & 0.73 & $0.62-0.82$ & 0.69 & $0.49-0.82$ & 0.76 & $0.62-0.86$ & 0.54 & $0.29-0.72$ & 0.79 & $0.65-0.87$ \\
\hline \multicolumn{11}{|l|}{ Sleep } \\
\hline When go to bed on school days & 0.75 & $0.65-0.83$ & 0.76 & $0.60-0.86$ & 0.73 & $0.57-0.84$ & 0.68 & $0.48-0.81$ & 0.60 & $0.39-0.75$ \\
\hline When go to bed at weekends & 0.64 & $0.50-0.67$ & 0.64 & $0.43-0.79$ & 0.62 & $0.41-0.76$ & 0.51 & $0.26-0.70$ & 0.58 & $0.36-0.74$ \\
\hline When wake up on school mornings & 0.77 & $0.68-0.84$ & 0.79 & $0.65-0.88$ & 0.76 & $0.62-0.86$ & 0.81 & $0.68-0.89$ & 0.73 & $0.57-0.84$ \\
\hline When wake up at weekends & 0.83 & $0.75-0.88$ & 0.82 & $0.69-0.90$ & 0.84 & $0.73-0.90$ & 0.85 & $0.74-0.92$ & 0.78 & $0.65-0.87$ \\
\hline
\end{tabular}

aModerate to Vigorous Physical Activity, bVigorous Physical Activity, significant differences by gender or age group are in bold text $(p<0.05)$. 
TABLE 7 ICC values of selected HBSC survey items about alcohol use and smoking of adolescents aged 15 years by gender in test-retest reliability study in Beijing, China 2008.

\begin{tabular}{|c|c|c|c|c|c|c|}
\hline & \multicolumn{2}{|c|}{ All $(n=51)$} & \multicolumn{2}{|c|}{ Girls $(n=20)$} & \multicolumn{2}{|c|}{ Boys $(n=31)$} \\
\hline & ICC & $95 \% \mathrm{CI}$ & ICC & $95 \% \mathrm{CI}$ & ICC & $95 \% \mathrm{CI}$ \\
\hline Have ever smoked & 0.75 & $0.60-0.85$ & $\mathrm{~N} / \mathrm{A}$ & & 0.63 & $0.36-0.80$ \\
\hline How often smoke at present & 0.50 & $0.27-0.68$ & $\mathrm{~N} / \mathrm{A}$ & & 0.48 & $0.15-0.71$ \\
\hline How many cigarettes ever smoked & 0.85 & $0.75-0.91$ & $\mathrm{~N} / \mathrm{A}$ & & 0.81 & $0.64-0.90$ \\
\hline How often drink beer & 0.80 & $0.67-0.88$ & 0.96 & $0.90-0.98$ & 0.82 & $0.66-0.91$ \\
\hline How often drink wine & 0.53 & $0.30-0.70$ & 0.70 & $0.39-0.87$ & 0.42 & $0.09-0.67$ \\
\hline How often drink strong liquors & 0.44 & $0.19-0.64$ & 0.76 & $0.49-0.90$ & 0.64 & $0.37-0.81$ \\
\hline Have ever been drunk & 0.85 & $0.76-0.91$ & 0.83 & $0.61-0.93$ & 0.85 & $0.72-0.93$ \\
\hline
\end{tabular}

$\mathrm{N} / \mathrm{A}=$ Not applicable due to lack of variance.

The reliability of the four items assessing Moderate to Vigorous Physical Activity (MVPA) and Vigorous Physical Activity (VPA) ranged from moderate (ICC = 0.57 ) to almost perfect agreement (ICC $=0.82$ ). The lowest reliability was found in the item measuring VPA time per week, and the highest reliability in the item relating to MVPA in the last 7 days. No statistically significant differences were found either by gender or by age group, although the ICC value may differ.

Of the eight items examining the sedentary behaviours, seven of them showed a moderate to a substantial agreement. The question inquiring about using a computer on school days was the only item which indicated a fair agreement, and expressed the lowest value of ICC (0.33) for all respondents among all the selected items in this study. Significant gender differences were found in items on watching TV on school days and playing computer or console games at weekends $(p<0.05)$. Meanwhile, significant age differences were found in items on watching TV on school days and using a computer at weekends $(p<0.05)$.

All items on sleep patterns demonstrated at least substantial reliability, especially for the item on when children wake up at weekends, for which the reliability is almost perfect (ICC $=0.83$ ). On the contrary, the lowest value of ICC was found for the item on when children go to bed at weekends (ICC = $0.64)$. There were no gender and age differences in these items.

The items on substance use were evaluated only for students aged 15 years old. Four items indicated a substantial to almost perfect reliability and the values of ICC varied from 0.75 to 0.85 . The other three items showed at least moderate reliability and the lowest reliability was exhibited by the question of how often do you drink strong liquors $($ ICC $=0.44)$. None of the girls in this study reported they have ever smoked, so this constant result lead to the value of ICC for three items on smoking to being not applicable due to lack of variance. 


\subsection{The reliability and validity of the Family Affluence Scale (Study II)}

The objectives of the second article were to assess the completion rate, reliability and validity of the HBSC FAS II items, serving as the vital indicators of SES measures in later analyses concerning socioeconomic inequality in smoking and alcohol use among Chinese adolescents. In accordance with these objectives, the results were derived from two studies, including the test-retest reliability of the FAS II items from the pilot test-retest study with 95 students conducted in Beijing in October 2008, and the HBSC linked project survey with 5876 school-aged children carried out in Beijing between November and December 2008.

\subsubsection{Test-retest reliability}

Table 8 reveals the values of ICC of the FAS II items, as well as the FAS score and category, for all respondents and by gender and age. Overall, the ICC values of the FAS II items, FAS score and FAS category ranged from 0.76 to 0.95 , with the lowest value for the item regarding family holidays, and the highest value for the item asking about own bedroom. According to Landis and Koch divisions of agreement (1977), all four items of FAS II as well as the FAS score and category showed at least substantial test-retest reliability and there were no gender and age differences of the agreement.

TABLE 8 ICC value of FAS II items, FAS score and FAS category by gender and age in test-retest reliability study in Beijing, China 2008.

\begin{tabular}{llllllllllc}
\hline & \multicolumn{2}{l}{ All $(\mathrm{N}=95)$} & \multicolumn{3}{l}{ Boys $(\mathrm{n}=51)$} & \multicolumn{2}{l}{ Girls $(\mathrm{n}=44)$} & \multicolumn{2}{l}{$11(\mathrm{n}=44)$} & \multicolumn{2}{l}{$15(\mathrm{n}=51)$} \\
\cline { 2 - 13 } & ICC & $95 \%$ CI & ICC & $95 \%$ CI & ICC & $95 \%$ CI & ICC & $95 \%$ CI & ICC & $95 \%$ CI \\
\hline Family car & 0.88 & $0.83-0.92$ & 0.85 & $0.75-0.91$ & 0.92 & $0.86-0.96$ & 0.81 & $0.68-0.89$ & 0.92 & $0.86-0.95$ \\
Own bedroom & 0.95 & $0.93-0.97$ & N/A & & 0.92 & $0.86-0.96$ & 0.79 & $0.65-0.88$ & N/A & $/$ \\
Family holidays & 0.76 & $0.66-0.83$ & 0.72 & $0.55-0.83$ & 0.77 & $0.61-0.87$ & 0.78 & $0.64-0.88$ & 0.64 & $0.44-0.79$ \\
No. of computers & 0.77 & $0.67-0.84$ & 0.77 & $0.63-0.86$ & 0.76 & $0.61-0.86$ & 0.73 & $0.56-0.84$ & 0.79 & $0.66-0.87$ \\
FAS score & 0.88 & $0.83-0.92$ & 0.85 & $0.75-0.91$ & 0.92 & $0.84-0.96$ & 0.83 & $0.70-0.90$ & 0.88 & $0.80-0.93$ \\
FAS category & 0.78 & $0.69-0.85$ & 0.73 & $0.57-0.84$ & 0.84 & $0.72-0.91$ & 0.81 & $0.67-0.89$ & 0.68 & $0.50-0.80$ \\
\hline
\end{tabular}

$\mathrm{N} / \mathrm{A}=$ Not applicable due to lack of variance.

\subsubsection{Completion rate}

In general, all items measuring SES in our study have a completion rate higher than $97 \%$. Out of 5876 pupils, the first and second lowest completion rate were items for parental education (father $97.9 \%$ and mother $98.4 \%$ ) and all the four items of FAS II have the same highest completion rate $(99.7 \%)$, which made the completion rate of the composite FAS scores $99.2 \%$. However, no significant differences were found between the completion rates of the four FAS II items 
and two parental education items. We also examined the completion rates by gender and by age groups. There is no difference in the completion rates of those SES items by gender except for the question asking about the family holidays $(p=0.042)$ which showed that boys were slightly less likely to report their family holidays than girls. Significant differences of completion rates were found in the three age groups for the item about asking about father's education $(p<0.001)$ and mother's education $(p<0.001)$, which indicated that younger children were less likely to report their parental educational level.

\subsubsection{Internal reliability}

As seen in Table 9, the internal consistency of FAS II items was examined by Cronbach's alpha coefficient and the value was 0.58 . The alpha coefficient changed if a single item was deleted from the scale. The item-rest coefficients suggested that deleting either family car $(\alpha=0.47)$, or family holidays $(\alpha=0.49)$, or numbers of computer $(\alpha=0.43)$ resulted in a drop of internal consistency. On the contrary, if own bedroom was deleted from the FAS II items, the alpha coefficient would improve to 0.60 . Higher internal consistencies were observed in older respondents.

The results of the inter-correlations between the FAS II items and the composite FAS score showed moderate associations (more than 0.60) except for the item asking about own bedroom (0.34) (Table 10). Rather poor correlations were found within the FAS II items (from 0.16 to 0.39 ) and the poorest one was found between items asking about own bedroom and family car (0.16), but all items were statistically intercorrelated $(p<0.01)$.

The Confirmatory factor analyses (CFA) showed that the comparative fit index (CFI) of 0.99 and the weighted root mean square residual of 1.10. The standardised factor loading ranged between 0.49 (number of own bedroom) and 0.69 (number of computers).

TABLE 9 Cronbach's alpha coefficient for FAS II items by gender and age in Chinese HBSC linked project survey $2008(\mathrm{~N}=5876)$.

\begin{tabular}{lcccccc}
\hline & \multicolumn{5}{c}{ Cronbach's alpha coefficient } \\
\cline { 2 - 7 } & Total & Boys & Girls & 11yrs & 13yrs & 15yrs \\
\hline All four items & 0.58 & 0.59 & 0.57 & 0.56 & 0.56 & 0.62 \\
Delete family car & 0.47 & 0.49 & 0.45 & 0.48 & 0.46 & 0.49 \\
Delete own bedroom & 0.60 & 0.61 & 0.59 & 0.55 & 0.57 & 0.66 \\
Delete family holiday & 0.49 & 0.50 & 0.48 & 0.49 & 0.45 & 0.53 \\
Delete No. of computers & 0.43 & 0.43 & 0.42 & 0.40 & 0.42 & 0.47 \\
\hline
\end{tabular}


TABLE 10 Spearman correlations of FAS II items in Chinese HBSC linked project survey $2008(\mathrm{~N}=5876)$.

\begin{tabular}{lcccc}
\hline & Family car & Own bedroom & Family holidays & No. of computers \\
\hline Own bedroom & $0.16^{* *}$ & & & \\
Family vacations & $0.32^{* *}$ & $0.22^{* *}$ & & \\
No. of computers & $0.39^{* *}$ & $0.20^{* *}$ & $0.36^{* *}$ & \\
FAS score & $0.67^{* *}$ & $0.34^{* *}$ & $0.69^{* *}$ & $0.69^{* *}$ \\
\hline
\end{tabular}

** $p<0.01$

\subsubsection{Validity}

The external validity of FAS II was evaluated by examining associations between FAS II and other SES indicators, using Spearman rank correlation $\left(r_{s}\right)$, univariate and multivariate ordinal logistic regression analyses. Statistically significant associations were found between the composite FAS II scores with father's education $\left(r_{s}=0.49, p<0.001\right)$, mother's education $\left(r_{s}=0.48, p<0.001\right)$, and perceived family wealth $\left(r_{s}=0.51, p<0.001\right)$.

Table 11 showed the distribution of all variables used in this study by FAS category and the unadjusted and adjusted odds ratios for all independent variables, with FAS low as the reference group. Students in rural areas were more likely to be located in the low FAS group. The percentage of respondents in the high FAS group increased if they reported their father or mother had a higher educational background. Similarly, the percentage of students in the high FAS group increased with their perceived family wealth. Both models revealed the same significant association between FAS II category and all independent variables (Table 11). Analyses by gender and age group showed that girls and elder students were more likely to report less family affluence, compared to boys and young students. Similar findings were also established for students who lived in rural areas or studied in a school from the district with poor economic status. The multivariate analyses indicated that students whose fathers' educational level was low and middle were respectively around 3 times and 1.7 times more likely to report less affluence than students whose fathers' educational level were high. Similar results were also observed for respondents with low and middle level educational mothers, where the OR's were about 2 and 1.6 respectively. The most prominent finding was that students whose perceived family wealth was low were 10 times more likely to report their family affluence was also low, compared to those who perceived their family wealth as high. 
TABLE 11 The distribution of independent variables by FAS category and ordinal regression results in Chinese HBSC linked project survey 2008, FAS low as the referent $(\mathrm{N}=$ 5876).

\begin{tabular}{|c|c|c|c|c|c|c|c|c|c|c|c|}
\hline & \multirow{2}{*}{$\frac{\text { Total }}{\mathrm{n}}$} & \multicolumn{2}{|c|}{$\begin{array}{l}\text { FAS } \\
\text { (low) }\end{array}$} & \multicolumn{2}{|c|}{$\begin{array}{l}\text { FAS } \\
\text { (middle) }\end{array}$} & \multicolumn{2}{|c|}{$\begin{array}{l}\text { FAS } \\
\text { (high) }\end{array}$} & \multicolumn{2}{|c|}{ Univariate OLR } & \multicolumn{2}{|c|}{ Multivariate OLR } \\
\hline & & $\mathrm{n}$ & $\%$ & $\mathrm{n}$ & $\%$ & $\mathrm{n}$ & $\%$ & OR & $95 \% \mathrm{CI}$ & OR & $95 \% \mathrm{CI}$ \\
\hline Overall & 5876 & 2421 & 41.2 & 2178 & 37.1 & 1229 & 20.9 & & & & \\
\hline \multicolumn{12}{|l|}{ Gender } \\
\hline Boys & 2816 & 1116 & 39.6 & 1015 & 36 & 655 & 23.3 & $0.84^{* * *}$ & $0.77-0.93$ & $0.82^{* *}$ & $0.79-0.88$ \\
\hline Girls & 3060 & 1305 & 42.6 & 1163 & 38 & 574 & 18.8 & 1 & & 1 & \\
\hline \multicolumn{12}{|l|}{ Age } \\
\hline 11 & 1941 & 624 & 32.2 & 776 & 40 & 525 & 27 & $0.48^{* * *}$ & $0.43-0.54$ & $0.54^{\star * *}$ & $0.48-0.62$ \\
\hline 13 & 1930 & 779 & 40.4 & 753 & 39 & 373 & 19.3 & $0.70^{* * *}$ & $0.63-0.79$ & $0.67^{* * *}$ & $0.59-0.77$ \\
\hline 15 & 2005 & 1018 & 50.8 & 649 & 32.4 & 331 & 16.5 & 1 & & 1 & \\
\hline \multicolumn{12}{|l|}{ Area } \\
\hline Urban & 2833 & 833 & 29.4 & 1136 & 40.1 & 843 & 29.8 & $0.37^{* * *}$ & $0.33-0.40$ & $0.75^{* * *}$ & $0.64-0.84$ \\
\hline Rural & 3043 & 1588 & 52.2 & 1042 & 34.2 & 386 & 12.7 & 1 & & 1 & \\
\hline \multicolumn{12}{|l|}{$\begin{array}{l}\text { School } \\
\text { location }\end{array}$} \\
\hline Good & 1943 & 626 & 32.2 & 750 & 38.6 & 555 & 28.6 & $0.39^{* * *}$ & $0.35-0.44$ & $0.57^{* * *}$ & $0.49-0.66$ \\
\hline Fair & 1956 & 731 & 37.4 & 798 & 40.8 & 409 & 20.9 & $0.53^{* * *}$ & $0.47-0.60$ & $0.62^{* * *}$ & $0.54-0.72$ \\
\hline Poor & 1977 & 1064 & 53.8 & 630 & 31.9 & 265 & 13.4 & 1 & & 1 & \\
\hline \multicolumn{12}{|c|}{$\begin{array}{l}\text { Father's } \\
\text { education }\end{array}$} \\
\hline Low & 2017 & 1329 & 65.9 & 539 & 26.7 & 130 & 6.4 & $11.87^{* * *}$ & $10.29-13.71$ & $3.05^{* * *}$ & $2.46-3.78$ \\
\hline Middle & 2278 & 845 & 37.1 & 968 & 42.5 & 446 & 19.6 & $3.48^{* * *}$ & $3.06-3.96$ & $1.66^{* * *}$ & $1.40-1.95$ \\
\hline High & 1456 & 187 & 12.8 & 624 & 42.9 & 637 & 43.8 & 1 & & 1 & \\
\hline \multicolumn{12}{|l|}{$\begin{array}{l}\text { Mother's } \\
\text { education }\end{array}$} \\
\hline Low & 2154 & 1384 & 64.3 & 600 & 27.9 & 146 & 6.8 & $11.31^{* * *}$ & $9.80-13.07$ & $2.54^{* * *}$ & $2.05-3.15$ \\
\hline Middle & 2301 & 818 & 35.5 & 999 & 43.4 & 470 & 20.4 & $3.34^{\star * *}$ & $2.93-3.81$ & $1.55^{\star * *}$ & $1.31-1.83$ \\
\hline High & 1327 & 177 & 13.3 & 545 & 41.1 & 597 & 45 & 1 & & 1 & \\
\hline \multicolumn{12}{|l|}{$\begin{array}{l}\text { Perceive } \\
\text { family } \\
\text { wealth }\end{array}$} \\
\hline Low & 752 & 605 & 80.5 & 113 & 15 & 27 & 3.6 & $21.43^{* * *}$ & $17.44-26.34$ & $10.51^{* * *}$ & $8.44-13.12$ \\
\hline Middle & 2918 & 1444 & 49.5 & 1126 & 38.6 & 325 & 11.1 & $5.02^{* * *}$ & $4.49-5.62$ & $3.25^{* * *}$ & $2.88-3.67$ \\
\hline High & 2185 & 369 & 16.9 & 930 & 42.6 & 870 & 39.8 & 1 & & 1 & \\
\hline
\end{tabular}


To highlight the construct validity of the indicators, the GLLRM model fitting item responses for our data is shown in Figure 2. Two coefficients were significant revealing LD (Local Dependence) between items; number of cars-number of computers $\left(\gamma^{p}=0.15\right)$ and number of cars-number of holidays $\left(\gamma^{p}=-0.13\right)$, which meant that there were correlation between the number of cars and number of computers, as well as the number of cars and number of holidays. However, these correlations are weak. The DIF (Differential Item Functioning) was observed between age and three items, 'own bedroom', 'number of holidays', and 'number of computers'. The results indicate that the chances of having own bedroom $\left(\gamma^{p}=0.41\right)$ and number of computers $\left(\gamma^{p}=0.16\right)$ increased with age, whereas the number of holidays $\left(\gamma^{p}=-0.28\right)$ decreased with age. The DIF was not found between gender and FAS II items.

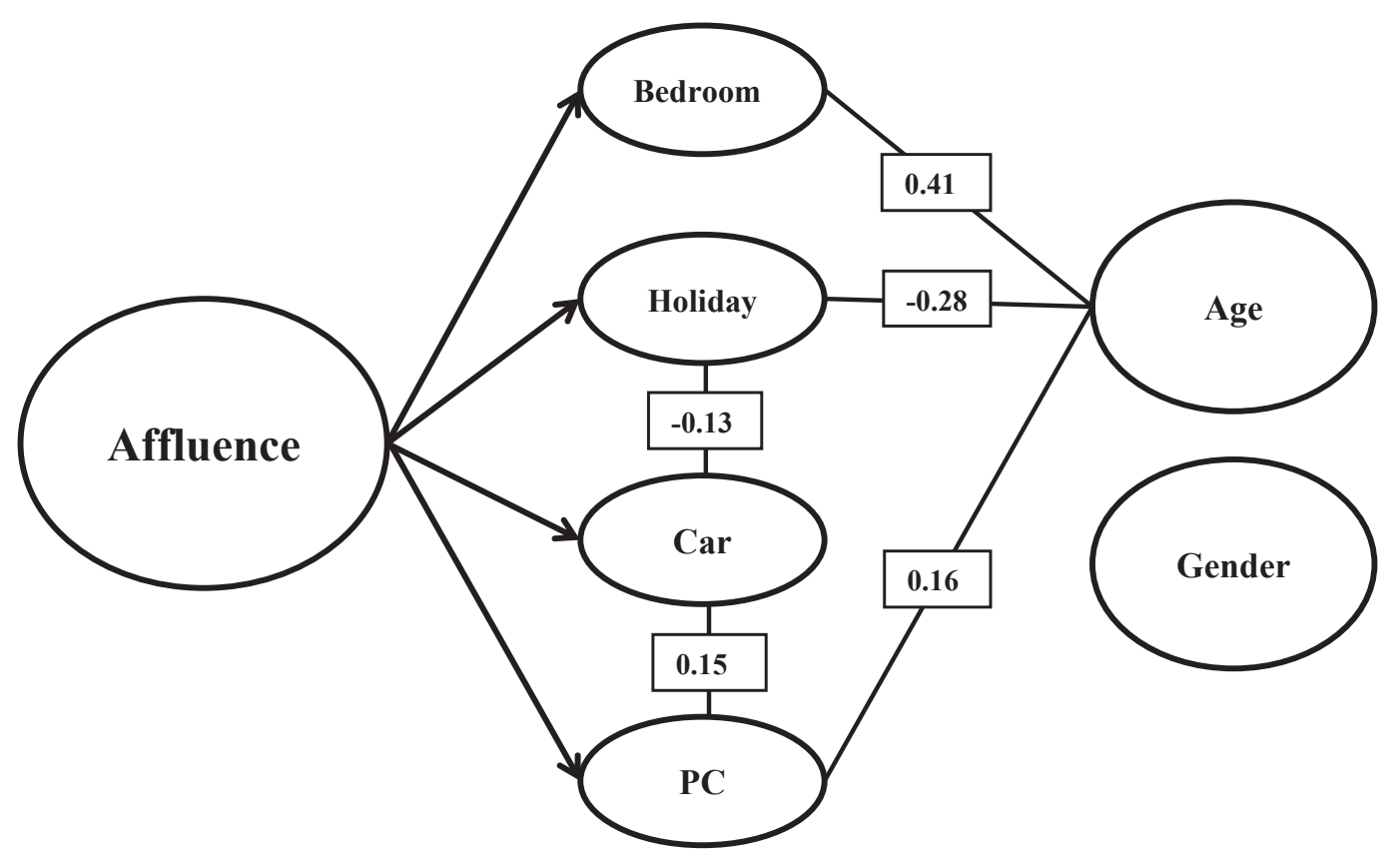

FIGURE 2 The generalization of the FAS II to GLLRM (numbers in boxes are partial gamma coefficients, $\mathrm{Y}^{p}$, indicate a significant correlation beyond the latent trait) in Chinese HBSC linked project survey $2008(\mathrm{~N}=5876)$. 


\subsection{Alcohol use and socioeconomic differences (Study III)}

In this article, the results were gained from 15 year-old students from China and Finland to demonstrate the prevalence of alcohol use of adolescents and explore the association between family affluence and alcohol use of adolescents.

The percentage of adolescents' alcohol use by gender and the FAS groups are shown in Table 12. Overall, the percentage of monthly alcohol use of Chinese 15 -year-old students is $18.7 \%$ while the percentage of Finnish adolescents' is approximately double (39.2\%), of which both Finnish boys and girls reported monthly alcohol use more often than Chinese boys and girls $(p<0.001)$. For the onset of alcohol use, nearly one quarter of Chinese adolescents and more than one third of Finnish adolescents reported that they had drunk alcohol at age 13 or younger. No significant difference was found for early onset of alcohol use between Chinese and Finnish boys. However, the results indicate that more Finnish girls (32.3\%) have early onset of alcohol use than Chinese girls $(20.5 \%)$ $(p<0.001)$. Similar to the results for alcohol consumption, Finnish respondents reported nearly five times greater drunkenness and three times greater early onset of drunkenness than Chinese respondents. The gender difference is clear among Chinese adolescents; more boys experienced monthly alcohol use, drunkenness and early onset of alcohol use and drunkenness than girls $(p<$ 0.001). However, this situation cannot be found among Finnish students. In general, the results reveal that, except for early onset of alcohol use among boys, the four measures of alcohol use have statistically significant differences among Chinese and Finnish adolescents $(p<0.001)$.

The percentages for alcohol use in different FAS groups are also shown in Table 12. The significant differences among the different FAS group were found among Chinese schoolchildren from Beijing except for being drunkenness, which was contrarily the only significant difference which was found among Finnish adolescents $(p=0.035)$. Compared to the adolescents in the low FAS group, Chinese respondents from Beijing who are in the middle and high FAS groups reported higher likelihood of alcohol use, and the percentages of alcohol use increased in line with the increasing of family affluence among Chinese students from Beijing. Different results were observed among Finnish students: the highest proportion of alcohol use can be found in the low FAS group for early onset of alcohol use and in the middle FAS groups for the other indicators of alcohol use. 
TABLE 12 Percentages of adolescents' alcohol use by country, gender and FAS groups in Chinese HBSC linked project survey $2008(n=2005)$ and Finnish HBSC survey 2006( $\mathrm{n}=1685)$.

\begin{tabular}{|c|c|c|c|c|c|c|c|c|}
\hline & \multicolumn{2}{|c|}{$\begin{array}{l}\text { Monthly } \\
\text { alcohol use }\end{array}$} & \multicolumn{2}{|c|}{$\begin{array}{l}\text { Early onset of } \\
\text { alcohol use }\end{array}$} & \multicolumn{2}{|c|}{ Drunkenness } & \multicolumn{2}{|c|}{$\begin{array}{l}\text { Early onset of } \\
\text { drunkenness }\end{array}$} \\
\hline & $\mathrm{n}$ & $\%$ & $\mathrm{n}$ & $\%$ & $\mathrm{n}$ & $\%$ & $\mathrm{n}$ & $\%$ \\
\hline \multicolumn{9}{|l|}{ China } \\
\hline Total & 373 & 18.7 & 492 & 24.5 & 206 & 10.3 & 153 & 7.6 \\
\hline \multicolumn{9}{|l|}{ Gender } \\
\hline Boys & 237 & 26.7 & 265 & 29.9 & 139 & 15.7 & 91 & 10.4 \\
\hline Girls & 136 & 12.2 & 227 & 20.5 & 67 & 6.1 & 62 & 5.6 \\
\hline$p$ & \multicolumn{2}{|c|}{$<0.001$} & \multicolumn{2}{|c|}{$<0.001$} & \multicolumn{2}{|c|}{$<0.001$} & \multicolumn{2}{|c|}{$<0.001$} \\
\hline \multicolumn{9}{|l|}{ FAS } \\
\hline Low & 156 & 15.4 & 225 & 22.2 & 94 & 9.3 & 67 & 6.7 \\
\hline Middle & 135 & 20.9 & 166 & 25.6 & 64 & 9.9 & 48 & 7.4 \\
\hline High & 80 & 24.2 & 100 & 30.6 & 46 & 13.9 & 37 & 11.5 \\
\hline$p$ & \multicolumn{2}{|c|}{$<0.001$} & \multicolumn{2}{|c|}{0.008} & \multicolumn{2}{|c|}{0.052} & \multicolumn{2}{|c|}{0.019} \\
\hline \multicolumn{9}{|l|}{ Finland } \\
\hline Total & 659 & 39.2 & 528 & 31.3 & 763 & 45.3 & 372 & 22.1 \\
\hline \multicolumn{9}{|l|}{ Gender } \\
\hline Boy & 317 & 40.2 & 242 & 31.7 & 367 & 46.6 & 179 & 22.5 \\
\hline Girl & 342 & 38.2 & 286 & 32.3 & 396 & 44.3 & 193 & 21.8 \\
\hline$p$ & \multicolumn{2}{|c|}{0.398} & \multicolumn{2}{|c|}{0.795} & \multicolumn{2}{|c|}{0.360} & \multicolumn{2}{|c|}{0.431} \\
\hline \multicolumn{9}{|l|}{ FAS } \\
\hline Low & 79 & 35.7 & 73 & 33.3 & 96 & 43.4 & 51 & 23.4 \\
\hline Middle & 327 & 41.3 & 256 & 33.1 & 386 & 48.8 & 191 & 24.6 \\
\hline High & 245 & 38.9 & 192 & 31.0 & 265 & 42.1 & 125 & 20.3 \\
\hline$p$ & \multicolumn{2}{|c|}{0.294} & \multicolumn{2}{|c|}{0.668} & \multicolumn{2}{|c|}{0.035} & \multicolumn{2}{|c|}{0.149} \\
\hline
\end{tabular}

Further, binary logistic regression models were executed to investigate the associations between all variables of alcohol use and FAS category stratified by gender for China and Finland and odds ratios with 95\% CIs are shown in Table 13. When the low FAS group was used as a reference group, Chinese girl respondents who belong to the high FAS group are more likely to report experiencing monthly alcohol use $(\mathrm{OR}=2.13)$ and early onset of alcohol use $(\mathrm{OR}=$ 1.55). Meanwhile, the Chinese boys categorised in the high FAS group have a risk of reporting more early onset of drunkenness $(\mathrm{OR}=1.92)$ compared to those who are in the low FAS group. However, the regression analyses revealed that, compared to the Chinese results, no significantly lower or higher odds ratios were found among Finnish boys and girls in both the middle and the high FAS groups. 
The associations between alcohol use and Family Affluence Scale by gender and country, odds ratios with $95 \%$ confidence intervals in Chinese HBSC linked project survey $2008(n=2005)$ and Finnish HBSC survey $2006(n=1685)$.

\begin{tabular}{|c|c|c|c|c|c|c|c|c|}
\hline & \multicolumn{4}{|c|}{ China } & \multicolumn{4}{|c|}{ Finland } \\
\hline & \multicolumn{2}{|c|}{ FAS Middle } & \multicolumn{2}{|c|}{ FAS High } & \multicolumn{2}{|c|}{ FAS Middle } & \multicolumn{2}{|c|}{ FAS High } \\
\hline & OR & $95 \% \mathrm{CI}$ & OR & $95 \% \mathrm{CI}$ & OR & $95 \% \mathrm{CI}$ & OR & $95 \% \mathrm{CI}$ \\
\hline \multicolumn{9}{|l|}{ Boys } \\
\hline Monthly alcohol use & 1.39 & 0.99-1.95 & 1.35 & $0.91-2.01$ & 1.51 & $0.96-2.37$ & 1.41 & $0.89-2.23$ \\
\hline Early onset of alcohol use & 1.05 & $0.75-1.46$ & 1.38 & $0.95-2.01$ & 0.78 & $0.49-1.23$ & 0.84 & $0.53-1.33$ \\
\hline Drunkenness & 1.03 & $0.67-1.57$ & 1.42 & $0.89-2.25$ & 1.22 & $0.79-1.89$ & 1.00 & $0.64-1.56$ \\
\hline Early onset of drunkenness & 1.01 & $0.60-1.70$ & 1.92 & $1.13-3.28$ & 0.99 & $0.61-1.65$ & 0.81 & $0.48-1.36$ \\
\hline \multicolumn{9}{|l|}{ Girls } \\
\hline Monthly alcohol use & 1.47 & $0.98-2.20$ & 2.13 & $1.31-3.47$ & 1.09 & $0.71-1.66$ & 0.94 & $0.61-1.46$ \\
\hline Early onset of alcohol use & 1.33 & $0.96-1.84$ & 1.55 & $1.02-2.35$ & 1.22 & $0.78-1.92$ & 0.97 & $0.61-1.55$ \\
\hline Drunkenness & 1.03 & $0.59-1.80$ & 1.39 & $0.70-2.76$ & 1.27 & $0.83-1.92$ & 0.90 & $0.58-1.39$ \\
\hline Early onset of drunkenness & 1.23 & $0.70-2.16$ & 1.30 & $0.62-2.72$ & 1.16 & $0.70-1.91$ & 0.86 & $0.50-1.46$ \\
\hline
\end{tabular}

Note: The reference group in logistic regression was adolescents in low FAS group. Statistically significant differences by gender or age group are in bold text $(p<0.05)$.

\subsection{Smoking and socioeconomic differences (Study IV)}

The last article moved its focus from alcohol use to smoking with the same data used in article III. The prevalence rates of smoking behaviour of Chinese and Finnish school-aged children are shown in Table 14.

In general, for all four variables of smoking behaviour, the results indicated that the prevalence is much higher among Finnish schoolchildren than Chinese schoolchildren $(p<0.001)$, especially demonstrated by the percentage of Finnish daily smokers being nearly seven times as many as the Chinese daily smoker, in that only $2.4 \%$ of Chinese pupils reported that they smoke every day. Nearly one fifth $(17.8 \%)$ of Chinese adolescents reported they had ever smoked and $11.6 \%$ of them smoked when they were 13 years or younger. This compares to the Finnish results which showed that more than half $(59.3 \%)$ of them have smoked, and $33.6 \%$ of them were classified as early smokers. Among Chinese respondents, gender difference was observed for all measures; boys exhibiting greater prevalence of smoking than girls $(p<0.001)$. In contrast, there were no differences of the percentage of ever smoked $(p=0.213)$ and being current smoker $(p=0.379)$ between Finnish boys and girls, although more boys were daily smoker $(p=0.027)$ or early smoker $(p=0.009)$ than girls.

The statistically significant differences of the rates of being a daily smoker among the different FAS groups were observed in both countries $(p<0.01)$ but with a different trend; the highest percentage of those being a daily smoker 
was found among Chinese respondents from the high FAS group, contrasting the results revealed among Finnish adolescents (Table 14).

TABLE 14 Percentages of adolescents' smoking by country, gender and FAS groups in Chinese HBSC linked project survey $2008(\mathrm{n}=2005)$ and Finnish HBSC survey $2006(\mathrm{n}=1685)$.

\begin{tabular}{|c|c|c|c|c|c|c|c|c|}
\hline & \multicolumn{2}{|c|}{$\begin{array}{c}\text { Ever } \\
\text { smoked }\end{array}$} & \multicolumn{2}{|c|}{$\begin{array}{l}\text { Daily } \\
\text { smoker }\end{array}$} & \multicolumn{2}{|c|}{$\begin{array}{l}\text { Current } \\
\text { smoker }\end{array}$} & \multicolumn{2}{|c|}{$\begin{array}{l}\text { Early onset } \\
\text { of smoking }\end{array}$} \\
\hline & $\mathrm{n}$ & $\%$ & $\bar{n}$ & $\%$ & $\bar{n}$ & $\%$ & $\mathrm{n}$ & $\%$ \\
\hline \multicolumn{9}{|l|}{ China } \\
\hline Total & 356 & 17.8 & 49 & 2.4 & 112 & 5.5 & 233 & 11.6 \\
\hline \multicolumn{9}{|l|}{ Gender } \\
\hline Boys & 265 & 29.7 & 49 & 5.5 & 102 & 11.5 & 175 & 19.6 \\
\hline Girls & 91 & 8.2 & 0 & 0 & 10 & 0.9 & 58 & 5.2 \\
\hline$p$ & \multicolumn{2}{|c|}{$<0.001$} & \multicolumn{2}{|c|}{$<0.001$} & \multicolumn{2}{|c|}{$<0.001$} & \multicolumn{2}{|c|}{$<0.001$} \\
\hline \multicolumn{9}{|l|}{ FAS } \\
\hline Low & 197 & 19.4 & 20 & 2.0 & 51 & 5.0 & 132 & 13.0 \\
\hline Middle & 97 & 14.9 & 12 & 1.8 & 36 & 5.5 & 59 & 9.1 \\
\hline High & 60 & 18.1 & 16 & 4.8 & 24 & 7.3 & 41 & 12.4 \\
\hline$p$ & \multicolumn{2}{|l|}{0.067} & \multicolumn{2}{|c|}{0.007} & \multicolumn{2}{|c|}{0.302} & \multicolumn{2}{|c|}{0.041} \\
\hline \multicolumn{9}{|l|}{ Finland } \\
\hline Total & 1000 & 59.3 & 280 & 16.6 & 499 & 29.5 & 586 & 33.6 \\
\hline \multicolumn{9}{|l|}{ Gender } \\
\hline Boys & 481 & 60.9 & 148 & 18.7 & 242 & 30.7 & 287 & 36.3 \\
\hline Girls & 519 & 58.0 & 132 & 14.7 & 257 & 28.7 & 279 & 31.2 \\
\hline$p$ & \multicolumn{2}{|c|}{0.213} & \multicolumn{2}{|c|}{0.027} & \multicolumn{2}{|c|}{0.379} & \multicolumn{2}{|c|}{0.009} \\
\hline \multicolumn{9}{|l|}{ FAS } \\
\hline Low & 134 & 60.4 & 48 & 21.6 & 67 & 30.2 & 83 & 37.4 \\
\hline Middle & 483 & 61.0 & 144 & 18.2 & 248 & 31.4 & 273 & 34.5 \\
\hline High & 365 & 57.8 & 82 & 13.0 & 175 & 27.8 & 199 & 31.5 \\
\hline$p$ & \multicolumn{2}{|l|}{0.460} & \multicolumn{2}{|c|}{0.003} & \multicolumn{2}{|c|}{0.340} & \multicolumn{2}{|c|}{0.179} \\
\hline
\end{tabular}

The logistic regression analyses of smoking and the FAS were performed in both countries stratified by gender (Table 15). When the smoking behaviour of students from the high FAS group was used as a reference group, we found that Chinese boys from the low (OR $=0.49,95 \%$ CI: 0.25-0.98) and the middle (OR = $0.43,95 \%$ CI: $0.20-0.92)$ FAS groups are less likely to be a daily smoker than those from high affluence families. In contrast, Finnish girls from the low (OR = 2.93, 95\% CI: 1.65-5.22) and the middle (OR $=1.99,95 \%$ CI: 1.27-3.12) FAS groups are more likely to report being a daily smoker compared to girls in the high FAS group. No other significant difference was found for the odds ratios of other variables of smoking. 
TABLE 15 The associations between smoking and Family Affluence Scale by gender and country, odds ratios with 95\% confidence intervals in Chinese HBSC linked project survey 2008 ( $\mathrm{n}=2005)$ and Finnish HBSC survey $2006(\mathrm{n}=1685)$.

\begin{tabular}{|c|c|c|c|c|c|c|c|c|}
\hline & \multicolumn{4}{|c|}{ China } & \multicolumn{4}{|c|}{ Finland } \\
\hline & \multicolumn{2}{|c|}{ FAS Low } & \multicolumn{2}{|c|}{ FAS Middle } & \multicolumn{2}{|c|}{ FAS Low } & \multicolumn{2}{|c|}{ FAS Middle } \\
\hline & $\mathrm{OR}$ & $95 \% \mathrm{CI}$ & OR & $95 \% \mathrm{CI}$ & OR & $95 \% \mathrm{CI}$ & OR & $95 \% \mathrm{CI}$ \\
\hline \multicolumn{9}{|l|}{ Boys } \\
\hline Ever smoked & 1.45 & $0.98-2.21$ & 0.84 & $0.55-1.29$ & 1.09 & $0.69-1.70$ & 1.15 & $0.84-1.58$ \\
\hline Current smoker & 0.81 & $0.47-1.38$ & 0.81 & $0.46-1.44$ & 0.91 & $0.56-1.47$ & 1.07 & $0.77-1.49$ \\
\hline Daily smoker & 0.49 & $0.25-0.98$ & 0.43 & $0.20-0.92$ & 1.24 & $0.71-2.15$ & 1.22 & $0.82-1.81$ \\
\hline Early onset of smoking & 1.46 & $0.93-2.29$ & 0.74 & $0.44-1.23$ & 1.31 & $0.83-2.06$ & 1.08 & $0.78-1.49$ \\
\hline \multicolumn{9}{|l|}{ Girls } \\
\hline Ever smoked & 1.01 & $0.53-1.90$ & 0.93 & $0.47-1.85$ & 1.13 & $0.73-1.75$ & 1.15 & $0.86-1.54$ \\
\hline Current smoker & 1.31 & $0.15-11.31$ & 1.75 & $0.19-15.79$ & 1.38 & $0.86-2.20$ & 1.33 & $0.96-1.83$ \\
\hline Daily smoker & $\mathrm{N} / \mathrm{A}$ & & $\mathrm{N} / \mathrm{A}$ & & 2.93 & $1.65-5.22$ & 1.99 & $1.27-3.12$ \\
\hline Early onset of smoking & 0.77 & $0.37-1.62$ & 0.76 & $0.34-1.70$ & 1.33 & $0.84-2.11$ & 1.28 & $0.94-1.75$ \\
\hline
\end{tabular}

Note: The reference group in logistic regression was adolescents in the high FAS group Statistically significant differences by gender or age group are in bold text $(p<0.05)$. N/A = Not applicable because of no daily smoker among girls. 


\section{DISCUSSION}

\subsection{Main research findings}

The purpose of this study was first to evaluate the feasibility of implementing the HBSC survey in a Chinese context and to examine the test-retest reliability of selected HBSC survey items. Secondly, this study investigated the prevalence of alcohol use and smoking of 15-year-old schoolchildren in China and Finland, and explored the associations between socioeconomic status and alcohol use and smoking. Comparison was made between these two countries to find the similarities and differences of socioeconomic inequalities in alcohol use and smoking of adolescents. Preceding the comparison, the validity of the FAS, serving as the main indicators of measuring SES, was also examined in a Chinese context. In accordance with the purpose, discussions on the research findings are presented.

\subsubsection{Feasibility of the HBSC survey}

There are various international or national studies aiming to monitor adolescent health and health behaviours conducted in China. However, the HBSC study has never been implemented, or even been piloted, in China before the present study. Organised by the Mass Sport Research Centre at the China Institute of Sport Science in collaboration with the Research Centre for Health Promotion at the University of Jyväskylä and Beijing Academy of Educational Sciences, the pilot study, and the Chinese HBSC linked project survey were conducted in 54 schools in the Beijing metropolitan area from October to December 2008. Overall, in accordance with the guidelines from the HBSC research protocol (Currie et al. 2001), the feasibility of implementing the HBSC survey in Beijing, China has been proved due to the high response rate and the proper length and content of the survey questionnaire.

The HBSC data for 2005/06 survey suggested that school/class and pupil level response rates exceeded $70 \%$ in the majority of participating countries (Robert et al. 2009). In the HBSC linked project survey in China, the response 
rate of sample schools is more than $90 \%$, which is higher than the response rate of sample schools in Finland. 5 out of 54 sample schools in Beijing did not participate in the HBSC linked project survey. Among those schools which dropped out, only one school presented the official refusal by the school principal due to the fact that there are items measuring sexual behaviours in survey questionnaire for 15-year-old pupils. The other four sample schools did not take part in the survey either because of the lack of enough sample classes and students, or due to the impossibility of implementing survey in the arranged period. The high response rate of sample schools demonstrated that the HBSC survey and survey content were accepted by almost all schools in Beijing, China.

In addition to the high response rate of schools, during the pilot study and later the HBSC linked project survey in Beijing, none of the sample classes and the students in the sample classes refused to fill in the questionnaire, which made the response rate higher than the response rate of Finnish pupils as well as the HBSC average, though the student could have refused to complete the survey since participation was voluntary or simply not fill-in the questionnaire if he or she did not want to answer it. Considering the rather high valid rate of respondents after data cleaning, this high response rate of sample students showed that Chinese schoolchildren did not express opposition to completion of the HBSC survey questionnaire.

According to the research protocols of the HBSC study (Currie et al. 2001), the questionnaire is self-explanatory so that it may reflect the student's own understanding of, and answers to the items. Thus, the administrator of the survey is not allowed to reply to the students' questions on interpreting the items/questions. In the pilot study, the researcher who was administrating the survey encouraged the students to ask any question concerning the items if they really did not understand the meaning or the explanation of the question. However, no questions were asked by the respondents. During the later large scale survey, there were very few cases of students asking about the meaning of items used in questionnaire. Therefore, conclusion can be drawn that those indicators measuring health behaviour as well as other contextual information in the HBSC survey questionnaire were proved to be understandable and acceptable to the school-aged children in Beijing.

Because the HBSC study is a school-based survey with data collection in classrooms, the length of the questionnaire and completion time of the questionnaire do also matter with regard to the feasibility of the study. The length of the survey questionnaire of the HBSC linked project in China varies for different age groups. The number of items is 104 for 11- and 13-year-old students, and 109 for 15-year-old students. Usually, students from the older group (13and 15-year-old) can finish the survey questionnaire in half an hour and it took a bit longer for 11 year-old students to complete. Nevertheless, all respondents could complete the questionnaire within one school hour (45 minutes) during the survey, which indicated that the questionnaire length is appropriate to use. 


\subsubsection{Test-retest reliability of selected HBSC survey items}

It is crucially important that the first step toward understanding young people's health behaviour is to obtain abundantly reliable and valid data. Surveys are the most common methodological technique to assess people's health behaviour, especially in epidemiological studies where the use of a self-report questionnaire is often the only feasible method of measurement (Kohl et al. 2000). Thus, the reliability of selected HBSC survey items was examined in the pilot study in Beijing, which included test and retest with a three week interval, prior to the later large scale HBSC linked project survey.

Overall, the test-retest reliability results showed moderate to almost perfect agreement for most of the items, except for one item about sedentary behaviour. Findings in our study suggest that these indicators are reliable to measure health behaviour of school-aged children in Beijing. A few gender and age group differences were observed in the reliability of some indicators measuring sedentary behaviour among respondents.

The reliability of items measuring physical activity in this study indicated that they are reliable measures, which is a similar finding compared to previous studies (e.g. Booth et al. 2001; Toreheim et al. 1997; Vuori et al. 2005). No gender and age group differences were found in this study, whereas some earlier studies have reported that the reliability of self-reported physical activity indicators generally improve with age (Booth et al. 2001; Rangul et al. 2008) and items about physical activity in the HBSC questionnaire were more reliable for girls (Rangul et al. 2008). A possible explanation for the non-existent difference within gender and age groups may be the fact that since 2007 the 'Sunshine Project' was carried out in all primary schools and high schools in China to ensure each student participates in physical activity at least one hour per day. This results in the students having clear understanding concerning physical activity participation so that the behaviour can be reported accurately no matter the age and gender. However, this conclusion should be viewed with caution since the sample size of this study is rather small.

Similarly to the earlier study of Hardy and his colleagues (2007), the items about sedentary behaviour in this study showed acceptable reliability. However, the reliability of items related to sedentary behaviour is lower than other behaviours. In general, the reasons for the low value of ICC are mainly due to poor reliability of answering the item, or the behaviour which the item measured is not very stable between the test and retest. The results also revealed a difference between age and gender groups, with younger students and girls tending to be more reliable than older students and boys for several items on sedentary behaviour. One exception that should be pointed out is for the item inquiring about 'playing computer or console games at weekends', which showed that boys are more reliable than girls probably because playing computer or console games is predominately a boys' activity, and girls' value of this activity is different, so that they might report inaccurately. 
Normally, for the self-report measures, the more response alternatives used, the more reliability is found. It is not surprising that at least substantial reliability was revealed in questions asking about sleeping habits since at least seven to fifteen response alternatives were recruited for them. Added to that, since sleep is a regular daily activity, knowledge and salience of sleep would be high. These results were very similar to the findings of Tynjälä's study (1999). It is evident for students that they have to wake up at a certain time in order to attend school on school days. Consequently, the items measuring sleeping behaviour are stable to some extent.

This study showed that items relating to alcohol use and smoking for 15year-old students have a good reliability, which is not surprising, as the finding is similar to previous studies on self-reported measures of adolescent drinking (Lintonen et al. 2004) and smoking (Henriksen \& Jackson 1999). An explanation for this is the fact that substance use displays a certain degree of cross-time stability, and therefore it can be recalled more reliably than other health behaviours (Tourangeau 2000). In addition, the salience of alcohol use and smoking might be higher compared to other health behaviours, since most students need an attitude towards such behaviours, like choosing whether they drink or smoke. Normally smoking behaviour would not change in the short term, but considering that student smoking is absolutely prohibited in Chinese schools and by most of their parents, it is understandable that the present smoking frequency of students may differ in terms of the different possibility to access cigarettes and smoke them over time. Another notable finding is that when students were asked about how often they drink beer, wine and strong liquors, the answers for wine and strong liquors are not as stable as for beer. The underlying reason for this is that many students have no clear definition of wine and strong liquors because compared to western countries, wine is rather seldom drunk for the masses in China, and the alcohol concentration of Chinese strong liquors varies a lot makes students' recall consumption unreliable compared to beer. Accordingly, these two items should be considered for revision or the addition of more reference explanations.

\subsubsection{Reliability and validity of FAS II}

One of the aims of this study is to investigate whether there are socioeconomic differences in adolescent drinking and smoking. To achieve such a goal, it is critical to employ reliable and valid indicators which measure socioeconomic status. Although FAS II, widely used in HBSC network countries in Europe and North America, was proved to be an easily answered and a useful indicator of child material affluence (Currie et al. 2008b), it should be examined whether FAS II is applicable before it is used in countries other than the current HBSC countries, such as China. In general, this study found that the FAS II items have high completion rates, an acceptable internal reliability, at least substantial testretest reliability, statistically significant associations with other SES indicators, only few LD, and weak DIF, which indicated that the reliability and validity of 
FAS II, as a SES measure of adolescents, are acceptable in the Beijing area (Liu et al. 2012).

Previous studies demonstrated the difficulties of obtaining information on parental education, occupation, and income, which are usually used as SES markers in adolescent studies, and it has been reported in many studies that there is a high non-response rate for those questions (Cho \& Khang 2010; Currie et al. 1997; Lin 2011; Molcho et al. 2007; Wardle et al. 2002). In contrast, it has been highlighted in previous studies that the FAS II items have much higher completion rates than other traditional SES indicators (Cho \& Khang 2010; Lin 2011; Molcho et al. 2007; Wardle et al. 2002). In our study, therefore, it is not surprising that a higher proportion of the respondents answered the four FAS II items than items on parents' highest educational level. However, the completion rates for parental education questions in our study were much higher than previous studies, although they revealed the lowest completion rates among all SES indicators analysed in the present study. One possible reason might be that students were instructed at the beginning of the survey to answer all the questions in sequence, which generated very low non-response rates for all survey questions. Consequently, no significant difference was found between the completion rates of the four FAS II items and the other SES indicators. When comparing the completion rates of the SES measures across gender and age, we found that there was no gender difference, which is similar to the results reported by Molcho and his colleagues (2007) except for the question asking about the family holidays. Age differences were found for items asking about parental education, indicating that younger children were less likely to report their parental educational level, again, similar results were also found by Molcho et al. (2007) and Lin (2011). We also found age differences of two FAS II items: family car and own bedroom, which are not consistent with other studies (Lin 2011; Molcho et al. 2007).

The test-retest reliability of FAS II has not been done and reported in earlier studies. As expected by Currie and her colleagues (2008b), the results from this first study analysing the test-retest reliability of the FAS II revealed that all four FAS II items, and the composite FAS score and category had at least substantial test-retest reliability due to the objectivity of the measures. In addition, the internal correlations between the FAS II items were in line with previous studies (Cho and Khang 2010; Lin 2011; Molcho et al. 2007; Schnohr et al. 2008). These results were understandable due to the limited range of the alternatives and each item describing a different aspect of family affluence (Wardle et al. 2002).

In present study, the value of Cronbach's alpha coefficient of FAS II items was found lower than 0.7 which is not regarded as satisfactory according to Bland and Alterman (1997). The first explanation is that these items measure different aspects of family affluent and may not have close internal consistency. In addition, the amount of FAS II items (only four) and their alternatives also contributes the rather lower value of Cronbach's alpha. Nevertheless, based on previous studies on internal consistency of FAS (Lin 2011) ( $\alpha=0.35)$ or home 
affluence scale (Wardle et al. 2002) $(\alpha=0.55)$, the internal consistency of FAS II items in Beijing, China ( $\alpha=0.58)$ was considered as acceptable.

Similar to earlier studies (Boudreau \& Poulin 2009; Cho \& Khang 2010; Lin 2011), this study found that the item on own bedroom had weaker correlations with the other FAS II items and the composite FAS score. In addition, the itemrest coefficients in our study concluded that if the item on own bedroom was deleted from the FAS scale, the alpha coefficient would improve, whereas deleting any other of the three items would reduce the internal consistency. Those findings suggested that further examination should be considered to choose the most sensitive items in specific settings since country and culture difference may affect the relative contribution of the four FAS II items to the composite FAS II (Schnohr et al. 2008). One extra item which may be considered and tested in the future is to ask the amount of weekly pocket money received by the student, which the child will reliably know the answer to, and is related to family affluence to some extent. Based on abovementioned results, the FAS II was demonstrated as a reliable measure of SES in a Chinese context.

The significant association between FAS II and other traditional SES measures, such as parental education and/or parental occupation (Lin 2011; Molcho et al. 2007; Richter et al. 2009) were found in previous studies, although the strength of associations varied in different countries. The rather low strength of association found in the present study was accounted for by the fact that FAS and parental education and occupation examine different aspects of socioeconomic status per se, and therefore may not overlap (Molcho et al. 2007; Wardle et al. 2002). Our study also revealed statistically significant associations between FAS and other SES indicators. The strength of associations in the present study were higher than previous findings, possibly indicating that the FAS II was related to other SES measures more closely in a Chinese context than others.

The external validity of FAS II was also assessed by ordinal logistic regression and the results were in line with the correlation analyses which confirmed the significant association between FAS II and other SES indicators. For instance, the students who perceived their family wealth as low were 10 times more likely to be in the low FAS category than those who perceived their family wealth as high, suggesting that the FAS II was more likely measuring the similar dimension of SES as perceived family wealth. This finding presented the possibility of adding the item on perceived family wealth as one item of the FAS scale for the development of the family affluences measure in the future. This is in line with Schnohr and her colleagues' (2008), who proposed that the FAS II can be used as a measure of wealth within a country and between countries, including the item on perceived family wealth. However, it should be pointed out that perceived family wealth is a subjective concept, and therefore it may not be comparable to measures of the FAS II which are more concrete in nature.

According to Rosenbaum (1989), two of the requirements of good construct validity are that items are mutually locally independent (no local dependence, LD) and conditionally independent of exogenic variables given the 
latent variable being measured (no differential item functioning, DIF). Thus, in present study, we further analysed the construct validity of FAS II through Graphical log-linear Rasch (GLLRM) model. The results indicated that the FAS II items are nearly locally independent and have very weak DIF, which can be considered as satisfied construct validity.

\subsubsection{Socioeconomic differences in alcohol use}

Regarding the alcohol use of adolescents, in general, this study found that monthly drinking and early onset of drinking is rather common in both China and Finland. Compared to China, many more Finnish schoolchildren have experienced drunkenness and early onset of drunkenness. Gender differences in alcohol use were hardly found among Finnish respondents whereas most of the drinking behaviour of Chinese youth was contributed by boys. Very interestingly, SES gradients of alcohol use were found among Chinese adolescents from Beijing, yet those gradients were absent among Finnish adolescents. In addition, some significant effects of family affluence on drinking and drunkenness were observed among Chinese schoolchildren but not among Finnish schoolchildren when analysed by gender.

The findings of the current study regarding socioeconomic differences in alcohol use of Chinese adolescents from Beijing are in line with previous studies conducted in developing countries. For instance, a recent study in Mexico indicated that more risk factors for current drinking were found in the middle or highest tercile of household expenditures compared with the lowest (Ritterman et al. 2009). This pattern was also revealed in a Brazilian study of adults, in which a higher SES was associated with higher rates of alcohol use (AlmeidaFilho et al. 2005). These differences support the diffusion of innovation theory that economic factor, as a determinant of adoption, would increase the diffusion process of drinking behaviour (Ferrence 2001). Therefore, those adolescents from well-off families are more likely to adopt the new behaviour (innovation), such as drinking alcoholic beverages or being drunk. However, the patterns of SES and drunkenness of Finnish adolescents reported in the present study are not consistent with Richter and his colleagues' study (2006) which was based on the results of the Finnish HBSC survey 2001/2002. This may be caused by the different SES measures used in these two studies, as well as the data being derived from different surveys.

The factors affecting the alcohol use in adolescents are complex, and there are several plausible interpretations for the differences of socioeconomic inequalities in alcohol use between Chinese and Finnish adolescents which were found by the present study. The first interpretation is that the adolescents' availability of resources to consume alcohol differs in China and Finland. Compared to the adult population, the accessibility of money for drinking is more limited for adolescents (Kunstche et al. 2004). For instance, a relationship between the amount of pocket money and drunkenness was found among Finnish adolescents (Kouvonen \& Lintonen 2002; Lintonen et al. 2000). There is a huge difference in national income and income inequality between China and Fin- 
land. China is a country with a rather high income inequality, and adolescents who are part of an affluent family usually have more pocket money and hence they have more possibility to buy alcohol than those in less affluent families. However, with regard to Finnish adolescents, although the amount of pocket money of young people in different FAS groups may still vary, even those who live in rather less affluent families might have enough money to buy alcoholic drinks due to the lower income inequality. Similarly, Elgar and his colleagues (2005) examined the effects of income inequality on drinking behaviour among 11,13 and 15-year-old students in 34 industrialized countries and found that income inequality was associated with drinking and drunkenness among younger adolescents but not among older adolescents.

Another possible explanation for the differences described above could be due to the different drinking culture between eastern and western countries, and when one considers whether adolescents may drink alcoholic beverages alone, or with their family or peers. Traditional Chinese cultural norms encourage social drinking (Cochrane et al. 2003), especially with family/relatives and friends. When Chinese schoolchildren were asked where they usually did their drinking, the answer was that the majority of them reported it to be at festivals when families get together (Newman 2002). It is understandable that Chinese adolescents in well-off families could have more opportunities to experience drinking at home, and therefore, drinking in a family situation may play an important role in affecting alcohol use of adolescents in China. In addition to the above-mentioned two explanations, the sensitivity of the FAS, as the indicator of socioeconomic differences in two countries, should be taken into account for the reasons of the absence of differences in Finland. Although the FAS has been shown as a reliable and valid SES measure in many HBSC member countries (Currie et al. 2008), as well as in Beijing, China (Liu et al. 2012), it still needs to be considered whether the socioeconomic inequalities using other socioeconomic measures can be detected in a country such as Finland, which has a developed social welfare system and rather small socioeconomic inequalities. The three categories of the FAS may not disclose the socioeconomic difference in the exact same way in Finland as in China.

\subsubsection{Socioeconomic differences in smoking}

With regard to the smoking of adolescents, this study found that the prevalence of different patterns of smoking behaviour is much higher among Finnish adolescents than Chinese adolescents from Beijing. Among Chinese respondents, gender difference was observed for all four measures of smoking, with boys exhibiting greater prevalence of smoking than girls. In contrast, there were no differences of the percentage of ever smoked and being a current smoker between Finnish boys and girls, although more boys were daily smokers or early smokers than girls. The findings also revealed different socioeconomic differences in being an adolescent daily smoker in these two countries, more specifically that Chinese boys from the low and the middle FAS group are less likely to be a daily smoker than those from high affluence families, and on the contra- 
ry, Finnish girls from the low and the middle FAS group are more likely to report being a daily smoker. No other significant difference was found for the socioeconomic difference among other indicators of smoking.

Although various results have been reported from previous studies concerning the socioeconomic inequalities in adolescents' smoking, a majority of studies found that daily smoking was most prevalent among students from lower socioeconomic groups (e.g. Rasmussen et al. 2009; Schnohr et al. 2009). However, it should be noted that those studies were mainly from western countries. Our findings regarding Chinese adolescents from Beijing indicate that boys from a less affluent family background are less likely to smoke daily, which is not consistent with the above-mentioned findings, but consistent with a recent Chinese study which reported that higher income relative to others is a risk factor for smoking among urban girls but not urban boys (Sun et al. 2012). Conversely, with regard to Finnish results, a different pattern was found, showing that female students from low and middle affluence families are at higher risk of being daily smokers. This result is in contrast to the study done by Paavola and her colleagues (2004), but is in line with a study which examined the socioeconomic differences in smoking among Finnish adolescents from 1977 to 2007, in which high rates of smoking were found in lower SES groups which persisted over time (Doku et al. 2010). However, it is noteworthy to point out that the inconsistent or consistent pattern of socioeconomic inequalities in adolescents' smoking revealed between present and previous studies should be viewed with caution due to the different measures of smoking and SES used, as well as different sampling strategies used in these studies which makes the results incomparable.

Smoking in adolescence is influenced by many factors. From the individual level, such as demographic factors (age, gender, ethnicity, SES), physiological differences (genetics, physiology reaction), and other risk behaviours, to the contextual level, such as peer relationships, parents and families' smoking, environmental/culture context (tobacco advertising and media message, taxation, cost and policies) (Turner et al. 2004). Thus, there are several plausible explanations for the different pattern of socioeconomic inequalities in daily smoking between Chinese and Finnish adolescents, as observed in our study. The first explanation is the adolescents' availability of resources to purchase tobacco products, since the amount of pocket money adolescents have has a strong relationship with current smoking (West et al. 2007). There is a huge difference in national income and income inequality between China and Finland. China is a country with a rather high income inequality, and adolescents who are part of an affluent family usually have more pocket money and hence they have more possibility to buy cigarettes for smoking than those in less affluent families. However, with regard to Finnish adolescents, although the amount of pocket money of young people in different FAS groups may still vary, even those who live in rather less affluent families might have enough money to buy cigarettes due to the lower income inequality. Moreover, it has been reported that socioeconomic position of the family had little impact on disposable money of teen- 
age boys and girls in Finland (Lintonen et al. 2007). Another possible explanation for the difference could be due to the different transition stages of smoking in China and Finland. It was reported that the prevalence of smoking has been falling since the 1970's in Nordic countries (Cavelaars et al. 2000). However, the smoking rates have not decreased simultaneously and equally in different socioeconomic groups. The rate of smoking among the higher social gradient has fallen more quickly, which leads to increasing inequalities of the smoking rate in different socioeconomic groups (Giskes et al. 2005).

Interestingly, no significant difference was found for the other three indicators of smoking behaviour measured in this study, including ever smoked, current smoker, and early onset of smoking among three FAS groups, which demonstrated that the socioeconomic inequalities may be neglected if we did not include daily smoker as the indicator of smoking. It has been argued that conclusions on socioeconomic inequality in adolescent smoking may appear differently when described by absolute and relative measures (Rasmussen et al. 2009). A certain smoking measure may be sensitive to a certain measure of SES, which also causes the fact that the inconsistent or even contradictory patterns of socioeconomic inequalities in previous studies may possibly be due to employing different measures of smoking and SES.

\subsection{Methodological considerations}

\subsubsection{Sampling}

This study was based on the analyses of the data from surveys using the HBSC research protocol. As one of the large scale international surveys, the specific population of the HBSC is young people attending school aged 11, 13, and 15. The first and foremost question is how to select these samples so that they can represent their nation/region. Although each country may choose to stratify their samples to ensure representation by, for instance, geography, ethnic group, and school type, the sample size recommended is approximately 1500 respondents among each age group to meet the requirement of a 95\% confidence interval and a design factor of 1.2 (Roberts et al. 2009). Another critical point concerning sampling is the mean age, which should be as close as possible to 11.5, 13.5 and 15.5 years. In order to get the desired mean age of the respondents, countries may consider when is the appropriate time to implement the survey in accordance with the specific school entry time for pupils in each country. In addition, cluster sampling is used in the HBSC study and the primary sampling unit is school class or school if the sampling frame of classes is absent.

Finland has taken part in the HBSC study ever since it started in 1983. The Finnish data used in this study (article III and IV) was from Finnish HBSC survey 2006 which followed the sampling procedure described by the HBSC research protocol. Schools all over Finland were selected using cluster sampling with the consideration of the size of the school. The sample class was randomly 
selected within the sample school. A response rate of more than $85 \%$ of schools and students showed that losses were very small (see Table 2 and 3). In addition, the valid rate of respondent was quite high. Therefore, the Finnish samples in this study may be regarded as representative of the whole country and generalizability of the results should not be a problem.

China has never conducted the HBSC survey before this study. Due to one of the purposes of this study being to examine the feasibility and reliability of the HBSC survey in a Chinese context as a pilot study for the further Chinese HBSC study, as well as the limited resource and financial support, the Chinese survey was conducted only in Beijing municipality. It should be pointed out that the health behaviour of adolescents in Beijing may not represent for the whole of China, especially for the rural areas of China if the different socioeconomic, geographic and cultural factors were taken into account.

In order to choose the most representative sample of Beijing, multi-stages stratification and random cluster sample design was used. Geographical location, socioeconomic development, as well as school conditions were considered as the factor to stratify the sample to ensure representation. Considering the mean age of the three age groups among the Finnish sample were 11.80, 13.78 and 15.78 years, the Chinese survey was conducted in December 2008 to ensure the similar mean age of Chinese samples in accordance with the school entry time for Chinese pupils. The response rates of sampled schools and students were very high, as well as the valid rate of respondents. Thus, the Chinese samples in this study may be regarded as representative of the Beijing area. Two months prior to the survey, the test-retest study was also carried out in two randomly drawn conventional sample schools in Beijing. Though one class dropped out in the retest, no significant difference of the characteristics was found in test between the dropped class and students participated in both test and retest. However, it might be argued that the sample size for the test-retest study is small and the two sampled schools both come from the urban area of Beijing.

\subsubsection{Comparability of data}

Another purpose of this study is the comparison of socioeconomic differences in alcohol use and smoking between Chinese and Finnish school-aged children. Tynjälä (1999) has argued that the matter of ensuring the comparability of data between countries is one critical point in a large scale survey with international comparisons, such as the HBSC study. Since the HBSC study relies on selfreport measures, question design is one of the most important issues regarding the comparability of data, due to the fact that questions are perceived in context, and that the different contexts (different countries and cultures) in which they exist may change the perception and the meaning of the questions (Harkness 2003). According to the conceptual framework of the HBSC study, the survey questionnaire comprises a range of health indicators and health behaviours of adolescents and their life circumstances. The indicators used in the current study for comparison purpose (article III and IV) include measurements of al- 
cohol use and smoking of adolescent, as well as the measurement of their family affluence. These questions, asking about specific behaviours like have you ever smoked, or drunk, or family affluence, are clearly defined and represent the same meaning in China and Finland, which may not be understood differently no matter what the different cultures are in these two countries. From the perspective of the question content, therefore, these indicators can be compared between China and Finland to some extent.

Another important issue concerning the comparability of data in international surveys is the translation of the questionnaire, which is a threat of causing inappropriate or in accurate understanding of the meaning of the questions. Smet et al. (1999) addressed that when adapted the HBSC in countries other than in Europe and North America, translation of questionnaire is one of threatens of the validity of this cross-cultural research. According to the translation guideline of the HBSC research protocol (Currie et al. 2001), national questionnaires should be translations and adaptations of the international standard version which is in English. Independent re-translation back to English and careful checking for discrepancies with the original English is required for all questionnaires. Both the Chinese and the Finnish survey questionnaire strictly followed the translation guideline and every effort has been made to ensure the identical meaning of questions as in the original English questionnaire. Thus, from the viewpoint of the translation, the questions involved in this study are comparable between Chinese and Finnish questionnaires.

In addition, the different time or season of implementing the survey should be taken into account when the study is aiming to compare the results from different countries. Health behaviours of school-aged children are changeable. For instance, behaviours like physical activity may vary between summer time and winter time due to the weather and environment circumstances. The data used in this study are from the Chinese survey conducted in October 2008, and the Finnish survey conducted from March to May 2006. Even through the time and weather were different of these two surveys, behaviours like alcohol use and smoking and family affluence analysed in this study might be stable and less influenced and therefore these two survey results can be compared.

It should be pointed out that one methodological limitation concerning the comparability in the present study is that the Chinese data were only sampled from the Beijing area due to the aims of the survey and the limited resources and financial support. In a country with huge diversity such as China, the results should be interpreted with caution since the sample cannot represent the whole nation. It is true that conclusions cannot be drawn based on the comparison of the prevalence of youth substance use. However, it is possible that the patterns of socioeconomic inequalities in alcohol use and smoking of adolescents in Beijing, as a reflection of such patterns in a Chinese population, can be compared with the patterns of other countries or regions. Nevertheless, the results of the comparison should be discussed and interpreted with caution. 


\subsubsection{Reliability and validity}

The HBSC study relies on self-report measures. Thus, reliability and validity of the indicators are essential. Previous studies addressing the reliability and validity of the HBSC survey questions have been done at national and international level. Most studies focused on items measuring certain behaviour(s) or factor(s), such as family affluence (Boyce et al. 2006), diet (Vereecken et al. 2003b), overweight and obesity (Elgar et al. 2005), physical activity (Booth et al. 2001; Rangul et al. 2008), symptoms (Haugland \& Wold 2001; Ravens-Sieberer et al. 2008) etc., except for Torsheim and his colleagues (1997) who investigated and evaluated more comprehensively the test-retest reliability of 31 selected items from the HBSC questionnaire. Findings from these studies indicate that most items of the HBSC survey questionnaire have acceptable reliability and validity.

To examine the reliability of the HBSC indicators in a Chinese population, this study represents the first assessment of test-retest reliability for selected HBSC survey questions (Liu et al. 2010). Overall, the findings suggest that HBSC survey items are reliable to measure health behaviour of school-aged children in Beijing. Particularly, analyses of indicators measuring alcohol use and smoking indicated at least moderate reliability. Furthermore, FAS II, used as a socioeconomic indicator in the present study, the reliability and validity were proved to be acceptable for adolescents in the Beijing area. The reliability of the HBSC survey items has also been examined among Finnish students (Ojala et al. 2005; Tynjälä 1999; Vuori et al. 2005) and the results revealed satisfactory test-retest reliability.

There are two methodological limitations regarding the reliability and validity in the present study. Firstly, the validity of indicators concerning substance use has still not been assessed in China and Finland. Even though the self-measurement of alcohol use and smoking, used in the HBSC survey, is considered as the most popular and only possible approach to assess substance use in a large population (Richter \& Johnson 2001), it should be noted that it usually underestimates the prevalence (Tourangeau \& Smith 1996; Turner et al. 1998) due to conscious attempts to provide inaccurate information for embarrassing or socially undesirable behaviours (Bradburn 1983). Secondly, the validity of FAS II was not examined in the Finnish population. Although a variety of crossnational studies have been done to explicitly validate different aspects of the FAS II (Boyce et al. 2006), which suggested that FAS is a valid measure of adolescent material affluence, the validity of FAS in a high-income and welfare state such as Finland has been contended since the FAS items were developed in the early 1990s and therefore they may not have enough power to distinguish different social gradients. The sensitivity of the FAS needs to be considered in that whether the socioeconomic inequalities can be detected in a country such as Finland, which has an organised social welfare system and rather small socioeconomic inequalities. The three categories of the FAS may not disclose the socioeconomic differences in the same way in Finland as in China. In addition, 
despite the fact that the FAS II items examine the material wealth including bedroom, computer, car and holidays, the culture meaning of these items presenting behind may differ in China and Finland.

\subsubsection{Data analyses and interpretation}

Reliability can be defined as the degree of consistency between two measures of the same thing (Mehrens \& Lehmann 1991). Statistically, the test-rest reliability can be measured by several approaches, including Pearson's Product-Moment Correlation Coefficient, Intraclass Correlation Coefficient (ICC) and Cohen's Kappa. Yen \& Lo (2002) summarized that three limitations of Pearson's Product-Moment Correlation should be taken into account when being used as a measure of test-retest reliability. Firstly, the purpose of the Pearson's ProductMoment Correlation is to determine the relationship between two variables, and theoretically it is not appropriate to apply the correlation to a case of two measures from the same variables. Secondly, the test-to-test variation is hardly to determine. The Pearson's Product-Moment Correlation cannot detect the existence of a systematic error. Cohen's Kappa can also be used to assess the testretest reliability of items with dichotomous alternatives. Most of the HBSC survey items have more than two alternatives, and after calculation, the ICC values were found to be identical with the Cohen's Kappa value for those items with dichotomised answers. Therefore, in article I and II of this study, the test-retest reliability of the HBSC survey items was estimated and represented by ICC. An important issue when using ICC method is to select an appropriate statistical model for a reliability study because of the six different forms the intraclass correlation described by Shrout \& Fleiss (1979). Considering the aim of test-retest reliability is measuring the extent of absolute agreement between two raters, the present study computed ICC through case 2 using a two-way random model with an absolute agreement type.

In article II, the internal consistency of FAS items was examined with Cronbach's alpha coefficient since it is the most commonly used estimation, based on the correlation among variables comprising a set. Moreover, for ordinal-level data, the Spearman rank correlation is one of the most common methods used to measure the direction and strength of the association between two variables. Thus, the external validity of FAS was assessed with Spearman rank correlation to examine the associations between FAS score with other SES indicators. In addition, ordinal logistic regressions were employed to demonstrate these associations. Kreiner \& Christensen (2011) pointed out that in behavioural research, the local dependence (LD) and differential item functioning (DIF) are common and Graphical Log Linear Rasch Models (GLLRM) are useful since they offer solutions to LD and DIF problems without eliminating items. Therefore, the construct validity of FAS items was also examined through GLLRM in this study.

When examining the associations between FAS and alcohol use and smoking of adolescents, logistic regression modelling was used in this study. Gender differences were observed in both countries, therefore, the analyses were done 
separated for boys and girls to avoid the confusion. However, this study did not include the mediating factors, such as family structure and school type, which may also influence the results. Another consideration is that the FAS was used as the only SES indicator due to the fact that other SES measures used in both China and Finland, like perceived family wealth and parents' employment status, showed extremely uneven distribution. In spite of the fact that FAS has a very high response rate in the present study, and it has been proved to be a reliable and valid measure of family wealth between countries (Schnohr et al. 2008), it is still a reflection of family material affluence which may not be absolutely consistent with the available resources that schoolchildren can use to buy alcoholic drinks or cigarettes. In addition, the dichotomous classification of alcohol use and smoking could also raise bias.

In addition, there are also several methodological limitations in the present study because of the study design of the HBSC survey. Firstly, the HBSC is a cross-sectional study. Patterns of distribution of health behaviours, such as alcohol use and smoking may fluctuate over time, with variable increases, decreases, or periods of stagnation across sections of a population. Secondly, the HBSC uses cluster sampling method, the basic unit is class or school, which may cause higher standard errors compared to a sampling of individuals. The data structure of the sample unit being school or class rather than student may generate larger confidence interval and therefore a misleading interpretation of the results, especially if the $p$ value is near 0.05 . The limitations of research methods discussed above need be considered when interpreting the results of the present study. Nevertheless, based on the HBSC research protocol, the current study provides the first comprehensive assessment of the test-retest reliability of the HBSC survey items in a Chinese context, and a unique comparison of socioeconomic differences in alcohol use and smoking between Chinese and Finnish school-aged children. 


\section{CONCLUSIONS AND IMPLICATIONS}

\subsection{Conclusions}

This study was the first to implement a survey using the HBSC research protocol in the mainland of China in order to examine the feasibility and reliability of the HBSC survey in a Chinese context, and compare the socioeconomic inequalities in alcohol use and smoking between Chinese and Finnish school-aged children. The main conclusions from this study are:

1. The implementing the HBSC survey in Beijing, China has been proven to be feasible, and selected HBSC survey items have satisfactory test-retest reliability for students in Beijing, China.

2. The HBSC FAS II is a reliable and valid SES measures for adolescents in Beijing, China.

3. Different patterns of socioeconomic inequalities in alcohol use between Chinese and Finnish adolescents were observed. Chinese students from more affluent families are more likely to report early onset of drunkenness for boys, and monthly drinking and early onset of drinking for girls, while the socioeconomic difference in alcohol use was absent among Finnish students.

4. Different patterns of socioeconomic inequalities in smoking between Chinese and Finnish adolescents were observed. Chinese boys from more affluent families are more likely to be daily smokers, while Finnish girls from less affluent families are more likely to be daily smokers. 


\subsection{Practical implications}

\subsubsection{Contribution to the HBSC linked project in China}

To the author's knowledge, this is the first study using the HBSC research protocol in mainland China where nearly 400 million school-aged children live. The process of the survey and research can be seen as a pilot study for the future Chinese HBSC linked project. The contributions of the present study to the HBSC linked project in China can be described as follows.

1. This study provided comprehensive information on the feasibility and reliability of the HBSC survey items in a Chinese context. It is important that the first step toward understanding young people's health behaviour is to obtain abundant and accurate data. For cross-national studies such as the HBSC study, the feasibility and reliability of survey instruments must be examined in the new joining country prior the HBSC survey. Meanwhile, the findings from China also contribute to the development of the HBSC study.

2. The process of implementing the survey in Beijing set a good example for future HBSC linked projects in China. Based on the HBSC research protocol, sampling framework and work guidelines have been designed and published during this study. Detailed information concerning research methods and how to implement the survey has been described. All these documents are valuable for the future large scale HBSC survey in China.

3. The current study initiated and enhanced the research collaboration between the Chinese and the Finnish HBSC research team. Comparison studies have been made benefiting considerably not only the scientific knowledge, but also in establishing the valued collaboration between countries. Moreover, young researchers who will devote themselves to continuing the future HBSC linked project, have been trained in the collaborative research.

Moreover, through the whole period of this study, the collaborative research team have made extensive research achievements, including research articles published in international and Chinese scientific journals, Masters' and doctoral theses, and many conference presentations. These research items made the Chinese HBSC linked project more notable on both national and international level.

Although progress and achievements have been made, the future challenges regarding the HBSC linked project in China still remain. Firstly, in order to implement the long term large scale research, financial support from multiple sources should be applied for to ensure the implementation of the study. Secondly, the research protocol as well as the survey items should be examined in 
a large population in different geographical areas in the future. Thirdly, the research team, especially the young researchers and professionals should be encouraged to take part in this study, to act as the human intelligence resource for the development of the future Chinese HBSC linked projects. Finally, national research networks should be built to organise and conduct the survey and manage the research project. Nevertheless, the CISS will be devoted to the HBSC linked project and aim to participate in the HBSC survey in 2013/14, in a large area of China.

\subsubsection{Reducing alcohol use and smoking of adolescents}

Reducing the prevalence of alcohol use and smoking of adolescents is one of the priorities in global public health. In general, to reduce the harm caused by substance use of adolescents, actions including national or regional policies, education and interventions have been made (Babor et al. 2010). However, resources aimed at reducing substance use are always limited. In order to achieve greater efforts, it is important to design effective policies or interventions targeting vulnerable populations. Previous studies found there are socioeconomic differences in adolescent alcohol use and smoking. Thus, one of the aims of this study was to investigate the patterns of socioeconomic inequalities in alcohol use and smoking of adolescents in China and Finland, so that suggestions concerning specific policies and interventions targeting different groups could be provided.

Findings from this study concerning Beijing, China revealed that students from the more affluent families are more likely to be involved in drinking and smoking, which suggested that when planning and implementing interventions/programmes aiming at reducing young people's drinking and smoking, the aims should be focused on those students from the more affluent families. This result may also suggest that in China, increasing the price of alcoholic drinks and cigarettes to control the affordability may not work as well since the young drinker or smoker from the more affluent families is not as sensitive to price as those from the less affluent families. In addition, gender differences of the prevalence of alcohol use and smoking suggested that in China, more attention should be paid to boys with regards to reducing their alcohol use and smoking.

Except for Finnish girls from the less affluent families, who are more likely to be daily smokers, this study did not find any socioeconomic difference in alcohol use and smoking of Finnish young people. In addition, this study found that alcohol use and smoking are common for both boys and girls in Finland with no significant difference between genders. Thus, findings from this study indicated that efforts should be made to reduce the currently high rates of drinking and smoking of adolescents in both genders, and all socioeconomic groups in Finland. Furthermore, the present study also suggests that the policy of increasing the minimum price of alcohol and cigarettes may play a greater role in reducing the prevalence of adolescent drinking and smoking in Finland than in China. 


\subsubsection{Future research challenges}

This study examined the feasibility of implementing the HBSC survey in China and the reliability of the items in the survey questionnaire, in a sample of schoolchildren in Beijing, China. Also, the study explored the associations between socioeconomic status and adolescents' alcohol use and smoking in China and Finland, and then compared the similarities and differences in these two countries.

Through the observation of students' participation in the survey and response rates of the sampled schools and sampled students, this study indicated that it is feasible to implement the HBSC survey in schools in Beijing, China. Although the absence of the inquiry on the survey questions and high response rates showed the feasibility objectively, the subjective acceptance of the survey should be investigated as well. Future research may employ a qualitative study on the feasibility, including interviews with students, teachers and school officials, to understand their perceived feeling on this survey.

This study provided a comprehensive examination of the test-retest reliability of selected HBSC survey items in a Chinese context. However, the sample size for the test-retest study was small and the two sampled schools both came from the urban area of Beijing. For a country like China, when the diversities of socioeconomic status and cultural background are taken into account, further similar research should be encouraged in different geographical areas among Chinese adolescents with a rather larger sample size. In addition, studies concerning the validation of the HBSC survey items should be conduct to ensure their validity in a Chinese context.

As one subjective indicator measuring SES in the HBSC study, FAS has been developed and widely used to address the association of SES with health behaviours (Currie et al. 2008a, b, 2012). Previous studies have shown that the FAS is valid in many European countries (e.g. Currie et al. 1997; Molcho et al. 2007). This study found that the FAS II is a reliable and valid measure of adolescents' SES in a Chinese context which added new knowledge to the literature. However, due to the limitations of this study as previously mentioned, the reliability and validity of the FAS should be evaluated in different places, especially in rural areas of China.

This study examined and compared the socioeconomic inequalities in alcohol use and smoking of adolescents among a European population and an Asian population. Different patterns of socioeconomic differences in adolescents' drinking and smoking were found in China and Finland. Research on socioeconomic inequalities in adolescent health behaviour between countries rather than within one country can provide evidence that socioeconomic status may not influence health behaviour independently of cultural and political contexts, and the differences presented in this study may reflect how different aspects of SES can influence one certain health behaviour in different countries. However, the factors related to alcohol use and smoking of adolescents are complex, and socioeconomic differences may be influenced by many variables. 
Future studies on socioeconomic inequalities' influence on alcohol use and smoking of adolescents may add these mediating factors as well to make more comprehensive and systematic analyses. Furthermore, the present study achieved information only from schoolchildren due to the study design of the HBSC survey. In future, surveys and/or interviews in school level, including teachers and headmasters, and family level, including parents or other guardians should be considered to understand the context of this phenomenon thoroughly. Finally, in order to understand when the socioeconomic differences establish and then define the important period for tackling inequalities, longitudinal study design should be preferred. 


\section{YHTEENVETO (FINNISH SUMMARY)}

Väestön terveyskäyttäytymiseen voidaan merkittävästi vaikuttaa nuoruusiässä. Nuoruus on kriittinen ikäkausi, jolloin monet terveystottumukset muotoutuvat ja ennustavat myös aikuisiän tottumuksia. Terveyden edistämisen tueksi tarvitaan luotettavaa tietoa nuorten terveyden ja terveystottumusten seuraamiseksi, ja samaa tutkimustietoa tarvitaan nuorten terveyttä edistävien interventioiden toteuttamiseksi ja arvioimiseksi. Verrattuna kehittyneisiin maihin vain hyvin harvassa tutkimuksessa kiinalaisten nuorten terveyskäyttäytymistä on kartoitettu laajemmin tai fokusoitu siten, että vertailu on ollut mahdollista. WHOKoululaistutkimus nuorten elintavoista (HBSC - Health Behaviour in Schoolaged Children Study) on käytössä jo yli 40 Euroopan ja Pohjois-Amerikan maassa. Tämä laaja tutkimusohjelma mahdollistaa nuorten koetun terveyden, terveyskäyttäytymisen ja elintapojen tutkimisen yhteisen kansainvälisesti käytetyn tutkimusprotokollan avulla. Jotta HBSC-tutkimuksen käyttökelpoisuus, reliaabelius ja validius kiinalaisessa kontekstissa voitaisiin varmistaa ja valmistautua tulevaan HBSC-projektiin Kiinassa, tämän väitöskirjatutkimuksen ensimmäisenä tavoitteena on ollut selvittää HBSC tutkimuksen toteutettavuutta Kiinassa sekä arvioida valikoitujen kyselyosioiden luotettavuutta kiinalaisessa kontekstissa.

Alkoholin käyttö ja tupakointi ovat riski nuorten kasvulle ja kehitykselle ja ne myötävaikuttavat monien terveydellisten ja sosiaalisten ongelmien syntyyn. Tämän vuoksi nuorten alkoholin käytön ja tupakoinnin vähentämistä pidetään globaalina prioriteettina kansanterveyden edistämisessä. Näin on myös Kiinassa ja Suomessa. Lisähaaste päätöksenteolle ja terveyden edistämiselle on se, miten rajallisia resursseja saadaan kohdennettua sellaisten tehokkaiden interventio-ohjelmien laatimiseen ja toteuttamiseen, jotka on suunnattu riskialttiille väestönosille. Aikaisemmissa tutkimuksissa on havaittu yhteyksiä nuorten sosioekonomisen taustan ja heidän terveyskäyttäytymisensä välillä. Tutkimustulokset, jotka liittyvät sosioekonomisen epätasa-arvon ja alkoholin käytön ja tupakoinnin välisiin yhteyksiin, ovat kuitenkin keskenään jossain määrin ristiriitaisia. Tähän tutkimustematiikkaan liittyvää vertailuaineistoa ei ole juurikaan käytettävissä sellaisista maista, joilla on huomattavan erilainen yhteiskunnallinen ja kulttuurinen tausta. Niinpä tämän väitöstutkimuksen toisena tavoitteena on ollut tutkia FAS (Family Affluence Scale - perheen varallisuusindeksi) mittarin avulla määriteltyjen sosioekonomisten indikaattorien ja nuorten alkoholin käytön ja tupakoinnin välisiä yhteyksiä kiinalaisilla ja suomalaisilla nuorilla. Tässä väitöskirjatyössä tutkittiin myös sosioekonomiseen epätasa-arvoon liittyviä eroja ja yhtäläisyyksiä näiden nuorten alkoholin käytössä ja tupakoinnissa.

Tämän tutkimuksen analyysit on tehty seuraavista kolmesta tutkimuksesta saatujen aineistojen perusteella: 1) testi-uusintatesti -tutkimus Pekingissä, Kiinassa, v. 2008 ( $\mathrm{n}=95$; kaksi kuudetta luokkaa, joiden oppilaat olivat n. 11vuotiaita sekä kaksi 10. luokkaa, joiden oppilaat olivat n. 15-vuotiaita; satunnaisotanta kahdesta satunnaisesta otoksessa olleesta koulusta); 2) kiinalainen 
HBSC-kyselyyn linkitetty tutkimus v. 2008 (n = 5876; monivaiheisen ositetun otannan ja satunnaisen ryväsotannan avulla valittiin 11-, 13- ja 15-vuotiaita nuoria Pekingistä, Kiinasta) sekä Suomessa tehty HBSC-koululaiskysely vuodelta 2006 (n = 5255; 11-, 13-, ja 15-vuotiaista nuorista koostuvat otokset kerättiin suomalaisista kouluista ryväsotannalla, jossa koulu poimittiin kyseisen luokkatason kokonaisoppilasmäärän suhteutettua todennäköisyyttä käyttäen (PPS). Kaikissa tutkimuksissa on käytetty HBSC-tutkimuksen protokollaa.

Tämän väitöskirjatutkimuksen ensimmäisessä vaiheessa valittujen HBSCkyselyosioiden testikertojen välisen toistettavuuden arviointi liittyi terveyskäyttäytymiseen (alkoholin käyttö, tupakointi, liikunta, liikunnallisesti passiivinen ajankäyttö, ja uni) ja se tehtiin määrittämällä mittausten välinen korrelaatio (Intraclass Correlation Coefficients, ICC) 95 nuoren otoksesta, johon kuului 11- tai 15-vuotiaita tyttöjä ja poikia Pekingistä, Kiinasta. FAS-mittarin reliaabeliutta ja validiutta tutkittiin Cronbachin alfa-kertoimen, Spearmanin korrelaatioiden, konfirmatorisen faktorianalyysin (CFA), järjestysasteikollisen regressioanalyysin sekä GLLRM (Graphic Log Linear Rasch Model) -mallin avulla 5876 koululaisen ryhmässä, joka oli mukana kiinalaisessa HBSC-kyselyyn liittyvässä tutkimuksessa. FAS-mittarin toistettavuusominaisuuden reliaabeliutta arvioitiin 95 koululaisen ryhmässä, joka osallistui testi-uusintatesti-tutkimukseen. Toisessa vaiheessa otokseen sisältyi 2005 kiinalaista ja 1685 suomalaista 15-vuotiasta nuorta. FAS-indikaattoreiden yhteyttä alkoholin käyttöön ja tupakointiin arvioitiin erikseen pojilla ja tytöillä kummankin sukupuolen osalta molemmissa maissa käyttämällä binaarista logistista regressioanalyysia.

Pekingissä Kiinassa vuonna 2008 toteutetussa testi-uusintatestitutkimuksessa, joka toimi myös pilottitutkimuksena, yksikään oppilaista ei kieltäytynyt osallistumasta kyselyyn ja kaikki vastasivat siihen annetussa ajassa. He eivät myöskään kysyneet mitään kyselyn osioista tai kertoneet opettajalleen, etteivät olisi ymmärtäneet kysymyksiä ja vastausvaihtoehtoja. Terveyskäyttäytymistä koskeviin osioihin liittyen vastausten pysyvyyden suhteelliset osuudet testin ja uusintatestin välillä vaihtelivat 32-92 \% välillä ja vähintään 68 \% vastaajista antoi vastauksensa joko samassa tai rinnakkaisessa vastauskategoriassa. ICC-korrelaatiokertoimet valittujen osioiden osalta vaihtelivat välillä 0,33-0,85. Kaikista 23 osiosta, joita arvioitiin tässä tutkimuksessa, 6 osion (26\%) reliaabelius oli kohtalainen, 12 osion (52\%) huomattava ja 4 osion (17 \%) lähes täydellinen.

FAS-mittareista tässä tutkimuksessa selvisi, että FAS II:n osioihin vastausprosentti on erittäin korkea (> 99 \%) ja parempi kuin muissa sosioekonomista statusta (SES) kuvaavissa mittareissa. Reliabiliteettianalyysit osoittivat kohtalaista sisäistä reliaabeliutta (Cronbachin alfa $=0,58$ ) ja huomattavaa toistettavuuden reliaabeliutta (ICC > 0,75). FAS II:n kohtalainen ulkoinen validius määrittyi Spearmanin korrelaatioanalyysin $\left(r_{s}=0,48-0,51, p<0,001\right)$ ja järjestysasteikollisen regressioanalyysin avulla tutkittaessa FAS II:n ja muiden SESindikaattoreiden (vanhempien koulutustaso ja käsitys perheen vauraudesta) välistä yhteyttä. GLLRM-analyysi puolestaan osoitti, että FAS-mittarin rakennevalidius on riittävän hyvä. 
Vertailututkimus alkoholin käytöstä osoitti, että Kiinassa, Pekingissä varallisuudeltaan ylemmästä FAS-ryhmästä tulevat nuoret kertoivat muita todennäköisemmin käyttävänsä alkoholia kuukausittain ( $\mathrm{OR}=2,13,95$ \% CI: 1,31$3,47)$ ja että tytöillä käyttö alkoi nuorempana (OR =1,55, $95 \%$ CI: 1,02-2,35) kun taas pojilla humalatilaan juominen alkoi nuorempana $(\mathrm{OR}=1,81,95 \% \mathrm{CI}=$ 1,13-3,28). Sen sijaan suomalaisten nuorten osalta kolmen FAS-ryhmän välillä ei ollut tilastollisesti merkitseviä eroja minkään neljän alkoholin käyttöä kuvaavan muuttujan välillä. Vertailututkimus tupakoinnista taas osoitti, että varallisuudeltaan alemmasta $(\mathrm{OR}=0,49,95 \% \mathrm{CI}$ : 0,25-0,98) ja keskimmäisestä FASryhmästä $(\mathrm{OR}=0,43,95 \% \mathrm{CI}: 0,20-0,92)$ tulevat kiinalaiset pojat ovat todennäköisesti vähemmän päivittäin tupakoivia. Sen sijaan varallisuudeltaan alemmasta (OR = 2,93, 95 \% CI: 1,65-5,22) ja keskimmäisestä FAS-ryhmästä (OR $=1,99,95 \%$ CI: 1,27-3,12) tulevat suomalaiset tytöt kertovat muita todennäköisemmin olevansa päivittäin tupakoivia. Kiinalaisten tyttöjen ja suomalaisten poikien osalta kolmen FAS-ryhmän ja kaikkien neljän tupakointia kuvaavan muuttujan välillä ei ollut tilastollisesti merkitseviä eroja.

Tämä tutkimus on ensimmäinen manner-Kiinassa tehty tutkimus, jossa käytettiin HBSC-tutkimusprotokollaa. Kyselyn ja tutkimuksen prosessi voidaan nähdä pilottina tulevalle HBSC-koululaistutkimusprojektille Kiinassa. Tulokset osoittivat, että HBSC-kysely on käyttökelpoinen Pekingissä, Kiinassa ja että valikoitujen HBSC-osioiden reliaabelius eri testikertojen välillä on tyydyttävällä tasolla myös pekingiläisessä koululaistutkimuksessa. Lisäksi tämä tutkimus on osoittanut, että HBSC FAS II on luotettava ja validi myös pekingiläisten nuorten sosioekonomisen taustan mittariksi. Tulokset antavat kattavaa tietoa HBSCkyselyn osioiden toimivuudesta ja luotettavuudesta kiinalaisessa kontekstissa, mikä edesauttaa tulevan HBSC-koululaistutkimuksen tekemistä Kiinassa.

Sosioekonomisen epätasa-arvon merkityksen vertaileminen suhteessa nuorten alkoholin käyttöön ja tupakointiin paljasti tiettyjä eroja tutkittujen kahden maan välillä. Niiden pohjalta voidaan päätellä, että suunniteltaessa ja toteutettaessa sellaisia interventioita ja ohjelmia, joiden tavoitteena on vähentää nuorten juomista ja tupakointia Kiinassa, kohderyhmänä pitäisi olla erityisesti varakkaammista perheistä tulevat nuoret. Sen sijaan Suomessa kohderyhmäksi pitäisi valita sekä tyttöjä että poikia kaikista sosioekonomisista luokista, kun pyritään vähentämään heidän alkoholinkäyttöään ja tupakointiaan.

Avainsanat: nuoret, alkoholin käyttö, perheen varallisuusindeksi, terveyskäyttäytyminen, tupakointi, sosioekonominen epätasa-arvo, WHO-

Koululaistutkimus, FAS-mittari 


\section{REFERENCES}

Aarø, L. E., Wold, B., Kannas, L. \& Rimpelä, M. 1986. Health behaviour in school-children A WHO cross-national survey: a presentation of philosophy, methods and selected results of the first survey. Health Promotion International 1 (1), 17-33.

Adams, J. \& White, M. 2009. Time perspective in socioeconomic inequalities in smoking and body mass index. Health Psychology 28 (1), 83-90.

Adler, N. E. \& Ostrove, J. M. 1999. Socioeconomic status and health: what we know and what we don't. Annals of the New York Academy of Sciences 896, 3-15.

Ahlström, S. \& Österberg, E. L. 2004. International perspectives on adolescent and young adult drinking. Alcohol Research \& Health 28 (4), 258-268.

Ahlström, S., Metso, L. \& Tuovinen, E. L. 2004. Suomalaisten nuorten päihteiden käyttö 1999 ja 2003. ESPAD-tutkimuksen taulukkoraportti. [Finnish young people's substance use in 1999 and 2003. The ESPAD report]. Aiheita 32/2004. Helsinki: STAKES.

Ahmad, S. \& Billimek, J. 2007. Limiting youth access to tobacco: Comparing the long-term health impacts of increasing cigarette excise taxes and raising the legal smoking age to 21 in the United States. Health Policy 80 (3), 378391.

Almeida-Filho, N., Lessa, I., Magalhaes, L., Araujo, M. J., Aquino, E., James, S. A. \& Kawachi, I. 2005. Social inequality and alcohol consumption-abuse in Bahia, Brazil: interactions of gender, ethnicity and social class. Social Psychiatry and Psychiatric Epidemiology 40, 214-222.

Andersen, A., Due, P., Holstein, B. E. \& Iversen, L. 2003. Tracking drinking behaviour from age 15-19 years. Addiction 98, 1505-1511.

Andersen, A., Krølner, R., Currie, C., Dallago, L., Due, P., Richter, M., Orkényi, A. \& Holstein, B. E. 2008. High agreement on family affluence between children's and parents' reports: international study of 11-year-old children. Journal of Epidemiology and Community Health 62 (12), 1092-1094.

Anderson Johnson, C., Palmer, P. H., Chou, C. P., Pang, Z., Zhou, D., Dong, L., Xiang, H., Yang, P., Xu, H., Wang, J., Fu, X., Guo, Q., Sun, P., Ma, H., Gallaher, P. E., Xie, B., Lee, L., Fang, T. \& Unger, J. B. 2006. Tobacco use among youth and adults in mainland China: The China Seven Cities Study. Public Health 120 (12):1156-1169.

Anderson, P. \& Baumberg, B. 2006. Alcohol in Europe: a public health perspective. A report for the European Commission. London: Institute of Alcohol Studies. 
Anderson, P., de Brujin, A., Angus, K., Gordon, R. \& Hastings, G. 2009. Impact of alcohol advertising and media exposure on adolescent alcohol use: a systematic review of longitudinal studies. Alcohol and Alcoholism 44 (3), 229-243.

Arria, A. M., Dohey, M. A., Mezzich, A. C., Bukstein, O. G. \& Van Thiel, D. H. 1995. Self-reported health problems and physical symptomatology in adolescent alcohol abusers. Journal of Adolescent Health 16 (3), 226-231.

Audrain-McGovern, J., Rodriguez, D., Tercyak, K. P., Cuevas, J., Rodgers, K. \& Patterson, F. 2004. Identifying and characterizing adolescent smoking trajectories. Cancer Epidemiology Biomarkers \& Prevention 13 (12), 20232034.

Babor, T. F., Caetano, R., Casswell, S., Edwards, G., Giesbrecht, N., Graham, K., Grube, J. W., Hill, L., Holder, H., Homel, R., Livingston, M., Österberg, E., Rehm, J., Room, R. \& Rossow, I. (Eds.) 2010. Alcohol: no ordinary commodity: research and public policy. $2^{\text {nd }}$ Edition. Oxford: Oxford University Press.

Bandura, A. 1986. Social foundations of thought and action. Englewood Cliffs, NJ: Prentice Hall.

Bangert-Drowns, R. L. 1988. The effects of school-based substance abuse education - a meta-analysis. Journal of Drug Education 18 (3), 243-264.

Barkin, S. L., Smith, K. S. \& DuRant, R. H. 2002. Social skills and attitudes associated with substance use behaviours among young people. Journal of Adolescent Health 30 (6), 448-454.

Beijing Municipal Bureau of Statistics. 2007. Beijing statistical year book 2007. Beijing: China Statistical Publishing House.

Beijing Municipal Bureau of Statistics. 2010. Beijing statistical year book 2010. Beijing: China Statistical Publishing House.

Beijing Municipal Commission of Education. 2007. List of comprehensive schools in Beijing. Unpublished.

Bewley, B. R. \& Bland, J. M. 1977. Academic-performance and social-factors related to cigarette smoking by schoolchildren. British Journal of Preventive \& Social Medicine 31 (1), 18-24.

Biglan, A., Duncan, T. E., Ary, D. V. \& Smolkowski, K. 1995. Peer and parental influences on adolescent tobacco use. Journal of Behavioural Medicine 18 (4), 315-330.

Bisset, S., Markham, W. A. \& Aveyard, P. 2007. School culture as an influencing factor on youth substance use. Journal of Epidemiology \& Community Health 61 (6), 485-490.

Björkqvist, K., Båtman, A. \& Aman-Back, S. 2004. Adolescents' use of tobacco and alcohol: correlations with habits of parents and friends. Psychological Reports 95 (2), 418-420. 
Bland, J. M. \& Alterman, D. G. 1997. Cronbach's alpha. British Medical Journal $314,572$.

Blank, M. D., Disharoon, S. \& Eissenberg, T. 2009. Comparison of methods for measurement of smoking behavior: Mouthpiece-based computerized devices versus direct observation. Nicotine \& Tobacco Research 11 (7), 896-903.

Bloomfield, K., Grittner, U., Kramer, S. \& Gmel, G. 2006. Social inequalities in alcohol consumption and alcohol related problems in the study countries of the EU Concerted Action 'Gender, Culture and Alcohol Problems: a multi-national study'. Alcohol and Alcoholism 41 (Supplement 1), i26-i36.

Booth, M. L., Okely, A. D., Chey, T. \& Bauman, A. 2001. The reliability and validity of the physical activity questions in the WHO health behaviour in schoolchildren (HBSC) survey: a population study. British Journal of Sports Medicine 35 (4), 263-267.

Botvin, G. J., Epstein, J. A., Schinke, S. P. \& Diaz, T. 1994. Predictors of cigarette smoking among inner-city minority youth. Journal of Development \& Behavioural Pediatrics 15 (2), 67-73.

Botvin, G. J., Griffin, K. W., Paul, E. \& Macaulay, A. P. 2003. Preventing tobacco and alcohol use among elementary school students through life skills training. Journal of Child \& Adolescent Substance Abuse 12 (4), 1-17.

Boudreau, B. \& Poulin, C. 2009. An examination of the validity of the Family Affluence Scale II (FAS II) in a general adolescent population of Canada. Social Indicators Research 94 (1), 29-42.

Boyce, W., Torsheim, T., Currie, C., \& Zambon, A. 2006. The Family Affluence Scale as a measure of national wealth: Validation of an adolescent selfreport measure. Social Indicators Research 78 (3), 473-487.

Bradburn, N. W. 1983. Response effects. In P. Rossi, J. Wright \& A. Anderson (Eds.) Handbook of survey research. New York: Wiley, 321-340.

Braveman, P. A., Cubbin, C., Egerter, S., Chideya, S., Marchi, K. S., Metzler, M. \& Posner, S. 2005. Socioeconomic status in health research: one size does not fit for all. The Journal of the American Medical Association 294 (22), 2879-2888.

Brener, N. D., Billy, J. O. G. \& Grady, W. R. 2003. Assessment of factors affecting the validity of self-reported health-risk behaviour among adolescents: evidence from the scientific literature. Journal of Adolescent Health 33, 436-457.

Brener, N. D., Kann, L., Kinchen, S., Grunbaum, J. A., Whalen, L., Eaton, D., Hawkins, J. \& Ross, J. G. 2004. Methodology of the Youth Risk Behavior Surveillance System. Morbidity and Mortality Weekly Report 53 (RR-12), $1-13$. 
Broms, U., Silventoinen, K., Madden, P. A., Heath, A. C. \& Kaprio, J. 2006. Genetic architecture of smoking behaviour: a study of Finnish adult twins. Twin Research and Human Genetics 9 (1), 64-72.

Brook, J. S., Brook, D. W., Gordon, A. S., Whiteman, M. \& Cohen, P. 1990. The psychosocial etiology of adolescent drug use: A family interfactional approach. Genetic, Social and General Psychology Monographs 116 (2), 119-267.

Brown, D. W., Riley, L., Butchart, A., Meddings, D. R., Kann, L. \& Harvey, A. P. 2009. Exposure to physical and sexual violence and adverse health behaviours in African children: Results from the Global School-based Student Health Survey. Bulletin of the World Health Organization 87 (6), 447-455.

Calvert, W. J., Bucholz, K. K. \& Steger-May, K. 2010. Early drinking and its association with adolescents' participation in risky behaviours. Journal of the American Psychiatric Nurses Association 16(4), 239-251.

Carstairs, V. \& Morris, R. 1991. Deprivation and health in Scotland. Aberdeen: Aberdeen University Press.

Cavelaars, A. E., Kunst, A. E., Geurts, J. J., Crialesi, R., Grötvedt, L., Helmert, U., Lahelma, E., Lundberg, O., Matheson, J., Mielck, A., Rasmussen, N. K., Regidor, E., do Rosário-Giraldes, M., Spuhler, T. \& Mackenbach, J. P. 2000. Educational differences in smoking: international comparison. British Medical Journal 320 (7242), 1102-1107.

Chaiton, M. O., Cohen, J. E., O'Loughlin, J. \& Rehm, J. 2009. A systematic review of longitudinal studies on the association between depression and smoking in adolescents. BMC Public Health 9, 356.

Chaloupka, F. J. 2003. Contextual factors and youth tobacco use: policy lingages. Addiction 98 (Supplement 1), 147-149.

Chaloupka, F. J., Grossman, M. \& Saffer, H. 2002. The effect of price on alcohol consumption and alcohol-related problems. Alcohol Research \& Health 26 (1), 22-24.

Chassin, L., Pitts, S. \& Prost, J. 2002. Binge drinking trajectories from adolescence to emerging adulthood in a high-risk sample: predictor of substance abuse outcomes. Journal of Consulting and Clinical Psychology 70 (1), 67-78.

Cheng, J., Yang, X. \& Hu, P. 2003. Youth risk behaviour survey among senior high school students in Beijing. Chinese Journal of School Doctor 17, 203206.

Cho, H. J. \& Khang, Y. H. 2010. Family Affluence Scale, other socioeconomic position indicators, and self-rated health among South Korean adolescents: findings from the Korea Youth Risk Behavior Web-based Survey (KYRBWS). Journal of Public Health 18 (2), 169-178. 
Chou, C.P., Li, Y., Unger, J.B., Xia, J., Sun, P., Guo, Q., Shakib, S., Gong, J., Xie, B., Liu, C. H., Azen, S., Shan, J. G,, Ma, H. Y., Palmer, P., Gallaher, P. \& Johnson, C. A. 2006. A randomized intervention of smoking for adolescents in urban Wuhan, China. Preventive Medicine 42 (4), 280-285.

Choudhury, S., Blakemore, S. J. \& Charman, T. 2006. Social cognitive development during adolescence. Social Cognitive and Affective Neuroscience 1 (3), 165-174.

Chung, T., Smith, G. T., Donovan, J. E., Windle, M., Faden, V. B., Chen, C. M. \& Martin, C. S. 2012. Drinking frequency as a brief screen for adolescent alcohol problems. Pediatrics 129 (2), 205-212.

Clark, D. B., Chung, T. \& Martin, C. 2006. Alcohol use frequency as a screen for alcohol use disorders in adolescents. International Journal of Adolescent Medicine and Health 18 (1), 181-187.

Clark, D. B., Kirisci, L. \& Moss, H. B. 1998. Early adolescent gateway drug use in sons of fathers with substance use disorders. Addictive Behaviours 23, 561-566.

Clark, D. B. \& Kirisci, L. 1996. Posttraumatic stress disorder, depression, alcohol use disorders and quality of life in adolescents. Anxiety 2, 226-233.

Coate, D. \& Grossman, M. 1988. Effects of alcohol beverage prices and legal drinking ages on youth alcohol use. Journal of Law and Economics 31 (1), 145-171.

Cochrane, J., Chen, H., Conigrave, K. M. \& Hao, W. 2003. Alcohol use in China. Alcohol and Alcoholism 38 (6), 537-542.

Cummings, P. \& Quan, L. 1999. Trends in unintentional drowning: the role of alcohol and medical care. The Journal of the American Medical Association 281, 2198-2202.

Currie, C., Elton, R. A., Todd, J. \& Platt, S. 1997. Indicators of socioeconomic status for adolescents: the WHO Health Behaviour in School-aged Children survey. Health Education Research 12 (3), 385-397.

Currie, C., Molcho, M., Boyce, W., Holstein, B. E., Torsheim, T. \& Richter, M. 2008b. Researching health inequalities in adolescents: the development of the Health Behaviour in School-Aged Children (HBSC) Family Affluence Scale. Social Science \& Medicine 66, 1429-1436.

Currie, C., Nic Gabhainn, S., Godeau, E., Roberts, C., Smith, R., Currie, D., Pickett, W., Morgan, A. \& Barnekow, V. (Eds.) 2008a. Inequalities in young people's health: HBSC international report from the 2005/2006 survey. Copenhagen: WHO Regional Office for Europe.

Currie, C., Nic Gabhainn, S., Godeau, E. \& the International HBSC Network Coordinating Committee. 2009. The Health Behaviour in School-aged Children: WHO collaborative cross-national (HBSC) study: origins, concept, history and development 1982-2008. International Journal of Public Health 54 (Supplement 1), 131-139. 
Currie, C., Roberts, C., Morgan, A., Smith, R., Settertobulte, W., Samdal, O. \& Barnekow, V. (Eds.) 2004. Young people's health in context: international report from the HBSC 2001/02 survey. WHO Policy Series: Health policy for children and adolescents. Copenhagen: WHO Regional Office for Europe.

Currie, C., Samdal, O., Boyce, W. \& Smith, R. (Eds.) 2001. Health Behaviour in Schoolaged Children: a WHO cross-national study (HBSC), research protocol for the 2001/2002 Survey. Edinburgh: CAHRU, University of Edinburgh.

Currie, C., Zanotti, C., Morgan, A., Currie, D., de Looze, M., Roberts, C., Samdal, O., Smith O., R. R. \& Barnekow, V. (Eds.) 2012. Social determinants of health and well-being among young people. Health Behaviour in Schoolaged Children (HBSC) study: international report from the 2009/2010 survey. Copenhagen: WHO Regional Office for Europe 2012.

Dalstra, J., Kunst, A. E. \& Mackenbach, J. P. 2006. A comparative appraisal of the relationship of education, income and housing tenure with less than good health among the elderly in Europe. Social Science and Medicine 62, 2046-2060.

Department of Health and Social Security. 1980. Inequalities in health: report of a research working Group. London: Department of Health and Social Security.

Diego, M. A., Field, T. M. \& Sanders, C. E. 2003. Academic performance, popularity and depression predict adolescent substance use. Adolescence 38 (149), 35-42.

DiLiberti, J. H. 2000. The relationship between social stratification and ill-cause mortality among children in the United States. Pediatrics 105 (1), 19681992.

Doku, D., Koivusilta, L., Rainio, S. \& Rimpelä, A. 2010. Socioeconomic differences in smoking among Finnish adolescents from 1977 to 2007. Journal of Adolescent Health 47 (5), 479-487.

Dolcini, M. M., Adler, N. E. \& Ginsberg, D. 1996. Factors influencing agreement between self-reports and biological measures of smoking among adolescents. Journal of Research on Adolescence 6 (4), 515-542.

Donnelly, J., Yong, M., Pearson, R., Penhollow, T. M. \& Hernandez, A. 2008. Area specific self-esteem, values, and adolescent substance use. Journal of Drug Education 38 (4), 389-403.

Donovan, J. E. 2004. Adolescent alcohol initiation: a review of psychosocial risk factors. Journal of Adolescent Health 35 (6), 529. e7-e18.

Du, S., Lu, B., Zhai, F. \& Popkin, B. 2002. A new stage of the nutrition transition in China. Public Health Nutrition 51 (1A), 169 -174. 
Duncan, S. C., Alpert, A., Duncan, T. E. \& Hops, H. 1997. Adolescent alcohol use development and young adult outcomes. Drug Alcohol Depend 49, 39-48.

Duncan, S. C., Duncan, T. E. \& Hops, H. 1998. Progression of alcohol, cigarette, and marijuana use in adolescence. Journal of Behavioural Medicine 21 (4), 375-388.

Durrant, R. \& Thakker, J. 2003. Substance use \& abuse: Cultural and historical perspectives. Thousand Oaks, CA: SAGE.

Eaton, D. K., Kann, L., Kinchen, S., Shanklin, S., Flint, K. H., Hawkins, J., Harris, W. A., Lowry, R., McManus, T., Chyen, D., Whittle, L., Lim, C. \& Wechsler, H. 2012. Youth Risk Behaviour Surveillance-United States 2011. Morbidity and Mortality Weekly Report 61, 4.

Elgar, F. J., Roberts, C., Tudor-Smith, C. \& Moore, L. 2005. Validity of selfreported height and weight and predictors of bias in adolescents. Journal of Adolescent Health 37, 371-375.

Ellickson, P. L., Collins, R. L., Hambarsoomians, K. \& McCaffrey, D. F. 2005. Does alcohol advertising promote adolescent drinking? Results from a longitudinal assessment. Addiction 100 (2), 235-246.

Emery, S., White, M. M. \& Pierce, J.P. 2001. Does cigarette price influence adolescent experimentation? Journal of health Economics 20, 261-270.

Eriksen, M., Mackay, J. \& Ross, H. 2012. The Tobacco Atlas. $4^{\text {th }}$ edition. Atlanta: American Cancer Society.

Farrell, A. D., Danish, S. J. \& Howard, C. W. 1992. Risk factors for drug use in urban adolescents: identification and cross-validation. American Journal of Community Psychology 20, 263-286.

Ferrence, R. 2001. Diffusion theory and drug use. Addition 96, 165-173.

Finkelstein, D. M., Kubzansky, L. D. \& Goodman, E. 2006. Social status, stress, and adolescent smoking. Journal of Adolescent Health 39 (5), 678-685.

Finney, S. J. \& DiStefano, C. 2006. Non-normal and categorical data in structural equation modelling. In G. R. Hancock \& R. O. Mueller (Eds.) Structural equation modelling: A second course. Greenwich: Information Age Publishing.

Finnish Federation of the Brewing and Soft Drinks Industry. 2012. Tax increases lower sales of Finnish beer. Retrieved on 4.3.2013 from http://www.panimoliitto.fi/panimoliitto/en/press_releases/2012/pressrelease21032012.html

Fowler, T., Shelton, K., Lifford, K., Rice, F., McBride, A., Nikolov, I., Neale, M. C., Harold, G., Thapar, A. \& van den Bree, M. B. 2007. Genetic and environmental influences on the relationship between peer alcohol use and own alcohol use in adolescents. Addiction 102, 894-903. 
Galobardes, B., Shaw, M., Lawlor, D. A., Lynch, J. W. \& Smith, G. D. $2006 a$. Indicators of socioeconomic position (part 1). Journal of Epidemiology \& Community Health 60, 7-12.

Galobardes, B., Shaw, M., Lawlor, D. A., Lynch, J. W. \& Smith, G. D. 2006 b. Indicators of socioeconomic position (part 2). Journal of Epidemiology \& Community Health 60, 95-101.

Gecková, A. 2002. Inequality in health among Slovak adolescents. Doctoral thesis. Groningen: Northern Centre for Healthcare Research.

Gecková, A, Stewart, R., van Dijk, J. P., Orosová, O., Groothoff, J. W. \& Post D. 2005. Influence of socio-economic status, parents and peers on smoking behaviour of adolescents. European Addiction Research 11, 204-209.

General Sport Administration of China. 2006. Report on the Second National Physical Fitness Surveillance. Beijing: People's Sport Publishing House.

General Sport Administration of China. 2011. Report on the Third National Physical Fitness Surveillance. Beijing: People's Sport Publishing House.

Gilman, S. E., Rende, R., Boergers, J., Abrams, D. B., Buka, S. L., Clark, M. A., Colby, S. M., Hitsman, B., Kazura, A. N., Lipsitt, L. P., Lloyd-Richardson, E. E., Rogers, M. L., Stanton, C. A., Stroud, L. R. \& Niaura, R. S. 2009. Parental smoking and adolescent smoking initiation: an intergenerational perspective on tobacco control. Pediatrics 123 (2), e274-e281.

Giskes, K., Kunst, A. E., Benach, J., Borrell, C., Costa, G., Dahl, E., Dalstra, J. A., Judge, K., Lahelma, E., Moussa, K., Ostergren, P. O., Platt, S., Prattala, R., Rasmussen, N. K. \& Mackenbach, J. P. 2005. Trends in smoking behaviour between 1985 and 2000 in nine European countries by education. Journal of Epidemiology and Community Health 59 (5), 395-401.

Godeau, E. 2000. Jeux symboliques autour de l'alcool et de l'ivresse: l'exemple du baptême des internes en medicine [Symbolic games around alcohol and drunkenness]. In F. Navarro, E. Godeau \& C. Vialas (Eds.) Actes du colloque les jeunes et l'alcool en Europe [Proceedings of the conference on alcohol use of young people in Europe]. Toulouse: Editions Universitaires du Sud.

Gonçalves, A., Matos, M. G., Marques, F. V., Gaspar, T., Faustino, M., Rosabal, M., Moniz, J. C. \& Torgal, J. 2005. A saúde e estilo de vida dos adolescentes Cabo-verdianos frequentando e ensino secundário [The Health and lifestyle of Cabo Verde adolescents]. Lisbon: Istitute of Hygience \& Tropical Medicine, New University of Lisbon.

Griebler, R., Molcho, M., Samdal, O., Inchley, J. Dür, W. \& Currie, C. (Eds.) 2010. Health Behaviour in School-aged Children: a World Health Organization cross-national study. Research protocol for the for the 2009/2010 survey. Vienna: LBIHPR \& Edinburgh: CAHRU. 
Gu, D., Kelly, T. N.,Wu, X., Chen, J., Samet, J. M., Huang, J. F., Zhu, M., Chen, J. C., Chen, C. S., Duan, X., Klag, M. J. \& He, J. 2009. Mortality attributable to smoking in China. The New England Journal of Medicine 360, 150-159.

Hanson, M. D. \& Chen, E. 2007. Socioeconomic status and health behaviours in adolescence: a review of the literature. Journal of Behavioural Medicine 30 (3), 263-285.

Hantrais, L. \& Mangen, S. (Eds.) 1996. Cross-national research methods. London and New York: Pinter.

Hao, W., Chen, H. \& Su, Z. 2005. China: alcohol today. Addiction 100, 737-741.

Hardy, L. L., Booth, M. L. \& Okely, A. D. 2007. The realiability of the adolescent sedentary activity questionnaire (ASAQ). Preventive Medicince 45 (1), 7174 .

Harkness, J. 2003. Questionnaire Translation. In J. F. Harkness, J., F. J. R. van de Vijver \& P. P. Mohler (Eds.) Cross-cultural survey methods. Hoboken: John Wiley \& Sons, 35-56.

Harrison, L. \& Gardiner, E. 1999. Do the rich really die young? Alcohol-related mortality and social class in Great Britain,1988-94. Addiction 94, 1871-1880.

Haugland, S. \& Wold, B. 2001. Subjective health complaints in adolescenceReliability and validity of survey methods. Journal of Adolescence 24, 611-624.

Hawkins, J. D., Catalano, R. F. \& Miller, J. Y. 1992. Risk and protective factors for alcohol and other drug problems in adolescence and early adulthood: implications for substance use prevention. Psychological Bulletin 112, 64105.

Heikkinen, A. M., Broms, U., Pitkäniemi, J., Koskenvuo, M. \& Meurman, J. 2009. Key factors in smoking cessation intervention among 15-16-year-olds. Behavioural Medicine 35 (3), 93-99.

Hennekens, C. H., Willett, W., Rosner, B., Cole, D. S. \& Mayrent, S. L. 1979. Effects of beer, wine and liquor in coronary deaths. The Journal of the American Medical Association 242 (18), 1973-1974.

Henriksen, L. \& Jackson, C. 1999. Reliability of children's self-reported cigarette smoking. Addictive Behaviours 24 (2), 271-277.

Hibell, B., Andersson, B., Ahlström, S., Balakireva, O., Bjarnason, T., Kokkevi, A. \& Morgan, M. 2000. The 1999 ESPAD report: substance use among students in 35 European countries. Stockholm: The Swedish Council for Information on Alcohol and Other Drugs.

Hibell, B., Guttormsson, U., Ahlström, S., Balakireva, O., Bjarnason, T., Kokkevi, A. \& Kraus, L. 2009. The 2007 ESPAD report: substance use among students in 35 European countries. Stockholm: The Swedish Council for Information on Alcohol and Other Drugs.

Hibell, B., Guttormsson, U., Ahlström, S., Balakireva, O., Bjarnason, T., Kokkevi, A. \& Kraus, L. 2012. The 2011 ESPAD Report: substance use among 
students in 36 European countries. Stockholm: The Swedish Council for Information on Alcohol and Other Drugs.

Hill, S. Y. \& Steinhauer, S. R. 1993. Assessment of prepubertal and postpubertal boys and girls at risk for developing alcoholism with P300 froma visual discrimination task. Journal of Studies on Alcohol 54, 350-358.

Hill, K. G., White, H. R., Chung, I. J., Hawkings, J. D. \& Catalano, R. F. 2000. Early adult outcomes of adolescent binge drinking: person- and variablecentered analyses of binge drinking trajectories. Alcoholism, Clinical and Experimental Research 24 (6), 892-901.

Jackson, C. \& Dickinson, D. 2006. Enabling parents who smoke to prevent their children from initiating smoking: results from a 3-year intervention evaluation. Archives of Pediatrics \& Adolescent Medicine 160 (1), 56-62.

Jensen, E. J. \& Overgaard, E. 1993. Investigation of smoking habits among 1417-year-old boarding school pupils: factors which influence smoking status. Public Health 107, 117-123.

Jha, P., Peto, R., Zatonski, W., Boreham, J., Jarvis, M. J. \& Lopez, A. D. 2006. Social inequalities in male mortality and in male mortality from smoking: Indirect estimation from national death rates in England and Wales, Poland and North America. Lancet 368, 367-370.

Ji, C. (Ed.) 2007. Report of the China Adolescent Behavioural Risk Survey 2005. Beijing: Peking Univeristy Medical Press.

Jimenez, T. I., Musitu, G. \& Murgui, S. 2008. Family functioning and substance use in adolescents: the mediator role of self-esteem. International Journal of Clinical and Health Psychology 8 (1), 139-151.

Johnston, L. D., O'Malley, P. M., Bachman, J. G. \& Schulenberg, J. E. 2011 Monitoring the future national results on adolescent drug use: overview of key findings. Bethesda: National Institute on Drug Abuse.

Jones, S. C. \& Magee, C. A. 2011. Exposure to alcohol advertising and alcohol consumption among Australian adolescents. Alcohol and Alcoholism 46 (5), 630-673.

Karvonen, S. \& Rahkonen, O. 2011. Subjective social status and health in young people. Sociology of Health \& Illness 33 (3), 372-383.

Karvonen, S. \& Rimpelä, A. 1996. Socio-regional context as a determinant of adolescents' health behaviour in Finland. Social Science \& Medicine 43 (10), 1467-1474.

Karvonen, S., Abel, T., Calmonte, R. \& Rimpelä A. 2000. Patterns of healthrelated behaviour and their cross-cultural validity - A comparative study on two populations of young people. Sozial-und Präventivmedizin 45, 3545.

Karvonen, S., West, P., Sweeting, H., Rahkonen, O. \& Young, R. 2001. Lifestyle, social class and health-related behaviour: A cross-cultural comparison of 
15 year olds in Glasgow and Helsinki. Journal of Youth Studies 4 (4), 393413.

Kawachi, I., Subramanian, S. V. \& Almeida-Filho, N. 2002. A glossary for health inequalities. Journal of Epidemiology \& Community Health 56, 647-652.

King, A., Wold, B., Tudor-Smith, C. \& Harel, Y. 1996. The health of youth: a cross-national survey. WHO Regional Publications, European Series No. 69.

Kinnunen, J. M., Lindfors, P., Pere, L., Ollila, H., Samposalo, H. \& Rimpelä, A. 2013. Nuorten terveystapatutkimus 2013: Nuorten tupakkatuotteiden ja päihteiden käyttö 1977-2013. [The Adolescent Health and Lifestyle Survey 2013. Adolescent smoking, alcohol and substance use in 1977-2013]. Sosiaali- ja terveysministeriön raportteja ja muistiota, 16. Helsinki: Sosiaali- ja Terveysministeriö.

Klingemann, H. \& Gmel, G. Introduction: Social consequences of alcohol - the forgotten dimension? In H. Klingemann \& G. Gmel (Eds.) Mapping the social consequences of alcohol consumption. Dordrecht: Kluwer, 1-9.

Kohl, H. W., Fulton, J. E. \& Casperson, C. J. 2000. Assessment of physical activity among children and adolescents: a review and synthesis. Preventive Medicine 31 (Supplement), S54-S76.

Koivusilta, L. 2000. Health-related selection into educational tracks. A mechanism producing socio-economic health differences. Doctoral thesis. Turku: Department of Public Health, Univeristy of Turku.

Kouvonen, A. \& Lintonen, T. 2002. Adolescent part-time work and heavy drinking in Finland. Addiction 97, 311-318.

Krahn, D., Piper, D., King, M., Olson, L., Kurth, C. \& Moberg, D. P. 1996. Dieting in sixth grade predicts alcohol use in ninth grade. Journal of Substance Abuse 8 (3), 293-301.

Kraus, L., Metzner, C. \& Piontek, D. 2010. Alcopops, alcohol consumption and alcohol relatedproblems in a sample of German adolescent: is there an alcopop-specific effect? Drug and Alcohol Dependence. 110(1-2), 15-20.

Kreiner, S. 2009. A guided tour through DIGRAM 2.0: Analysis of contingency tables by chain graph models. Copenhagen: Department of Biostatistics, University of Copenhagen.

Kreiner, S. \& Christensen, K. B. 2011. Item screening in Graphical Loglinenear Rasch Models. Psychometrika 76 (2), 228-256.

Kunst, A. E., Cavelaars, A., Groenhof, F., Geurts, J., Mackenbach, J. P. \& EU Working Group on Socioeconomic Inequalities in Health. 1996. Socioeconomic inequalities in morbidity and mortality in Europe: a comparative study. Volume I: Main Report. Rotterdam: Erasmus University Rotterdam 35, 87-89.

Kunst, A. E., Feikje, G. \& Mackenbac, J. P. 1998. Occupational class and cause specific mortality in middle aged men in 11 European countries: 
comparison of population cased studies. British Medical Journal 316, 1636-1642.

Kuntsche, E., Rehm, J. \& Gmel G. 2004. Characteristics of binge drinkers in Europe. Social Science \& Medicine 59, 113-127.

Kuntsche, E., Rossow, I., Simons-Morton, B., Ter Bogt, T., Kokkevi, A. \& Godeau, E. 2013. Not early drinking but early drunkenness is a risk factor for problem behaviours among adolescents from 38 European and North American countries. Alcoholism: Clinical and Experimental Research 37 (2), 308-314.

La Greca, A. M., Prinstein, M. J. \& Fetter, M. D. 2001. Adolescent peer crowd affiliation: linkages with health-risk behaviours and close friendships. Journal of Pediatric Psychology 26, 131-143.

Laaksonen, M., Talala, K., Martelin, T., Rahkonen, O., Roos, E., Helakorpi, S., Laatikainen, T. \& Prattala, R. 2008. Health behaviours as explanations for educational level differences in cardiovascular and all-cause mortality: a follow-up of 60000 men and women over 23 years. European Journal of Public Health 18 (1), 38-43.

Lance, P. M., Akin, J. S., Dow, W. H. \& Loh, C. P. 2004. Is cigarette smoking in poorer nations highly sensitive to price? Evidence from Russia and China. Journal of Health Economics 23 (1), 173-189.

Lancet. 2008. Calling time on young people's alcohol consumption. The Lancet 371 (9616), 871.

Landis, J. R. \& Koch, G. G. 1977. The measurement of observer agreement for categorical data. Biometrics 33, 159-174.

Lando, H. A., Hipple, B. J., Muramoto, M., Klein, J. D., Prokhorov, A. V., Ossip, D. J. \& Winickoff, J. P. 2010. Tobacco is a global paediatric concern. Bulletin of the World Health Organization 88, 2-2.

Le Deu, F., Parekh, R., Zhang, F. \& Zhou, G. 2012. Healthcare in China: entering uncharted waters. China: McKinsey \& Company. Retrieved on 28.2.2013 from http://www.mckinseychina.com/wp-content/uploads/2012/08/ healthcare- in-china-entering-uncharted-waters.pdf

Leffingwell, T. R., Cooney, N. J., Murphy, J. G., Luczak, S., Rosen, G., Dougherty, D. M. \& Barnett, N. P. 2013. Continuous objective monitoring of alcohol use: twenty-first century measurement using transdermal sensors. Alcoholism: Clinical and Experimental Research 37 (1), 16-22.

Lerner, R. M. \& Steinberg, L. D. 2004. Handbook of Adolescent Psychology. 2nd Edition. Hoboken, NJ: John Wiley \& Sons.

Lerman, C. E., Schnoll, R. A. \& Munafo, M. R. 2007. Genetics and smoking cessation: improving outcomes in smokers at risk. American Journal of Preventive Medicine 33 (6), S398-S405.

Li, L., Yong, H. H., Fong, G. T., Thompson, M. E., Jiang, Y., Yang, Y., Sirirassamee, B., Hastings, G. \& Harris, F. 2009. Reported awareness of 
tobacco advertising and promotion in China compared to Thailand, Australia and the USA. Tobacco Control 18 (3), 222-227.

Li, M. D., Cheng, R., Ma, J. Z. \& Swan, G. E. 2003. A meta-analysis of estimated genetic and environmental effects on smoking behaviour in male and female adult twins. Addiction 98, 23-31.

Lin, Y. C. 2011. Assessing the use of the Family Affluence Scale as socioeconomic indicators for researching health inequalities in Taiwan adolescents. Social Indicators Research 102, 463-475.

Lintonen, T. 2001. Drinking patterns among Finnish fourteen year-olds from 1977 to 1999. Doctoral thesis. Acta Universitatis Tamperensis 832. Tampere: University of Tampere.

Lintonen, T., Ahlström, S. \& Metso, L. 2004. The reliability of self-reported drinking in adolescence. Alcohol and Alcoholism 39 (4), 362-368.

Lintonen, T., Karlsson, T., Nevalainen, J. \& Konu, A. 2013. Alcohol policy changes and trends in adolescent drinking in Finland from 1981 to 2011. Alcohol and Alcoholism 48(5), 620-626.

Lintonen, T., Rimpelä, M., Vikat, A. \& Rimpelä, A. 2000. The effect of societal changes on drunkenness trends in early adolescence. Health Education Research 15, 261-269.

Lintonen, T., Wilska, T., Koivusilta, K. \& Konu, A. 2007. Trends in disposable income among teenage boys and girls in Finland from 1977 to 2003. International Journal of Consumer Studies 31, 340-348.

Lisle, E. 1985. Validation in the social science by international comparison. Cross-National Research Papers 1 (1), 11-28.

Liu, X., Liu, L., Owens, J. A. \& Kaplan, D. L 2005. Sleep patterns and sleep problems among schoolchildren in the United States and China. Pediatrics 115 (1), 241-249.

Liu, Y., Wang, M., Tynjälä, J., Lv, Y., Villberg, J., Zhang, Z. \& Kannas, L. 2010. Test-retest reliability of selected items of Health Behaviour in School-aged Children (HBSC) survey questionnaire in Beijing, China. BMC Medical Research Methodology 10, 73.

Liu, Y., Wang, M., Villberg, J., Torsheim, T., Tynjälä, J., Lv, Y. \& Kannas, L. 2012. Reliability and validity of family affluence scale (FAS II) among adolescents in Beijing, China. Child Indicators Research 5 (2), 235-251.

Liu, Y., Wang, M., Tynjälä, J., Villberg, J., Lv, Y. \& Kannas, L. 2013. Socioeconomic inequalities in alcohol use of adolescents: the differences between China and Finland. International Journal of Public Health 58 (2), 177-185.

Liu, Z., Zhou, W., Lian, Z., Mu, Y., Cai, Z. \& Cao, J. 2001. The use of psychoactive substances among adolescent students in an area in the south-west of China. Addition 96, 247-250. 
Lopez, A. D., Collishaw, N. E. \& Piha, T. 1994. A descriptive model of the cigarette epidemic in developed countries. Tobacco Control 3, 242-247.

Lovato, C., Watts, A. \& Stead, L. F. 2011. Impact of tobacco advertising and promotion on increasing adolescent smoking behaviours. Cochrane Database of Systematic Reviews 10, CD003439.

Loveland-Cherry, C. J., Ross, L. T. \& Kaufman, S. R. 1999. Effects of a homebased family intervention on adolescent alcohol use and misuse. Journal of Studies on Alcohol Supplement 13, 94-102.

Luepker, R. V., Pechacek, T. F., Murray, D. M., Anderson Johnson, C., Hund, F. \& Jacobs, D. R. 1981. Saliva thiocyanate: a chemical indicator of cigarette smoking in adolescents. American Journal of Public Health 71, 1320-1324.

Luo, C., Peng, N. \& Zhu, W. 2003. Risk behaviours of adolescents in Shanghai: smoking, drinking and addictive drug use. Chinese Journal of School Doctor 17, 104-107.

Macintyre, S. 1986. The patterning of health by social position in contemporary Britain: directions for sociological research. Social Science and Medicine 23 (4), 393-415.

Macintyre, S. 1987. West of Scotland Twenty-07: Health in the Community. The survey's background and rational. Working Paper No. 7. Glasgow: MRC.

Macintyre, S. \& Hunt, K. 1997. Socio-economic position, gender and health. How do they interact? Journal of Health Psychology 2 (3), 315-334.

Mackenbach, J. P., Stirbu, I., Roskam, A. J. R., Schaap, M. M., Menvielle, G., Leinsalu, M. \& Kunst, A. E. 2008. Socioeconomic inequalities in health in 22 European countries. New England Journal of Medicine 358, 2468-2481.

Mackenbach, J. P., van de Mheen, H. \& Stronks, K. 1994. A prospective cohort study investigating the explanation of socio-economic inequalities in health in The Netherlands. Social Science and Medicine 8, 299-308.

Maes, L. A., Vereecken, C., Vanobbergen, J. \& Honkala, S. 2006. Tooth brushing and social characteristics of families in 32 countries. International Dental Journal 56, 159-167.

Magklara, K., Skapinakis, P., Niakas, D., Bellos, S., Zissi, A., Stylianidis, S. \& Mavreas, V. 2010. Socioeconomic inequalities in general and psychological health among adolescents: a cross-sectional study in senior high schools in Greece. International Journal for Equity in Health 9, 3.

Mahler, H. 1981. The meaning of Health For All by the year 2000. World Health Forum, 2 (1), 5-22.

Marmot, M. 2005. Social determinants of health inequalities. Lancet 365, 10991104.

Marshal, W. 1978. Puberty. In F. Falkner \& J. Tanner (Eds.) Human growth, Vol. 2. New York: Plenum.

Marsiglia, F. F., Ayers, S., Gance-Cleveland, B., Mettler, K. \& Booth, J. 2012. Beyond primary prevention of alcohol use: a culturally specific secondary 
prevention program for Mexican heritage adolescents. Prevention Science 13 (3), 241-251

Martin, A. 2012. The rise of the alcopop and children drinking. Retrieved on 1.3.2013 from http://www.alcoholissues.co.uk/rise-alcopop-childrendrinking.html

McGue, M., Elkins, I. \& Iacono, W. G. 2000. Genetic and environmental influences on adolescent substance use and abuse. American Journal of Medical Genetics 96, 671-677.

McGue, M., Iacono, W. G., Legrand L. N., Malone, S. \& Elkins, I. 2001. Origins and consequences of age at first drink. I. Associations with substance-use disorders, disinhibitory behaviour and psychopathology, and P3 amplitude. Alcoholism: Clinical Experimental Research 25, 1156-1165.

McQueen, D. V. 2009. 25 years of HBSC: challenges and success. International Journal of Public Health 54, S123-S124.

Mehrens, W. A. \& Lehman, I. J. 1991. Measurement and Evaluation in Education and Psychology. $4^{\text {th }}$ edition. Orlando, Fl: Holt, Rinehart and Winston.

Melotti, R., Heron, J., Hickman, M., Macleod, J., Araya, R. \& Lewis, G. 2011. Adolescent alcohol and tobacco use and early socioeconomic position: the ALSPAS birth cohort. Pediatrics 127 (4), e948-955.

Metso, L., Ahlström, S., Huhtanen P., Leppänen, M. \& Pietilä, E. 2009. Alcohol and drug use among adolescents in Finland 1995-2007. ESPAD survey results. Helsinki: The National Institute for Health and Welfare (THL), Report 6/2009.

Mikkonen, P., Leino-Arjas, P., Remes, J., Zitting, P., Taimela, S. \& Karppinen, J. 2008. Is smoking a risk factor for low back pain in adolescents? A prospective cohort study. Spine 33 (5), 527-532.

Millett, C., Lee, J. T., Gibbons, D. C. \& Glantz, S. A. 2011. Increasing the age for the legal purchase of tobacco in England: impacts on socio-economic disparities in youth smoking. Thorax 66 (10), 862-865.

Ministry of Commerce of China. 2005. Regulations on alcoholic beverages trade. No. 25. Retrieved on 4.3.2013 from http://www.gov.cn/flfg/200511/10/content_94951.htm

Ministry of Social Affairs and Health of Finland. 1997. The Alcohol Act. No. 1143/1994. Retrieved on 3.3.2013 from http://www.finlex.fi/fi/laki/kaannokset/1994/en19941143.pdf

Ministry of Social Affairs and Health of Finland. 2010. Tobacco Act. No. 693/1976. Retrieved on 4.3.2013 from http://savutonsuomifi. virtualserver21.nebula.fi/doc/ENGLISH-Translation-2010.pdf

Mistry, R., McCarthy, W., de Vogli, R., Crespi, C. M., Wu, Q. \& Patel M. 2011. Adolescent smoking risk increases with wider income gaps between rich and poor. Health \& Place 17, 222-229. 
Moffitt, T. E. 2006. A review of research on the taxonomy of life-course persistent versus adolescence-limited antisocial behaviour. In F. T. Cullen, J. P. Wright \& K. R. Blevins (Eds.) Taking stock: the status of criminological theory. New Brunswick, New Jersey: Transaction Publishers.

Molcho, M., Nic Gabhainn, S. \& Kelleher, C. 2007. Assessing the use of the Family Affluence Scale (FAS) among Irish schoolchildren. Irish Medical Journal 100 (8), 37-39.

Morgenstern, M., Poelen, E. A., Scholte, R., Karlsdottir, S., Jonsson, S. H., Mathis, F., Faggiano, F., Florek, E., Sweeting, H., Hunt, K., Sargent, J. D. \& Hanewinkel, R. 2011. Smoking in movies and adolescent smoking: crosscultural study in six European countries. Thorax 66 (10), 875-883.

Muller, S., Piontek, D., Pabst, A., Baumeister, S. E. \& Kraus L. 2010. Changes in alcohol consumption and beverage preference among adolescents after the introduction of the alcopops tax in Germany. Addiction 105 (7), 1205-1213.

Munro, G. \& Learmonth, A. 2004. 'An unacceptable risk': the problem of alcoholic milk. Drug and Alcohol Review 23 (3), 345-349.

Murphey, D., Vaughn, B., Barry, M. \& Terzian, M. 2012. Alcohol use. Washington DC: Child Trends, 34.

Murray, C. J. L., Michaud, C. M., McKenna, M. T. \& Marks, J. S. 1998. US patterns of mortality by county and race: 1965-94. Cambridge: Harvard Centre for Population and Development Studies.

Muthén, L. K. \& Muthén, B. O. 2010. Mplus User's Guide. $6^{\text {th }}$ edition. Los Angeles: Muthén \& Muthén.

Mäkelä, P. \& Österberg, E. 2009. Weakening of one more alcohol control pillar: a review of the effects of the alcohol tax cuts in Finland in 2004. Addiction 104 (4), 554-563.

Mäkelä, P., Keskimäki, I. \& Koskinen, S. 2003. What underlies the high alcohol related mortality of the disadvantaged: high morbidity or poor survival? Journal of Epidemiology and Community Health 57, 981-986.

National Bureau of Statistics of China. 2013. China Statistical Yearbook 2012. Beijing: China Statistics Press.

National Centre for Health Statistics. 2008. Health United States 2008: with chart book on trends in the Health of Americans. Hyattsville, MD.

National Highway Traffic Safety Administration. 2000. Traffic safety facts 2000: Young drivers. Washington, DC: U.S. Department of Transportation.

Newman, I. 2002. Cultural aspects of drink alcohol controls in China. Global Alcohol Policy Alliance 1, 18-21.

O'Loughlin, J. O., DiFranza, J., Tyndale, R. F., Meshefedjian, G., McMillanDavey, E., Clarke, P. B., Hanley, J. \& Paradis, G. 2003. Nicotinedependence symptoms are associated with smoking frequency in adolescents. American Journal of Preventive Med 25 (3), 219-225. 
O’Loughlin, J. O., Karp, I., Koulis, T., Paradis, G. \& DiFranza, J. 2009. Determinants of first puff and daily cigarette smoking in adolescents. American Journal of Epidemiology 170 (5), 585-597.

Oakes, J. M. \& Rossi, P. H. 2003. The measurement of SES in health research: current practice and steps toward a new approach. Social Science \& Medicine 56 (4), 769-784.

Ojala, K., Vuori, M., Välimaa, R., Villberg, J., Tynjälä, J. \& Kannas, L. 2005. Reasons for exercise inventory koulukyselyssä: mittarin reliabiliteetti-ja rakennevaliditeettitarkastelua. [Reasons for exercise inventory in a school survey: contemplations of the inventory's reliability and structure validity]. Liikunta \& Tieta 42 (6), 30-38.

Ostrove, J. M. \& Adler, N. E. 1998. Socioeconomic status and health. Current Opinion in Psychiatry 11, 649-653.

Otsuki, T. A. 2003. Substance use, self-esteem, and depression among Asian American adolescents. Journal of Drug Education 33 (4), 369-390.

Otter, M. E., Mellenbergh, G. J. \& Glopper, K. D. 1995. The relation between informationprocessing variables and test-retest stability for questionnaire items. Journal of Educational Measurement 32 (2), 199-216.

Paavola, M., Vartiainen, E. \& Haukkala, A. 2004. Smoking from adolescence to adulthood: the effects of parental and own socioeconomic status. European Journal of Public Health 14, 417-421.

Pagan, J. L., Rose, R. J., Viken, R. J., Pulkkinen, L., Kaprio, J. \& Dick, D. M. 2006. Genetic and environmental influences on stages of alcohol use across adolescence and into young adulthood. Behaviour Genetics 36, 483-497.

Page, R. M., Dennis, M., Lindsay, G. B. \& Merrill, R. M. 2011. Psychosocial distress and substance use among adolescents in four countries: Philippines, China, Chile, and Namibia. Youth \& Society 43 (3),900-930.

Patrick, D. L., Cheadle, A., Thompson, D. C., Diehr, P., Koepsell, T. \& Kinne, S. 1994. The validity of self-reported smoking: a review and meta-analysis. American Journal of Public Health 84 (7)m 1086-1093.

Patton, G. C., Hibbert, M., Rosier, M. J, Carlin, J. B., Caust, J. \& Bowes, G. 1996. Is smoking associated with depression and anxiety in teenagers? American Journal of Public Health 86 (2), 225-230.

Peng, N., Luo, C. \& Zhu, W. 2003. Risk behaviors of adolescents in Shanghai: knowledge, attitudes and behaviors regarding HIV/AIDS and sexual behaviours. Chinese Journal of School Doctor 17, 97-99.

Pennanen, M. 2012. School achievement, family factors and smoking prevention. Helsinki: The National Institute for Health and Welfare (THL), Research 78/2012.

Perkins, H. W. 2002. Surveying the damage: a review of research on consequences of alcohol misuse in college populations. Journal of Studies on Alcohol and Drugs 14 (supplement), 91-100. 
Peterson, P. L., Hawkins, J. D., Abbott, R. D. \& Catalano, R. F. 1994. Disentangling the effects of parental drinking, family management, and parental alcohol norms on current drinking by black and white adolescents. Journal of Research on Adolescence 4, 203-227.

Phongsavan, P., Olatunbosun-Alakija, A., Havea, D., Bauman, A., Smith, B. J., Galea, G. \& Chen, J. 2005. Health behaviour and lifestyle of Pacific youth surveys: a resource for capacity building. Health Promotion International 20 (3), 238-248.

Pickett, W., Molcho, M., Simpson, K., Janssen, I., Kuntsche, E., Mazur, J., Harel, Y. \& Boyce, W. 2005. Cross-national study of injury and social determinants in adolescents. Injury Prevention 11, 213-218.

Pietilä, A. M., Hentinen, M. \& Myhrman, A. 1995. The health behaviour of northern Finnish men in adolescence and adulthood. International Journal of Nursing Studies 32 (3), 325-338.

Pirkola, S. P., Marttunen, M. J., Henriksson, M. M. \& Isometsa, E. T. 1999. Alcohol-related problems among adolescent suicides in Finland. Alcohol and Alcoholism 34 (3), 320-329.

Plant, M. \& Miller, P. 2001. Young people and alcohol: an international insight. Alcohol and Alcoholism 36 (6), 513-515.

Raisamo, S., Pere, L., Lindfors, P., Tiirikainen, M. \& Rimpelä, A. 2011. Nuorten terveystapatutkimus 2011: Nuorten tupakkatuotteiden ja päihteiden käyttö 1977-2011. [The Adolescent Health and Lifestyle Survey 2011: Adolescent smoking, alcohol and substance use in 1977-2011]. Sosiaali- ja terveysministeriön raportteja ja muistiota, 10. Helsinki: Sosiaali- ja Terveysministeriö.

Randall, V. R. 1999. History of tobacco. Retrieved on 6.5.2013 from http://academic.udayton.edu/health/syllabi/tobacco/history.htm

Rangul, V., Holmen, T. L., Kurtze, N., Cuypers, K. \& Midthjell, K. 2008. Reliability and validity of two frequently used self-administered physical activity questionnaires in adolescents. BMC Medical Research methodology 8, 47.

Rasmussen, M., Due, P., Damsgaard, M. T. \& Holstein, B. 2009. Social inequality in adolescent daily smoking: has it changed over time. Scandinavian Journal of Public Health 37 (3), 287-294.

Ravens-Sieberer, U., Erhart, M., Torsheim, T., Hetland, J., Freeman, J., Danielson, M., Thomas, C. \& the HBSC Positive Health Group. 2008. An international scoring system for self-reported health complaints in adolescents. European Journal of Public Health 18, 294-299.

Rehm, J., Gmel, G., Room, R. \& Ulrich, F. 2001. Average volume of alcohol consumption, drinking patterns and related burden of mortality in young people in established market economies of Europe. European Addiction Research 7, 148-151. 
Rhee, S. H., Hewitt, J. K., Young, S. E., Corley, R. P., Crowley, T. J. \& Stallings, M. C. 2003. Genetic and environmental influences on substance initiation, use, and problem use in adolescents. Archives of General Psychiatry 60, 1256-1264.

Riala, K., Alaraisanen, A., Taanila, A., Hakko, H., Timonen, M. \& Rasanen, P. 2007. Regular daily smoking among 14-year-old adolescents increases the subsequent risk for suicide: The Northern Finland 1966 Birth Cohort Study. Journal of Clinical Psychiatry 68 (5), 775-780.

Richter, L. \& Johnson, P. B. 2001. Current methods of assessing substance use: A review of strengths, problems, and developments. Journal of Drug Issues 31 (4), 809-832.

Richter, M., Erhart, M., Vereecken, C. A., Zambon, A., Boyce, W. \& Nic Gabhainn, S. 2009. The role of behavioural factors in explaining socioeconomic differences in adolescent health: A multilevel study in 33 countries. Social Science \& Medicine 69 (3), 396-403.

Richter, M., Leppin, A. \& Nic Gabhainn, S. 2006. The relationship between parental socio-economic status and episodes of drunkenness among adolescents: findings from a cross-national survey. BMC Public Health 6, 289.

Richter, M. \& Leppin, A. 2007. Trends in socio-economic differences in tobacco smoking among German schoolchildren, 1994-2002. European Journal of Public Health 17 (6), 565-571.

Ritterman, M. L., Fernald, L. C., Ozer, E. J., Adler, N. E., Gutierrez, J. P. \& Syme, S. L. 2009. Objective and subjective social class gradients for substance use among Mexican adolescents. Social Science \& Medicine 68, 1843-1851.

Roberts, C., Freeman, J., Samdal, O., Schnohr, C.W., de Looze, M.E., Nic Gabhainn, S., Iannotti, R., Rasmussen, M. \& the International HBSC Study Group. 2009. The Health Behaviour in School-aged Children (HBSC) study: methodological developments and current tensions. International Journal of Public Health 54(S1), 140-150.

Roberts, C., Currie, C., Samdal, O., Currie, D., Smith, R. \& Maes, L. A. 2007. Measuring the health and health behaviours of adolescents through crossnational survey research: recent developments in the Health Behaviour in School-aged Children (HBSC) study. Journal of Public Health 15, 179-186.

Robinson, T. N., Chen, H. L. \& Killen, J. D. 1998. Television and video exposure and risk of adolescent alcohol use. Pediatrics 105 (5), e54.

Rose, R. J., Dick, D. M., Viken, R. J. \& Kaprio, J. 2001. Gene-environment interaction in patterns of adolescent drinking: regional residency moderates longitudinal influences on alcohol use. Alcoholism: Clinical and Experimental Research 25(5), 637-643.

Rosen, M. 1987. Epidemiology in planning for health. Umeå: Department of Social Medicine. 
Rosenbaum, P. R. 1989. Criterion-related construct validity. Psychometrika 54, 625-633.

Ryan, S. M., Jorm, A. F. \& Lubman, D. I. 2010. Parenting factors associated with reduced adolescent alcohol use: a systematic review of longitudinal studies. Australian and New Zealand Journal of Psychiatry 44, 774-783.

Saffer, H. \& Dave, D. 2006. Alcohol advertising and alcohol consumption by adolescents. Health Economics 15 (6), 617-637.

Sarkola, T., Gissler, M., Kahila, H., Autti-Ramo, I. \& Halmesmaki, E. 2011. Early healthcare utilization and welfare interventions among children of mothers with alcohol and substance abuse: a retrospective cohort study. Acta Paediatrica 100 (10), 1379-1385.

Sawyer, S. M., Afifi, R. A., Bearinger, L. H., Blakemore, S. J., Dick, B., Ezeh, A. C. \& Patton. G. C. 2012. Adolescence: a foundation for future health. Lancet 379 (9826), 1630-1640.

Schilling, E. A., Aseltine, R. H., Glanovsky, J. L., James, A. \& Jacobs, D. 2009. Adolescent alcohol use, suicidal ideation, and suicide attemps. Journal of Adolescent Health 44 (4), 335-341.

Schnohr, C. W., Kreiner, S., Due, P., Currie, C., Boyce, W. \& Diderichsen, F. 2008. Differential item functioning of a family affluence scale: validation study on data from HBSC 2001/02. Social Indicators Research, 89 (1), 79-95.

Schnohr, C. W., Kreiner, S., Rasmussen, M., Due, P. \& Diderichsen, F. 2009. School-related mediators in social inequalities in smoking: a comparative cross-sectional study of 20399 adolescents. International Journal for Equity in Health, 8, 17.

Schulte, M. T., Ramo, D. \& Brown, S. A. 2009. Gender differences in factors influencing alcohol use and drinking progression among adolescents. Clinical Psychology Review 29, 535-547.

Science Daily. 2013. Science reference: drunkenness. Retrieved on 1.3.2013 from http://www.sciencedaily.com/articles/d/drunkenness.htm

Shavers, V. L. 2007. Measurement of socioeconomic status in health disparities research. Journal of the National Medical Association 99 (9), 1013-1023.

Shrewsbury, V. \& Wardle J. 2008. Socioeconomic status and adiposity in childhood: a systematic review of cross-sectional studies 1990-2005. Obesity 16, 275-284.

Shrout, P. E. \& Fleiss, J. L. 1979. Intraclass Correlation: using in assessing rater reliability. Psychological Bulletin 86 (2), 420-428.

Siahpush, M., Borland, R., Taylor, J., Singh, G. K., Ansari, Z. \& Serraglio, A. 2006. The association of smoking with perception of income inequality, relative material well-being, and social capital. Social Science \& Medicine 63, 2801-2812.

Simetin, I. P., Kuzman, M., Franelic, I. P., Pristas, I., Benjak, T. \& Dezeljin, J. D. 2010. Inequalities in Croatian pupils' unhealthy behaviours and health 
outcomes: role of school, peers and family affluence. European Journal of Public Health 21 (1), 122-128.

Smet, B., Maes, L. A., De Clercq, L., Haryanti, K. \& Djati Winarno, R. 1999.The health behaviour of schoolaged children in Semarang, Indonesia: methodological problems in cross-cultural research. Health Promotion International 14, 7-16.

Smith, C., Wold, B. \& Moore, L. 1992. Health behaviour research with adolescents: a perspective from the WHO cross-national Health Behaviour in School-aged Children study. Health Promotion Journal of Australia 2, 41-44.

Smith, G. D., Bartley M. \& Blane D. 1990. The Black report on socioeconomic inequalities in health 10 years on. British Medical Journal 301, 373-376.

Sperber, A. M., Devellis, R. F. \& Boehlecke, B. 1994. Cross-cultural translation: methodology and validation. Journal of Cross-Cultural Psychology 25, 501-524.

Starfield, B., Riley, A. W., Witt, W. P. \& Robertson, J. 2002. Social class gradients in health during adolescence. Journal of Epidemiology \& Community Health 56, 354-361.

Statistics Finland. 2011. Population structure. Retrieved on 19.3.2013 from http://www.stat.fi/til/vaerak/2010/index_en.html

Statistics Finland. 2012. Comprehensive schools had 539,500 pupils in 2012. Retrieved on 19.3.2013 fromhttp://www.stat.fi/til/pop/2012/pop_2012 _2012-11-5_tie_001_en.html

Statistical Finland. 2013. Income and consumption. Retrieved on 20.3.1013 from http://www.stat.fi/tup/suoluk/suoluk_tulot_en.html

Steinberg, L. D. 2008. Adolescence, $8^{\text {th }}$ Edition. New York, NY: McGraw-Hill.

Stice, E., Myers, M. G. \& Brown, S. A. 1998. A longitudinal grouping analysis of adolescent substance use escalation and de-escalation. Psychology of Addictive Behaviour 12, 14 -27.

Stronks, K. 1997. Socio-economic inequalities in health: individual choice or social circumstances? Rotterdam: Erasmus University Rotterdam.

Sun, P., Unger, J. B., Palmer, P., Ma, H., Xie, B., Sussman, S. \& Johnson, C. A. 2012. Relative income inequality and selected health outcomes in urban Chinese youth. Social Science \& Medicine 74, 84-91.

Tapert, S. F., Aarons, G. A., Sedlar, G. R. \& Brown, S. A. 2001. Adolescent substance use and sexual risk-taking behaviour. Journal of Adolescent Health 28, 181-189.

Tapert, S. F. \& Schweinsburg, A. D. 2005. The human adolescent brain and alcohol use disorders. In M. Galanter (Ed.) Recent developments in alcoholism: alcohol problems in adolescents and young adults: Epidemiology, neurobiology, prevention, treatment. New York: Kluwer Academic/Plenum Publishers 17, 177-197. 
Tator, C. H. (Ed.) 2008. Catastrophic Injuries in sports and recreation: causes and prevention : a Canadian study. $2^{\text {nd }}$ Edition. Tornoto: University of Toronto Press.

Tercyak, K. P., Goldman, P., Smith, A. \& Audrain, J. 2002. Interacting effects of depression and tobacco advertising receptivity on adolescent smoking. Journal of Pediatric Psychology 27 (2), 145-154.

The Social Issues Research Centre. 1998. Social and cultural aspects of drinking: a report to the European Commission. Oxford: The Social Issues Research Centre.

The World Bank. 2010. Country and lending groups. Retrieved on 20.3.2013 from http:/ / data.worldbank.org/about/country-classifications/country -and-lending-groups

Thomas, G., Reifman, A., Barnes, G. M. \& Farrel, M. P. 2000. Delayed onset of drunkenness as a protective factor for adolescent alcohol misuse and sexual risk taking: a longitudinal study. Deviant Behaviour 21 (2), 181-210.

Tian, G. Q. \& Liu, F. 2011. Is the demand for alcohol beverages in developing countries sensitive to price? Evidence from China. International Journal of Environmental Research and Public Health 8 (6), 2124-2131.

Tomar, S. L. 2003. Is use of smokeless tobacco a risk factor for cigarette smoking? The U.S. experience. Nicotine \& Tobacco Research 5 (4), 561-569.

Torsheim, T., Currie, C., Boyce, W. \& Samdal, O. 2006. Country material distribution and adolescents' perceived health: multilevel study of adolescents in 27 countries. Journal of Epidemiology and Community Health 60, 156-161.

Torsheim, T., Currie, C., Boyce, W., Kalnins, I., Overpeck, M. \& Haughland, S. 2004. Material deprivation and self-rated health: a multilevel study of adolescents from 22 European and North American countries. Social Science \& Medicine 59 (1), 1-12.

Torsheim, T., Wold, B. \& Samdal O. 2000. The Teacher and Classmate Support Scale: factor structure, test-retest reliability and validity in samples of 13and 15 year old adolescents. School Psychology International 21, 195-212.

Torsheim, T., Wold, B., Samdal, O. \& Haugland, S. 1997. Test-retest reliability of survey indicators measuring adolescent health and health behaviour. Bergen: Research Centre for Health Promotion, University of Bergen.

Tourangeau, R. \& Smith, T. W. 1996. Asking sensitive questions: the impact of data collection mode, question format, and question context. Public Opinion Quarterly 60, 275-304.

Tourangeau, R., Rips, L. J. \& Rasinski, K. 2000. The psychology of survey response. Cambridge: Cambridge University Press.

Townsend, P. 1987. Deprivation. Journal of Social Policy 16 (2), 147-164. 
Tripodi, S. J., Bender, K., Litschge, C. \& Vaughn, M. G. 2010. Intervention for reducing adolescent alcohol abuse: a meta analytic review. Archives of Pediatrics \& Adolescent Medicine 164 (1), 85-91.

Tucker, J. S., Orlando, M. \& Ellickson, P. L. 2003. Patterns and correlates of binge drinking trajectories from early adolescence to young adulthood. Health Psychology 22, 79-87.

Turner, C. F., Ku, L., Rogers, S. M., Lindberg, L. D., Pleck, J. H. \& Sonenstein, F. L. 1998. Adolescent sexual behaviour, drug use, and violence: increased reporting with computer survey technology. Science 280, 867-873.

Turner, L., Mermelstein, R. \& Flay, B. 2004. Individual and contextual influences on adolescent smoking. Annals of the New York Academy of Sciences 1024, 175-197.

Tuinstra, J., Groothoff, J. W., van den Heuvel, W. J. A. \& Post, D. 1998. Socioeconomic differences in health risk behaviour in adolescence. Do they exist? Social Science and Medicine 47, 67-74.

Tyas, S. L. \& Pederson, L. L. 1998. Psychosocial factors related to adolescent smoking: a critical review of literature. Tobacco Control 7, 409-420.

Tynjälä, J. 1999. Sleep habits, perceived sleep quality and tiredness among adolescents. A health behavioural approach. Doctoral thesis. Jyväskylä: University of Jyväskylä.

U.S. Department of Health and Human Services. 2004. The health consequences of smoking: a report of the Surgeon General. Washington: DHHS, Centres for Disease Control and Prevention, National Centre for Chronic Disease Prevention and Health Promotion, Office on Smoking and Health.

U.S. Department of Health and Human services. 2010. How tobacco smoke causes disease: the biology and behavioural basis for smoking-attributable disease. A Report of the Surgeon General. Rockville, MD: Office of the Surgeon General.

U.S. Department of Health and Human Services. 2012. Preventing tobacco use among youth and young adult: a report of the Surgeon General. Atlanta, GA: Department of Health and Human Services, Centres for Disease Control and Prevention, National Centre for Chronic Disease Prevention and Health Promotion, Office on Smoking and Health.

van Oort, F. V. van Lenthe, F. J. \& Mackenbach, J. P. 2005. Material, psychosocial, and behavioural factors in the explanation of educational inequalities in mortality in The Netherlands. Journal of Epidemiology \& Community Health 59 (3), 214-220.

Vereecken, C. A., Maes L. A. \& Bacquer, D. 2003a. The influence of parental occupation and the pupils' education level on lifestyle behaviours among adolescents in Belgium. Journal of Adolescent Health 34, 330-338. 
Vereecken, C. A. \& Maes L. A. 2003b. A Belgian study on the reliability and relative validity of the Health Behaviour in School-aged Children food frequency questionnaire. Public Health Nutrition 6, 581-588.

Vuori, M., Ojala, K., Tynjälä, J., Villberg, J., Välimaa, R., \& Kannas, L. 2005. Liikunta-aktiivisuutta koskevien kysymysten stabiliteetti WHOKoululaistutkimuksessa. [The reliability of physical activity survey items in the HBSC study]. Liikunta \& Tiede 42 (6), 39-46.

Välimaa, R., Kannas, L., Lahtinen, E., Peltonen, H., Tynjälä, J. \& Villberg, J. 2008. Finland: innovative health education curriculum and other investments for promoting mental health and social cohesion among children and young people. In Social cohesion for mental well-being among adolescents. Copenhagen: WHO Regional Office for Europe.

Walker, S. P., Wachs, T. D., Gardner, J. M., Lozoff, B., Wasserman, G. A. \& Pollitt, E. 2007. Child development: risk factors for adverse outcomes in developing countries. Lancet 369 (9556), 145-157.

Wang, J., Deng, X., Wang, J., Wang, X. \& Xu, L. 2009. Substance use, sexual behaviours, and suicidal ideation and attempts among adolescents: findings from the 2004 Guangzhou Youth Risk Behaviour Survey. Public Health 123, 116-121.

Wang, Q., Wei, Y. L., He, Y., Lu, R. \& Liao, J. 2011. Longitudinal study of smoking behavior of middle school students in three years in Chengdu. Chinese Journal of School Health 32, 1434-1435.

Wangenaar, A. C., Salois, M. J. \& Komro, K. A. 2009. Effects of beverage alcohol price and tax levels on drinking: a meta-analysis of 1003 estimates from 112 studies. Addiction 104 (2), 179-190.

Wardle, J., Robb, K. \& Johnson, F. 2002. Assessing socioeconomic status in adolescents: the validity of a home affluence scale. Journal of Epidemiology and Community Health 56, 595-599.

Wardle, J., Robb, K. A., Johnson, F., Griffith, J., Brunner, E., Power, C. \& Martin, T. 2004. Socioeconomic variation in attitudes to eating and weight in female adolescents. Health Psychology 23 (3), 275-282.

Warren, C. W., Riley, L., Asma, S., Eriksen, M. P., Green, L., Blanton, C., Loo, C., Batchelor, S. \& Yach, D. 2000. Tobacco use by youth: A surveillance report from the Global Youth Tobacco Survey project. Bulletin of the World Health Organization 78(7), 868-876.

Wechsler, H., Dowdall, G. W., Maenner, G., Gledhill-Hoyt, J. \& Lee, H. 1998. Changes in binge drinking and related problems among American college students between 1993 and 1997. Results of the Harvard School of Public Health College Alcohol Study. The Journal of American College Health 47, 57-68.

West, P. 1991. Rethinking the health selection explanation for health inequalities. Social Science \& Medicine 32, 373-384. 
West, P. \& Sweeting, H. 2004. Evidence on equalization in health in youth from the West of Scotland. Social Science \& Medicine 59 (1), 13-27.

West, P., Macintyre, S., Annandale, E. \& Hunt, K. 1990. Social class and health in youth: Findings from The West of Scotland Twenty-07 Study. Social Science \& Medicine 30 (6), 665-673.

West, P., Sweeting, H. \& Young, R. 2007. Smoking in Scottish youth: personal income, parental social class and the cost of smoking. Tobacco Control 16 (5), 329-335.

Wiehe, S. E., Garrison, M. M., Christakis, D. A., Ebel, B. E. \& Rivara, F. P. 2005. A systematic review of school-based smoking prevention trials with longterm follow-up. Journal of Adolescent Health 36 (3), 162-169.

Wijatkowski, S., Forgays, D. G., Wrzesniewski, K. \& Gorski, T. 1990. Smokingbehaviour and personality-characteristics in Polish adolescents. International Journal of the Addiction 25 (4), 363-373.

Wills, T. A., Sandy, J. M., Yaeger, A. M., Cleary, S. D. \& Shinar, O. 2001. Coping dimensions, life stress, and adolescent substance use: a latent growth analysis. Journal of Abnormal Psychology 110 (2), 309-323.

Woodward, A. \& Kawachi, I. 2000. Why reduce health inequalities? Journal of Epidemiology and Community Health 54, 923-929.

Woodward, M., Oliphant, J., Lowe, G. \& Tunstall-Pedoe, H. 2003. Contribution of contemporaneous risk factors to social inequality in coronary heart disease and all causes mortality. Preventive Medicine 36 (5), 561568.World Health Organization. 1999. Global Status Report on Alcohol. Geneva: World Health Organization.

World Health Organization. 2004a. Global Status Report on Alcohol. Geneva: World Health Organization.

World Health Organization. 2004b. The World Health Report 2004: changing history. Geneva: World Health Organization.

World Health Organization. 2005. WHO Framework Convention on Tobacco Control. Genera: World Health Organization.

World Health Organization. 2007. WHO Expert Committee on Problems Related to Alcohol Consumption. Second report. WHO Technical Report Series, No. 944. Geneva: World Health Organization.

World Health Organization. 2010. Global strategy to reduce harmful use of alcohol. Geneva: World Health Organization.

World Health Organization. 2011a. Fact sheet on alcohol. Retrieved on 28.1.2013 from http://www.who.int/mediacentre/factsheets/fs349/en/ index.html

World Health Organization. 2011b. Global status report on alcohol and health. Geneva: World Health Organization. 
World Health Organization. 2011c. Report on the global tobacco epidemic: warning about the dangers of tobacco. Geneva: World Health Organization.

World Health Organization. 2011d. Young people: health risks and solutions. Fact sheet No. 345. Retrieved on 27.2.2013 from http:/ / www.who.int/ mediacentre/factsheets/fs345/en/index.html

World Health Organization. 2012a. Fact sheet on tobacco. Retrieved on 28.1.2013 from http://www.who.int/mediacentre/factsheets/ fs339/en/ index.html

World Health Organization. 2012b. Country health profile: China. Retrieved on 28.1.2013 from http://www.who.int/gho/countries/chn.pdf

World Health Organization. 2013. Maternal, new born, child and adolescent health. Retrieved on 28.1.2013 from http://www.who.int/maternal_child _adolescent/topics/adolescence/dev/en/index.html

Wray-Lake, L., Maggs, J. L., Johnston, L. D., Bachman, J. G., O'Malley, P. M. \& Schulenberg, J. E. 2012. Associations between community attachments and adolescent substance use in nationally representative samples. Journal of Adolescent Health 51 (4), 325-331.

Xian, H., Scherrer, J. F., Madden, P. A., Lyons, M. J., Tsuang, M., True, W. R. \& Eisen, S. A. 2005. Latent class typology of nicotine withdrawal: genetic contributions and association with failed smoking cessation and psychiatric disorders. Psychological Medicine 35, 409-419.

Xian, H, Scherrer, J. F., Madden, P. A. Lyons, M. J., Tsuang, M., True, W. R. \& Eisen, S. A. 2003. The heritability of failed smoking cessation and nicotine withdrawal in twins who smoked and attempted to quit. Nicotine \& Tobacco Research 5 (2), 245-254.

Xing, Y., Ji, C. \& Zhang, L. 2006. Relationship of binge drinking and other health-compromising behaviours among urban adolescents in China. Journal of Adolescent Health 39, 495-500.

Xing, Y., Ji, C., Yang, X., Ji, H., Yang, L., Wang, Y. \& Zhang L 2002. The prevalence of addictive behaviours among middle school students in Shijingshan, Beijing. Chinese Journal of School Doctor 16, 684-686.

Yen, M. \& Lo, L. H. 2002. Examine test-retest reliability: an Intra-Class Correlation approach. Nursing Research 51 (1), 59-62.

Yeretzian, J. S. \& Afifi, R. A. 2009. 'It won't happen to me': the knowledgeattitude nexus in adolescent smoking. Journal of Public Health 31 (3), 354359.

Yu, C. Y. \& Muthén, B. 2002. Evaluation of model fit indices for latent variable models with categorical and continuous outcomes. Doctoral thesis. Los Angles: University of California at Los Angeles.

Zhang, H. \& Cai, B. 2003. The impact of tobacco on lung health in China. Respirology 8, 17-21. 
Zhang, J. F., Mauzerall, D. L., Zhu, T., Liang, S., Ezzati, M. \& Remais, J. V. 2010. Environmental health in China: progress towards clean air and safe water. Lancet 375, 1110-1119.

Zhou, L. 2009. Taxes of alcohol and cigarette in China. In Guangming Daily 25.6.2009. Retrieved on 4.3.2013 from http://news.xinhuanet.com/ politics /2009-06/25/content_11599561.htm

Zeigler, D. W., Wang, C. \& Yoast, R. A. 2005. The neurocognitive effects of alcohol on adolescents and college students. Preventive Medicine 40, 2332.

Øvretveit, J. (Ed.) 1998. Comparative and cross-cultural health research: a practical guide. Oxford: Radcliffe Medical Press. 
Appendix 1 English translation of survey items used in this study from the questionnaire of the HBSC linked project in China and the Finnish HBSC study 2006 (for 15year-olds)

1. Sex

$1 \square$ Boy $\quad 2 \square$ Girl

2. What class are you in? (In Finnish Survey the alternatives are $5^{\text {th }}, 7^{\text {th }}$ and $9^{\text {th }}$ grade)
$1 \square 5^{\text {th }}$ grade
$2 \square 6^{\text {th }}$ grade
$3 \square 7$ th grade
$4 \square 8^{\text {th }}$ grade
$5 \square 9^{\text {th }}$ grade
$6 \square 10^{\text {th }}$ grade
$7 \square 11^{\text {th }}$ grade

3. What month were you born?

$\begin{array}{llllllllllll}\text { Jan } & \text { Feb } & \text { Mar } & \text { Apr } & \text { May } & \text { Jun } & \text { Jul } & \text { Aug } & \text { Sep } & \text { Oct } & \text { Nov } & \text { Dec } \\ 1 \square & 2 \square & 3 \square & 4 \square & 5 \square & 6 \square & 7 \square & 8 \square & 9 \square & 10 \square & 11 \square & 12 \square\end{array}$

4. What year were you born? (In Finnish Survey the alternatives are from 1989 to 1996)

$\begin{array}{lllllllll}1991 & 1992 & 1993 & 1994 & 1995 & 1996 & 1997 & 1998 & 1999 \\ 1991 \square & 1992 \square & 1993 \square & 1994 \square & 1995 \square & 1996 \square & 1997 \square & 1998 \square & 1999 \square\end{array}$

9. What kind of place do you live in?

$1 \square$ City, in the centre $\quad 2 \square$ City, outside the centre

$3 \square$ Countryside, in the village centre $4 \square$ Countryside

11. Does your father go to work? If no, please answer 12 , otherwise move to 13
$1 \square$ Yes (Including farming)
$2 \square$ No
$3 \square$ I don't know
$4 \square$ I have no father or I don't see him

12. Why does not your father go to work? Please choose the most true answer $1 \square$ He is sick, retired or a student

$2 \square$ He is looking for a job

$3 \square$ He takes care of the family members or is full-time at home

$4 \square$ I don't know

13. Does your mother go to work? If no, please answer 14, otherwise move to 15
$1 \square$ Yes (Including farming)
$2 \square$ No
$3 \square$ I don't know
$4 \square$ I have no father or I don't see her

14. Why does not your father go to work? Please choose the most true answer $1 \square$ She is sick, retired or a student $2 \square$ She is looking for a job

$3 \square$ She takes care of the family members or is full-time at home $4 \square$ I don't know 
15. What is the highest level of education that your parents have? (Used in Chinese survey only)

\begin{tabular}{lcc} 
& Mother & Father \\
\hline 1. Under the primary school & $\square$ & $\square$ \\
\hline 2. Primary school & $\square$ & $\square$ \\
\hline 3. Secondary school & $\square$ & $\square$ \\
\hline 4. Upper secondary school & $\square$ & $\square$ \\
\hline 5. Junior college & $\square$ & $\square$ \\
\hline 6. University (Bachelor level) & $\square$ & $\square$ \\
\hline 7, Graduate or more & $\square$ & $\square$ \\
\hline
\end{tabular}

In the following questions, physical activity is any activity that increases your heart rate and makes you get out of breath some of the time, e.g. when training, playing games with friends on your way to school and or during physical education classes at school. Physical activity includes e.g. running, walking briskly, roller-skating, cycling, dancing, skateboarding, swimming, football, basketball, volleyball, table tennis and badminton etc.

For these next two questions, add up all the time you spend in physical activity each day.

21. Over the past 7 days, on how many days were you physically active for a total of at least 60 minutes per day?

$$
\begin{array}{lccccccc}
0 \square & 1 \square & 2 \square & 3 \square & 4 \square & 5 \square & 6 \square & 7 \square \\
0 \text { Day } & 1 & 2 & 3 & 4 & 5 & 6 & 7 \text { Days }
\end{array}
$$

22. Over a typical or usual week, on how many days are you physically active for a total of at least 60 minutes per day?

$$
\begin{array}{lrrrrrrl}
0 \square & 1 \square & 2 \square & 3 \square & 4 \square & 5 \square & 6 \square & 7 \square \\
\text { 0 Day } & 1 & 2 & 3 & 4 & 5 & 6 & 7 \text { Days }
\end{array}
$$

29. OUTSIDE SCHOOL CLASS: How OFTEN do you usually take physical exercise in your free time so that you lose your breath or sweat?
$1 \square$ Daily
$2 \square$ 4-6 times a week
$3 \square$ 2-3 times a week
$4 \square$ Once a week
$5 \square$ Once a month
$6 \square$ Less than once a month

7ロ Never

30. OUTSIDE SCHOOL CLASS: How many HOURS a week do you usually take physical exercise in your free time so that you lose your breath or sweat?
$1 \square$ None
$2 \square$ Approx. half an hour
$3 \square$ Approx. an hour
$4 \square$ Approx. 2-3 hours
5ロ Approx. 4-6 hours
$6 \square$ Seven hours or more 
34. How many hours a day do you usually watch TV or videos or DVDs in your free time?

Tick one box for school days and one for weekend, the same as 35-37.

\section{School days}

$1 \square$ None

$2 \square$ Approx. half an hour

$3 \square$ Approx. an hour a day

$4 \square$ Approx. 2 hours a day

$5 \square$ Approx. 3 hours a day

$6 \square$ Approx. 4 hours a day

$7 \square$ Approx. 5 hours a day

$8 \square$ Approx. 6 hours a day

$9 \square$ Approx. 7 hours a day or more
Weekend

$1 \square$ None

$2 \square$ Approx. half an hour

$3 \square$ Approx. an hour a day

$4 \square$ Approx. 2 hours a day

$5 \square$ Approx. 3 hours a day

$6 \square$ Approx. 4 hours a day

$7 \square$ Approx. 5 hours a day

$8 \square$ Approx. 6 hours a day

$9 \square$ Approx. 7 hours a day or more

35. How many hours a day do you usually use a computer for playing games or use console games (Playstation, Xbox, GameCube etc.) in your free time?

$\underline{\text { School days }}$

$1 \square$ None

$2 \square$ Approx. half an hour

$3 \square$ Approx. an hour a day

$4 \square$ Approx. 2 hours a day

$5 \square$ Approx. 3 hours a day

$6 \square$ Approx. 4 hours a day

$7 \square$ Approx. 5 hours a day

8ロApprox. 6 hours a day

$9 \square$ Approx. 7 hours a day or more
Weekend

$1 \square$ None

$2 \square$ Approx. half an hour

$3 \square$ Approx. an hour a day

$4 \square$ Approx. 2 hours a day

$5 \square$ Approx. 3 hours a day

$6 \square$ Approx. 4 hours a day

$7 \square$ Approx. 5 hours a day

8ロApprox. 6 hours a day

$9 \square$ Approx. 7 hours a day or more

36. How many hours a day do you usually use a computer for the following things in your free time: chatting online, internet, emailing, homework etc.?

$\underline{\text { School days }}$

$1 \square$ None

$2 \square$ Approx. half an hour

$3 \square$ Approx. an hour a day

$4 \square$ Approx. 2 hours a day

$5 \square$ Approx. 3 hours a day

$6 \square$ Approx. 4 hours a day

$7 \square$ Approx. 5 hours a day

8ロApprox. 6 hours a day

9ロApprox. 7 hours a day or more
Weekend

$1 \square$ None

$2 \square$ Approx. half an hour

$3 \square$ Approx. an hour a day

$4 \square$ Approx. 2 hours a day

$5 \square$ Approx. 3 hours a day

$6 \square$ Approx. 4 hours a day

$7 \square$ Approx. 5 hours a day

$8 \square$ Approx. 6 hours a day

$9 \square$ Approx. 7 hours a day or more 
37. How many hours a day do you usually spend doing your school homework out of school hours? (Including the homework you do in school and home; the homework also includes the extra homework you have to done for special training purpose etc.) (Used in Chinese survey only)

School days
$1 \square$ None
$2 \square$ Approx. half an hour
$3 \square$ Approx. an hour a day
$4 \square$ Approx. 2 hours a day
$5 \square$ Approx. 3 hours a day
$6 \square$ Approx. 4 hours a day
$7 \square$ Approx. 5 hours a day
$8 \square$ Approx. 6 hours a day
$9 \square$ Approx. 7 hours a day or more

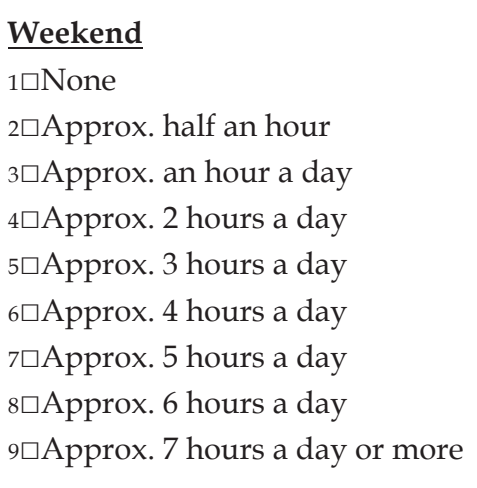

38. Does your family own a car? (a passenger car, a van or a lorry)
$1 \square$ No
$2 \square$ Yes, one
$3 \square$ Yes, two or more

39. Do you have your own room?
$1 \square$ No
$2 \square$ Yes

40. How many times over the last year did you travel somewhere on holiday with your family?
$1 \square$ Not at all
$2 \square$ Once
$3 \square$ Twice
$4 \square$ More than twice

41. How many computers do your family own? (Including laptop)
$1 \square$ None
$2 \square$ One
उम Two
$4 \square$ More than two

42. Have you ever smoked? (At least one cigarette, cigar or a pipeful)
$1 \square$ Yes
$2 \square$ No, move to 47

43. How often do you smoke at present?
$1 \square$ Every day
$2 \square$ Every week, but not daily
$3 \square$ Less than once a week
$4 \square$ I do not smoke

49. At present, how often do you have alcohol drinks like beer, wine or strong liquors? Also include the times when you only drink a very small amount.

\begin{tabular}{lccccc} 
& 1 1) & 2) & 3) & 4) \\
\cline { 2 - 5 } & Daily & $\begin{array}{c}\text { At least once a } \\
\text { week }\end{array}$ & $\begin{array}{c}\text { At least once a } \\
\text { month }\end{array}$ & Less & Never \\
\hline 1. Beer (Mild or strong) & $\square$ & $\square$ & $\square$ & $\square$ & $\square$ \\
2. Wine & $\square$ & $\square$ & $\square$ & $\square$ & $\square$ \\
3. Strong liquors & $\square$ & $\square$ & $\square$ & $\square$ & $\square$ \\
4. Mild alcoholic drinks & $\square$ & $\square$ & $\square$ & $\square$ \\
5. Other alcoholic drinks & $\square$ & $\square$ & $\square$ & $\square$ & $\square$ \\
\hline
\end{tabular}


50. Have you ever had so much alcohol that you have been really drunk?
$1 \square$ Never
$2 \square$ Yes, once
$3 \square$ Yes, 2-3 times
$4 \square$ Yes, 4-10 times
$5 \square$ Yes, more than 10 times

51. At what age did you do the following things for the first time? If you have not done the things mentioned here, choose 'Never'

\begin{tabular}{|c|c|c|c|c|c|c|c|}
\hline & Never & $\begin{array}{c}11 \text { years } \\
\text { old or less }\end{array}$ & $\begin{array}{c}12 \text { years } \\
\text { old }\end{array}$ & $\begin{array}{c}13 \text { years } \\
\text { old }\end{array}$ & $\begin{array}{c}14 \text { years } \\
\text { old }\end{array}$ & $\begin{array}{c}15 \text { years } \\
\text { old }\end{array}$ & $\begin{array}{l}16 \text { years } \\
\text { or older }\end{array}$ \\
\hline $\begin{array}{l}\text { 1. I drank alco- } \\
\text { hol (more than } \\
\text { a small amount) }\end{array}$ & $\square$ & $\square$ & $\square$ & $\square$ & $\square$ & $\square$ & $\square$ \\
\hline 2. I was drunk & $\square$ & $\square$ & $\square$ & $\square$ & $\square$ & $\square$ & $\square$ \\
\hline $\begin{array}{l}\text { 3. I smoked a } \\
\text { cigarette (more } \\
\text { than a puff) }\end{array}$ & $\square$ & $\square$ & $\square$ & $\square$ & $\square$ & $\square$ & $\square$ \\
\hline
\end{tabular}

62. What do you think of the financial situation of your family?

$1 \square$ My family is very well off financially

$2 \square$ My family is quite well off financially

$3 \square$ Average

$4 \square$ My family is not very well off financially

$5 \square$ My family is not at all well off financially

82. When do you usually go to bed if you have to go to school in the next morning?

\begin{tabular}{ccccccccccc}
$\begin{array}{c}\text { No later } \\
\text { than } \\
21.00\end{array}$ & 21.30 & 22.00 & 22.30 & 23.00 & 23.30 & 24.00 & 00.30 & 01.00 & 01.30 & $\begin{array}{c}02.00 \text { or } \\
\text { later }\end{array}$ \\
\hline $1 \square$ & $2 \square$ & $3 \square$ & $4 \square$ & $5 \square$ & $6 \square$ & $7 \square$ & $8 \square$ & $9 \square$ & $10 \square$ & $11 \square$ \\
\hline
\end{tabular}

83. When do you usually go to bed at weekends or during holidays?

\begin{tabular}{|c|c|c|c|c|c|c|c|c|c|c|c|c|c|c|}
\hline $\begin{array}{l}\text { No } \\
\text { later } \\
\text { than } \\
21.00\end{array}$ & 21.30 & 22.00 & 22.30 & 23.00 & 23.30 & 24.00 & 00.30 & 01.00 & 01.30 & 02.00 & 02.30 & 03.00 & 03.30 & $\begin{array}{c}04.00 \\
\text { or } \\
\text { later }\end{array}$ \\
\hline $1 \square$ & $2 \square$ & $3 \square$ & $4 \square$ & $5 \square$ & $6 \square$ & $7 \square$ & $8 \square$ & $9 \square$ & $10 \square$ & $11 \square$ & $12 \square$ & 13ロ & $14 \square$ & $15 \square$ \\
\hline
\end{tabular}

84. When do you usually wake up on school mornings?

\begin{tabular}{ccccccc}
$\begin{array}{c}\text { No later } \\
\text { than } \\
05.00\end{array}$ & 05.30 & 06.00 & 06.30 & 07.00 & 07.30 & $\begin{array}{c}08.00 \text { or } \\
\text { later }\end{array}$ \\
\hline $1 \square$ & $2 \square$ & $3 \square$ & $4 \square$ & $5 \square$ & $6 \square$ & $7 \square$ \\
\hline
\end{tabular}

85. When do you usually wake up on weekends?

\begin{tabular}{|c|c|c|c|c|c|c|c|c|c|c|c|c|c|c|}
\hline $\begin{array}{c}\text { No } \\
\text { later } \\
\text { than } \\
07.00 \\
\end{array}$ & 07.30 & 08.00 & 08.30 & 09.00 & 09.30 & 10.00 & 10.30 & 11.00 & 11.30 & 12.00 & 12.30 & 13.00 & 13.30 & $\begin{array}{c}14.00 \\
\text { or } \\
\text { later }\end{array}$ \\
\hline $1 \square$ & $2 \square$ & $3 \square$ & $4 \square$ & $5 \square$ & $6 \square$ & $7 \square$ & $8 \square$ & $9 \square$ & $10 \square$ & $11 \square$ & $12 \square$ & $13 \square$ & $14 \square$ & $15 \square$ \\
\hline
\end{tabular}




\title{
ORIGINAL PAPERS
}

I

TEST-RETEST RELIABLITY OF SELECETED ITEMS OF HEALTH BEHAVIOUR IN SCHOOL-AGED CHILDREN (HBSC) SURVEY QUESTIONNAIRE IN BEIJING, CHINA

\author{
by \\ Liu, Y., Wang, M., Tynjälä, J., Lv, Y., Villberg, J., Zhang, Z. \& Kannas, L. \\ 2010.
}

BMC Medical Research Methodology 10, 73.

Reprinted with kind permission by BioMed Central Ltd. 


\title{
Test-retest reliability of selected items of Health Behaviour in School-aged Children (HBSC) survey questionnaire in Beijing, China
}

Yang Liư, Mei Wang'2, Jorma Tynjälä', Yan Lv², Jari Villberg', Zhouyang Zhang ${ }^{3}$, Lasse Kannas'

\section{Abstract}

Background: Children's health and health behaviour are essential for their development and it is important to obtain abundant and accurate information to understand young people's health and health behaviour. The Health Behaviour in School-aged Children (HBSC) study is among the first large-scale international surveys on adolescent health through self-report questionnaires. So far, more than 40 countries in Europe and North America have been involved in the HBSC study. The purpose of this study is to assess the test-retest reliability of selected items in the Chinese version of the HBSC survey questionnaire in a sample of adolescents in Beijing, China.

Methods: A sample of 95 male and female students aged 11 or 15 years old participated in a test and retest with a three weeks interval. Student Identity numbers of respondents were utilized to permit matching of test-retest questionnaires. 23 items concerning physical activity, sedentary behaviour, sleep and substance use were evaluated by using the percentage of response shifts and the single measure Intraclass Correlation Coefficients (ICC) with 95\% confidence interval (Cl) for all respondents and stratified by gender and age. Items on substance use were only evaluated for school children aged 15 years old.

Results: The percentage of no response shift between test and retest varied from $32 \%$ for the item on computer use at weekends to $92 \%$ for the three items on smoking. Of all the 23 items evaluated, 6 items (26\%) showed a moderate reliability, 12 items (52\%) displayed a substantial reliability and 4 items (17\%) indicated almost perfect reliability. No gender and age group difference of the test-retest reliability was found except for a few items on sedentary behaviour.

Conclusions: The overall findings of this study suggest that most selected indicators in the HBSC survey questionnaire have satisfactory test-retest reliability for the students in Beijing. Further test-retest studies in a large and diverse sample, as well as validity studies, should be considered for the future Chinese HBSC study.
\end{abstract}

\section{Background}

Health behaviour of young people is a global concern. Currently, in China, a large range of problems concerning the health behaviour of the youth is emerging along with changes in lifestyle brought about by rapid economic development and globalization $[1,2]$. So far, only few national surveys concerning the health behaviour of the Chinese youth have been conducted. In addition to national level research, many studies which investigate a particular health behaviour, or a number of health

* Correspondence: yang.liu@jyu.fi

Research Centre for Health Promotion, Department of Health Sciences, University of Jyväskylä, Jyväskylä, Finland

Full list of author information is avalable at the end of the article behaviours and lifestyle traits of young people, have been done by Chinese researchers independently or through a collaborative project with foreign researchers [3-9]. Nevertheless, very few of them can give a comprehensive and comparable portfolio of health behaviour of young Chinese people.

Research exploring children's health behaviours and the factors that influence them are important for the development of effective health education and health promotion programs and policies for young people [10]. Many national and international level studies concerning young people's health behaviour have been conducted in recent decades. The Health Behaviour in School-aged Children (HBSC) study is among the first large-scale 
international surveys on adolescent health [11]. The participating countries, however, are only within Europe and North America. Since the HBSC study is a tool to examine health behaviour of young people, it is important to seek more international support to examine whether the survey instrument is useful in different continents and cultures. Therefore, for the development of the application of the HBSC study, it is significant and meaningful to expand its boarders in the future to include China, which has the largest population of school-aged children in the world.

Health behaviour is of crucial importance for the adolescents' health and their development [12-18]. It is important that the first step toward understanding young people's health is to obtain abundant and accurate data which represent the prevalence of health behaviour of the young people. Surveys are the most common methodological technique to understand and assess young people's health behaviour, especially in epidemiological studies where the use of a self-report questionnaire is often the only feasible method for the measurement of health behaviour such as physical activity [19]. Therefore, the reliability of the self-report questionnaire measuring health behaviour of adolescents is crucial since the low reliability may tend to mask the real prevalence and important relationships, which add difficulties or leads to the wrong development of rele vant policies, programmes and practices for the young people.

Meanwhile, the test-retest reliability can be influenced by many factors. From the viewpoint of information process of answering questions, two main components of those factors can be distinguished; that the first component is the interpretation or understanding of a question, such as the familiarity of content, complexity and ambiguity of an item, and the second one is the role of memory [20]. Random answers may be found for those items which involve unfamiliar knowledge, or are too complex to understand and therefore yield an uncertain answer, or are ambiguous, leading to variable responses [21]. In addition, it is also understandable that the memory may affect the retest response if the time interval between the test and the retest is short; normally the time interval of test-retest reliability studies is chosen from one week to five weeks. Besides the information processing factors mentioned above, the nature of the item being measured can also affect the test-retest reliability [22]. For instance, the rather stable behaviour, liking smoking, may show higher test-retest reliability than the fluctuated behaviour, such as bullying or injuries.

The reliability of some existing HBSC items have been assessed by a number of countries in recent years, for example, Torsheim and his colleagues investigated the test-retest reliability of 31 selected items in Norway which were used as the indicators in the HBSC study [23]. Later, more studies concerning a specific topic have been done, such as family affluence [24], diet [25], overweight and obesity [26], physical activity [27-29], symptoms [30], reasons for exercise [31], sleep [32] and school environment [33]. In general, the data from those above mentioned studies indicate that most items of the HBSC survey questionnaire had acceptable reliability.

However, more research should be conducted on the survey indicators in different countries and cultures to ensure the continuous improvement of the survey instrument. In order to provide recommendations and conduct revisions for the future Chinese HBSC study, the pilot study using the HBSC 2005/06 survey questionnaire was completed in the Beijing area in 2008. The purpose of this study, therefore, was to examine the test-retest reliability of selected indicators from the HBSC questionnaire measuring physical activity, sedentary behaviour, sleep, and substance use in a Chinese population.

\section{Methods}

\section{Sampling}

This test-retest study is one part of the pilot study for the Health Behaviour and Lifestyle Survey for Schoolaged Children in Beijing 2008 in which the HBSC 2005/ 06 survey questionnaire was used. One primary school and one secondary school were randomly chosen in Beijing to conduct the pilot study. Two classes in grade 6 (students aged around 11 years old) and two classes in grade 10 (students aged around 15 years old) were randomly drawn from the two sample schools. All the students $(n=139)$ in these four classes participated in Test 1. Of those respondents, all the students from one class in grade 6 and two classes in grade 10 completed the questionnaire Test 2. Students from one class in grade 6 did not participate in Test 2 due to the overlap of the school schedule and the survey. No significant difference of the characteristics was found in Test 1 between the class in grade 6 of which students participated in both Test 1 and $2(n=44)$ and the drop out class $(n=44)$ (Table 1). The final sample for the test-retest study, therefore, consisted of 95 students. The demographic characteristics of respondents are shown in Table 2 . The proportion of boys and girls was almost equal in the younger age group, but among the older age group, there were more boys than girls. The mean age of respondents did not differ between boys and girls in either age group.

\section{Questionnaire items}

The questionnaire used in this study was based on the mandatory and optional questions of the HBSC Protocol for $2001 / 02$ Survey [10] as well as the questionnaire 
Table 1 Pearson Chi-Square Tests for response of the participants in Test 1 between the participants in both Test 1 and Test 2 (aged 11 years old, $n=44$ ) and the non-participants in Test 2 (aged 11 years old, $n=44$ )

\section{Items}

MVPA in the last 7 days 0.35

MVPA in the usual week

VPA frequency

VPA time per week

Watching TV on school days

Watching TV at weekends

Playing PC or console games on school days

Playing PC or console games at weekends

Using PC on school days

Using $P C$ at weekends

Doing homework on school days

Doing homework at weekends

When go to bed on school days

When go to bed at weekends

When wake up on school mornings

When wake up at weekends

$P$ value

used in the Finnish HBSC Survey in 2006. The questionnaire was firstly translated from English into Chinese by two researchers independently and re-translated from Chinese into English to check the discrepancies by other professional translators. Finally, the questionnaire contained 102 questions and the same questionnaire was used in both the test and the retest. Of those items, 23 items concerning physical activity (4 items), sedentary behaviour ( 8 items), sleep ( 4 items) and substance use (7 items) were evaluated in this test-retest study. The detailed information of items and their response alternatives can be found in Table 3 .

Data collection procedure

The test was administered by one researcher from th China Institute of Sport Science (CISS) and one class teacher from the school during an ordinary class hour. The students were instructed how to fill in the questionnaire by the researcher and they were not informed about the forthcoming retest. Three weeks later the retest was conducted through an identical procedure. Al students participating in the test and retest were asked

Table 2 Demographic characteristics of respondents

\begin{tabular}{|c|c|c|c|c|c|c|c|c|c|c|c|c|}
\hline & \multicolumn{4}{|c|}{ Test 1} & \multicolumn{4}{|c|}{ Test 2} & \multicolumn{4}{|c|}{ Age (years and decimals) of respondents answered in both tests } \\
\hline & \multicolumn{2}{|c|}{11} & \multicolumn{2}{|c|}{15} & \multicolumn{2}{|c|}{11} & \multicolumn{2}{|c|}{15} & \multicolumn{2}{|c|}{11} & \multicolumn{2}{|c|}{15} \\
\hline & $\mathrm{n}$ & $\%$ & $n$ & $\%$ & $n$ & $\%$ & $\mathrm{~N}$ & $\%$ & Mean & SD & Mean & SD \\
\hline Boys & 41 & 46.6 & 31 & 60.8 & 20 & 45.5 & 31 & 60.8 & 11.79 & 0.30 & 15.81 & 0.34 \\
\hline Girls & 47 & 53.4 & 20 & 39.2 & 24 & 54.5 & 20 & 39.2 & 11.63 & 0.37 & 15.78 & 0.30 \\
\hline Total & 88 & 100 & 51 & 100 & 44 & 100 & 51 & 100 & 11.70 & 0.35 & 15.80 & 0.32 \\
\hline
\end{tabular}

to write their student Identity number on the questionnaire to permit matching the test and retest questionnaires. Student's participation in the test and retest was totally voluntary and the questionnaire, as well as the student Identity number, can only be accessed by the researcher. Students were also informed that only the researcher will read their answers. Verbal consent was sought from all the participants, the head teachers of the classes, and the principle of the school. The test and retest were done at the end of October and at the middle of November in 2008. The study was approved by the ethics committee of CISS and the Research Centre for Health Promotion at the University of Jyväskylä.

\section{Data analyses}

All data from test and retest studies were entered by Epidata 3.1 with double entry and validation and analyzed by Statistical Package for the Social Sciences, version 15.0 (SPSS, Inc., Chicago, Illinois, US). Overall stability rate of items were given by the proportion of subjects showing no response shift on the item between test and retest. The frequency of response shifts of 1,2 and 3 or more categories were also computed. The test-retest reliability of all selected items were estimated using the single measure of Intraclass Correlation Coefficients (ICC) which were computed as devised by Shrout and Fleiss [34], through case 2 (using a two-way random model with an absolute agreement type), with $95 \%$ confidence interval (CI), for all respondents and stratified by gender and age. These values were considered significantly different if their $95 \%$ confidence intervals (CIs) did not overlap. According to Landis and Koch [35], the strength of test-retest agreement for ICC is classified as follows: below 0.20 is poor; 0.21 to 0.40 shows a fair agreement; 0.41 to 0.60 indicates a moderate degree of agreement; 0.61 to 0.80 means substantial agreement; and 0.81 to 1 indicates almost perfect agreement. These classifications were used to interpret the results. The items about substance use were evaluated only for the adolescents aged 15 years old due to the absence in this behaviour among 11 years-old respondents.

\section{Results}

The proportions of no response shift between test and use at weekends, to $92 \%$ for the three items on smoking retest varied from $32 \%$ for the item measuring computer 
Table 3 The selected items and response alternatives of HBSC survey questionnaire used in test-retest study Response Alternative

\section{Physical Activity}

1. Over the past 7 days, on how many days were you physically active for a total 0 day; $1 ; 2 ; 3 ; 4 ; 5 ; 6 ; 7$ days. of at least 60 minutes per day?

2. Over a typical or usual week, on how many days are you physically active for a 0 day; $1 ; 2 ; 3 ; 4 ; 5 ; 6 ; 7$ days. total of at least 60 minutes per day?

3. OUTSIDE SCHOOL CLASS: How OFTEN do you usually take physical exercise in your free time so that you lose your breath or sweat?

4. OUTSIDE SCHOOL CLASS: How many HOURS a week do you usually take physical exercise in your free time so that you lose your breath or sweat? Sedentary Behaviour

How many hours a day do you usually do the following things in your free time?

5. Watch TV or videos or DVDs on school days

6. Watch TV or videos or DVDs at weekends

7. Use a computer for playing games or use console games on school days

8. Use a computer for playing games or use console games at weekends

9. Use a computer for: chatting online, internet, emailing, homework etc on school days

10. Use a computer for: chatting online, internet, emailing, homework etc at weekends

11. Spend doing your school homework out of school hours on school days

12. Spend doing your school homework out of school hours at weekends

Sleep

13. When do you usually go to bed if you have to go to school in the next morning?

14. When do you usually go to bed at weekends or during holidays?

15. When do you usually wake up on school mornings?

16. When do you usually wake up at weekends?

Daily; 4-6 times a week; 2-3 times a week:

None: Approx hat an houri Approx an hour: Approx. $2-3$ hours; Approx 4-6 hours. Seven hours or more.

None; Approx. half an hour

Approx. an hour; Approx. 2 hours: Approx. 3 hours; Approx. 4 hours;

Approx. 5 hours; Approx. 6 hours;

Approx. 7 hours or more.

Risk Behaviour: substance us

17. Have you ever smoked?

18. How often do you smoke at present?

19. How many cigarettes, pipefuls or cigars have you smoked until now? At present, how often do you drink...?

20. Beer

21. Wine

22. Strong liquors

23. Have you ever had so much alcohol that you have been really drunk?

No later than 21:00: 21:30: 22:00; 22:30; 23:00: 23:30: 24:00: 00:30; 01:00; 01:30; 02:00 or later.

No later than 21:00; $21: 30 ; 22: 00 ; 22: 30 ; 23: 00 ; 23: 30 ; 24: 00 ; 00: 30$

01:00; 01:30; 02:00; 02:30; 03:00; 03:30; 04:00 or later.

No later than 05:00; 05:30; 06:00; 06:30; 07:00; 07:30; 08:00 or later. No later than 07:00; 07:30; 08:00; 08:30; 09:00; 09:30; 10:00; 10:30; 11:00; 11:30; 12:00; 12:30; 13:00; 13:30; 14:00 or later

Yes; No.

Every day; Every week, but not daily; Less than once a week; I do not smoke.

None; One; Approx. 2-50; More than 50.

Daily; At least once a week; At least once a month;

Rarely; Never.

Never; Yes, once; Yes, 2-3 times; Yes, 4-10 times; Yes, more than 10 times.

HBSC $=$ Health Behaviour in School-aged Children

behaviour. At least $68 \%$ of the respondents gave an answer in the same or an adjacent category for all selected indicators (Figure 1).

The values of ICC for all respondents were stratified by gender and age. These are shown in Tables 4 and 5 . Overall, the values of ICC of the selected items ranged from 0.33 to 0.85 , with the lowest value for the item regarding using a computer on school days, and the highest value for items on how many cigarettes ever smoked and pertaining to the question "have you ever been drunk?" Of all the 23 items evaluated in this study, according to Landis and Koch divisions of agreement

[35], 6 items (26\%) showed a moderate reliability, 12 items $(52 \%)$ displayed a substantial reliability and 4 items $(17 \%)$ indicated almost perfect reliability. By gender, the values of ICC varied from 0.19 to 0.96 for girls and 0.42 to 0.85 for boys. The items of the highest and lowest ICC for girls are not consistent with the items for boys. By age groups, ICC ranged from 0.38 to 0.86 for 11 year-old respondents and 0.16 to 0.85 for 15 year-old respondents.

\section{Physical activity}

The reliability of the four items assessing Moderate to Vigorous Physical Activity (MPVA) and Vigorous 


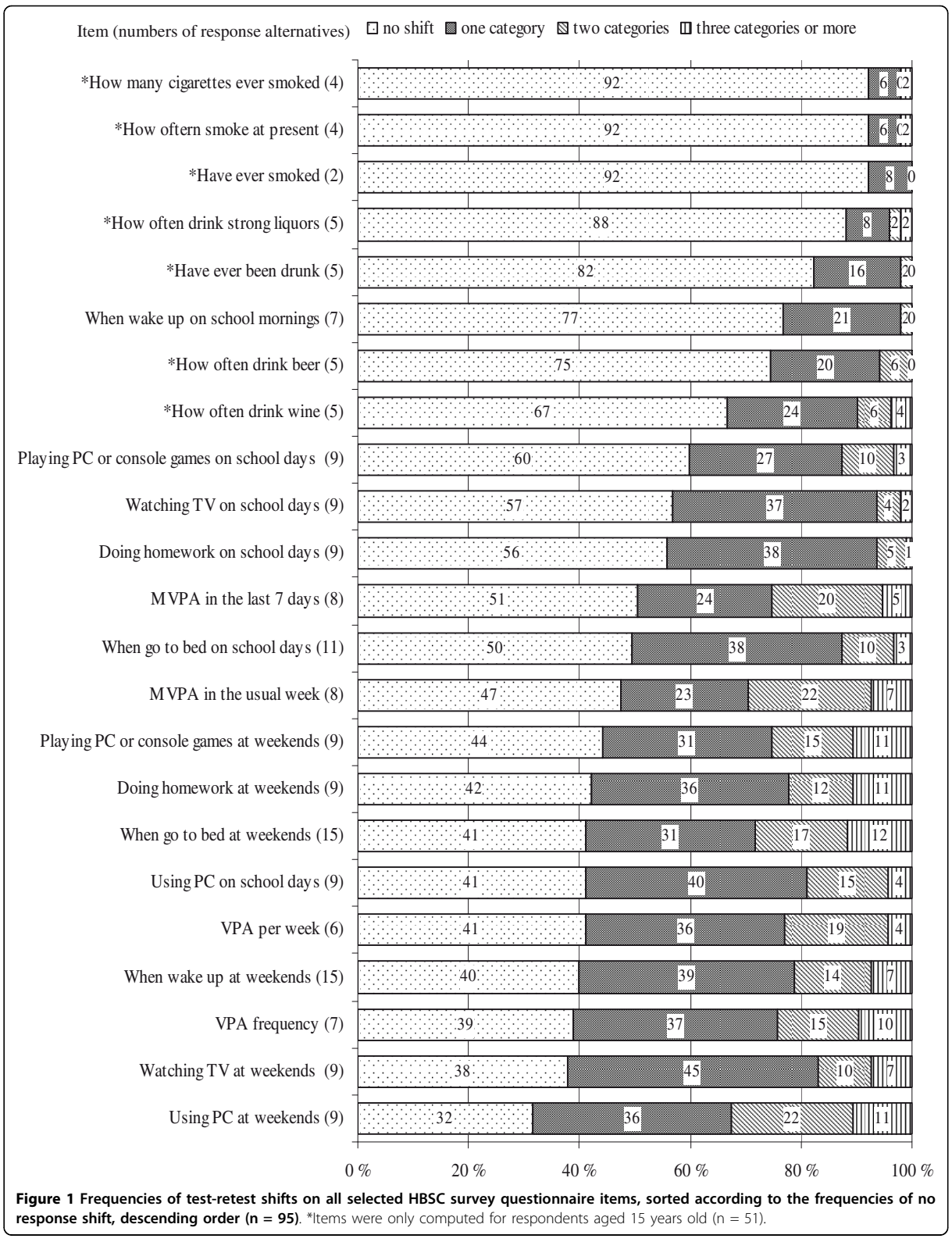


Table 4 ICC for HBSC survey questionnaire items about physical activity, sedentary behaviour and sleep by gender and age $(n=95)$

\begin{tabular}{|c|c|c|c|c|c|c|c|c|c|c|}
\hline \multirow[b]{2}{*}{ Items } & \multicolumn{2}{|c|}{ All $(n=95)$} & \multicolumn{2}{|c|}{ Girls $(n=44)$} & \multicolumn{2}{|c|}{ Boys $(n=51)$} & \multicolumn{2}{|c|}{11 years $(n=44)$} & \multicolumn{2}{|c|}{15 years $(n=51)$} \\
\hline & ICC & $95 \% \mathrm{Cl}$ & ICC & $95 \% \mathrm{Cl}$ & ICC & $95 \% \mathrm{Cl}$ & ICC & $95 \% \mathrm{Cl}$ & ICC & $95 \% \mathrm{Cl}$ \\
\hline \multicolumn{11}{|l|}{ Physical Activity } \\
\hline MVPA in the last 7 days & 0.82 & $0.74-0.88$ & 0.87 & $0.77-0.93$ & 0.79 & $0.66-0.88$ & 0.81 & $0.67-0.89$ & 0.79 & $0.66-0.88$ \\
\hline MVPA in the usual week & 0.74 & $0.64-0.82$ & 0.76 & $0.60-0.86$ & 0.73 & $0.57-0.83$ & 0.77 & $0.61-0.87$ & 0.71 & $0.54-0.82$ \\
\hline VPA frequency & 0.68 & $0.55-0.77$ & 0.73 & $0.56-0.85$ & 0.61 & $0.41-0.76$ & 0.72 & $0.54-0.84$ & 0.62 & $0.42-0.76$ \\
\hline VPA time per week & 0.57 & $0.42-0.66$ & 0.66 & $0.45-0.80$ & 0.50 & $0.27-0.68$ & 0.58 & $0.35-0.75$ & 0.56 & $0.34-0.72$ \\
\hline \multicolumn{11}{|l|}{ Sedentary Behaviour } \\
\hline Watching TV on school days & 0.72 & $0.61-0.81$ & $0.91^{*}$ & $0.83-0.95$ & 0.51 & $0.28-0.69$ & $0.86^{+}$ & $0.76-0.92$ & 0.57 & $0.36-0.73$ \\
\hline Watching TV at weekends & 0.74 & $0.63-0.83$ & 0.77 & $0.62-0.87$ & 0.70 & $0.53-0.82$ & 0.80 & $0.66-0.89$ & 0.68 & $0.49-0.80$ \\
\hline Playing PC or console games on school days & 0.54 & $0.38-0.67$ & 0.56 & $0.32-0.73$ & 0.53 & $0.30-0.70$ & 0.56 & $0.32-0.73$ & 0.52 & $0.29-0.70$ \\
\hline Playing PC or console games at weekends & 0.69 & $0.57-0.78$ & $0.47^{*}$ & $0.20-0.67$ & 0.83 & $0.72-0.90$ & 0.79 & $0.64-0.88$ & 0.57 & $0.35-0.73$ \\
\hline Using PC on school days & 0.33 & $0.14-0.50$ & 0.19 & $-0.11-0.46$ & 0.45 & $0.20-0.64$ & 0.38 & $0.10-0.60$ & 0.28 & $0.01-0.51$ \\
\hline Using PC at weekends & 0.50 & $0.33-0.64$ & 0.37 & $0.09-0.60$ & 0.58 & $0.36-0.73$ & $0.83^{+}$ & $0.71-0.90$ & 0.16 & $-0.12-0.41$ \\
\hline Doing homework on school days & 0.78 & $0.68-0.85$ & 0.81 & $0.68-0.89$ & 0.74 & $0.59-0.85$ & 0.75 & $0.59-0.86$ & 0.73 & $0.57-0.84$ \\
\hline Doing homework at weekends & 0.73 & $0.62-0.82$ & 0.69 & $0.49-0.82$ & 0.76 & $0.62-0.86$ & 0.54 & $0.29-0.72$ & 0.79 & $0.65-0.87$ \\
\hline \multicolumn{11}{|l|}{ Sleep } \\
\hline When go to bed on school days & 0.75 & $0.65-0.83$ & 0.76 & $0.60-0.86$ & 0.73 & $0.57-0.84$ & 0.68 & $0.48-0.81$ & 0.60 & $0.39-0.75$ \\
\hline When go to bed at weekends & 0.64 & $0.50-0.67$ & 0.64 & $0.43-0.79$ & 0.62 & $0.41-0.76$ & 0.51 & $0.26-0.70$ & 0.58 & $0.36-0.74$ \\
\hline When wake up on school mornings & 0.77 & $0.68-0.84$ & 0.79 & $0.65-0.88$ & 0.76 & $0.62-0.86$ & 0.81 & $0.68-0.89$ & 0.73 & $0.57-0.84$ \\
\hline When wake up at weekends & 0.83 & $0.75-0.88$ & 0.82 & $0.69-0.90$ & 0.84 & $0.73-0.90$ & 0.85 & $0.74-0.92$ & 0.78 & $0.65-0.87$ \\
\hline
\end{tabular}

$\mathrm{ICC}=$ Single measure intraclass correlation coefficient

HBSC = Health Behaviour in School-aged Children

* Significant difference between genders $(\mathrm{p}<0.05)$

" Significant difference between age groups $(p<0.05)$

Physical Activity (VPA) ranged from moderate (ICC = $0.57)$ to almost perfect agreement $(\mathrm{ICC}=0.82)$ in general. The lowest reliability was found in the item measuring VPA time per week and the highest reliability in the item relating to MVPA in the last 7 days. No statistically significant differences were found either by gender or by age group, though the ICC value may differ

Table 5 ICC for HBSC survey questionnaire items about substance use of 15-year-old children by gender $(n=51)$

All $(n=51) \quad$ Girls $(n=20) \quad$ Boys $(n=31)$ $\begin{array}{lllllll}\text { ICC } & \mathbf{9 5 \%} \mathrm{Cl} & \mathrm{ICC} & \mathbf{9 5 \%} \mathrm{Cl} & \mathrm{ICC} & \mathbf{9 5 \%} & \end{array}$

\begin{tabular}{lllllll}
\hline Have ever smoked & 0.75 & $0.60-0.85$ & $\mathrm{NA}$ & $/$ & 0.63 & $0.36-0.80$ \\
How often smoke at & 0.50 & $0.27-0.68$ & $\mathrm{NA}$ & $/$ & 0.48 & $0.15-0.71$
\end{tabular}

present

$\begin{array}{lllllll}\text { How many cigarettes } & 0.85 & 0.75-0.91 & \text { NA } & \text { / } & 0.81 & 0.64-0.90\end{array}$ ever smoked

How often drink beer $\quad 0.80 \quad 0.67-0.88 \quad 0.96 \quad 0.90-0.98 \quad 0.82 \quad 0.66-0.91$

$\begin{array}{lllllll}\text { How often drink wine } & 0.53 & 0.30-0.70 & 0.70 & 0.39-0.87 & 0.42 & 0.09-0.67\end{array}$ $\begin{array}{lllllll}\text { How often drink } & 0.44 & 0.19-0.64 & 0.76 & 0.49-0.90 & 0.64 & 0.37-0.81\end{array}$ strong liquors

Have ever been drunk $\quad 0.85 \quad 0.76-0.91 \quad 0.83 \quad 0.61-0.93 \quad 0.85 \quad 0.72-0.93$

$\mathrm{ICC}=$ Single measure intraclass correlation coefficient

$\mathrm{HBSC}=$ Health Behaviour in School-aged Children

NA: Not applicable due to lack of variance

Sedentary behaviour

Of the eight items examining the sedentary behaviours, seven of them showed a moderate to a substantial agreement. The question inquiring about using a computer on school days was the only item which indicated a fair agreement, and expressed the lowest value of ICC (0.33) for all respondents among all the selected items in this study. Significant gender differences were found in items on watching TV on school days and playing computer or console games at weekends $(\mathrm{p}<0.05)$. Meanwhile, significant age differences were found in items on watching TV on school days and using a computer at weekends $(\mathrm{p}<0.05)$

Sleep

All items on sleep patterns demonstrated at least substantial reliability, especially for the item on when children wake up at weekends, for which the reliability is almost perfect $(\mathrm{ICC}=0.83$ ). On the contrary, the lowest value of ICC was found for the item on when children go to bed at weekends (ICC $=0.64$ ). There were no gender and age differences in these items.

\section{Substance use}

The items on substance use were evaluated only for students aged 15 years old. Four items indicated a 
substantial to almost perfect reliability and the values of ICC varied from 0.75 to 0.85 . The other three items showed at least moderate reliability and the lowest reliability was exhibited by the question of how often do you drink strong liquors (ICC $=0.44$ ). None of the girls in this study reported they have ever smoked, so this constant result lead to the value of ICC for three items on smoking not applicable due to lack of variance.

\section{Discussion}

Overall, the test-retest reliability results showed moderate to almost perfect agreement for most of the items except for one item about sedentary behaviour. Finding in our study suggest that these indicators are reliable to measure health behaviour of school-aged children in Beijing. A few gender and age group differences were observed in the reliability of some indicators measuring sedentary behaviour among respondents.

The reliability of items measuring physical activity in this study indicated that both MVPA and VPA items are reliable measures of physical activity, which is a similar finding compared to previous studies $[23,27,29,36,37]$. One interesting finding from our study was that the lowest reliability was found for the item measuring VPA time per week (ICC $=0.57$ ), whereas usually VPA is more easily recalled than MVPA in adults. One possible reason for this might be that young people are in a period of trying different new sports and exercise. Therefore, compared to VPA, MVPA on a daily basis is more stable, although it is more difficult to recall. Vuori and his colleagues also reported similar results concerning the test-retest reliability of HBSC survey items measuring MVPA and VPA [29]. When considering items measuring physical activity, another interesting observation was that no age group differences were found in our study whereas some earlier studies have reported that the reliability of self-reported physical activity indicators generally improve with age $[27,28,37]$. However, it should be noted that the lack of age effects could partly reflect low statistical power to detect differences in coefficients. In addition, gender differences were not found in this study, unlike the findings of Rangul and his colleagues in their study [28], which showed items about physical activity in the HBSC questionnaire were more reliable for girls. A possible explanation for the non-existent difference within gender and age groups may be the fact that since 2007 the 'Sunshine Project' was carried out in all primary schools and high schools in China to ensure each student participates in physical activity at least one hour per day. This results in the students having a clear consciousness concerning physical activity participation so that the behaviour can be reported accurately no matter the age and gender. However this conclusion should be viewed with caution since the sample size of this study is rather small.

Similarly to the earlier study of Hardy and his colleagues [38], the items about sedentary behaviour in this study showed acceptable reliability. However, the reliability of items related to sedentary behaviour is lower than other behaviours. A striking result is that the item on "using a computer on school days" showed the lowest value of ICC (0.33) in all selected questions. One possible reason for this finding is that students probably do not have the same possibility to access the computer at school on school days because of the different school curriculum and content of study in different school weeks. In general, the reasons for the low value of ICC are mainly due to poor reliability of answering the item or the behaviour which the item measured is not very stable between the test and retest. For this item, the poor agreement was most likely due to the rather unstable behaviour caused by the school schedule which influenced the students' use of the computer on school days. The results also revealed a difference between age and gender groups, younger students and girls tended to be more reliable than older students and boys for several items on sedentary behaviour. One exception that should be pointed out is for the item inquiring about "playing computer or console games at weekends", boys are more reliable than girls probably because playing computer or console games is predominately a boys' activity, and girls' value is different, so that they might report inaccurately.

Normally, for the self-report measures, the more response alternatives used, the more reliability is found. It is not surprising that at least substantial reliability was revealed in questions asking about sleeping habits since at least seven to fifteen response alternatives were recruited for them. Added to that, since sleep is a regular daily activity, knowledge and salience of sleep would be high. These results were very similar to the findings of Tynjälä's study [32]. It is evident for students that they have to wake up at a certain time in order to attend school on school days. Consequently, the items measuring sleeping behaviour are stable to some extent.

The study showed that items relating to smoking and alcohol use for 15 year-old students have a good reliability which is not surprising, as the finding is similar to previous studies $[39,40]$. An explanation for this is the fact that substance use displays a certain degree of cross-time stability, and therefore it can be recalled more reliably than other health behaviours [41]. In addition, the salience of smoking and alcohol use might be higher compared to other health behaviours, since most students need to an attitude towards such behaviours. Normally smoking behaviour would not change in the short term, but considering the students smoking is 
absolutely prohibited in Chinese schools and by most of their parents, it is understandable that the present smoking frequency of students who smoked may differ in terms of the different possibility to access cigarettes and smoke them. Another notable finding is that when students were asked about how often they drink beer, wine and strong liquors, the answers for wine and strong liquors are not as stable as for beer. The underlying reason for this is that many students have no clear definition of wine and strong liquors because compared to western countries, wine is rather seldom drunk for the masses in China, and the diversity of Chinese strong liquors makes students' recall consumption unreliably compared to beer. Accordingly, these two items should be considered for revision or addition of more reference explanations.

As a part of the pilot study for the Health Behaviour and Lifestyle Survey for School-aged Children in Beijing 2008 , the test-retest study was conducted during the normal school class. None of the students in the sample classes refused to fill in the questionnaire and all respondents could complete the questionnaire within one school hour (45 minutes). No questions or more interpretations were asked about the items used in the questionnaire during the data collection. Those indicators measuring health behaviour in the survey questionnaire proved to be understandable and acceptable to the school-aged children in Beijing.

Although it is the first assessment of the test-retest reliability of items related to several indicators measuring health behaviour used in the HBSC survey questionnaire in a Chinese population, this study has several limitations. First, the sample size for the test-retest study is small and the two sampled schools both come from the urban area of Beijing. For a country like China, when social economic status and culture background are taken into account, it is challenging to interpret the findings without a large and diverse sample. Second, reliability is a necessary characteristic of a valid selfreport measure, but it is not sufficient to ensure the validity of questions. This study, however, did not examine the validity of survey indicators. Furthermore, qualitative study on the acceptability and reproducibility of the HBSC survey questionnaire is lacking in our study. Finally, to support using the HBSC survey questionnaire in a Chinese population, and in a future possible China HBSC study, more work should be encouraged to assess both reliability and validity of the HBSC survey questions among Chinese adolescents.

\section{Conclusions}

This study represents the first assessment of the testretest reliability of items, concerning physical activity, sedentary behaviour, sleep and substance use, from the
HBSC survey questionnaire, in a Chinese population. The overall findings of this study suggest that most selected items in the HBSC survey questionnaire have satisfactory test-retest reliability for school-aged children in Beijing urban area. Despite the limitations, this study provided valuable information on feasibility and reliability of the HBSC survey questionnaire for the schoolaged children in Beijing urban area. Further studies in larger and more diverse samples, as well as validity studies should be considered in both urban and rural areas for the future Chinese HBSC study.

\section{Acknowledgements}

The authors would like to thank Ms. Lanmin Xiao from the Beijing Experimental School of Xicheng District and Ms. Jing Tian from Beijing Academy of Educational Sciences for helping to organize and conduct the field work. The authors also would like to thank Mr. Michael Ormshaw for checking the language and revising the manuscript. As a part of joint research project, this study was supported by China Institute of Sport Sciences (CISS) and the Research Centre for Health Promotion at the University of Jyväskylä. The data collection was funded by CISS and the first author was supported by grants from the Juho Vainion Foundation and the
University of Jyväskylä.

\section{Author details}

Research Centre for Health Promotion, Department of Health Sciences, University of Jyväskylä, Jyväskylä, Finland. 'Mass Sports Research Centre, China Institute of Sport Science, Beijing, China. Department of Sport and Health Science, Nanjing Institute of Physical Education, Nanjing, China.

\section{Authors' contribution}

All authors have read and approved the manuscript. YL, first author, made a substantial contribution to analyze data and write the original manuscript. MW contributed leading and designing the data collection, discussions an comments on the draft. J and LK commented and revised the draft cougrecting

Competing interests

The authors declare that they have no competing interests.

Received: 26 March 2010 Accepted: 10 August 2010

Published: 10 August 2010

References

Du S, Lu B, Zhai F, Popkin BM: A new stage of the nutrition transition in China. Public Health Nutr 2002, 51(1A):169-74.

General Sport Administration of China: Report on the Second National Physical Fitness Surveillance. Beijing: People's Sport Publishing Hous

3. Cheng TO: Teenage smoking in China. J Adolesc 1999, 22:607-20. Johnson CA, Palmer PH, Chou CP, Pang Z, Zhou D, Dong L, Xlang H, Yang $T$, anin: The

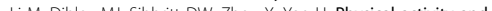
sedentary behaviour in adolescents in Xi'an City, China. J Adolesc Health 2007, 41:99-101.

6. Tudor-Locke C, Ainsworth BE, Adair LS, Du S, Popkin BM: Physical activity and inactivity in Chinese school-aged youth: the China Health and Nutrition Survey. Int J Obesity 2003, 27:1093-9.

7. Unger JB, Li Y, Anderson JC, Gong J, Chen X, Li C, Trinidad DR, Tran NT, LO AT. Stressful life events among adolescents in Wuhan, China: Associations with smoking, alcohol use and depressive symptoms. Int J Behav Med 2001, 8:1-18. 
8. Xing Y, Ji C, Zhang L: Relationship of binge drinking and other healthcompromising behaviours among urban adolescents in China. J Adoles Health 2006, 39:495-500

9. Zhu L. Petersen PE, Wang HY, Bian JY, Zhang BX: Oral health knowledge attitudes and behaviour of children and adolescents in China. Int Dent 2003, 53:289-98

10. Currie C, Samdal O, Boyce W, Smith R, (Eds): Health Behaviour in Schoolaged Children: a WHO Cross-National Study (HBSC), Research Protocol for the 2001/2002 Survey. Edinburgh: Child and Adolescent Health Research Unit (CAHRU), University of Edinburgh 2001.

11. Roberts C, Currie C, Samdal O, Currie D, Smith R, Maes L: Measuring the health and health behaviours of adolescents through cross-national survey research: recent developments in the Health Behaviour in School-aged Children (HBSC) study. J Pub Health 2007, 15:179-86.

12. Biddle SJ, Gorely T, Stensel DJ: Health-enhancing physical activity and sedentary behaviour in children and adolescents. J Sports Sci 2004. 22:679-701

13. Blair SN, LaMonte MJ, Nichaman MZ: The evolution of physical activity recommendations: how much is enough? Am I Clin Nutr 2004, 79(5):913-20

14. Rennie KL, Johnson L, Jebb SA: Behavioural determinants of obesity. Best Pract Res Clin Endocrinol Metab 2005, 19(3):343-58.

15. World Health Organization: Global Status Report on Alcohol Geneva: World Health Organization 2004

16. Mitru G, Millrood DL, Mateika JH: The impact of sleep on learning and behaviour in adolescents. Teach Coll Rec 2002, 104:704-26.

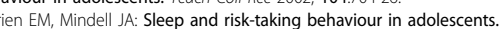
Behav Sleep Med 2005, 3:113-33.

18. Liu X, Liu L, Owens JA, Kaplan DL: Sleep patterns and sleep problems among schoolchildren in the United States and China. Pediatrics 2005, 115(1):241-9.

19. Kohl HW, Fulton JE, Casperson CJ: Assessment of physical activity among children and adolescents: a review and synthesis. Prev Med 2000, 31(Suppl):554-576

20. Otter ME, Mellenbergh GJ, Glopper KD: The relation between informationprocessing variables and test-retest stability for questionnaire items. $\int$ Educ Meas 1995, 32(2):199-216

21. Tourangeau R, Rips L, Rasinski K: The Psychology of Survey Response Cambridge, Cambridge University Press 2000

22. Wikman A, Wärneryd B: Measurement errors in survey questions: Explaining response variability. Soc Sci Med 1990, 22:199-212.

23. Torsheim T, Wold B, Samdal O, Haugland S: Test-retest reliability of survey indicators measuring adolescent health and health behaviour. Bergen: Research Centre for Health Promotion, University of Bergen 1997.

24. Boyce W, Torsheim T, Currie C, Zambon A: The family affluence scale as a measure of national wealth. validation of an adele

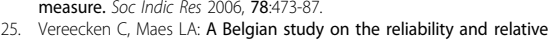
validity of the Health Behaviour in School-aged Children food frequency 作

26. Elgar FJ, Roberts C, Tudor-Smith C, Moore L: Validity of self-reported height and weight and predictors of bias in adolescents. $J$ Adolesc Health 2005, 37:371-5

27. Booth ML, Okely AD, Chey T, Bauman A: The reliability and validity of the physical activity questions in the WHO health behaviour in schoolchildren (HBSC) survey: a population study. Br I Sports Med 2001, 35:263-7.

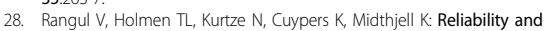
validity of two frequently used self-administered physical activity .

29. Vuori M, Ojala K, Tynjälä J, Villberg J, Välimaa R, Kannas L: The stability of physical activity survey items in the HBSC study Liikunto \& Tiede 2005 , :39-46, In Finnish with English abstract.

. Haugland S, Wold B: Subjective health complaints in adolescenceReliability and validity of survey methods. J Adolesc 2001, 24:611-24. Ojala K, Vuori M, Valimaa R, Vilberg J, Tynjala J, Kannas L: Reasons for exercise inventory in a school survey: contemplations of the inventory's
reliability and structure validity. Likunta \& Tieta 2005, 42(6):30-8, In Finnis with English abstract.
32. Tynjälä J: Sleep habits, perceived sleep quality and tiredness among adolescents: a health behavioural approach. PhD thesis University of Jyväskylä, Department of Health Sciences 1999 .

33. Torsheim T, Wold B, Samdal O: The teacher and classmate support scale: Factor structure, test-retest reliability and validity in samples of 13 - and 15-year-old adolescents. Sch Psychol Int 2000, 21(2):195-212.

34. Shrout PE, Fleiss JL: Intraclass correlations: uses in assessing rater reliability. Psychol Bull 1979, 86(2):420-8

35. Landis JR, Koch GG: The measurement of observer agreement for categorical data. Biometrics 1977, 33:159-74.

36. Prochaska JJ, Sallis JF, Long B: A physical activity screening measure for use with adolescents in primary care. Arch Pediatr Adoles Med 2001, 155:554-9.

37. Treuth MS, Hou N, Young DR, Maynard ML: Validity and reliability of the Fels Physical Activity Questionnaire for children. Med Sci Sports Exerc 2005, 37(3):488-95.

38. Hardy LL, Booth ML, Okely AD: The reliability of the adolescent sedentary activity questionnaire (ASAQ). Prev Med 2007, 45(1):71-4.

39. Brener ND, McMannus T, Galuska DA, Lowry R, Wechsler H: Reliability and validity of self-reported height and weight among high school students. Adolesc Health 2002, 32:281-7.

40. Henriksen $L$, Jackson C: Reliability of children's self-reported cigarette smoking. Addict Behav 1999, 24(2):271-7.

41. Tourangeau R. Remembering what happened: Memory errors and survey reports. The Science of Self-Report: Implications for Research and Practice Mahwah, NJ. Lawrence Enbaum Associatesstone AA, Turkkan JS, Bachrach CA, Jobe JB, Kurtzman HS, Cain VS 2000, 29-47.

Pre-publication history

The pre-publication history for this paper can be accessed here http://www.biomedcentral.com/1471-2288/10/73/prepub

doi:10.1186/1471-2288-

Cite this article as: Liu et al: Test-retest reliability of selected items of

Health Behaviour in School-aged Children (HBSC) survey questions
in Beijing, China. BMC Medical Research Methodology 2010 10:73.
Submit your next manuscript to BioMed Central and take full advantage of:

- Convenient online submission

- Thorough peer review

- No space constraints or color figure charges

- Immediate publication on acceptance

- Inclusion in PubMed, CAS, Scopus and Google Schola

- Research which is freely available for redistribution 


\title{
RELIABILITY AND VALIDITY OF FAMILY AFFLUENCE SCALE (FAS II) AMONG ADOLESCENTS IN BEIJING, CHINA
}

\author{
by
}

Liu, Y., Wang, M., Villberg, J., Torsheim, T., Tynjälä, J., Lv, Y. \& Kannas, L. 2012.

Child Indicators Research 5 (2), 235-251.

Reprinted with kind permission by Springer Science and Business Media 


\section{Reliability and Validity of Family Affluence Scale (FAS II) among Adolescents in Beijing, China}

Yang Liu • Mei Wang • Jari Villberg •

Torbjørn Torsheim • Jorma Tynjälä • Yan Lv •

Lasse Kannas

Accepted: 4 November 2011 /Published online: 25 November 2011

(C) Springer Science+Business Media B.V. 2011

Abstract This study comprises two sub-studies. Study I assessed the test-retest reliability of Family Affluence Scale (FAS II) items among 95 students aged 11 and 15 years old in Beijing. Study II investigated the completion rate of traditional indicators (parents' educational level, perceived family wealth, resident area, and school location) measuring socioeconomic status (SES) compared with FAS II, and examined the internal reliability, external and construct validity of the FAS II items in a population of 5876 schoolchildren aged 11, 13 and 15 years old in Beijing. Our study found that the FAS II items have high completion rates $(>99 \%)$ which are better than other SES indicators. Analyses of reliability showed a moderate internal reliability (Cronbach's alpha $=0.58$ ) and at least substantial test-retest reliability (ICC $>0.75$ ). Moderate external validity of FAS II was found by Spearman rank correlation between FAS II and other SES indicators (parental education level and perceived family wealth) $\left(r_{s}=0.48-0.51, p<0.001\right)$ and ordinal regressions. Graphical log-linear Rasch model (GLLRM) showed that FAS has adequate construct validity (few LD and weak DIF). In conclusion, the FAS II is a reliable and valid SES measure for adolescents in the Beijing area.

Keywords Adolescents · Family affluence scale $\cdot$ Health Behaviour in School-aged Children (HBSC) · Reliability · Socioeconomic inequality · Validity

Y. Liu $(\bowtie) \cdot J$. Villberg $\cdot$ J. Tynjälä $\cdot$ L. Kannas

Research Centre for Health Promotion, Department of Health Sciences, University of Jyväskylä, P.O. Box 35 (Viveca), Jyväskylä 40014, Finland

e-mail: yang.liu@jyu.fi

M. Wang

Mass Research Centre, China Institute of Sport Science, Beijing, China

T. Torsheim

Department of Psychosocial Science, University of Bergen, Bergen, Norway

Y. Lv

Zhejiang Institute of Sports Science, Hangzhou, China 


\section{Introduction}

Social inequalities in health have been an important research topic in both social science and medicine (Dalstra et al. 2006; Marmot 2005). Research on the association of social inequalities and health behaviour also revealed that socio-economic status plays a role in the explanation of the variant prevalence of the health behaviour (Ritterman et al. 2009). Moreover, health inequalities in young children and adults are well-established, but there has been debate about health inequalities in adolescents (West and Sweeting 2004). Inconsistent or non-associations have been found between socioeconomic status (SES) and some health outcomes among adolescents (Goodman 1999; Goodman et al. 2001; Starfield et al. 2002; West 1997; West and Sweeting 2004). Despite the genuine relationships of SES and health outcomes, the results and findings vary according to sample age groups and research methods, one possible reason for this is that various measures of SES were employed and therefore exert different effects on the same health outcome (Currie et al. 2008a), and there might not be sufficient indicators to measure SES of adolescents specifically (Boyce et al. 2006). In the last two decades, the Chinese economy has grown rapidly, resulting in the achievement of improving people's health as well as improving health care. However, these health developments did not automatically follow the economic growth. In actuality, a widening gap in both health status and health care between different social economic statuses was found more often in China ( $\mathrm{Li}$ and Zhu 2006; Liu et al. 1999). Thus, it is crucially important to develop reliable and valid indicators to measuring SES in China in order to get an accurate picture of the evidence-based results of socioinequalities in health.

For an adult population, SES is traditionally assessed by education, occupation and income (Ostrove and Adler 1998). Adolescents, normally, are in the period of spending most of their time studying in school and not legally allowed to work, so they do not have any, or have very little economic resources themselves. Accordingly, adolescents' SES is usually measured by using the information of their parents' SES, such as parents' education and occupation, and household income. However, one should be aware of the difficulties of measuring adolescents' SES when using their parental SES as a proxy. As Currie and her colleagues (2008a) argued conceptually, it is still uncertain whether parents' SES should be used as a proxy. For instance, the adolescents' SES are not always reflecting their parental SES, and even if it does so, which is the most relevant aspect of SES. Furthermore, methodologically there are also difficulties in obtaining accurate information regarding parental SES from adolescents due to the lack of knowledge or unwillingness to reveal the relevant information (Currie et al. 2008a) which results in the low completion or high nonresponse rates when collecting data of adolescents self-reported parental SES (Molcho et al. 2007; Wardle et al. 2002). In addition, it has been reported that there is a higher non-response rate for those adolescents in the low SES group (Wardle et al. 2004). Therefore, when measuring the SES of adolescents, it is crucially important to formulate accurate and effective indicators, which are easy for adolescents to answer and minimize the potential bias in a certain subgroup.

Among the indicators used for measuring adolescents' SES, the Health Behaviour in School-Aged Children (HBSC) Family Affluence Scale (FAS) is a measure which has recently been developed and widely used to address the association of SES and 
health outcomes in both domestically and internationally comparative studies (Currie et al. 2008b; Maes et al. 2006; Pickett et al. 2005; Torsheim et al. 2004a, b; Torsheim et al. 2006). The FAS used in the HBSC survey contains several items which assess the SES of the adolescent based on the material condition of their household, which was originally based on the work of Carstairs and Morris (1991) and Townsend (1987). In the 1997/1998 HBSC survey, three FAS items were employed, which asked about family car ownership, the child's use of their own bedroom, and the number of family holidays, referred to as the first version of FAS (FAS I) (Currie et al. 2004). Later, in the 2001/2002 HBSC survey, an item on number of family computers was added and these four FAS items was named as the second version of FAS (FAS II) (Currie et al. 2004).

The FAS II has been used extensively in the HBSC study in the past decade to examine and describe socioeconomic inequalities in relation to adolescent health outcomes. Compared to the traditional SES indicators, one advantage of the FAS II items is that it can greatly reduce the non-response rate. It has been reported that the FAS II items showed a higher completion rate than items on parental education and occupation (Boudreau and Poulin 2009; Lin 2011; Molcho et al. 2007). Validation studies of the FAS II have been done in many HBSC member countries as well as some non-HBSC member countries, from which the results showed that the FAS II items have a moderate internal reliability (Lin 2011; Molcho et al. 2007; Wardle et al. 2002), a significant association with parental education and occupation (Currie et al. 1997; Molcho et al. 2007), a high agreement rate between adolescents and parents (Andersen et al. 2008), and a satisfactory correlation with the national wealth indicator, Gross Domestic Product (GDP) (Boyce et al. 2006). Although the test-retest reliability of FAS II items has not been examined, the rather higher agreement can be predicted due to the objectivity and stability of the indicators (Currie et al. 2008a). Moreover, Schnohr and her colleagues (2008) have analyzed the differential item functioning (DIF) of FAS II and demonstrated that it can be used as an interval scale and a measure of wealth within a country and between countries.

The FAS II has also recently been used as a SES indicator outside the context of the HBSC study (e.g. West and Sweeting 2004) or in countries which are not a member of the HBSC network (e.g. Cho and Khang 2010). Because every country has its own culture and economic status, the FAS II should be used after examining the applicability and validity in a certain context. However, since the HBSC study is limited at the moment to countries in Europe and North America, few studies assessing the use of the FAS II have been done elsewhere in the world. To the authors' knowledge, there are only two studies which have been done recently to assess the use of FAS II in Asia (Cho and Khang 2010; Lin 2011) and no similar study has been done in the mainland China. The purposes of this study are, therefore, to investigate the completion rate of the traditional indicators measuring SES compared with FAS II, and to examine the reliability and validity of the FAS II items in a Chinese adolescent population.

\section{Methods}

This paper comprises data from two separate studies conducted by the China Institute of Sport Science (CISS) in the Beijing area, both following the HBSC 
research protocol (Currie et al. 2001). Beijing is the capital of China, consisting of 16 districts and 2 counties, with the total population 16.95 million by the end of 2009. Beijing has 653255 students in 1104 primary schools and 508327 students in 634 secondary and upper secondary schools (Beijing Municipal Bureau of Statistics 2010). In 2010, the average annual income in Beijing was 29073 Chinese Yuan (around 4419\$) per person, which is approximately 1.2 times the average annual income for China (3633\$) (Beijing Municipal Bureau of Statistics 2010).

\subsection{Study I. Test-Retest Reliability Study}

The data for test-retest reliability analysis was from the pilot study for the HBSC based behaviour and lifestyle survey for school-aged children in Beijing in October 2008. A sample of 95 male and female students aged 11 or 15 years old participated in a test and retest with a three weeks interval (the demographic characteristics of respondents can be seen in Table 1). Student identity numbers of respondents were utilized to permit matching of test-retest questionnaires. The test-retest reliability of FAS II items was estimated with the single measure of Intraclass Correlation Coefficients (ICC) (Shrout and Fleiss 1979) through case 2 (using a two-way random model with an absolute agreement type), with $95 \%$ confidence interval (CI). The detailed information of Study I is described elsewhere (Liu et al. 2010).

\subsection{Study II. Internal Reliability and Validity Study}

\subsubsection{Study Subjects}

The data for all the other analyses were from the HBSC based behaviour and lifestyle survey for school-aged children in Beijing, which was carried out between November and December 2008 by the China Institute of Sport Science

Table 1 Demographic

characteristics of respondents in Study I and Study II

\begin{tabular}{|c|c|c|c|c|}
\hline & \multicolumn{2}{|c|}{ Sample size } & \multicolumn{2}{|l|}{ Age } \\
\hline & $\mathrm{n}$ & $\%$ & Mean age & $\mathrm{SD}$ \\
\hline \multicolumn{5}{|l|}{ Study I } \\
\hline Total & 95 & 100.0 & I & I \\
\hline Boys & 51 & 53.7 & / & I \\
\hline Girls & 44 & 46.3 & I & I \\
\hline $11 \mathrm{yrs}$ & 44 & 46.3 & 11.70 & 0.35 \\
\hline $15 \mathrm{yrs}$ & 51 & 53.7 & 15.80 & 0.32 \\
\hline \multicolumn{5}{|l|}{ Study II } \\
\hline Total & 5876 & 100.0 & I & I \\
\hline Boys & 2816 & 47.9 & I & I \\
\hline Girls & 3060 & 52.1 & I & I \\
\hline $11 \mathrm{yrs}$ & 1941 & 33.0 & 11.74 & 0.32 \\
\hline $13 \mathrm{yrs}$ & 1930 & 32.8 & 13.73 & 0.34 \\
\hline $15 \mathrm{yrs}$ & 2005 & 34.1 & 15.78 & 0.32 \\
\hline
\end{tabular}


(CISS). The survey sampled from state schools all over the Beijing area. The Chinese school and class systems are not widely known in western countries: sometimes students in the schools, and even in the classes of a school, are arranged according to geographic location, academic achievement etc. Therefore, in order to effectively choose the most representative sample, the survey sampling included three stages and two strata that introduced the detail as follows:

\section{Stage I. Selection of the Sample Districts (Counties)}

The stratified random sample method was used in this stage. The first step of this stage was to stratify all the 16 districts and 2 counties of Beijing area according to geographical location. Two strata, urban area and rural area were stratified in this step. The second step was to stratify both the urban and rural areas in terms of social economic development level. Three strata, good, fair and poor, were used. The last step of this stage was to choose one district (county) randomly from each level in both urban and rural areas.

\section{Stage II. Selection of the Sample Schools}

The stratified random sample method was used in this stage. Firstly, all schools in each sample district (county) were divided into three categories on the basis of school conditions (good, fair and poor). Then, one primary school, one secondary school and one upper secondary school were selected randomly from each category in each sample district (county). In order to ensure the convenience of the survey, both the secondary school and the upper secondary school could be drawn from the same high school which includes both secondary and upper secondary school.

Stage III. Selection of the Sample Classes

The random sampling method or stratified random sampling method is used in this stage.

A total of 5985 schoolchildren were sampled following the above mentioned three stages. Samples were cleaned in line with the cleaning rule of the HBSC survey protocol if their gender or birth date were missing, or their age was beyond the target range (Currie et al. 2001). The final data used for analyses contained a total of 5876 schoolchildren, making a completion rate of $98.2 \%$ (the demographic characteristics of respondents can be seen from Table 1).

\subsubsection{Survey Procedure}

All students in the selected sample class were asked to complete a self-report questionnaire during a normal school class with a teacher and/or researcher administering. The students were first instructed how to fill in the questionnaire. Student's participation in the survey was voluntary and the questionnaire could only be accessed by researcher. Students were also informed that only the researcher will read their answers. The questionnaire used in the Chinese survey was based on the English version of the questionnaire used in the 
Finnish HBSC Survey in 2006. The questionnaire was firstly translated from English to Chinese by two researchers independently and re-translated from Chinese to English by other professional translators to check for any discrepancies. Both studies mentioned here were approved by the ethics committee of CISS and the Research Centre for Health Promotion at the University of Jyväskylä.

\subsubsection{Variables Used in Analysis}

Family Affluence Scale II The FAS II was used as a measure of SES in this study. The items, response categories, codes and analyses strategy of FAS II used in the present study are the following:

- "Does your family own a car, van or truck?" Response categories were: No $(=0)$; Yes, one $(=1)$; Yes, two or more $(=2)$.

- "Do you have your own bedroom for yourself?" Response categories were: No $(=0)$; Yes $(=1)$.

- "During the past 12 months, how many times did you travel away on holiday with your family?" Response categories were: Not at all $(=0)$; Once $(=1)$; Twice $(=2)$; More than twice $(=3)$.

- “How many computers does your family own?" Response categories were: None $(=0)$; One $(=1)$; Two $(=2)$; More than two $(=3)$.

According to Currie and her colleagues (2008a), a composite FAS score was calculated for each respondent based on his or her answers to these four items. Following previous HBSC surveys, the two highest response categories (' 2 ' and ' 3 or more') of the last two items (holidays and computers) were combined. Three groups were categorized in terms of the composite FAS score, in which FAS low $($ score $=0-3)$ indicated low affluence, FAS medium $($ score $=4,5)$ indicated middle affluence, and FAS high $($ score $=6,7)$ indicated high affluence.

Parents' Educational Level Students were asked to choose their father's and mother's highest level of education from 7 alternatives, which are under primary school, primary school, secondary school, upper secondary school, junior college, university (bachelor level), and graduate or more. During the analyses, the parents' education were divided into three categories, low education level (under the primary school, primary school, secondary school), medium education level (upper second school, junior college), and high education level (university, graduate or more), for father and mother respectively.

Perceived Family Wealth Perceived socioeconomic status of the household was enquired by asking the students' own perception of their family financial situation. The responses were very well off financially, quite well off financially, average, not very well off financially and not at all well off financially. The indicator of perceived family wealth was divided into three categories, perceived low family wealth (not very well off, not well off at all), perceived medium family wealth (average), and perceived high family wealth (very well off, quite well off).

Springer 
Urban and Rural According to the sampling procedure, the information of selected schools in the survey can be retrieved, and the respondents were divided into urban and rural according to the school position.

School Location The respondents were categorized into three SES groups, good, fair and poor, according to their school's location in districts with different socioeconomic development (Beijing Municipal Bureau of Statistics 2010).

\subsubsection{Statistical Analyses}

The analyses have been done to address the following aspects of the data according to the purpose of the study:

Item Completion Rate Completion rate was computed for FASII items and total FAS scores as well as the other two SES indicators (parental educational level and perceived family wealth). Chi-square test was used to compare the completion rate of FAS items by gender and by age groups as well as the completion rate among different SES measures.

Reliability The internal consistency of the FAS II items and item-rest coefficient were examined with the Cronbach's alpha coefficient. Spearman correlations were used to assess the association between individual FAS II item, the total FAS scores and FAS category. In order to examine whether the four FAS II items are to reflect a general underlying dimension of affluence, the fit of a one-factor solution was tested with confirmatory factor analysis (CFA) for categorical data using the Robust Weighted Least Square (WLSMV) estimator. The primary fit indices included the Comparative Fit Index (CFI) and the weighted root mean square residual (WRMR), as recommended in recent publications (Finney and DiStefano 2006). A CFI $\geq 0.95$ and WRMR $<1$ indicates a good fit to the data.

Validity Spearman correlations were used to assess the association between the total FAS scores with parental educational level and perceived family wealth. Two ordinal logistic regression models were used for analyzing the association between FAS category and other SES indicators. The unadjusted odds ratio (OR) from model 1 (univariate ordinal regression model) and the adjusted OR from model 2 (included all independent variables) were computed respectively. Construct validity was investigated with the Graphical Log Linear Rasch Model (GLLRM) with Differential Item Function (DIF) and Local Dependence (LD).

All data of the present study were entered by Epidata 3.1 with double entry and validation. The analyses of item completion rate, reliability and validity were done by Predictive Analytics Software (PASW, formerly SPSS), version 18.0 (SPSS, Inc., Chicago, IL, US). The CFA was analyzed by MPlus 6.1 (Muthén and Muthén 2010). The GLLRM was performed by DIGRAM 2.0 (Kreiner 2009). A $P$-value which was lower than 0.05 or $95 \%$ confidence intervals did not overlap was considered significantly different. 


\section{Results}

3.1 Study I

\subsubsection{Test-Retest Reliability}

Table 2 reveals the values of ICC for all respondents by gender and age. Overall, the values of ICC of the FAS II items, FAS score and FAS category ranged from 0.76 to 0.95 , with the lowest value for the item regarding family holidays, and the highest value for the item asking about own bedroom. According to Landis and Koch divisions of agreement (1977), all four items of FAS II as well as the FAS score and category showed at least substantial test-retest reliability. Gender and age differences of the agreement were only found for the item regarding own bedroom.

\subsection{Study II}

\subsubsection{Completion Rate}

In general, all items measuring SES in our study have a completion rate higher than $97 \%$. Out of 5876 pupils, the first and second lowest completion rate were items for parental education (father $97.9 \%$ and mother $98.4 \%$ ) and all the four items of FAS II have the same highest completion rate $(99.7 \%)$, which made the completion rate of the composite FAS scores $99.2 \%$. However, no significant differences were found between the completion rates of the four FAS II items and two parental education items. We also examined the completion rates by gender and by age groups. There is no difference in the completion rates of those SES items by gender except for the question asking about the family holidays $(p=0.042)$ which showed that boys were slightly less likely to report their family holidays than girls. Significant differences of completion rates were found in the three age groups for the item about asking father's education $(p<0.001)$ and mother's education $(p<0.001)$, which indicated that younger children were less likely to report their parental educational level.

\subsubsection{Internal Reliability}

As seen in Table 3, we examined the internal consistency of FAS II items and found a moderate association $(\alpha=0.58)$. The alpha coefficient changed if single item was deleted from the scale. The item-rest coefficients suggested that deleting either family car $(\alpha=0.47)$, or family holidays $(\alpha=0.49)$, or numbers of computer $(0.43)$ items resulted in a drop of internal consistency. On the contrary, if own bedroom was deleted from the FAS II items, the alpha coefficient would improve to 0.60. Higher internal consistencies were observed in older respondents.

The results of the inter-correlations between the FAS II items and the composite FAS score showed moderate associations (more than 0.60) except for the item asking about own bedroom (0.34) (Table 4). Rather poor correlations were found within the FAS II items (from 0.16 to 0.39 ) and the poorest one was found between items asking about own bedroom and family car (0.16), but all items were intercorrelated. 
Table 2 ICC values for FAS II items, FAS score and FAS category by gender and age from Study I ( $N=95)$

\begin{tabular}{|c|c|c|c|c|c|c|c|c|c|c|}
\hline & \multicolumn{2}{|c|}{ All $(N=95)$} & \multicolumn{2}{|c|}{ Boys $(n=51)$} & \multicolumn{2}{|c|}{ Girls $(n=44)$} & \multicolumn{2}{|c|}{11 yrs $(n=44)$} & \multicolumn{2}{|c|}{$15 \operatorname{yrs}(n=51)$} \\
\hline & ICC & $95 \%$ CI & ICC & $95 \%$ CI & ICC & $95 \%$ CI & ICC & $95 \%$ CI & ICC & $95 \%$ CI \\
\hline Family car & 0.88 & $0.83-0.92$ & 0.85 & $0.75-0.91$ & 0.92 & $0.86-0.96$ & 0.81 & $0.68-0.89$ & 0.92 & $0.86-0.95$ \\
\hline Own bedroom & 0.95 & $0.93-0.97$ & 1.00 & I & $0.92^{\mathrm{a}}$ & $0.86-0.96$ & 0.79 & $0.65-0.88$ & $1.00^{\mathrm{b}}$ & 1 \\
\hline Family holidays & 0.76 & $0.66-0.83$ & 0.72 & $0.55-0.83$ & 0.77 & $0.61-0.87$ & 0.78 & $0.64-0.88$ & 0.64 & $0.44-0.79$ \\
\hline No. of computers & 0.77 & $0.67-0.84$ & 0.77 & $0.63-0.86$ & 0.76 & $0.61-0.86$ & 0.73 & $0.56-0.84$ & 0.79 & $0.66-0.87$ \\
\hline FAS score & 0.88 & $0.83-0.92$ & 0.85 & $0.75-0.91$ & 0.92 & $0.84-0.96$ & 0.83 & $0.70-0.90$ & 0.88 & $0.80-0.93$ \\
\hline FAS category & 0.78 & $0.69-0.85$ & 0.73 & $0.57-0.84$ & 0.84 & $0.72-0.91$ & 0.81 & $0.67-0.89$ & 0.68 & $0.50-0.80$ \\
\hline
\end{tabular}

${ }^{a}$ Significant difference between gender groups

${ }^{\mathrm{b}}$ Significant difference between age groups 
Table 3 Cronbach Alpha coefficient for FAS II items by gender and age from Study II $(N=5876)$

\begin{tabular}{lllllll}
\hline \multicolumn{7}{l}{ Cronbach Alpha } \\
\cline { 2 - 7 } & Total & Boys & Girls & 11 yrs & 13 yrs & 15 yrs \\
\hline All four items & 0.58 & 0.59 & 0.57 & 0.56 & 0.56 & 0.62 \\
Delete family car & 0.47 & 0.49 & 0.45 & 0.48 & 0.46 & 0.49 \\
Delete own bedroom & 0.60 & 0.61 & 0.59 & 0.55 & 0.57 & 0.66 \\
Delete family holiday & 0.49 & 0.50 & 0.48 & 0.49 & 0.45 & 0.53 \\
Delete No. Of computers & 0.43 & 0.43 & 0.42 & 0.40 & 0.42 & 0.47 \\
\hline
\end{tabular}

The CFA analyzed by MPlus showed that the one-factor model fitted the data relatively well as indicated by the comparative fit index (CFI) of 0.99 and the weighted root mean square residual of 1.10. The standardised factor loading ranged between 0.49 (number of own bedroom) and 0.69 (number of computers).

\subsubsection{Validity}

The external validity of FAS II was evaluated by examining associations between FAS II and other SES indicators, using Spearman rank correlation $\left(r_{s}\right)$, univariate and multivariate ordinal logistic regression analyses. Moderate associations were found between the composite FAS II scores with father's education $\left(r_{s}=0.49\right.$, $p<0.001)$, mother's education $\left(r_{s}=0.48, p<0.001\right)$, and perceived family wealth $\left(r_{s}=0.51, p<0.001\right)$.

Table 5 shows the distribution of all variables used in this study by FAS category and the unadjusted and adjusted odds ratios (OR's) for all independent variables, with FAS low as the referent group. Students in rural areas were more likely to be located in the low FAS group. The percentage of respondents in high FAS group increased if they reported their father or mother has a higher educational background. Similarly, the percentage of students in high FAS group decreased with their perceived family wealth. Both models revealed the same significant association between FAS II category and all independent variables (Table 5). OR's for gender and age showed that girls and elder students were more likely to report less family affluence, compared to boys and young students. Similar findings were also established for students who lived in rural areas or studied in a school from the district with poor economic status. The multivariate analyses indicated that students

Table 4 Spearman correlations of FAS II items from Study II $(N=5876)$

\begin{tabular}{lcccc}
\hline & Family car & Own bedroom & Family holidays & No. Of computers \\
\hline Own bedroom & $0.16^{* *}$ & & & \\
Family vacations & $0.32^{* *}$ & $0.22^{* *}$ & & \\
No. of computers & $0.39^{* *}$ & $0.20^{* *}$ & $0.36^{* *}$ & $0.69^{* *}$ \\
FAS score & $0.67^{* *}$ & $0.34^{* *}$ & $0.69^{* *}$ & \\
\hline
\end{tabular}

** Correlation is significant at the 0.01 level (2-tailed).

Springer 


\begin{tabular}{|c|c|c|c|c|c|c|c|c|c|c|c|}
\hline & \multirow{2}{*}{$\begin{array}{l}\text { Total } \\
\mathrm{n}\end{array}$} & \multicolumn{2}{|c|}{ FAS (low) } & \multicolumn{2}{|c|}{ FAS (middle) } & \multicolumn{2}{|c|}{ FAS (high) } & \multicolumn{2}{|c|}{ Univariate OLR } & \multicolumn{2}{|c|}{ Multivariate OLR } \\
\hline & & $\mathrm{n}$ & $\%$ & $\mathrm{n}$ & $\%$ & $\mathrm{n}$ & $\%$ & OR & $95 \% \mathrm{CI}$ & OR & $95 \% \mathrm{CI}$ \\
\hline Overall & 5876 & 2421 & 41.2 & 2178 & 37.1 & 1229 & 20.9 & & & & \\
\hline \multicolumn{12}{|l|}{ Gender } \\
\hline Boy & 2816 & 1116 & 39.6 & 1015 & 36.0 & 655 & 23.3 & $0.84 * * *$ & $0.77-0.93$ & $0.82 * *$ & $0.79-0.88$ \\
\hline Girl & 3060 & 1305 & 42.6 & 1163 & 38.0 & 574 & 18.8 & 1.00 & & 1.00 & \\
\hline \multicolumn{12}{|l|}{ Age } \\
\hline 11 & 1941 & 624 & 32.2 & 776 & 40.0 & 525 & 27.0 & $0.48^{* * *}$ & $0.43-0.54$ & $0.54 * * *$ & $0.48-0.62$ \\
\hline 13 & 1930 & 779 & 40.4 & 753 & 39.0 & 373 & 19.3 & $0.70^{* * *}$ & $0.63-0.79$ & $0.67 * * *$ & $0.59-0.77$ \\
\hline 15 & 2005 & 1018 & 50.8 & 649 & 32.4 & 331 & 16.5 & 1.00 & & 1.00 & \\
\hline \multicolumn{12}{|l|}{ Area } \\
\hline Urban & 2833 & 833 & 29.4 & 1136 & 40.1 & 843 & 29.8 & $0.37^{* * *}$ & $0.33-0.40$ & $0.75^{* * *}$ & $0.64-0.84$ \\
\hline Rural & 3043 & 1588 & 52.2 & 1042 & 34.2 & 386 & 12.7 & 1.00 & & 1.00 & \\
\hline \multicolumn{12}{|c|}{ School location } \\
\hline Level 1 & 1943 & 626 & 32.2 & 750 & 38.6 & 555 & 28.6 & $0.39 * * *$ & $0.35-0.44$ & $0.57 * * *$ & $0.49-0.66$ \\
\hline Level 2 & 1956 & 731 & 37.4 & 798 & 40.8 & 409 & 20.9 & $0.53 * * *$ & $0.47-0.60$ & $0.62^{* * *}$ & $0.54-0.72$ \\
\hline Level 3 & 1977 & 1064 & 53.8 & 630 & 31.9 & 265 & 13.4 & 1.00 & & 1.00 & \\
\hline \multicolumn{12}{|c|}{ Father's education } \\
\hline Low & 2017 & 1329 & 65.9 & 539 & 26.7 & 130 & 6.4 & $11.87 * * *$ & $10.29-13.71$ & $3.05 * * *$ & $2.46-3.78$ \\
\hline Middle & 2278 & 845 & 37.1 & 968 & 42.5 & 446 & 19.6 & $3.48^{* * * *}$ & $3.06-3.96$ & $1.66^{* * *}$ & $1.40-1.95$ \\
\hline High & 1456 & 187 & 12.8 & 624 & 42.9 & 637 & 43.8 & 1.00 & & 1.00 & \\
\hline \multicolumn{12}{|c|}{ Mother's education } \\
\hline Low & 2154 & 1384 & 64.3 & 600 & 27.9 & 146 & 6.8 & $11.31^{* * *}$ & $9.80-13.07$ & $2.54 * * *$ & $2.05-3.15$ \\
\hline Middle & 2301 & 818 & 35.5 & 999 & 43.4 & 470 & 20.4 & $3.34 * * *$ & $2.93-3.81$ & $1.55^{* * * *}$ & $1.31-1.83$ \\
\hline
\end{tabular}




\begin{tabular}{|c|c|c|c|c|c|c|c|c|c|c|c|}
\hline & \multirow{2}{*}{$\begin{array}{l}\text { Total } \\
\mathrm{n}\end{array}$} & \multicolumn{2}{|c|}{ FAS (low) } & \multicolumn{2}{|c|}{ FAS (middle) } & \multicolumn{2}{|c|}{ FAS (high) } & \multicolumn{2}{|c|}{ Univariate OLR } & \multicolumn{2}{|c|}{ Multivariate OLR } \\
\hline & & $\mathrm{n}$ & $\%$ & $\mathrm{n}$ & $\%$ & $\mathrm{n}$ & $\%$ & OR & $95 \% \mathrm{CI}$ & OR & $95 \% \mathrm{CI}$ \\
\hline High & 1327 & 177 & 13.3 & 545 & 41.1 & 597 & 45.0 & 1.00 & & 1.00 & \\
\hline \multicolumn{12}{|c|}{ Perceive family wealth } \\
\hline Low & 752 & 605 & 80.5 & 113 & 15.0 & 27 & 3.6 & $21.43^{* * *}$ & $17.44-26.34$ & $10.51^{* * *}$ & $8.44-13.12$ \\
\hline Middle & 2918 & 1444 & 49.5 & 1126 & 38.6 & 325 & 11.1 & $5.02 * * *$ & $4.49-5.62$ & $3.25^{* * *}$ & $2.88-3.67$ \\
\hline High & 2185 & 369 & 16.9 & 930 & 42.6 & 870 & 39.8 & 1.00 & & 1.00 & \\
\hline \multicolumn{12}{|c|}{${ }^{* *}$ overall effect of the variable in the model $p<0.01$} \\
\hline \multicolumn{12}{|c|}{$* * *$ overall effect of the variable in the model $p<0.001$} \\
\hline \multicolumn{12}{|c|}{$* * *$ overall effect of the variable in the model $p<0.001$} \\
\hline
\end{tabular}


whose fathers' educational level was low and middle were respectively around 3 times and 1.7 times more likely to report less affluence than students whose fathers' educational level were high. Similar results were also observed for respondents with low and middle level educational mothers, where the OR's were about 2 and 1.6 respectively. The most prominent finding was that students whose perceived family wealth was low were 10 times more likely to report their family affluence was also low, compared to those who perceived their family wealth as high.

To highlight the construct validity of the indicators, the GLLRM model fitting item responses for our data is shown in Fig. 1. Two coefficients were significant revealing LD (Local Dependence) between items; number of cars-number of computers $\left(\gamma^{\mathrm{p}}=\right.$ $0.15)$ and number of cars-number of holidays $\left(\gamma^{\mathrm{p}}=-0.13\right)$, which meant that there were correlation between the number of cars and number of computers, as well as the number of cars and number of holidays. However, these correlations are weak. The DIF (Differential Item Functioning) was observed between age and three items, 'own bedroom', 'number of holidays', and 'number of computers'. The results indicate that the chances of having own bedroom $\left(\gamma^{\mathrm{p}}=0.41\right)$ and number of computers $\left(\gamma^{\mathrm{p}}=0.16\right)$ increased with age, whereas the number of holidays $\left(\gamma^{\mathrm{p}}=-0.28\right)$ decreased with age The DIF was not found between gender and FAS II items.

\section{Discussion and Conclusion}

To obtain the accurate finding of social inequalities in health, it is critical to employ reliable and valid indicators which measure socioeconomic status. Although FAS II, widely used in HBSC network countries in Europe and North America, was proved to be an easily answered and a useful indicator of child material affluence, it should be examined whether FAS II is applicable before it is used in countries other than the current HBSC countries. As the first study to assess the usability of FAS II in a Chinese context, in general, we found that of the FAS II items have high completion rates, a moderate internal reliability, at least substantial test-retest reliability,

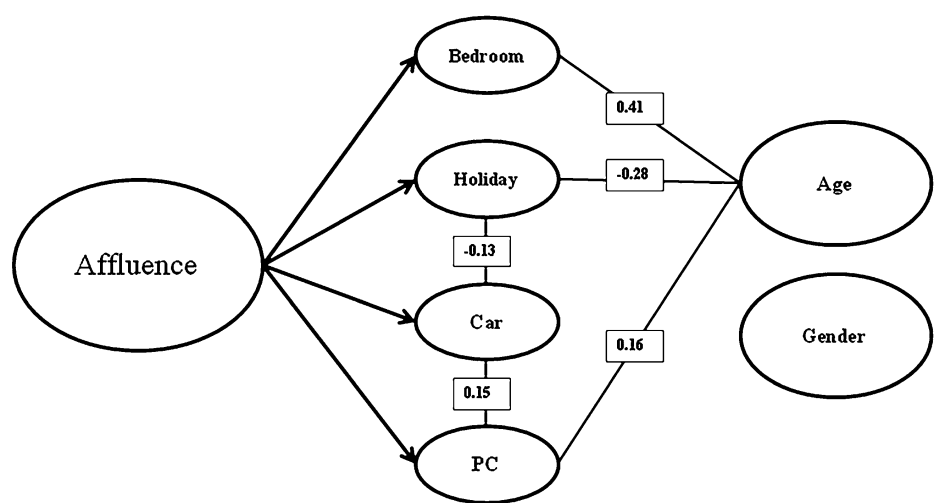

Fig. 1 The generalization of FAS II to GLLRM (number in boxes are partial gamma coefficients, $\gamma^{\mathrm{p}}$, indicate a significant correlation beyond the latent trait) from Study II $(N=5876)$ 
moderate associations with other SES indicators, only few LD and weak DIF. This indicates that the FAS II is a useful and valid SES measure for adolescents in the Beijing area.

Previous studies demonstrated the difficulties of obtaining information on parental education, occupation, and income, which are usually used as SES markers in adolescent studies and it has been reported in many studies that there is a high non-response rate for those questions (Cho and Khang 2010; Currie et al. 1997; Lin 2011; Molcho et al. 2007; Wardle et al. 2002). In contrast, it has been highlighted in previous studies that the FAS II items have much higher completion rates than other traditional SES indicators (Cho and Khang 2010; Lin 2011; Molcho et al. 2007; Wardle et al. 2002). In our study, therefore, it is not surprising that a higher proportion of the respondents answered the four FAS II items (99.7\%) than items on parents' highest educational level $(97.9 \%$ for father and $98.4 \%$ for mother). However, the completion rates for parental education questions in our study were much higher than previous studies, although they revealed the lowest completion rates among all SES indicators analyzed in the present study. One possible reason might be that students were instructed to answer all the questions in sequence at the beginning of the survey which generated very low non-response rate for all survey questions. Consequently, no significant difference was found between the completion rates of the four FAS II items and the other SES indicators. When comparing the completion rates of the SES measures across gender and age, we found that there was no gender difference, which is similar to the results reported by Molcho and his colleagues (2007) except for the question asking about the family holidays $(p=0.042)$. Age differences were found for items asking about parental education $(p<0.001)$, indicating that younger children were less likely to report their parental educational level, again, similar results were also found by Molcho et al. (2007) and Lin (2011). We also found age differences of two FAS II items, family car $(p=0.009)$ and own bedroom $(p=0.001)$, which are not consistent with other studies (Lin 2011; Molcho et al. 2007).

Concerning the reliability of FAS II, in our study, the internal correlations between the FAS II items were low but all items were intercorrelated $\left(r_{s}=0.16-0.39\right.$, $p<0.001)$ and the Cronbach's alpha was moderate $(0.58)$, which is in line with previous studies (Cho and Khang 2010; Lin 2011; Molcho et al. 2007; Schnohr et al. 2008). These results were understandable due to the limited range of the alternatives and each item describing a different aspect of family affluence (Wardle et al. 2002). Similar to earlier studies (Boudreau and Poulin 2009; Cho and Khang 2010; Lin 2011), we found that the item on own bedroom had weaker correlations with the other FAS II items and the composite FAS score. In addition, the item-rest coefficients in our study concluded that if the item on own bedroom was deleted from the FAS scale, the alpha coefficient would improve whereas deleting any other of the three items would reduce the internal consistency. Those findings suggested that further examination should be considered to choose the most sensitive items in specific settings since country and culture difference may affect the relative contribution of the four FAS II items to the composite FAS II (Schnohr et al. 2008). One extra item which may be considered and tested in the future is that to ask the amount of weekly pocket money received by the student, which the child will reliably know the answer to, and is related to family affluence to some extent. 
The significant association between FAS II and other traditional SES measures, such as parental education and/or parental occupation (Lin 2011; Molcho et al. 2007; Richter et al. 2009) were found in previous studies, although the strength of associations varied in different countries. The rather low strength of association found in the present study was accounted for by the fact that FAS and parental education and occupation examine different aspects of socioeconomic status per se, and therefore may not overlap (Molcho et al. 2007; Wardle et al. 2002). Our study revealed moderate associations between FAS and other SES indicators. The strength of associations in the present study $\left(r_{s}=0.48-0.51, p<0.001\right)$ were higher than previous findings possibly indicating that the FAS II was related to other SES measures more closely in a Chinese context than others. We also assessed the external validity of FAS II by ordinal logistic regression and the results were in line with the correlation analyses which confirmed the significant association between FAS II and other SES indicators. For instance, the students who perceived their family wealth as low were 10 times more likely to be in the low FAS category than those who perceived their family wealth as high, suggesting that the FAS II was more likely measuring the similar dimension of SES as perceived family wealth. This finding presented the possibility of adding the item on perceived family wealth as one item of the FAS scale for the development of the family affluences measure in the future, in line with Schnohr and her colleagues' (2008), who have purposed that the FAS II can be used as a measure of wealth within a country and between countries, plus the item on perceived family wealth was included. However, we should notice that perceived family wealth is a subjective concept, and therefore it may not be comparable to measures of the FAS II which are more concrete in nature. Furthermore, the results of GLLRM model presented in this study indicated that the FAS II items are nearly locally independent and have very weak DIF, which can be considered as satisfied construct validity according to Rosenbaum (1989).

The strength of the present study is that we examined the test-retest reliability of FAS II, which has not been done and reported in earlier studies. As expected by Currie and her colleagues (2008a), the results revealed that all four FAS II items and the composite FAS score and category had at least substantial test-retest reliability due to the objectivity of the measures. Moreover, it is the first study to examine the cross cultural application of the FAS II comprehensively using a large sample of Chinese adolescents. At the same time, the current study still has several limitations. First of all, the data were only sampled from the Beijing area due to the aims of the survey and the limited resources. As a country with huge socioeconomic difference such as China, it may raise the bias to determine the usability of the FAS II if the sample cannot represent the whole nation. Another major limitation is that the data were retrieved from self-report questionnaires from the adolescents and there is a lack of objective measures of respondents' family wealth, for instance, investigating the financial circumstances of the parents. In order to examine and develop the FAS II as a SES measure in China, more research with other objective measures should be encouraged in more and wilder locations.

In conclusion, as the first study on the reliability and validity of the HBSC FAS II in an adolescent population in Beijing, the present study showed that the FAS II had a high completion rate, better than other SES indicators. Moderate internal reliability and at least substantial test-retest reliability, as well as adequate external and 
construct validity, were found for the FAS II. In conclusion, the FAS II is a useful and valid SES measure for adolescents in the Beijing area.

Acknowledgements The authors would like to thank Ms. Lanmin Xiao from Beijing Experimenta School of Xicheng District and Ms. Jing Tian from Beijing Academy of Educational Sciences for helping to organize and conduct the field work. The authors also would like to thank Mr. Michael Ormshaw for checking the language of the manuscript. As a part of joint research project, this study was supported by the China Institute of Sport Science (CISS) and the Research Centre for Health Promotion at the Department of Health Science in the University of Jyväskylä. The survey and data collection were funded by the CISS. During this research, the first author was supported by grants from the Finnish Foundation for Alcohol Studies and the University of Jyväskylä.

\section{References}

Andersen, A., Krølner, R., Currie, C., Dallago, L., Due, P., Richter, M., Orkényi, A., \& Holstein, B. E. (2008). High agreement on family affluence between children's and parents' reports: international study of 11-year-old children. Journal of Epidemiology and Community Health, 62, 1092-1094.

Beijing Municipal Bureau of Statistics. (2010). Beijing statistical year book. Beijing: China Statistical Publishing House.

Boudreau, B., \& Poulin, C. (2009). An examination of the validity of the Family Affluence Scale II (FAS II) in a general adolescent population of Canada. Social Indicators Research, 94, 29-42.

Boyce, W., Torsheim, T., Currie, C., \& Zambon, A. (2006). The Family Affluence Scale as a measure of national wealth: validation of an adolescent self-report measure. Social Indicators Research, 78, 473-487.

Carstairs, V., \& Morris, R. (1991). Deprivation and health in Scotland. Aberdeen: Aberdeen University Press.

Cho, H. J., \& Khang, Y. H. (2010). Family Affluence Scale, other socioeconomic position indicators, and self-rated health among South Korean adolescents: findings from the Korea Youth Risk Behavior Web-based Survey (KYRBWS). Journal of Public Health, 18(2), 169-178.

Currie, C., Elton, R. A., Todd, J., \& Platt, S. (1997). Indicators of socioeconomic status for adolescents: the WHO Health Behaviour in School-aged Children Survey. Health Education Research, 12(3), 385-397.

Currie, C., Samdal, O., Boyce, W., \& Smith, R. (Eds.). (2001). Health Behaviour in Schoolaged Children: a WHO Cross-National Study (HBSC), Research Protocol for the 2001/2002 Survey. Edinburgh: Child and Adolescent Health Research Unit (CAHRU), University of Edinburgh.

Currie, C., Roberts, C., Morgan, A., Smith, R., Settertobulte, W., Samdal, O., \& Barnekow, V. (Eds.) (2004). Young people's health in context. Copenhagen: WHO Regional Office for Europe.

Currie, C., Molcho, M., Boyce, W., Holstein, B. E., Torsheim, T., \& Richter, M. (2008a). Researching health inequalities in adolescents: the development of the Health Behaviour in School-Aged Children (HBSC) Family Affluence Scale. Social Science \& Medicine, 66, 1429-1436.

Currie, C., Nic Gabhainn, S., Godeau, E., Roberts, C., Smith, R., Currie, D., Pickett, W., Morgan, A., \& Barnekow, V. (Eds.). (2008b). Inequalities in young people's health: HBSC international report from the 2005/2006 survey. Copenhagen: WHO Regional Office for Europe.

Dalstra, J., Kunst, A., \& Mackenbach, J. (2006). A comparative appraisal of the relationship of education, income and housing tenure with less than good health among the elderly in Europe. Social Science \& Medicine, 62, 2046-2060.

Goodman, E. (1999). The role of socioeconomic status gradients in explaining differences in US adolescents' health. American Journal of Public Health, 89, 1522-1528.

Goodman, E., Adler, N. E., Kawachi, I., Frazier, A. L., Huang, B., \& Colditz, G. A. (2001). Adolescents perceptions of social status: development and evaluation of a new indicator. Pediatrics, 108(2), 1-8.

Finney, S. J., \& DiStefano, C. (2006). Non-normal and categorical data in structural equation modeling. In G. R. Hancock \& R. O. Mueller (Eds.), Structural equation modeling: A second course. Greenwich: Information Age Publishing.

Kreiner, S. (2009). A guided tour through DIGRAM 2.0: Analysis of contingency tables by chain graph models. Copenhagen: Department of Biostatistics, University of Copenhagen.

Landis, J. R., \& Koch, G. G. (1977). The measurement of observer agreement for categorical data Biometrics, 33, 159-174.

\section{Springer}


Li, H., \& Zhu, Y. (2006). Income, income inequality, and health: evidence from China. Journal of Comparative Economics, 34, 668-693.

Lin, Y. C. (2011). Assessing the use of the Family Affluence Scale as socioeconomic indicators for researching health inequalities in Taiwan adolescents. Social Indicators Research, 102, 463-475.

Liu, Y., Hsiao, W., \& Eggleston, K. (1999). Equity in health and health care: the Chinese experience. Social Science \& Medicine, 49, 1349-1356.

Liu, Y., Wang, M., Tynjälä, J., Lv, Y., Villberg, J., Zhang, Z., \& Kannas, L. (2010). Test-retest reliability of selected items of Health Behaviour in School-aged Children (HBSC) survey questionnaire in Beijing, China. BMC Medical Research Methodology, 10, 73 .

Maes, L., Vereecken, C., Vanobbergen, J., \& Honkala, S. (2006). Tooth brushing and social characteristics of families in 32 countries. International Dental Journal, 56, 159-167.

Marmot, M. (2005). Social determinants of health inequalities. The Lancet, 365, 1099-1104.

Molcho, M., Nic Gabhainn, S., \& Kelleher, C. (2007). Assessing the use of the Family Affluence Scale (FAS) among Irish schoolchildren. Irish Medical Journal, 100(8), 37-39.

Muthén, L. K., \& Muthén, B. O. (2010). Mplus User's Guide (6th ed.). Los Angeles: Muthén \& Muthén. Ostrove, J. M., \& Adler, N. E. (1998). Socioeconomic status and health. Current Opinion in Psychiatry, $11,649-653$.

Pickett, W., Molcho, M., Simpson, K., Janssen, I., Kuntsche, E., Mazur, J., Harel, Y., \& Boyce, W. (2005). Cross-national studyof injury and social determinants in adolescents. Injury Prevention, 11, 213-218.

Richter, M., Erhart, M., Vereecken, C. A., Zambon, A., Boyce, W., \& Gabhainn, S. N. (2009). The role of behavioural factors in explaining socio-economic differences in adolescent health: A multilevel study in 33 countries. Social Science \& Medicine, 69(3), 396-403.

Ritterman, M. L., Fernald, L. C., Ozer, E. J., Adler, N. E., Gutierrez, J. P., \& Syme, S. L. (2009). Objective and subjective social class gradients for substance use among Mexican adolescents. Social Science \& Medicine, 68, 1843-1851.

Rosenbaum, P. R. (1989). Criterion-related construct validity. Psychometrika, 54, 625-633.

Schnohr, C., Kreiner, S., Due, P., Currie, C., Boyce, W., \& Diderichsen, F. (2008). Differential item functioning of a family affluence scale: validation study on data from HBSC 2001/02. Social Indicators Research, 89(1), 79-95.

Shrout, P. E., \& Fleiss, J. L. (1979). Intraclass correlations: use in assessing rater reliability. Psychological Bulletin, 86(2), 420-428.

Starfield, B., Riley, A. W., Witt, W. P., \& Robertson, J. (2002). Social class gradients in health during adolescence. Journal of Epidemiology and Community Health, 56, 354-361.

Torsheim, T., Currie, C., Boyce, W., Kalnins, I., Overpeck, M., \& Haughland, S. (2004a). Material deprivation and self-rated health: a multilevel study of adolescents from 22 European and North American countries. Social Science \& Medicine, 59(1), 1-12.

Torsheim, T., Valimaa, R., \& Danielson, M. (2004b). Health and well-being. In C. Currie, C. Roberts, A. Morgan, R. Smith, W. Settertobulte, O. Samdal, \& V. Barnekow Rasmussen (Eds.), Young people's health in context: Health Policy for children and adolescents no. 4 (pp. 55-62). Copenhagen: WHO Regional Office for Europe.

Torsheim, T., Currie, C., Boyce, W., \& Samdal, O. (2006). Country material distribution and adolescents' perceived health: multilevel study of adolescents in 27 countries. Journal of Epidemiology and Community Health, 60, 156-161.

Townsend, P. (1987). Deprivation. Journal of Social Policy, 16(2), 147-164.

Wardle, J., Robb, K., \& Johnson, F. (2002). Assessing socioeconomic status in adolescents: the validity of a home affluence scale. Journal of Epidemiology and Community Health, 56, 595-599.

Wardle, J., Robb, K. A., Johnson, F., Griffith, J., Brunner, E., Power, C., \& Martin, T. (2004). Socioeconomic variation in attitudes to eating and weight in female adolescents. Health Psychology, 23(3), 275-282.

West, P. (1997). Health inequalities in the early years: is there equalization in youth? Social Science and Medicine, 44, 833-858.

West, P., \& Sweeting, H. (2004). Evidence on equalization in health in youth from the West of Scotland. Social Science \& Medicine, 59(1), 13-27. 


\title{
SOCIOECENOMIC INEQUALITIES IN ALCOHOL USE OF ADOLESCENTS: THE DIFFERENCE BETWEEN CHINA AND FINLAND
}

\author{
by \\ Liu, Y., Wang, M., Tynjälä, J., Villberg, J., Lv, Y. \& Kannas, L. 2013. \\ International Journal of Public Health 58 (2), 177-185.
}

Reprinted with kind permission by Springer Science and Business Media 


\title{
Socioeconomic inequalities in alcohol use of adolescents: the differences between China and Finland
}

\author{
Yang Liu $\cdot$ Mei Wang $\cdot$ Jorma Tynjälä $\cdot$ Jari Villberg • \\ Yan Lv $\cdot$ Lasse Kannas
}

Received: 20 December 2011/Revised: 15 October 2012/Accepted: 19 November 2012/Published online: 28 November 2012 (C) Swiss School of Public Health 2012

\section{Abstract}

Objectives Findings from previous studies on socioeconomic inequalities in alcohol use among adolescents are not consistent. This study examined and compared the relationship between socioeconomic status and alcohol use of adolescents in China and Finland.

Methods The sample included 2,005 Chinese and 1,685 Finnish 15-year-old school children. The associations between Family Affluence Scale (FAS) and alcohol use were examined separately in two countries through binary logistic regression.

Results Chinese adolescents from the high FAS group were more likely to report experiencing monthly alcohol use $(\mathrm{OR}=2.13)$ and early onset of alcohol use $(\mathrm{OR}=$ $1.55)$ (girls), and early onset of drunkenness $(\mathrm{OR}=1.81$ ) (boys). However, no statistically significant difference was found in three FAS groups for all four measures of alcohol use among Finnish adolescents.

Conclusions This study indicated clear differences of socioeconomic inequalities in alcohol use between Chinese and Finnish adolescents. It suggested that interventions on adolescent alcohol use in China should be targeted on those who are from the high affluent family whereas in Finland

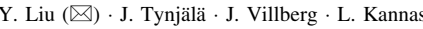
Research Centre for Health Promotion, Department of Health Sciences, University of Jyväskylä, Jyväskylä, Finland e-mail: yang.liu@jyu.fi

M. Wang

Mass Sport Research Centre, China Institute of Sport Science, Beijing, China

Y. Lv

Zhejiang Institute of Sport Science, Hangzhou, China
}

efforts should be made to curb adolescents drinking across the socioeconomic spectrum.

Keywords Adolescent - Alcohol use - Drunkenness . Family Affluence Scale $\cdot$ Socioeconomic inequalities

\section{Introduction}

The evidence of health inequalities has been accumulated from both social and medical research (Marmot 2005; Dalstra et al. 2006). Most of the existing studies concerning socioeconomic inequalities, however, focused on adults only. Studies concerning socioeconomic inequalities' impact on adolescent health have rarely been conducted until recently (DiLiberti 2000; Marmot 2005) and there has been a debate about health inequalities in adolescence (Currie et al. 2008a, b; Marmot 2005; West and Sweeting 2004). It has been well documented that low socioeconomic status has been consistently related to health-risk behaviours among adults (Pampel et al. 2010), while the associations among adolescents were not as uniform as those in adulthood (Hanson and Chen 2007). For instance, research on associations between socioeconomic status (SES) and adolescents' risky health behaviours, such as smoking (Holstein et al. 2004; Richter and Leppin 2007) and alcohol use (Elgar et al. 2005; Richter et al. 2006), have revealed inconsistent results.

Alcohol use of young people has been a global concern and it has been reported that the use of alcohol in adolescence can be a major problem resulting in significan disability and premature death (WHO 2004) and other risks in health, such as injury (Zeigler et al. 2005), alcohol misuse (Gruber et al. 1996), and mental problems (Wechsler et al. 1998). Alcohol is closely intertwined in 
almost every aspect of Chinese culture. Since the 1980s, the alcohol consumption in China has increased rapidly (WHO 2004), and alcohol use among Chinese youth need to be addressed. A survey conducted in three middle schools in Beijing showed that life-time drinking rates were 48 and $37 \%$ for boy and girl junior students, respectively, and 73 and $56 \%$ for boy and girl senior students. $12 \%$ admitted that they had experienced drunkenness in the last year (Xing et al. 2002). Drinking behaviour of school-aged children is also very common in Finland. A recent survey based on the Finnish family competence study showed that at 15 years of age, $83 \%$ of girls and $79 \%$ of boys had used alcohol, and $18 \%$ of boys and $14 \%$ of girls had been drunk more than once a month (Seljamo et al. 2006). In Finland, adolescents' drinking habits are drunkenness-orientated. The most recent results from the Health Behaviour in School-aged Children (HBSC) study revealed that although the weekly alcoho consumption of Finnish school-aged children is below the HBSC average and even the lowest in the age group of 15 years, the proportion of 15 -year-olds who reported first getting drunk at age 13 or younger are much higher than the HBSC average (Currie et al. 2008b).

Several studies have investigated the presence of socioeconomic inequalities of alcohol use (Kunst et al. 1996; Bloomfield et al. 2006), and the clear and persistent social gradient in alcohol-related mortality among adults have been documented by former studies (Mäekelä et al. 2003; Harrison and Gardiner 1999). Most studies, however, focused only on the adult population and there is not enough research targeting alcohol use of adolescents. In addition, the evidence based on previous studies about the relationship between socioeconomic status and adolescent drinking behaviour is inconsistent and even contradictory. For instance, some studies revealed a higher adolescent drinking behaviour among lower socioeconomic groups (Melotti et al. 2011), while others reported that there is no, only limited or even inverse social gradients in adolescent alcohol use (Vereecken et al. 2003; Richter et al. 2006). The findings from the latest HBSC reported that the association between alcohol use and family affluence differed within the participation countries (Currie et al. 2008b).

The Family Affluence Scale (FAS) provides a unique approach to examine and describe socioeconomic inequalities in relation to adolescent health behaviours and has been used extensively in the HBSC study in the past decade (Currie et al. 2008a). Previous studies proved that FAS is a useful and valid SES measure for adolescents in HBSC member countries (Molcho et al. 2007; Wardle et al. 2002) as well as in Beijing, China (Liu et al. 2012). Through using FAS as the same and comparable SES measure, we can examine and compare the relationship between SES and health behaviours of adolescents in different countries. In order to examine whether the FAS is more sensitive in a certain context, and therefore make further developments, more comparative studies, using the FAS as a SES indicator should be conduct in different contexts, such as developing and developed countries, or countries with small and big socioeconomic inequalities.

Findings from previous innumerous studies on socioeconomic inequalities and alcohol use of adolescents are not consistent and universal, which highlights the need for further study. Furthermore, previous studies were mainly conducted in most of developed countries from North America and Europe (Richter et al. 2006). However, such comparative studies have seldom been done between Europe and Asian countries. More research in a wide range of countries/regions needs to be carried out to understand the whole picture of this issue.

Regardless of the differences of culture, economic, geographical and political context, both China and Finland intend to decrease the prevalence of alcohol use of adolescents and health inequalities. Meanwhile, Finland represents a developed European country with a good social welfare system and rather less socioeconomic inequalities, and China represents a developing Asian country with an underdeveloped social welfare system and rather high socioeconomic inequalities. Research on socioeconomic inequalities in alcohol use between these two countries can provide the evidence on whether socioeconomic status influences adolescents' alcohol use independently of their cultural and political contexts, the findings of which can also be valuable for policy makers and health practitioners in all countries concerned. Therefore, the present study aims to address the following research questions: (1) what are the associations between the socioeconomic status and adolescents' alcohol use in China and Finland? (2) does socioeconomic inequalities in alcohol use of adolescent differ in these two countries?

\section{Methods}

The data used in the present study comprised data from both China and Finland. The Chinese data used in this study were from the results of the Health Behaviour and Lifestyle Survey for School-aged Children in Beijing, which was conducted in December 2008 using the same questionnaire items and research protocol as the HBSC survey used in Finland (Currie et al. 2001). The Chinese survey was conducted by the Chinese Institute of Sport Science (CISS) and it was approved by the ethics committee of the CISS. The Finnish data were obtained from the HBSC study 2005/2006, a World Health Organization collaborative cross-national survey, of which the Finnish survey was conducted between March and May 2006. The 
Finnish HBSC study was conducted by the Research Centre for Health Promotion at the Department of Health Sciences of the University of Jyväskylä and it was approved by the Finnish National Board of Education (FNBE) and the Trade Union of Education in Finland.

\section{Sample}

The target population for the HBSC study is young people who are attending school aged 11, 13 and 15 years old Approximately 1,500 respondents in each of the three age groups were recruited in the survey countries. The present study only analysed data gathered from adolescents aged 15 years old, due to the rather low prevalence of alcohol use among 11 and 13-year-old adolescents in both China and Finland.

The Chinese survey sample was from state schools in Beijing area using multi-stage strata and random cluster method. The detailed information of sampling procedure in China can be found elsewhere (Liu et al. 2012). The response rates for schools and students in China were 90.0 and $100 \%$. The Finnish survey sample was gathered from state schools through Finland via cluster sampling with probability proportional to size (PPS) of schools method. The response rates for schools and students in Finland were 95.2 and $84.5 \%$ The sample size used and data analysed in this study consisted of 1,685 Finnish and 2,005 Chinese 15-year-old adolescents. Overall, the sample size, gender proportion and the mean age are very similar in the two countries (Table 1).

Instrument and variables

The HBSC survey was based on completion of a self-report questionnaire during a normal school class with a teacher and/or researcher administering and supervising. The

Table 1 Demographic characteristics of respondents and distribution of family affluence scale groups by country (China 2008 and Finland 2006)

\begin{tabular}{|c|c|c|c|c|c|}
\hline \multicolumn{3}{|c|}{ China } & \multicolumn{3}{|c|}{ Finland } \\
\hline$n$ & $\%$ & Age & $n$ & $\%$ & Age \\
\hline & & Mean SD & & & Mean SD \\
\hline
\end{tabular}

\begin{tabular}{|c|c|c|c|c|c|c|c|c|}
\hline \multicolumn{9}{|l|}{ Gender } \\
\hline Boys & 892 & 44.5 & 15.82 & 0.32 & 790 & 46.9 & 15.79 & 0.33 \\
\hline Girls & 1,113 & 55.5 & 15.75 & 0.33 & 895 & 53.1 & 15.76 & 0.31 \\
\hline \multicolumn{9}{|l|}{ FAS } \\
\hline Low (0-3) & 1,025 & 51.1 & 15.80 & 0.32 & 262 & 15.6 & 15.82 & 0.31 \\
\hline $\begin{array}{l}\text { Middle } \\
(4,5)\end{array}$ & 649 & 32.4 & 15.76 & 0.32 & 792 & 47.0 & 15.78 & 0.32 \\
\hline High $(6,7)$ & 331 & 16.5 & 15.75 & 0.33 & 631 & 37.4 & 15.76 & 0.32 \\
\hline Total & 2,005 & 100.0 & 15.78 & 0.32 & 1,685 & 100.0 & 15.78 & 0.32 \\
\hline
\end{tabular}

students were instructed on how to fill in the questionnaire and also informed that only the researcher will read their answers. Student's participation in the survey was voluntary. The questionnaire used in the Chinese survey was based on the English version of the questionnaire used in the Finnish HBSC Survey in 2006. The questionnaire was firstly translated from English to Chinese by two researchers independently and re-translated from Chinese to English by other professional translators to check for any discrepancies. The survey questionnaire has satisfactory test-retest reliability for the students in Beijing (Liu et al. 2010). All survey procedures followed the guidelines provided by the HBSC research protocol (Currie et al. 2001).

\section{Measurements of alcohol use}

Alcohol consumption Alcohol consumption was assessed by the item 'At present, how often do you have the following alcohol drinks? Also include those times when you only drink a very small amount'. For each type of beverage: beer, wine, strong liquor, mild alcohol drinks, and any other drink that contains alcohol, the possible answers were: 'daily', 'at least once a week', 'at least once a month', 'less', and 'never'. Monthly alcohol use was defined as drinking any alcohol at least once a month.

Onset of alcohol use The initiation of alcohol use was examined by asking the questions 'At what age did you drink alcohol (more than a small amount) for the first time?' The answers were never, 11 years old or less, 12 years old, 13 years old, 14 years old, 15 years old or older. Early onset of alcohol use was defined as first drinking alcohol at age 13 or younger.

Drunkenness Frequency of drunkenness was assessed by asking the respondents whether they had ever had so much alcohol that they had been really drunk. The response alternatives were: 'never', 'yes, once', 'yes, 2-3 times', 'yes, 4-10 times' and 'yes, more than 10 times'. Frequent drunkenness was defined as $2-3$ or more times.

Onset of drunkenness The initiation of drunkenness was examined by asking the questions 'At what age you were drunk for the first time?' The answers were: never, 11 years old or less, 12 years old, 13 years old, 14 years old, 15 years old or older. Early onset of drunkenness was defined as those respondents who reported they were first drunk at age 13 or younger.

Measurements of socioeconomic status

Family Affluence Scale The FAS has been used to examine and explain socioeconomic inequalities in the 
HBSC study for more than 10 years (Currie et al. 2008a), and it has also been shown as a reliable and valid SES measure for adolescents in China (Liu et al. 2012). The scale comprises four items: does your family own a car $(0,1,2$ or more)? Do you have your own room $(0,1)$ ? How many times over the last year did you travel away somewhere on holiday with your family $(0,1,2,3$ or more)? And, how many computers does your family own $(0,1,2,3$ or more)? A composite FAS score was calculated by summing the responses of these four items ranging from 0 to 7 . The composite scores were subsequently recoded into high $(6,7)$, middle $(4,5)$, and low $(0-3)$ family affluence. The distribution of three FAS groups in two countries can be seen from Table 1 .

Statistical analyses

Descriptive statistics were used to show the characteristics of the study sample and the distribution of FAS groups Differences in percentage of alcohol use in the two countries and in the three FAS groups were compared by Pearson's Chi-square test. The above-mentioned analyses were conducted separately for boys and girls and for China and Finland, and a $p$ value of $<0.05$ was considered statistically significant. In addition, binary logistic regression models were used to investigate the relationships between all variables of alcohol use and FAS category for the two countries. In each model, the associations were evaluated by gender and countries and the low FAS group served as the reference category with odds ratios and $95 \%$ confidence intervals (CIs) being computed for the middle and high FAS groups for comparison. In logistic regression analyses, $95 \%$ CIs do not overlap were considered statistically significant. All analyses were done by Analytics Software (PASW, formerly SPSS), version 18.0 (SPSS, Inc., Chicago, IL, USA).

\section{Results}

The prevalence of alcohol use of Chinese and Finnish adolescents is shown in Tables 2 and 3. In general, the percentage of monthly alcohol use of Chinese 15-year-old students is $18.7 \%$ while the percentage of Finnish adolescents is approximately double $(39.2 \%)$, of which both Finnish boys and girls reported monthly alcohol use more often than Chinese boys and girls. For the onset of alcohol use, nearly one-fourth of Chinese adolescents and more than one-third of Finnish adolescents reported that they had drunk alcohol at age 13 or younger. No significant difference was found for early onset of alcohol use between Chinese and Finnish boys. However, the results indicate that more Finnish girls (32.3\%) have early onset of alcohol use than Chinese girls $(20.5 \%)$. Similar to the results for alcohol consumption, Finnish respondents reported nearly five times greater frequent drunkenness and three times greater early onset of drunkenness than Chinese respondents. The gender difference is clear among Chinese adolescents; more boys experienced monthly alcohol use, frequent drunkenness and early onset of alcohol use and drunkenness than girls. However, this situation cannot be found among Finnish students. Especially for the proportion of early onset of alcohol use, for which Finnish girls are even slightly higher than boys. In general, the results reveal that, except for early onset of alcohol use among boys, the four measures of alcohol use have statistically significant differences among Chinese and Finnish adolescents.

The percentages for alcohol use in different FAS groups are shown in Table 4. Although a significant difference was only found for monthly alcohol use of Chinese girls and for early onset of drunkenness of Chinese boys, we can see that, compared with the adolescents in the low FAS group, both Chinese boys and girls who are in the middle and high FAS groups reported higher likelihood of alcohol use. Apart from the result of monthly alcohol use among Chinese boys, alcohol use difference in line with the change of the FAS groups among Chinese students. Irregular results were observed among Finnish students: the highes proportion of alcohol use can be found in the middle FAS group for girls and in the low or middle groups for boys. The differing reports of alcohol use for Finnish adolescents in the three FAS groups do not reach the level of statistical significance.

We employed binary logistic regression models to investigate the associations between all variables of alcohol use and FAS category by gender for China and Finland and odds ratios with $95 \%$ CIs are shown in Table 5 . When the low FAS group was used as a reference group, Chinese girl respondents who belong to the high FAS group are more likely to report experiencing monthly alcohol use $(\mathrm{OR}=2.13)$ and early onset of alcohol use $(\mathrm{OR}=1.55)$ Meanwhile, the Chinese boys categorised in the high FAS group have a risk of reporting more early onset of drunkenness $(\mathrm{OR}=1.92)$ compared with those who are in the low FAS group. However, the regression analyses revealed that, compared with the Chinese results, no significantly lower or higher odds ratios were found among Finnish boys and girls in both the middle and the high FAS groups.

\section{Discussion}

Overall, this study found that monthly alcohol use and early onset of alcohol use is rather common in both China and Finland. Compared to China, many more Finnish 
Table 2 Prevalence of alcohol use by gender and country (China 2008 and Finland 2006)

\begin{tabular}{|c|c|c|c|c|c|c|c|c|c|c|c|c|}
\hline & \multicolumn{4}{|l|}{ Total } & \multicolumn{4}{|c|}{ China } & \multicolumn{4}{|c|}{ Finland } \\
\hline & \multicolumn{2}{|l|}{ China } & \multicolumn{2}{|c|}{ Finland } & \multicolumn{2}{|l|}{ Boys } & \multicolumn{2}{|l|}{ Girls } & \multicolumn{2}{|c|}{ Boys } & \multicolumn{2}{|c|}{ Girls } \\
\hline & $n$ & $\%$ & $n$ & $\%$ & $n$ & $\%$ & $n$ & $\%$ & $n$ & $\%$ & $n$ & $\%$ \\
\hline \multicolumn{13}{|l|}{ Alcohol consumption } \\
\hline Never & 617 & 30.8 & 364 & 21.6 & 241 & 30.1 & 376 & 33.8 & 183 & 23.2 & 181 & 20.2 \\
\hline Less than once a month & 1,015 & 50.6 & 662 & 39.3 & 414 & 46.4 & 601 & 54.0 & 290 & 36.7 & 372 & 41.6 \\
\hline At least once a month & 204 & 10.2 & 499 & 29.6 & 119 & 13.3 & 85 & 7.6 & 226 & 28.6 & 273 & 30.5 \\
\hline At least once a week & 139 & 6.9 & 155 & 9.2 & 94 & 10.5 & 45 & 4.0 & 88 & 11.1 & 67 & 7.5 \\
\hline Daily & 30 & 1.5 & 5 & 0.3 & 24 & 2.7 & 6 & 0.6 & 3 & 0.4 & 2 & 0.2 \\
\hline Total & 2,005 & 100 & 1,685 & 100 & 892 & 100 & 1,113 & 100 & 790 & 100 & 895 & 100 \\
\hline \multicolumn{13}{|l|}{ Drunkenness } \\
\hline Never & 1,569 & 78.3 & 761 & 45.1 & 639 & 71.6 & 930 & 83.7 & 348 & 44.0 & 413 & 46.1 \\
\hline Once & 230 & 11.5 & 161 & 9.6 & 114 & 12.8 & 116 & 10.4 & 75 & 9.5 & 86 & 9.6 \\
\hline $2-3$ times & 160 & 8.0 & 281 & 16.7 & 105 & 11.8 & 55 & 4.9 & 123 & 15.6 & 158 & 17.7 \\
\hline 4-10 times & 25 & 1.2 & 213 & 12.6 & 19 & 2.1 & 6 & 0.5 & 99 & 12.5 & 114 & 12.7 \\
\hline More than 10 times & 21 & 1.0 & 269 & 16.0 & 15 & 1.7 & 6 & 0.5 & 145 & 18.4 & 124 & 13.9 \\
\hline Total & 2,005 & 100 & 1,685 & 100 & 892 & 100 & 1,113 & 100 & 790 & 100 & 895 & 100 \\
\hline \multicolumn{13}{|l|}{ Onset of alcohol use } \\
\hline Never & 1,128 & 56.3 & 525 & 31.2 & 423 & 47.4 & 705 & 63.4 & 259 & 32.8 & 266 & 29.7 \\
\hline 11 or less & 266 & 13.3 & 84 & 5.0 & 133 & 14.9 & 133 & 11.9 & 48 & 6.1 & 36 & 4.0 \\
\hline 12 & 114 & 5.7 & 132 & 7.8 & 64 & 7.2 & 50 & 4.5 & 67 & 8.5 & 65 & 7.3 \\
\hline 13 & 112 & 5.6 & 312 & 18.5 & 68 & 7.6 & 44 & 4.0 & 127 & 16.1 & 185 & 20.7 \\
\hline 14 & 139 & 6.9 & 363 & 21.5 & 63 & 7.1 & 76 & 6.8 & 160 & 20.3 & 203 & 22.7 \\
\hline 15 or older & 246 & 12.3 & 269 & 16.0 & 141 & 15.8 & 105 & 9.4 & 129 & 16.4 & 140 & 15.7 \\
\hline Total & 2,005 & 100 & 1,685 & 100 & 892 & 100 & 1,113 & 100 & 790 & 100 & 895 & 100 \\
\hline \multicolumn{13}{|l|}{ Onset of drunkenness } \\
\hline Never & 1,579 & 78.8 & 687 & 40.7 & 642 & 72.0 & 937 & 84.1 & 314 & 39.9 & 373 & 41.6 \\
\hline 11 or less & 76 & 3.8 & 40 & 2.4 & 44 & 4.9 & 32 & 2.9 & 24 & 3.0 & 16 & 1.8 \\
\hline 12 & 34 & 1.7 & 78 & 4.6 & 22 & 2.5 & 12 & 1.1 & 42 & 5.3 & 36 & 4.0 \\
\hline 13 & 43 & 2.1 & 254 & 15.1 & 25 & 2.8 & 18 & 1.6 & 113 & 14.3 & 141 & 15.8 \\
\hline 14 & 79 & 3.9 & 325 & 19.3 & 50 & 5.6 & 29 & 2.6 & 139 & 17.6 & 186 & 20.8 \\
\hline 15 or older & 194 & 9.7 & 301 & 17.9 & 109 & 12.2 & 85 & 7.7 & 158 & 19.9 & 143 & 16.0 \\
\hline Total & 2,005 & 100 & 1,685 & 100 & 892 & 100 & 1,113 & 100 & 790 & 100 & 895 & 100 \\
\hline
\end{tabular}

Table 3 Drinking behaviour by gender and country (China 2008 and Finland 2006)

\begin{tabular}{|c|c|c|c|c|c|c|c|c|c|c|c|c|c|c|c|}
\hline & \multicolumn{5}{|c|}{ Total } & \multicolumn{5}{|c|}{ Boys } & \multicolumn{5}{|c|}{ Girls } \\
\hline & \multicolumn{2}{|c|}{ China } & \multicolumn{2}{|c|}{ Finland } & \multirow[t]{2}{*}{$p$} & \multicolumn{2}{|c|}{ China } & \multicolumn{2}{|c|}{ Finland } & \multirow[t]{2}{*}{$p$} & \multicolumn{2}{|c|}{ China } & \multicolumn{2}{|c|}{ Finland } & \multirow[t]{2}{*}{$p$} \\
\hline & $n$ & $\%$ & $n$ & $\%$ & & $n$ & $\%$ & $n$ & $\%$ & & $n$ & $\%$ & $n$ & $\%$ & \\
\hline Monthly alcohol use & 373 & 18.7 & 659 & 39.2 & $* * *$ & 237 & 26.6 & 317 & 40.2 & $* * *$ & 136 & 12.2 & 342 & 38.2 & $* * *$ \\
\hline Early onset of alcohol use & 492 & 24.7 & 528 & 32.0 & $* * *$ & 265 & 29.9 & 242 & 31.7 & $\mathrm{~ns}$ & 227 & 20.5 & 286 & 32.3 & **** \\
\hline Frequent drunkenness & 206 & 10.3 & 763 & 45.4 & $* * *$ & 139 & 15.7 & 367 & 46.6 & $* * *$ & 67 & 6.1 & 396 & 44.3 & $* * *$ \\
\hline Early onset of drunkenness & 153 & 7.7 & 372 & 22.6 & $* * *$ & 91 & 10.4 & 179 & 22.5 & $* * *$ & 62 & 5.6 & 193 & 21.8 & $* * *$ \\
\hline
\end{tabular}


Table 4 Percentages of alcohol use in different Family Affluent Scale groups by gender and country (China 2008 and Finland 2006)

\begin{tabular}{|c|c|c|c|c|c|c|c|c|c|c|c|c|c|c|c|c|}
\hline & \multicolumn{8}{|c|}{ China } & \multicolumn{8}{|c|}{ Finland } \\
\hline & \multicolumn{4}{|l|}{ Boys } & \multicolumn{4}{|l|}{ Girls } & \multicolumn{4}{|l|}{ Boys } & \multicolumn{4}{|l|}{ Girls } \\
\hline & Low & Middle & High & $\bar{p}$ & Low & Middle & High & $p$ & Low & Middle & High & $\bar{p}$ & Low & Middle & High & $\bar{p}$ \\
\hline Monthly alcohol use & 23.3 & 29.8 & 29.1 & $\mathrm{~ns}$ & 9.7 & 13.7 & 18.7 & ** & 33.3 & 42.9 & 41.3 & $\mathrm{~ns}$ & 38.1 & 40.0 & 36.6 & $\mathrm{~ns}$ \\
\hline Early onset of alcohol use & 28.3 & 29.2 & 35.3 & $\mathrm{~ns}$ & 18.0 & 22.6 & 25.3 & ns & 36.1 & 30.5 & 32.1 & $\mathrm{~ns}$ & 30.6 & 35.1 & 30.0 & $\mathrm{~ns}$ \\
\hline Frequent drunkenness & 14.5 & 14.8 & 19.3 & $\mathrm{~ns}$ & 5.7 & 5.9 & 7.8 & ns & 44.4 & 49.4 & 44.4 & $\mathrm{~ns}$ & 42.5 & 48.3 & 39.9 & ns \\
\hline Early onset of drunkenness & 8.9 & 9.0 & 15.9 & * & 5.1 & 6.2 & 6.5 & ns & 25.2 & 25.2 & 21.4 & $\mathrm{~ns}$ & 21.6 & 24.2 & 19.2 & $\mathrm{~ns}$ \\
\hline
\end{tabular}

$n s$ no significant difference

$* p<0.05, * * p<0.01$

schoolchildren have experienced frequent drunkenness and early onset of drunkenness. Gender differences in alcoho use were hardly found among Finnish respondents, whereas a greater proportion of alcohol use of Chinese youth was contributed by boys. Very interestingly, SES gradients of alcohol use were found among Chinese adolescents, yet those gradients were absent among Finnish adolescents. In addition, some significant effects of family affluence on drinking and drunkenness were observed among Chinese schoolchildren but not among Finnish schoolchildren when analysed by gender.

The findings of the current study regarding socioeconomic inequalities in alcohol use of Chinese adolescents are in line with previous studies conducted in developing countries. For instance, a recent study in Mexico indicated that more risk factors for current drinking were found in the middle or highest tercile of household expenditures compared with the lowest (Ritterman et al. 2009). This pattern was also revealed in a Brazilian study of adults, in which a higher SES was associated with higher rates of alcohol use (Almeida-Filho et al. 2005). These differences support the diffusion of innovation theory that economic factor, as a determinant of adoption, would increase the diffusion process of drinking behaviour (Ferrence 2001). Therefore, those adolescents from well-off families are more likely to adopt the new behaviour (innovation), such as drinking alcoholic beverages or being drunk. However, the patterns of SES and drunkenness of Finnish adolescents reported in the present study are not consistent with Richter and his colleague's study (2006) which was based on the results of the Finnish HBSC survey 2001/2002. This may be caused by the different SES measures used in these two studies, as well as the data derived from different surveys.

The factors affecting the alcohol use in adolescents are complex and there are several plausible interpretations for the differences of socioeconomic inequalities in alcohol use between Chinese and Finnish adolescents which were found by the present study. The first interpretation is that

Table 5 The associations between alcohol use and family affluence scale by gender and country, odds ratios with $95 \%$ confidence intervals (China 2008 and Finland 2006)

\begin{tabular}{|c|c|c|c|c|c|c|c|c|}
\hline & \multicolumn{4}{|c|}{ China } & \multicolumn{4}{|c|}{ Finland } \\
\hline & \multicolumn{2}{|c|}{ Middle } & \multicolumn{2}{|l|}{ High } & \multicolumn{2}{|c|}{ Middle } & \multicolumn{2}{|l|}{ High } \\
\hline & OR & $95 \% \mathrm{CI}$ & OR & $95 \% \mathrm{CI}$ & OR & $95 \% \mathrm{CI}$ & OR & $95 \% \mathrm{CI}$ \\
\hline \multicolumn{9}{|l|}{ Boys } \\
\hline Monthly alcohol use & 1.39 & $0.99-1.95$ & 1.35 & $0.91-2.01$ & 1.51 & $0.96-2.37$ & 1.41 & $0.89-2.23$ \\
\hline Early onset of alcohol use & 1.05 & $0.75-1.46$ & 1.38 & $0.95-2.01$ & 0.78 & $0.49-1.23$ & 0.84 & $0.53-1.33$ \\
\hline Frequent drunkenness & 1.03 & $0.67-1.57$ & 1.42 & $0.89-2.25$ & 1.22 & $0.79-1.89$ & 1.00 & $0.64-1.56$ \\
\hline Early onset of drunkenness & 1.01 & $0.60-1.70$ & 1.92 & $1.13-3.28$ & 0.99 & $0.61-1.65$ & 0.81 & $0.48-1.36$ \\
\hline \multicolumn{9}{|l|}{ Girls } \\
\hline Monthly alcohol use & 1.47 & $0.98-2.20$ & 2.13 & $1.31-3.47$ & 1.09 & $0.71-1.66$ & 0.94 & $0.61-1.46$ \\
\hline Early onset of alcohol use & 1.33 & $0.96-1.84$ & 1.55 & $1.02-2.35$ & 1.22 & $0.78-1.92$ & 0.97 & $0.61-1.55$ \\
\hline Frequent drunkenness & 1.03 & $0.59-1.80$ & 1.39 & $0.70-2.76$ & 1.27 & $0.83-1.92$ & 0.90 & $0.58-1.39$ \\
\hline Early onset of drunkenness & 1.23 & $0.70-2.16$ & 1.30 & $0.62-2.72$ & 1.16 & $0.70-1.91$ & 0.86 & $0.50-1.46$ \\
\hline
\end{tabular}

The reference group in logistic regression was adolescents in low FAS group

Significant difference were shown in bold values 
the adolescents' availability of resources to consume alcohol differs in China and Finland. Compared to the adult population, the accessibility of money for drinking is more limited for adolescents (Kunstche et al. 2004). For instance, a relationship between the amount of pocket money and drunkenness was found among Finnish adolescents (Lintonen et al. 2000; Kouvonen and Lintonen 2002). There is a huge difference in national income and income inequality between China and Finland. China is a country with a rather high income inequality, and adolescents who are part of an affluent family usually have more pocket money and hence they have more possibility to buy alcohol than those in less affluent families. However, with regard to Finnish adolescents, although the amount of pocket money of young people in different FAS groups may still vary, even those who live in rather less affluent families might have enough money to buy alcoholic drinks due to the lower income inequality. Similarly, Elgar and his colleagues (2005) examined the effects of income inequality on drinking behaviour among 11, 13 and 15 -year-old students in 34 industrialized countries and found that income inequality was associated with drinking and drunkenness among younger adolescents but not among older adolescents.

Another possible explanation for the differences could be due to the different drinking culture between eastern and western countries, and when one considers whether adolescents may drink alcoholic beverages alone, or with their family or peers. Traditional Chinese cultural norms encourage social drinking (Cochrane et al. 2003), especially with family/relatives and friends. When Chinese students were asked where they usually did their drinking, the answer was that the majority of them reported it to be at festivals when families get together (Newman 2002). It is understandable that Chinese adolescents in well-off families could have more opportunities to experience drinking at home than their Finnish counterparts, and therefore, drinking in a family situation may play a less important role in affecting alcohol use of adolescents in Finland.

In addition to the above-mentioned two explanations, the sensibility of the FAS, as the indicator of socioeconomic inequality in two countries, should be taken into account for the reasons of the absence of differences in Finland. Although the FAS has been shown as a reliable and valid SES measure in many HBSC member countrie (Currie et al. 2008a), as well as in Beijing, China (Liu et al. 2012), it still needs to be considered whether the socioeconomic inequalities can be detected in a country such as Finland, which has a developed social welfare system and rather small socioeconomic inequalities. The three categories of the FAS may not disclose the socioeconomic difference in the exact same way in Finland as in China.

To our knowledge, this study is the first to examine and compare the socioeconomic inequalities in drinking behaviour and drunkenness of adolescents among an European country and an Asian country. Research on socioeconomic inequalities in alcohol use between countries rather than within one country can provide evidence that socioeconomic status may not influence alcohol use independently of cultural and political contexts, and the differences presented in this study may reflect how different aspects of SES can influence one certain health behaviour in different countries. On the one hand, from theoretical point of view, findings from this study can broaden our knowledge in understanding socioeconomic inequalities of alcohol use in adolescence. On the other hand, from the practical point of view, the evidence-based findings from this study can provide valuable information for policy makers and health practitioners. When planning and implementing interventions/programmes aiming alcohol use of adolescent, the target in China should be the reduction of alcohol use of adolescents from more affluent families, and in Finland should be the reduction of the rather high rates of drinking and drunkenness of adolescence in both genders and all socioeconomic status. In addition, this study suggested that the policy of increasing minimum alcohol price may play a greater role in reducing adolescent alcohol use in Finland than it would in China, due to the fact that the Chinese students who often drink are more likely to be from a more affluent family.

There are several methodological limitations in the present study. First of all, the data were only sampled from the Beijing area due to the aims of the survey and the limited resources. In a country with huge diversity such as China, the results should be interpreted with caution since the sample cannot represent the whole nation. Second, we employed FAS as the only SES measure in this study. In spite the fact that FAS has a very high response rate in the present study and it has been proved to be a reliable and valid measure of family wealth between cultures (Brown et al. 2008) and countries (Schnohr et al. 2008), it is still a reflection of family material affluence which may not be absolutely consistent with the available resources that schoolchildren can use to buy alcohol. Moreover, selfreporting on alcohol use could also raise bias due to the social desirability and the crude/dichotomous classifications of drinking behaviour and drunkenness. Such classifications may lose a certain amount of information which leads to report errors. Besides the methodological limitations, alcohol use is influenced by a variety of factors including marketing, cost, income, national programme and policies, culture, social norms and so on (SimonsMortona et al. 2009) while these factors were not measured and evaluated in this study.

In conclusion, the present study indicated clear socioeconomic inequalities in alcohol use between Chinese and Finnish adolescents. Chinese 15-year-old students from 
more affluent families are more likely to experience alcohol use and drunkenness than those from lower affluent families. In comparison, there is no significant association between family affluence and any measure of alcohol use in Finnish adolescents. Considering the culture and income differences between China and Finland, specific policies and interventions for different target groups should be encouraged and designed for reducing the prevalence of alcohol use of adolescents. Further studies on factors influencing alcohol use of adolescents, such as legislations government policies, should be conducted to understand young people's alcohol use comprehensively.

Acknowledgments The authors would like to thank Ms. Lanmi Xiao for helping to organise the school survey in Beijing and Mr. Zhouyang Zhang for helping to conduct the field work. The authors also would like to thank Mr. Michael Ormshaw for checking the language of the manuscript. As a part of joint research project, this study was supported by the China Institute of Sport Science (CISS) and the Res funded by the Ministry of Social Affairs and Health of Finland. The first author was supported by grants from the Finnish Foundation for Alcohol Studies and the Juho Vainio Foundation.

Conflict of interest The authors declare that there is no known conflict of interest with regards to this research.

\section{References}

Almeida-Filho N, Lessa I, Magalhaes L, Araujo MJ, Aquino E, Jame SA, Kawachi I (2005) Social inequality and alcohol consumption-abuse in Bahia, Brazil: interactions of gender, ethnicity an social class. Soc Psych Psych Epid 40:214-222

Bloomfield K, Grittner U, Kramer S, Gmel G (2006) Social inequalities in alcohol consumption and alcohol related problems in the study countries of the EU concerted action 'gender, culture and alcohol problems: a multi-national study'. Alcohol Alcohol 41(Suppl):i26-i36

Brown RA, Adler NE, Worthman CM, Copeland WE, Costello EJ, Angoled A (2008) Cultural and community determinants of subjective social status among Cherokee and White youth. Ethn Health 13(4):289-303

Cochrane J, Chen H, Conigrave KM, Hao W (2003) Alcohol use in China. Alcohol Alcohol 38(6):537-542

Currie C, Molcho M, Boyce W, Holstein B, Torsheim T, Richter M (2008a) Researching health inequalities in adolescents: the development of the Health Behaviour in School-aged Children (HBSC) family affluence scale. Soc Sci Med 66:1429-1436

Currie C, Nic Gabhainn S, Godeau E et al (2008b) Inequalities in young people's health: HBSC international report from the 2005/2006 survey. WHO Regional Office for Europe, Copenhagen

Currie C, Samdal O, Boyce W, Smith R (2001) Health behaviour i school-aged children: a WHO cross-national study (HBSC), Research protocol for the 2001/2002 survey. Child and Adolescent Health Research Unit (CAHRU), University of Edinburgh, Edinburgh
Dalstra J, Kunst A, Mackenbach J (2006) A comparative appraisal of the relationship of education, income and housing tenure with less than good health among the elderly in Europe. Soc Sci Med 62:2046-2060

DiLiberti JH (2000) The relationship between social stratification and ill-cause mortality among children in the United States. Pediatrics 105(1):1968-1992

Elgar FJ, Roberts C, Parry-Langdon N, Boyce W (2005) Income inequality and alcohol use: a multilevel analysis of drinking and drunkenness in adolescents in 34 countries. Eur J Public Health 15(3):245-250

Ferrence R (2001) Diffusion theory and drug use. Addition 96:165-173 Gruber E, DeClemente RJ, Anderson MM, Lodico M (1996) Early drinking onset and its association with alcohol use and problem drinking onset and its association with alcohol use and

Hanson MD, Chen E (2007) Socioeconomic status and health behaviours in adolescence: a review of literature. J Behav Med 30:263-285

Harrison L, Gardiner E (1999) Do the rich really die young? Alcoholrelated mortality and social class in Great Britain, 1988-94. Addiction 94:1871-1880

Holstein B, Parry-Langdon N, Zambon A, Currie C, Robert C et al (2004) Socioeconomic inequalities and health. In: Currie C, Robert C, Morgan A (eds) Young people's health in context. Health policy for children and adolescents no. 4. WHO regional office, Copenhagen, pp 165-172

Kouvonen A, Lintonen T (2002) Adolescent part-time work and heavy drinking in Finland. Addiction 97:311-318

Kunst A, Cavelaars A, Groenhof F et al. (1996) Socioeconomic inequalities in morbidity and mortality in Europe: a comparative study, vol 1: Main Report. Department of Public Health, Erasmus University, Rotterdam

Kunstche E, Rehm J, Gmel G (2004) Characteristics of binge drinkers in Europe. Soc Sci Med 59:113-127

Lintonen T, Rimpelä M, Vikat A, Rimpelä A (2000) The effect of societal changes on drunkenness trends in early adolescence. Health Educ Res 15:261-269

Liu Y, Wang M, Tynjälä J, Lv Y, Villberg J, Zhang Z, Kannas L (2010) Test-retest reliability of selected items of Health Behaviour in School-aged Children (HBSC) survey. BMC Med Res Methodol 10:73

Liu Y, Wang M, Villberg J, Torsheim T, Tynjälä J, Lv Y, Kannas L (2012) Reliability and validity of family affluence scale (FAS II) among adolescents in Beijing, China. Child Indic Res 5(2):235-251

Mäekelä P, Keskimäki I, Koskinen S (2003) What underlies the high alcohol related mortality of the disadvantaged: high morbidity or poor survival? J Epidemiol Commun H 57:981-986

Marmot M (2005) Social determinants of health inequalities. Lancet 365:1099-1104

Melotti R, Heron J, Hickman M, Macleod J, Araya R, Lewis G (2011) Adolescent alcohol and tobacco use and early socioeconomic position: the ALSPAS birth cohort. Pediatrics 127:e948-e955. doi:10.1542/peds.2009-3450

Molcho M, Nic Gabhainn S, Kelleher C (2007) Assessing the use of the family affluence scale (FAS) among Irish schoolchildren. Irish Med J 100(8):37-39

Newman I (2002) Cultural aspects of drink alcohol controls in China. Global Alcohol Policy Alliance 1:18-21

Pampel FC, Krueger PM, Denney JT (2010) Socioeconomic disparities in health behaviours. Annu Rev Sociol 36:349-370

Richter M, Leppin A (2007) Trends in socio-economic differences in tobacco smoking among German schoolchildren, 1994-2002. Eur J Public Health 17:565-571

Richter M, Leppin A, Gabhainn N (2006) The relationship between parental socio-economic status and episodes of drunkenness 
among adolescents: findings from a cross-national survey. BMC Public Health 6:289

Ritterman ML, Fernald LC, Ozer EJ, Adler NE, Gutierrez JP, Syme SL (2009) Objective and subjective social class gradients for SL (2009) Objective and subjective social class gradients for
substance use among Mexican adolescents. Soc Sci Med substance use

Schnohr CW, Kreiner S, Due P et al (2008) Differential item functioning of a family affluence scale: validation study on data from HBSC 2001/02. Soc Indic Res 89(1):79-95

Seljamo S, Aromaa M, Koivusilta L, Rautava P, Sourander A, Helenius H, Sillanpaa M (2006) Alcohol use in families: 15-year prospective follow-up study. Addiction 101:984-992

Simons-Mortona BG, Pickett W, Boyce W, ter Bogt TFM, Volleberghd W (2009) Cross-national comparison of adolescent drinking and cannabis use in the United States, Canada, and the drinking and cannabis use in the United States,
Netherlands. Int J Drug Policy 21(1):64-69

Vereecken CA, Maes L, Bacquer D (2003) The influence of parenta occupation and the pupils' education level on lifestyle behaviours among adolescents in Belgium. J Adolesc Healt 34:330-338
Wardle J, Robb K, Johnson F (2002) Assessing SES in adolescents: the validity of a home affluence scale. J Epidemiol Commun $\mathrm{H}$ 56:595-599

Wechsler H, Dowdall GW, Maenner G, Gledhill-Hoyt J, Lee H (1998) Changes in binge drinking and related problems among American college students between 1993 and 1997. Results of the Harvard School of Public Health College Alcohol Study. J Am Coll Health 47:57-68

West P, Sweeting H (2004) Evidence on equalization in health in youth from the west of Scotland. Soc Sci Med 59(1):13-27

WHO (2004) Global Status Report on Alcohol 2004. Department of Mental Health and Substance Abuse, Geneva

Xing Y, Ji C, Yang X, Ji H, Yang L, Wang Y, Zhang L (2002) The prevalence of addictive behaviors among middle school students in Shijingshan, Beijing. Chin J Sch Dr 16:684-686

Zeigler DW, Wang C, Yoast RA (2005) The neurocognitive effects of alcohol on adolescents and college students. Prev Med 40:23-32 
Liu, Y., Wang, M., Tynjälä, J., Villberg, J., Lv, Y. \& Kannas, L. 2013.

Submitted for publication 
Socioeconomic differences in smoking of adolescents: a comparison between China and Finland

Yang Liu, Mei Wang², Jorma Tynjälä¹, Jari Villberg1, Yan Lv³, Lasse Kannas ${ }^{1}$

${ }^{1}$ Research Centre for Health Promotion, Department of Health Sciences, University of Jyväskylä, 40014, Jyväskylä, Finland

${ }^{2}$ Mass Sport Research Centre, China Institute of Sport Science, Beijing, China

${ }^{3}$ Zhejiang Institute of Sport Science, Hangzhou, China

*Corresponding author

Email addresses:

YL: yang.liu@jyu.fi

MW: may_wang@homtail.com

JT: jorma.tynjala@jyu.fi

JV: jari.villberg@jyu.fi

YL: lvyandatou@msn.com

LK: lasse.kannas@jyu.fi 


\section{ABSTRACT}

\section{Background}

Findings from previous studies on socioeconomic differences in adolescent smoking are not consistent. The purpose of this study is to examine and compare the relationship between socioeconomic status and adolescent smoking in China and Finland.

\section{Methods}

The sample included 2005 Chinese and 1685 Finnish 15-year-old schoolchildren. The associations between socioeconomic status (SES) measures, including Family Affluence Scale (FAS) and perceived family wealth (PFW), and smoking, including ever smoked, present status of smoking and onset of smoking were examined separately in two countries through binary logistic regression.

\section{Results}

Chinese adolescents from the low (OR $=1.84,95 \% \mathrm{CI}$ : 1.24-2.74) and the middle (OR $=0.43,95 \%$ CI: 0.20-0.92) FAS group are less likely to be a daily smoker. On the contrary, Finnish students from the low $(\mathrm{OR}=1.84,95 \% \mathrm{CI}: 1.24-2.74)$ and the middle $(\mathrm{OR}=1.51,95 \% \mathrm{CI}: 1.13-2.03)$ FAS group are more likely to report being a daily smoker. Significant differences were also found for early onset of smoking in both countries if PFW was served as SES indicator.

\section{Conclusions}

This study indicated clear differences of socioeconomic inequalities in smoking between Chinese and Finnish adolescents. Specific policies and interventions for different target group should be encouraged and designed for reducing the prevalence of alcohol use of adolescents. The study also suggested that different patterns of socioeconomic inequalities in smoking can be found if different indicators of SES and smoking were chosen for analysing.

Keywords: Adolescent, Smoking, Family Affluence Scale, Socioeconomic inequalities 


\section{Background}

Smoking and exposure to tobacco smoke can cause a considerable number of diseases [1] and the World Health Organization (WHO) has identified smoking as the single most preventable cause of death in the world today [2]. In spite of the fact that most regular smokers are adults, the issue of smoking among adolescents is always a public health concern since adolescence is a period when many people initiate smoking, and therefore start the lifestyle of a regular smoker [3].

Socioeconomic factors play an important role in smoking. Numerous studies regarding socioeconomic inequalities and smoking have been done in adult populations and the results indicated that people from lower Socioeconomic Status (SES) groups, defined by income, education, occupation or other SES measures, were more likely to smoke [3-5].

In contrast to studies on adult populations, the literature addressing the socioeconomic inequalities in smoking among adolescents is limited. Most previous studies on health inequalities among adolescents suggest that lower socioeconomic position relates to poorer health and health behaviour, regardless of the measure of SES [6-8]. However, with regard to smoking, the limited literature revealed inconsistent and even contradictionary results. Several studies showed that there is an association between smoking and SES during adolescence $[9,10]$, while other research indicated that there was no relationship between SES and adolescents' smoking $[11,12]$. Moreover, a study analysing trends in socioeconomic differences and smoking among German schoolchildren, found that family affluence only had a weak effect on regular smoking [13]. This variety of results can be found particularly from the Health Behaviour in School-aged Children (HBSC) survey, an international survey in more than 40 countries, in which the results suggested that the relationship between family affluence scale and early initiation of smoking and weekly smoking varies among countries [14].

The prevalence of adolescents' smoking has increased dramatically in China. It was reported that 80,000 Chinese young people become new smoker everyday [15]. Although at present, Finland has reached the stage at which the overall prevalence of smoking is decreasing [16], the smoking rates of adolescents are still not optimistic. The past two Finnish HBSC surveys (2006 and 2010) indicated that one fifth of 15 year-old Finnish schoolchildren reported smoking weekly [14,17]. However, the pattern of socioeconomic inequalities in smoking among Chinese and Finnish young people has not yet been confirmed.

Reducing SES inequalities in smoking and the consequence of smoking is one of the major priorities of public health. Thus, preventing the development of socioeconomic inequalities in smoking among adolescents is crucially important, and therefore it is urgent to design and implement effective prevention programs for the most vulnerable target group based on the comprehensive understanding of the current situation of adolescents' smoking. Research on social inequalities in smoking between countries can provide the evidence on whether SES influences adolescents' smoking independently of their cultural and political contexts, the findings of which can also be valuable for policy makers and health practitioners in all countries con- 
cerned. In terms of the above-mentioned facts, the aims of this study, therefore, are to examine whether socioeconomic inequalities in smoking exist in Chinese and Finnish schoolchildren, by utilizing different measures of SES and smoking behaviour, and therefore to compare the disparities or similarities in the two countries.

\section{Methods}

This paper reports data collected from China and Finland, for which the same HBSC research protocol [18] were used in both countries. The Finnish data were obtained from the HBSC study 2005/2006, conducted between March and May 2006. The Finnish HBSC study was carried out by the Research Centre for Health Promotion at the Department of Health Sciences of the University of Jyväskylä and it was approved by the Finnish National Board of Education and the Trade Union of Education in Finland. The Chinese data were from the Health Behaviour and Lifestyle Survey for School-aged Children in Beijing, which was conducted in December 2008. The Chinese survey was carried out by the China Institute of Sport Science (CISS) and it was approved by the ethics committee of the CISS.

Sample

The Chinese survey sample was from state schools in the Beijing area using multistage strata and random cluster method. The detailed information of sampling procedure in China can be found elsewhere [19]. The response rates for schools and students in China were $91.5 \%$ and $98.1 \%$ respectively. The Finnish survey sample was gathered from state schools through Finland via cluster sampling with Probability Proportional to Size (PPS) of schools method and the response rates for schools and students in Finland were $86.9 \%$ and $88.2 \%$ respectively. The data analysed in this study consisted of 1685 Finnish and 2005 Chinese 15-year-old adolescents. Overall, the sample size, gender proportion and the mean age are very similar in the two countries (Table 1).

Table 1 Demographic characteristics of respondents by country

\begin{tabular}{|c|c|c|c|c|c|c|c|c|}
\hline & \multicolumn{4}{|c|}{ China } & \multicolumn{4}{|c|}{ Finland } \\
\hline & $\mathrm{n}$ & $\%$ & $\begin{array}{l}\text { Mean } \\
\text { Age }\end{array}$ & SD & $\mathrm{n}$ & $\%$ & $\begin{array}{l}\text { Mean } \\
\text { Age }\end{array}$ & $\mathrm{SD}$ \\
\hline Boys & 892 & 44.5 & 15.82 & 0.32 & 790 & 46.9 & 15.79 & 0.33 \\
\hline Girls & 1113 & 55.5 & 15.75 & 0.33 & 895 & 53.1 & 15.76 & 0.31 \\
\hline Total & 2005 & 100 & 15.78 & 0.32 & 1685 & 100 & 15.78 & 0.32 \\
\hline
\end{tabular}

Instrument and variables

The HBSC survey was based on completion of a self-report questionnaire during a normal school class. The students were instructed on how to fill in the questionnaire and also informed that only the researcher will read their answers. Student's participation in the survey was voluntary and anonymous. The questionnaire used in the 
Chinese survey was based on the English version of the questionnaire used in the Finnish HBSC Survey in 2006. The questionnaire was firstly translated from English to Chinese by two researchers independently and re-translated from Chinese to English by other professional translators to check for any discrepancies. The survey questionnaire has satisfactory test-retest reliability for the students in Beijing [20].

\section{Measurements of smoking}

Ever smoked

The issue of whether students had ever smoked was examined by asking the question 'have you ever smoked (at least one cigarette, cigar or a pipeful)?' The answers were: 'yes', or 'no'. Respondents who answered yes were defined as ever smoked.

Present status of smoking

The present status of smoking was assessed by asking the students 'how often do you smoke at present?' The response alternatives were: 'every day', 'every week, but not daily', 'less than once a week', 'I do not smoke'. A daily smoker was defined as those students reporting that they smoke every day. Regular smoker was defined as respondents who reported that they smoke at least once every week. All the other respondents were defined as current smoker except for those who answered that they do not smoke, and missing answers.

\section{Onset of smoking}

The initiation of smoking was examined by the item asking 'at what age did you smoke a cigarette (more than a puff) for the first time?' The answers were: 'never', '11 years old or less', '12 years old', ' 13 years old', '14 years old', '15 years old or older'. Early onset of smoking was defined as those respondents who reported first smoking at age 13 years or younger.

\section{Measurements of socioeconomic status}

\section{Family Affluence Scale}

The 'Family Affluence Scale' (FAS) has been used to examine and explain socioeconomic inequalities in the HBSC study for more than 10 years, and it has also been proven as a reliable and valid SES measure for adolescents in China [19]. The scale compromises four items: 1$)$ does your family own a car (0, 1, 2 or more)? 2) do you have your own room $(0,1)$ ? 3) how many times over the last year did you travel away somewhere on holiday with your family (0, 1, 2, 3 or more)? 4) how many computers does your family own $(0,1,2,3$ or more)? A composite FAS score was calculated by summing the responses of these four items ranging from 0 to 7 . The composite scores were subsequently recoded into high $(6,7)$, middle $(4,5)$ and low (0-3) family affluence groups.

Perceived Family Wealth

The item related to perceived family wealth (PFW) asked the adolescents 'what do you think of the financial situation of your family?' The answers were: 'my family is very well off financially', 'my family is quite well off financially', 'average', 'my fam- 
ily is not very well off financially', and 'my family is not at all well off financially'. Response options were categorized into high (very well off and quite well off), middle (average), and low (not very well off and not well off at all) perceived family wealth groups.

The Table 2 shows the distribution and correlation of FAS and PFW in China and Finland. Although the correlation between FAS and PFW was higher in China than the correlation of Finland, they were significantly correlated in both countries.

Table 2 Distribution and correlation of SES variables by country

\begin{tabular}{lllllll}
\hline & & \multicolumn{2}{c}{ China } & & \multicolumn{2}{c}{ Finland } \\
\cline { 3 - 4 } \cline { 6 - 7 } & & $\mathrm{n}$ & $\%$ & & $\mathrm{n}$ & $\%$ \\
\hline FAS & & & & & \\
& Low & 1025 & 51.1 & & 262 & 15.6 \\
& Middle & 649 & 32.4 & & 792 & 47.0 \\
& High & 331 & 16.5 & & 613 & 37.4 \\
& Total & 2005 & 100 & & 1685 & 100 \\
PFW & & & & & \\
& Low & 370 & 18.5 & & 72 & 4.3 \\
& Middle & 1017 & 50.7 & & 519 & 30.8 \\
& High & 618 & 30.8 & & 1094 & 64.9 \\
& Total & 2005 & 100 & & 1685 & 100 \\
FAS-PFW & $0.511^{* *}$ & & $0.241^{* *}$ \\
correlation & & & & \\
\hline
\end{tabular}

Note: ** Significance at 0.01 level.

Statistical analyses

Descriptive statistics were used to show the characteristics of the study sample and the distribution of independent and dependent variables. Pearson correlation was used to examine the relationship between FAS and PFW. Differences in percentage of smoking behaviour were compared by gender, FAS and PFW for China and Finland respectively using Pearson's Chi-square test. In addition, Pearson's Chi-square test was also used to compare the prevalence of smoking behaviour of total population by country. Binary logistic regression models were used to investigate the relationships between all variables of smoking behaviour and FAS and PFW for the two countries separately. In each model, gender was adjusted and the high FAS or PFW group served as the reference category. Odds ratios with $95 \%$ confidence intervals (CIs) were computed for other categories of independent variables. A $p$ value of < 0.05 or $95 \%$ CIs do not overlap were considered statistically significant. All analyses were done by using Analytics Software (PASW, formerly SPSS), version 18.0 (SPSS, Inc., Chicago, Illinois, US).

\section{Results}

The prevalence rates of smoking behaviour of Chinese and Finnish school-aged children are shown in Table 3. In general, for all five variables of smoking behaviour, the 
results indicated that the prevalence is much higher among Finnish schoolchildren than Chinese schoolchildren $(p<0.001)$, especially demonstrated by the percentage of Finnish daily smokers being nearly seven times as many as the Chinese. Nearly one fifth $(17.8 \%)$ of Chinese adolescents reported they had ever smoked and $11.6 \%$ of them smoked when they were 13 years or younger. This compares to the Finnish results which showed that more than half (59.3\%) of them have smoked, and 33.6\% of them were classified as an early smoker. Among Chinese respondents, gender difference was observed for all measures; boys exhibiting greater prevalence of smoking than girls $(p<0.001)$. In contrast, there were no differences of the percentage of ever smoked $(p<0.213)$, current smoker $(p<0.379)$ and regular smoker $(p<$ 0.337 ) between Finnish boys and girls, although more boys were daily smoker or early smoker than girls. The differences of daily smoker rate by FAS groups were observed in both countries with a different trend. In addition, the differences of ever smoked by PFW groups and early smoker by FAS categories were found in China but not in Finland.

Table 3 Prevalence of smoking by gender and SES group in China and Finland

\begin{tabular}{|c|c|c|c|c|c|c|c|c|c|c|c|c|c|c|c|}
\hline & \multicolumn{3}{|c|}{ Ever smoked } & \multicolumn{3}{|c|}{ Current smoker } & \multicolumn{3}{|c|}{ Regular smoker } & \multicolumn{3}{|c|}{ Daily smoker } & \multicolumn{3}{|c|}{ Early onset } \\
\hline & $\mathrm{n}$ & $\%$ & $p$ & $\mathrm{n}$ & $\%$ & $p$ & $\mathrm{n}$ & $\%$ & $p$ & $\mathrm{n}$ & $\%$ & $p$ & $\mathrm{n}$ & $\%$ & $p$ \\
\hline \multicolumn{16}{|l|}{ China } \\
\hline Total & 356 & 17.8 & & 112 & 5.5 & & 92 & 4.6 & & 49 & 2.4 & & 233 & 11.6 & \\
\hline Gender & & & $* * *$ & & & $* * *$ & & & $* * *$ & & & $* * *$ & & & ns \\
\hline Boy & 265 & 29.7 & & 102 & 11.5 & & 86 & 9.6 & & 49 & 5.5 & & 175 & 19.6 & \\
\hline Girl & 91 & 8.2 & & 10 & 0.9 & & 6 & 0.5 & & 0 & 0.0 & & 58 & 5.2 & \\
\hline FAS & & & ns & & & ns & & & ns & & & $* *$ & & & * \\
\hline Low & 197 & 19.4 & & 51 & 5.0 & & 41 & 4.0 & & 20 & 2.0 & & 132 & 13.0 & \\
\hline Middle & 97 & 14.9 & & 36 & 5.5 & & 30 & 4.6 & & 12 & 1.8 & & 59 & 9.1 & \\
\hline High & 60 & 18.1 & & 24 & 7.3 & & 20 & 6.0 & & 16 & 4.8 & & 41 & 12.4 & \\
\hline PFW & & & * & & & ns & & & ns & & & ns & & & ns \\
\hline Low & 82 & 22.2 & & 25 & 6.8 & & 22 & 6.0 & & 12 & 3.3 & & 53 & 14.7 & \\
\hline Middle & 179 & 17.6 & & 50 & 4.9 & & 39 & 3.8 & & 19 & 1.9 & & 116 & 11.6 & \\
\hline High & 93 & 15.2 & & 36 & 5.9 & & 31 & 5.1 & & 18 & 2.9 & & 62 & 10.3 & \\
\hline \multicolumn{16}{|l|}{ Finland } \\
\hline Total & 1000 & 59.3 & $\#$ & 499 & 29.5 & $\#$ & 369 & 21.9 & $\#$ & 280 & 16.6 & $\#$ & 586 & 33.6 & $\#$ \\
\hline Gender & & & ns & & & ns & & & ns & & & * & & & $* *$ \\
\hline Boy & 481 & 60.9 & & 242 & 30.7 & & 181 & 22.9 & & 148 & 18.7 & & 287 & 36.3 & \\
\hline Girl & 519 & 58.0 & & 257 & 28.7 & & 188 & 21.0 & & 132 & 14.7 & & 279 & 31.2 & \\
\hline FAS & & & ns & & & ns & & & * & & & $* *$ & & & $\mathrm{~ns}$ \\
\hline Low & 134 & 60.4 & & 67 & 30.2 & & 54 & 24.3 & & 48 & 21.6 & & 83 & 37.4 & \\
\hline Middle & 483 & 61.0 & & 248 & 31.4 & & 190 & 24.0 & & 144 & 18.2 & & 273 & 34.5 & \\
\hline High & 365 & 57.8 & & 175 & 27.8 & & 119 & 18.9 & & 82 & 13.0 & & 199 & 31.5 & \\
\hline PFW & & & ns & & & ns & & & $\mathrm{ns}$ & & & * & & & ns \\
\hline Low & 50 & 69.4 & & 25.0 & 34.7 & & 21 & 29.2 & & 20 & 27.8 & & 33 & 45.8 & \\
\hline Middle & 297 & 57.2 & & 139.0 & 26.8 & & 102 & 19.7 & & 80 & 15.4 & & 168 & 32.9 & \\
\hline High & 642 & 59.6 & & 328.0 & 30.5 & & 239 & 22.2 & & 175 & 16.2 & & 356 & 34.0 & \\
\hline
\end{tabular}


The logistic regression analyses of smoking and FAS were performed in both countries and the results can be seen from Table 4 . When the smoking behaviour of students from the high FAS group was used as a reference group, we found different relationships of daily smoker and FAS between China and Finland. For Chinese youth, students from the low $(\mathrm{OR}=0.49,95 \% \mathrm{CI}$ : $0.25-0.98)$ and the middle $(\mathrm{OR}=$ $0.43,95 \%$ CI: $0.20-0.92)$ FAS group are less likely to be a daily smoker. On the contrary, Finnish students from the low (OR $=1.84,95 \% \mathrm{CI}$ : 1.24-2.74) and the middle (OR $=1.51,95 \% \mathrm{CI}: 1.13-2.03$ ) FAS group are more likely to report being a daily smoker. Moreover, a difference was found for the regular smoker in Finland, in that students in the middle FAS group were 1.37 times more likely to be a regular smoker than those from the high FAS group.

Table 4 The associations between smoking and FAS and SSS by country with gender adjusted, odds ratios with $95 \%$ confidence interval

\begin{tabular}{|c|c|c|c|c|c|c|c|c|c|c|}
\hline & \multicolumn{2}{|c|}{$\begin{array}{l}\begin{array}{l}\text { Ever } \\
\text { smoked }\end{array} \\
\end{array}$} & \multicolumn{2}{|c|}{$\begin{array}{l}\text { Current } \\
\text { smoker }\end{array}$} & \multicolumn{2}{|c|}{$\begin{array}{l}\text { Regular } \\
\text { smoker }\end{array}$} & \multicolumn{2}{|c|}{$\begin{array}{l}\text { Daily } \\
\text { smoker }\end{array}$} & \multicolumn{2}{|c|}{$\begin{array}{l}\text { Early } \\
\text { onset }\end{array}$} \\
\hline & OR & $95 \% \mathrm{CI}$ & OR & $95 \% \mathrm{CI}$ & OR & $95 \% \mathrm{CI}$ & OR & $95 \% \mathrm{CI}$ & OR & $95 \% \mathrm{CI}$ \\
\hline \multicolumn{11}{|l|}{ China } \\
\hline \multicolumn{11}{|l|}{ FAS } \\
\hline Low & 1.30 & $0.93-1.82$ & 0.83 & $0.50-1.39$ & 0.81 & $0.46-1.42$ & 0.49 & $0.25-0.98$ & 1.23 & $0.84-1.81$ \\
\hline Middle & 0.88 & $0.61-1.26$ & 0.86 & $0.50-1.50$ & 0.87 & $0.48-1.58$ & 0.43 & $0.20-0.92$ & 0.76 & $0.49-1.17$ \\
\hline High & 1 & & 1 & & 1 & & 1 & & 1 & \\
\hline \multicolumn{11}{|l|}{ PFW } \\
\hline Low & 1.92 & $1.36-2.71$ & 1.42 & $0.82-2.45$ & 1.46 & $0.82-2.61$ & 1.38 & $0.65-2.94$ & 1.79 & $1.19-2.68$ \\
\hline Middle & 1.32 & $0.99-1.75$ & 0.92 & $0.59-1.44$ & 0.83 & $0.51-1.36$ & 0.71 & $0.36-1.37$ & 1.28 & $0.91-1.79$ \\
\hline High & 1 & & 1 & & 1 & & 1 & & 1 & \\
\hline \multicolumn{11}{|l|}{ Finland } \\
\hline \multicolumn{11}{|l|}{ FAS } \\
\hline Low & 1.11 & $0.81-1.52$ & 1.12 & $0.80-1.57$ & 1.38 & $0.96-1.99$ & 1.84 & $1.24-2.74$ & 1.32 & $0.96-1.82$ \\
\hline Middle & 1.15 & $0.93-1.42$ & 1.19 & $0.95-1.50$ & 1.37 & $1.06-1.77$ & 1.51 & $1.13-2.03$ & 1.18 & $0.94-1.48$ \\
\hline High & 1 & & 1 & & 1 & & 1 & & 1 & \\
\hline \multicolumn{11}{|l|}{ PFW } \\
\hline Low & 1.56 & $0.93-2.62$ & 1.22 & $0.74-2.02$ & 1.46 & $0.86-2.48$ & 2.06 & $1.19-3.54$ & 1.75 & $1.08-2.84$ \\
\hline Middle & 0.92 & $0.74-1.14$ & 0.84 & $0.67-1.07$ & 0.87 & $0.67-1.13$ & 0.98 & $0.73-1.31$ & 0.98 & $0.78-1.23$ \\
\hline High & 1 & & 1 & & 1 & & 1 & & 1 & \\
\hline
\end{tabular}

We further employed the logistic regression models to investigate the associations between smoking and PFW (Table 4). Unlike the previous results based on the FAS groups, significant differences were also found for early onset of smoking in both countries, and ever smoked in China. With the high PFW group as the reference, these results indicated that young people in both countries who perceive their family wealth as low are more likely to start smoking at 13 years old or younger (1.79 times for China and 1.75 times for Finland), and Chinese respondents who were from low PFW groups are 1.92 times more likely to have ever smoked. The significant differences between daily smoker and PFW groups, however, were only found in Finland, 
specifically that the students from low PFW are about two times $(\mathrm{OR}=2.06)$ more likely to smoke daily.

\section{Discussion}

The current study investigated the smoking prevalence among 15 year-old schoolchildren in China and Finland, and compared the socioeconomic inequalities in adolescents' smoking between two countries. The main findings of this study are that the socioeconomic inequalities in adolescents' smoking, based on certain indicators of smoking behaviour by objective (FAS) and subjective (PFW) measures of SES, were observed in both China and Finland, and the socioeconomic patterns of smoking are different between these two countries.

Various results have been reported from previous studies concerning the socioeconomic inequalities in adolescents' smoking. However, it should be noted that those studies were mainly from western countries. Our findings regarding Chinese adolescents indicated that young people from a less affluent family background are less likely to smoke daily, which is not consistent with the above-mentioned findings, but consistent with a recent Chinese study which reported that higher income relative to others is a risk factor for smoking among urban girls but not urban boys [21]. On the contrary, with regard to Finnish results, a different pattern was found, showing that students from low and middle affluence families are at higher risk of being daily smokers. This result is in contrast to the study done by Paavola and her colleagues in Finland [11], but is in line with a study which examined the socioeconomic differences in smoking among Finnish adolescents from 1977 to 2007, in which high rates of smoking were found in lower SES groups which persisted over time [22]. However, it is noteworthy to point out the inconsistent or consistent pattern of socioeconomic inequalities in adolescents' smoking revealed between present and previous studies should be viewed with caution due to the different measures of smoking and SES used, as well as different sampling strategies used in these studies which makes the results incomparable.

Smoking in adolescence is influenced by many factors. From the individual level, such as demographic factors (age, gender, ethnicity, SES), physiological differences (genetics, physiology reaction), and other risk behaviour, to the contextual lev$\mathrm{el}$, such as peer relationships, parents and families' smoking, environmental/culture context (tobacco advertising and media message, taxation, cost and policies) [23]. Thus, there are several plausible explanations for the different pattern of socioeconomic inequalities in daily smoking between Chinese and Finnish adolescents, as observed in our study. The first explanation is the adolescents' availability of resources to purchase tobacco products, since the amount of pocket money adolescents have has a strong relationship with current smoking [24]. There is a huge difference in national income and income inequality between China and Finland. China is a country with a rather high income inequality, and adolescents who are part of an affluent family usually have more pocket money and hence they have more possibility to buy cigarettes for smoking than those in less affluent families. However, with regard to Finnish adolescents, although the amount of pocket money of young people in different FAS groups may still vary, even those who live in rather less affluent families might have enough money to buy cigarettes due to the lower income ine- 
quality. Another possible explanation for the difference could be due to the different transition stages of smoking in China and Finland. It was reported that the prevalence of smoking has been falling since the 1970's in Nordic countries [25]. However, the smoking rates have not decreased simultaneously and equally in different socioeconomic groups. The rate of smoking among the higher social gradient has fallen more quickly, which leads to increasing inequalities of the smoking rate in different socioeconomic groups [26].

Interestingly, no significant difference was found for current smoker among the three FAS or PFW groups, and any associations between ever smoked and regular smoker, and SES were also rarely found, which demonstrated that the socioeconomic inequalities may be neglected if we did not include daily smoker as the indicator of smoking. Additionally, adolescents from the lower perceived family wealth group were more likely to be early onset smokers in both countries, which the pattern was not significant if FAS was used as a SES measures instead of PFW. The results suggest that a certain smoking measure may be sensitive to a certain measure of SES, which also provides the evidence that the inconsistent or even contradictionary patterns of socioeconomic inequalities in previous studies may possibly be due to employing different measures of smoking and SES. Thus, when further studies focusing on such topics are conducted, regardless of location, researchers should be aware of the possible effect of choice of measurement methods.

To our knowledge, this study is the first to examine and compare the socioeconomic inequalities in adolescent smoking between a European country and an Asian country. Research on socioeconomic inequalities in smoking between countries rather than within one country can provide evidence that socioeconomic status may not influence smoking independently of cultural and political contexts, and the variance presented in this study may reflect how different aspects SES can influence one certain health behaviour in different countries. On the one hand, from a theoretical point of view, findings from this study can broaden our knowledge in understanding socioeconomic inequalities of smoking in adolescence. On the other hand, from the practical point of view, the evidence-based findings from this study can provide valuable information for policy makers and health practitioners. In China, when planning and implementing interventions targeting the reduction of smoking of boys from more affluent families, and in Finland when deciding if policies should be put into place aimed at reducing the high rates of smoking of adolescents from lower SES groups in both genders.

Several methodological limitations in the present study should be addressed. First of all, this study did not include the mediating factors, such as family structure and school types, which may also influence the results. Second, the Chinese data used in this study were only sampled from the Beijing municipal area due to the aims of the survey and the limited resources. As a country with huge diversity such as China, the results should be interpreted with caution since the sample cannot represent the whole nation. Third, the two SES measures used in this study, FAS and PFW, are not evenly distributed in both countries, for instance, there are rather less cases of low PFW groups among Finnish samples. In spite of the fact that FAS has a very high response rate in the present study, and it has been proved to be a reliable and valid measure of family wealth between cultures [27] and countries [28], it is still 
a reflection of family material affluence which may not be absolutely consistent with the available resources that schoolchildren can use to buy cigarettes for smoking. This situation is also true for PFW. Moreover, self-reporting of smoking behaviour could also raise bias due to the social desirability of smoking among adolescents'.

\section{Conclusions}

In conclusion, the present study indicated different patterns of socioeconomic inequalities in daily smoking between Chinese and Finnish adolescents. Chinese 15year-old students from high affluence families are more likely to exhibit daily smoking than those from lower affluence families. In comparison, high rates of daily smoking were found in lower FAS groups in Finland. However, in both countries, early onset of smoking was more prevalent among students who perceived their family wealth as low. Considering the culture and income differences between China and Finland, specific policies and interventions for different target groups should be encouraged and designed for reducing the socioeconomic inequalities in adolescents' smoking. Further studies on socioeconomic inequalities in adolescent smoking should be conduct with caution, by selecting sensitive indicators measuring SES as well as smoking behaviour to understand this phenomenon comprehensively.

\section{Competing interests}

The authors declare that there is no potential conflict of interest with regards to this research.

\section{Authors' contributions}

YL (the first author) designed the study, lead the analysis and drafted the manuscript. MW, JT, YL, and KL participated in refining the study's design, involved in writing the manuscript and gave critical comments on it. JV helped to analyses the data and commented on the manuscript. All authors read and approved the final manuscript.

\section{Acknowledgements}

This work was supported by the China Institute of Sport Science (CISS) and the Research Centre for Health Promotion in the Department of Health Sciences at the University of Jyväskylä. The data collection in China was funded by the CISS and the data collection in Finland was funded by the Ministry of Social Affairs and Health of Finland. The first author was supported by the grants from the Juho Vainio Foundation and the University of Jyväskylä.The authors would like to thank Ms. Lanmin Xiao for helping to organize the school survey in Beijing and Mr. Zhouyang Zhang for helping to conduct the field work. The authors also would like to thank Mr. Michael Ormshaw for checking the language of the manuscript. 


\section{References}

1. U.S. Department of Health and Human Services: How tobacco smoke causes disease: The biology and behavioural basis for smoking-attributable disease: A report of the Surgeon General 2010. Atlanta, GA; 2010.

2. WHO Report on the Global Tobacco Epidemic 2008: The MPOWER Package. Geneva; 2008.

3. Mistry R, McCarthy W, de Vogli R, Crespi, CM, Wu Q, Patel M: Adolescent smoking risk increases with wider income gaps between rich and poor. Health Place 2011, 17:222-229.

4. Jha P, Peto R, Zatonski W, Boreham J, Jarvis MJ, Lopez AD: Social inequalities in male mortality and in male mortality from smoking: Indirect estimation from national death rates in England and Wales, Poland and North America. Lancet 2006, 368:367-370.

5. Mackenbach JP, Stirbu I, Roskam AJ, Schaap MM, Menvielle G, Leinsalu M, Kunst AE: European Union Working Group on Socioeconomic Inequalities in Health: socioeconomic Inequalities in Health in 22 European Countries. $N$ Engl J Med 2008, 358(23):2468-2481.

6. Crone MR, Reijneveld SA, Willemsen MC, van Leerdam FJM, Spruijt RD, Sing RAH: Prevention of smoking in adolescents with lower education: a school based intervention study. J Epidemiol Community Health 2003, 57(9):675-680.

7. Koivusilta LK, Rimpela AH, Kautiainen SM: Health inequality in adolescence: does stratification occur by familial social background, family affluence, or personal social position? BMC Public Health 2006, 6:110.

8. Piko BF, Fitzpatrick KM: Socioeconomic Status, Psychosocial Health and Health Behaviours among Hungarian Adolescents. Eur J Public Health 2007, 17(4):353-360.

9. Gecková A Stewart R, van Dijk JP, Orosová O, Groothoff JW, Post D: Influence of socio-economic status, parents and peers on smoking behaviour of adolescents. Eur Addict Res 2005, 11:204-209.

10. Schnohr CW, Kreiner S, Rasmussen M, Due P, Diderichsen F: School-related mediators in social inequalities in smoking: a comparative cross-sectional study of 20399 adolescents. Int J Equity Health 2009, 8:17.

11. Paavola M, Vartiainen E, Haukkala A: Smoking from adolescence to adulthood. The effects of parental and own socioeconomic status. Eur J Public Health 2004, 14:417-421.

12. Simetin IP, Kuzman M, Franelic IP, Pristas I, Benjak T, Dezeljin JDD: Inequalities in Croatian pupils' unhealthy behaviours and health outcomes: role of school, peers and family affluence. Eur J Public Health 2010, 21(1):122-128.

13. Richter M, Leppin A: Trends in socio-economic differences in tobacco smoking among German schoolchildren, 1994-2002. Eur J Public Health 2007, 17(6):565-571.

14. Currie C, Nic Gabhainn S, Godeau E, Roberts C, Smith R, Currie D, Pickett W, Richter M, Morgan A, Barnekow V: Inequalities in young people's health: HBSC international report from the 2005/06 Survey. Copenhagen; WHO Regional Office for Europe, 2008. 
15. Zhang H, Cai B: The impact of tobacco on lung health in China. Respirology 2003, 8:17-21.

16. Lopez AD, Collishaw NE, Piha T: A descriptive model of the cigarette epidemic in developed countries. Tob Control 1994, 3:242-247.

17. Currie C, Zanotti C, Morgan A, Currie D, de Looze M, Roberts C, Samdal O, Smith R, Barnekow V: Social determinants of health and well-being among young people. Health Behaviour in School-aged Children (HBSC) study: international report from the 2009/2010 survey. Copenhagen; WHO Regional Office for Europe, 2012.

18. Currie C, Samdal O, Boyce W, Smith B: Health Behaviour in School-aged Children: a WHO Cross-National Study (HBSC), Research Protocol for the 2001/2002 Survey. Edinburgh; Child and Adolescent Health Research Unit (CAHRU), University of Edinburgh, 2001.

19. Liu Y, Wang M, Villberg J, Torsheim T, Tynjälä J, Lv Y, Kannas L: Reliability and Validity of Family Affluence Scale (FAS II) among Adolescents in Beijing, China. Child Indic Res 2012, 5(2):235-251.

20. Liu Y, Wang M, Tynjälä J, Lv Y, Villberg J, Zhang Z, Kannas L: Test-retest reliability of selected items of Health Behaviour in School-aged Children (HBSC) survey questionnaire in Beijing, China. BMC Med Res Methodol 2010, 10:73.

21. Sun P, Unger JB, Palmer P, Ma H, Xie B, Sussman S, Johnson CA: Relative income inequality and selected health outcomes in urban Chinese youth. Soc Sci Med 2012, 74:84-91.

22. Doku D, Koivusilta L, Rainio S, Rimpelä A: Socioeconomic differences in smoking among Finnish adolescents from 1977 to 2007. J Adolescent Health 2010, 47(5):479-487.

23. Turner L, Mermelstein R, Flay B: Individual and contextual influences on adolescent smoking. Ann NY Acad Sci 2004, 1024:175-197.

24. West P, Sweeting H, Young R: Smoking in Scottish youth: personal income, parental social class and the cost of smoking. Tob Control 2007, 16(5):329-335.

25. Cavelaars AE, Kunst AE, Geurts JJ, Crialesi R, Grötvedt L, Helmert U, Lahelma E, Lundberg O, Matheson J, Mielck A, Rasmussen NK, Regidor E, do RosárioGiraldes M, Spuhler T, Mackenbach JP: Educational differences in smoking: international comparison. Brit Med J 2000, 320(7242):1102-1107.

26. Giskes K, Kunst AE, Benach J, Borrell C, Costa G, Dahl E, Dalstra JA, Federico B, Helmert U, Judge K, Lahelma E, Moussa K, Ostergren PO, Platt S, Prattala R, Rasmussen NK, Mackenbach JP: Trends in smoking behaviour between 1985 and 2000 in nine European countries by education. J Epidemiol Community Health 2005, 59(5):395-401.

27. Brown RA, Adler NE, Worthman CM, Copeland WE, Costello EJ, Angold A: Cultural and community determinants of subjective social status among Cherokee and White youth. Ethnic Health 2008, 13(4):289-303.

28. Schnohr CW, Kreiner S, Due P, Currie C, Boyce W, Diderichsen F: Differential item functioning of a family affluence scale: validation study on data from HBSC 2001/02. Soc Indic Res 2008;89(1):79-95. 